Oceanography and Marine Biology: An Annual Review, 2005, 43, 279-418

(C) R. N. Gibson, R. J. A. Atkinson, and J. D. M. Gordon, Editors

Taylor \& Francis

\title{
THE ECOLOGY OF RAFTING IN THE MARINE ENVIRONMENT. II. THE RAFTING ORGANISMS AND COMMUNITY
}

\author{
MARTIN THIEL ${ }^{1,2^{*}} \&$ LARS GUTOW ${ }^{3}$ \\ ${ }^{1}$ Facultad Ciencias del Mar, Universidad Católica del Norte, Larrondo 1281, Coquimbo, Chile \\ ${ }^{2}$ Centro de Estudios Avanzados en Zonas Áridas (CEAZA), Coquimbo, Chile \\ *E-mail: thiel@ucn.cl; Fax: ++ 5651209812 \\ ${ }^{3}$ Alfred Wegener Institute for Polar and Marine Research, Biologische Anstalt Helgoland, \\ Box 180, 27483 Helgoland, Germany \\ E-mail: lgutow@awi-bremerhaven.de \\ * author for correspondence
}

\begin{abstract}
Rafting of marine and terrestrial organisms has been reported from a variety of substrata and from all major oceans of the world. Herein we present information on common rafting organisms and on ecological interactions during rafting voyages. An extensive literature review revealed a total of 1205 species, for which rafting was confirmed or inferred based on distributional or genetic evidence. Rafting organisms comprised cyanobacteria, algae, protists, invertebrates from most marine but also terrestrial phyla, and even a few terrestrial vertebrates. Marine hydrozoans, bryozoans, crustaceans and gastropods were the most common taxa that had been observed rafting. All major feeding types were represented among rafters, being dominated by grazing/boring and suspension-feeding organisms, which occurred on all floating substrata. Besides these principal trophic groups, predators/scavengers and detritus feeders were also reported. Motility of rafting organisms was highest on macroalgae and lowest on abiotic substrata such as plastics and volcanic pumice. Important trends were revealed for the reproductive biology of rafting organisms. A high proportion of clonal organisms (Cnidaria and Bryozoa) featured asexual reproduction, often in combination with sexual reproduction. Almost all rafting organisms have internal fertilisation, which may be due to the fact that gamete concentrations in the rafting environment are too low for successful fertilisation of external fertilisers. Following fertilisation, many rafting organisms incubate their offspring in/on their body or deposit embryos in egg masses on rafts. Local recruitment, where offspring settle in the immediate vicinity of parents, is considered an important advantage for establishing persistent local populations on a raft, or in new habitats. Some organisms are obligate rafters, spending their entire life cycle on a raft, but the large majority of reported rafters are considered facultative rafters. These organisms typically live in benthic (or terrestrial) habitats, but may become dispersed while being confined to a floating item. Substratum characteristics (complexity, surface, size) have important effects on the composition of the rafting community. While at sea, ecological interactions (facilitation, competition, predation) contribute to the community succession on rafts. Organisms capable to compete for and exploit resources on a raft (space and food) will be able to persist throughout community succession. The duration of rafting voyages is closely related to rafting distances, which may cover various geographical scales. In chronological order, three features of an organism gain in importance during rafting, these being ability to (1) hold onto floating items, (2) establish and compete successfully and (3) develop persistent local
\end{abstract}




\section{MARTIN THIEL \& LARS GUTOW}

populations during a long voyage. Small organisms that do not feed on their floating substratum and, with asexual reproduction or direct development, combine all these features appear to be most suited for long-distance dispersal on rafts and successful colonisation after reaching new habitats. All available evidence suggests that rafting is an important process for the population dynamics of many organisms and that it also has had and continues to have a strong influence on coastal biodiversity.

\section{Introduction}

... the agency of the Kafirkuils River in bringing down drift of the Riversdale coast at Still Bay after the great flood of November 1928 may be cited. Not only fruits, seeds, and other smaller vegetal matter were so transported, but railway construction plant, boats, ostriches, sheep, burrowing snakes (Typhlops), puff-adders and other Ophidia, lizards, tree-mice, scorpions, species of Coleoptera, and on previous occasions even a baboon or two.

\section{Muir (1937) reporting on drift material from beaches of South Africa}

Anecdotal reports such as this one by Muir (1937) or those of other authors (e.g., Guppy 1917, King 1962, Carlquist 1965, Van Duzer 2004) provide testimony of the diverse kinds of floating items that reach the oceans. Further, these reports give hints that many different organisms utilise these items as floating devices in order to escape from drowning. These observations of travellers on floating items also provoke many questions, the two most relevant ones being: where do these organisms come from, and where might they go? While these questions are simple and straightforward, finding the answers is not. In fact, biologists in the past have struggled to infer possible answers and in most cases evidence has remained circumstantial.

Organisms travelling on floating items over the sea surface may be transported to areas which they might not have reached otherwise. This process, termed rafting, can have important consequences if travellers, upon reaching new habitats, are capable of establishing new populations. Rafting is of particular importance for those organisms that are not capable of autonomous dispersal in or across the ocean. This is, for example, the case of many coastal organisms without pelagic dispersal stages. Also most terrestrial organisms are unable to travel over the sea without the aid of a transport vehicle.

In spite of a limited capacity for autonomous dispersal, many organisms have wide geographic distributions, even across wide oceanic barriers. This has led biologists to infer that disjunct distribution patterns could be the result of rafting on floating items. Distributional evidence has been reported for a wide diversity of organisms ranging from hydrozoans (Cornelius 1992a), small polychaetes (Knight-Jones \& Knight-Jones 1984), molluscs (Ó Foighil et al. 1999, Castilla \& Guiñez 2000), echinoderms (Mortensen 1933, Fell 1962 cited in Fell 1967) and crustaceans (Svavarsson 1982, Peck 1994), to terrestrial insects (Abe 1984, Niedbala 1998), reptiles (Raxworthy et al. 2002) and mammals (Hafner et al. 2001). In some of these cases, evidence for rafting as possible dispersal mechanism is better than in others. For example, in the case of the polychaete or mollusc species for which rafting was inferred, these live on/in macroalgae with a high floating potential. However, in other cases, the respective species with disjunct distributions have never been observed rafting and this process has only been suggested due to a lack of alternative explanations. Many organisms have also been observed in flagrante, i.e., while rafting on the high seas. In particular when coastal or terrestrial organisms were found on floating items far from the next shore, authors have suggested that these could potentially travel far distances on their rafts. Polychaetes (Arnaud et al. 1976, Averincev 1980), molluscs (Helmuth et al. 1994), echinoderms 


\section{RAFTING OF BENTHIC MARINE ORGANISMS}

(Hobday 2000a) and crustaceans (Ólafsson et al. 2001, Gutow \& Franke 2003) are frequently reported from floating items in the open ocean, often at far distances from the nearest coast.

Not all organisms are equally adapted to rafting. One of the most important preconditions is that a rafter needs to hold on to the substratum, and not all organisms can cling efficiently to floating items. Edgar \& Burton (2000) reported that many epifaunal organisms were rapidly lost from floating macroalgae. Feeding conditions on a floating item may also be very different from those in the natural habitats of most facultative rafters. Some organisms may even be capable of enduring a long journey on a floating item. During such a journey rafting organisms are also exposed to a variety of interactions with their substratum, fellow rafters and the water body in which they are travelling. Floating items provide attachment substratum and in many cases also food for rafters. In a rafting community one can expect similar ecological interactions as in benthic habitats (competition, facilitation, predation) and these may have profound effects on species succession during a long journey. In addition, oceanic conditions will affect rafters in various ways, e.g., in form of abiotic (wave action, temperature and salinity) and biotic (nutrients, food, predators) factors. Floating items travel at the sea surface, where environmental conditions might be considered as extreme for many species. At the water surface both inorganic and organic chemical compounds accumulate and also solar radiation is substantially higher than in the water column immediately below (Zaitsev 1970, Cheng 1975). Organisms on a raft are probably exposed to greater temperature changes and turbulence than encountered in their benthic habitat to which they are adapted (Holmquist 1994). All these interactions will have an influence on the survival of rafters during their journey and some space will be dedicated to these interactions in our review.

Rafting will only be a significant ecological and evolutionary process if rafters are capable of establishing new populations after reaching new coastal habitats. Whether new habitats can indeed be successfully colonised depends first on the general suitability of the habitat and second on the capacity of an arriving organism to reproduce or proliferate in a new habitat. The low proportion of potential rafting species coming from sandy habitats (Grantham et al. 2003) could suggest that organisms adapted to live in sandy bottoms are not well suited to survive on a raft and vice versa. The likelihood of successful colonisation may depend on the similarity between the original habitat of a rafting organism and the habitat in which it arrives after a voyage on a floating item. If a habitat is suitable, an arriving organism may be able to survive but in order to establish populations, successful reproduction or proliferation is required.

One of the most relevant issues in the context of rafting is its importance for local biodiversity and biogeography. The availability of floating items in an area determines the frequency of rafting opportunities and the connectivity between habitats. In many species without or with short-lived pelagic dispersal stages, rafting represents the main mechanism of gene flow within and among local populations. It is probable that the population dynamics of many coastal marine organisms with direct development are strongly influenced by rafting. This has been elegantly demonstrated by Worcester (1994) for a subpopulation of the compound ascidian Botrylloides sp.; even over relatively short distances (100s of $\mathrm{m}$ ) rafting of adult individuals on seagrass blades may be of similar or even higher importance than dispersal via pelagic larval stages (Figure 1). Thus, rafting is an important process in local population dynamics, yet estimation of dispersal distances and gene flow in species' populations that depend on rafting is scarce. This is surprising since (1) many coastal organisms have no pelagic dispersal stages and (2) information on exchange within and between local populations is crucial in the context of marine conservation. Design of marine protected areas (sizes and distances) critically depends on reasonable estimates of dispersal distances of organisms. For species with pelagic larvae, estimates typically are based on duration of the pelagic stage and current regime (Palumbi 2003, Shanks et al. 2003, Siegel et al. 2003) but since other studies have shown that many species from coastal habitats have no pelagic larval stages 


\section{MARTIN THIEL \& LARS GUTOW}

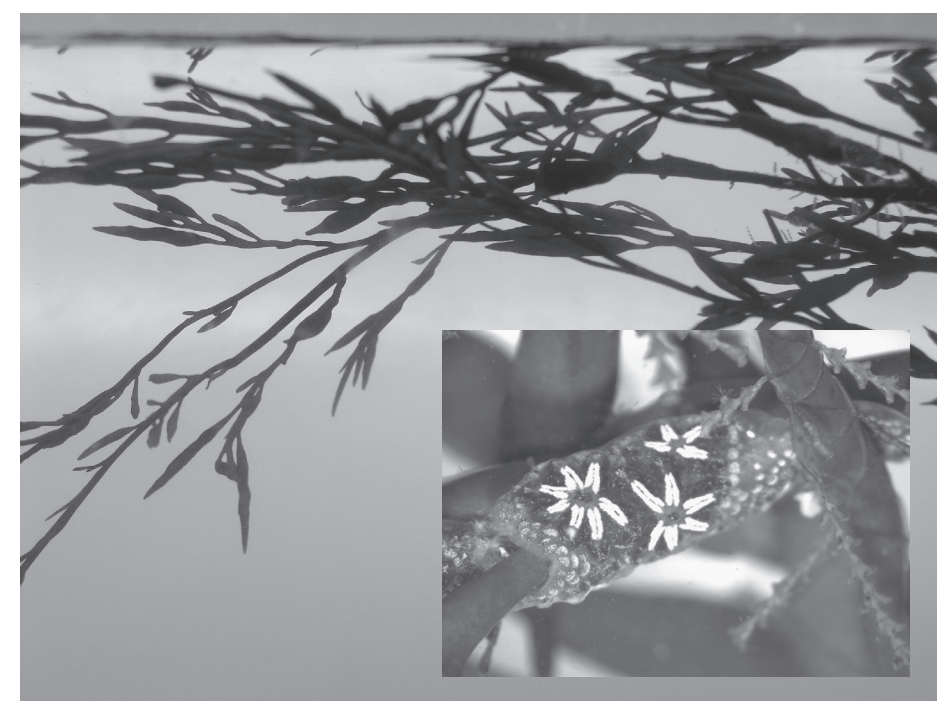

Figure 1 Common rafting organisms Botryllus schlosseri and some hydrozoans on Halidrys siliquosa cast ashore on the island of Helgoland during a storm in September 2004.

and a high likelihood of rafting (Grantham et al. 2003), it appears essential to incorporate estimates for rafting dispersal of these species in future studies (see also Kinlan \& Gaines 2003).

Recently, rafting has also received increasing attention as a potential mechanism for longdistance (>1000 km) transport of coastal organisms (Jokiel 1984, Castilla \& Guiñez 2000, Barnes \& Fraser 2003). In particular the vast amounts of plastic items floating in the world's oceans have caused concerns since these remain positively buoyant for long time periods and consequently may travel long distances (Winston et al. 1997, Aliani \& Molcard 2003). During recent decades the frequency of invasions by non-indigenous species in coastal marine habitats has increased (Ruiz et al. 2000), and while most of these are due to human-mediated transport (aquaculture or shipping activities, see Carlton 2003), in some cases invaders may have arrived on floating substrata.

These considerations already indicate that rafting as a process in the marine environment raises a variety of important questions. Clearly this process is of interest to biologists primarily due to the implications it has for dispersal of marine (and terrestrial) organisms. The probability that rafting organisms return to suitable habitats depends (1) on current directions and longevity of floating items and (2) on the capability of the rafting organism to survive the journey. While in a previous review (Thiel \& Gutow 2004) we explored the first point (abundance and characteristic of floating substrata), herein we will focus primarily on the second point (survival capacity of rafting organisms), and also discuss main processes during and at the end of a rafting journey.

For the present review we surveyed hundreds of original sources. The publications, which we examined for rafting organisms, probably can be best split into two categories - on the one hand those studies that concentrate on non-rafting topics (biogeography, phylogeny, genetic population structure, ecology) but infer that observed patterns (e.g., geographic species distribution) are influenced by rafting, and on the other hand those that are directly concerned with the process of rafting. Publications from the first category typically use the first line of evidence (distributional evidence), while those from the second category commonly examined the organisms on floating items (rafting evidence). In papers from the first category, evidence for rafting usually is circumstantial. We present a citation from Knight-Jones \& Knight-Jones (1984), who studied the biogeography of 


\section{RAFTING OF BENTHIC MARINE ORGANISMS}

spirorbid polychaetes, as a typical example for this: “... the widely distributed species ... is the one which is found most abundantly on Macrocystis pyrifera, a large alga with buoyant floats and a circumpolar distribution in the roaring forties". Often these interpretations do not take more than one sentence in the discussion of a publication. In the present review, we have incorporated many of these reports in which authors, based on all available evidence, suggest that rafting plays an important role in the biology of a species. While we believe that we have incorporated most of the relevant papers from this first category (distributional evidence), we cannot claim completeness. For studies from the second category (rafting evidence), i.e., those reporting on rafters on floating items, we are confident that we have considered the majority of published reports.

From the literature available to us we have extracted information on rafting species, their feeding and reproductive biology, geographic distribution and floating substrata on which they have been found. In many cases, authors provided this information in their publications but we occasionally consulted secondary literature to obtain information on the biology of a species. Herein we focus primarily on facultative rafters, i.e., coastal and terrestrial organisms that do not commonly live on floating items but potentially rely on them as a dispersal vehicle. Occasionally, we also include observations on obligate rafters and on pleuston organisms since these insights can help elucidate what characterises a good rafter. Similarly, we discuss some information on fishes associated with floating items but since most of these fish species are capable of autonomous dispersal we primarily deal with their ecological role in rafting communities. For complete coverage of those topics we refer readers to recent reviews (e.g., Castro et al. 2002, Andersen \& Cheng 2004).

The principal questions addressed herein are concerned with the organisms found rafting, their general biology, the ecology and succession of the rafting community, dispersal ecology of rafting biota and colonisation of new habitats. The general question we pursue herein is, what makes a good rafter? In order to answer this question we have planted several specific questions (Figure 2): Which taxa have been found or inferred to raft on floating items? Which biological traits are most common among rafting organisms? What is the outcome of successional changes during long

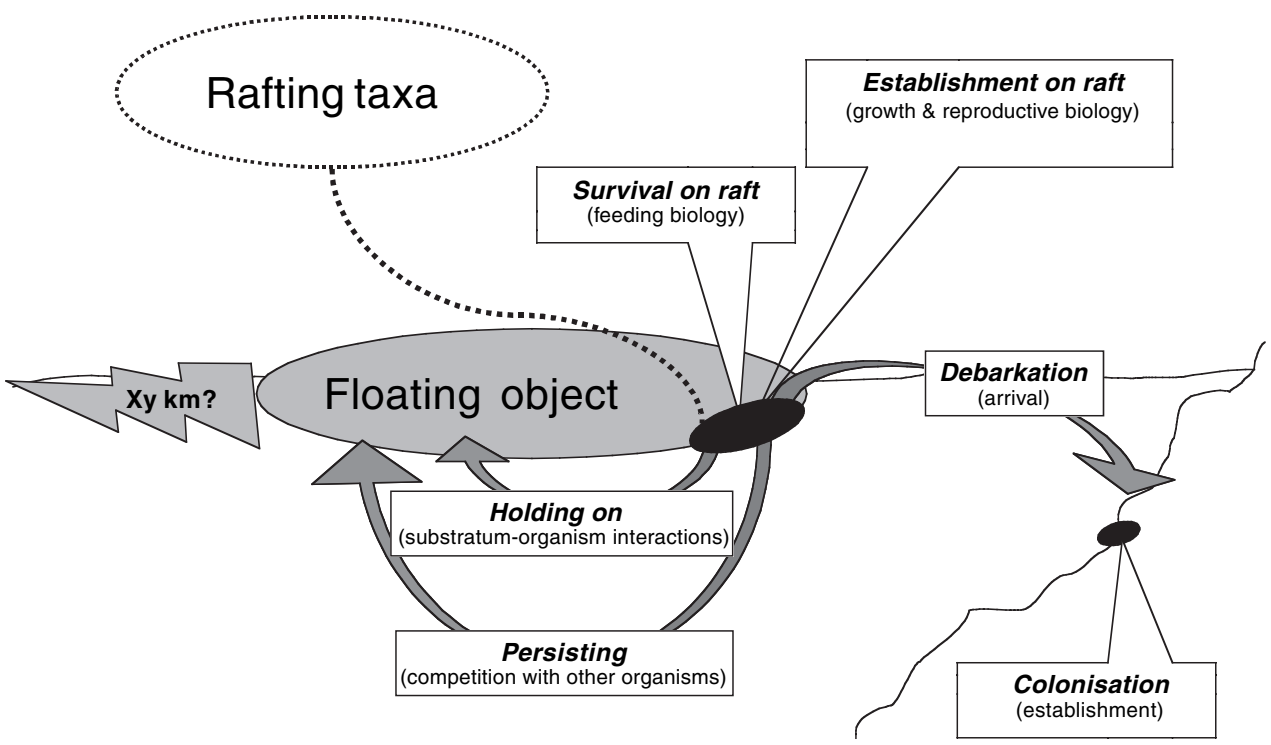

Figure 2 Schematic overview of some important questions concerning the dispersal potential of rafting organisms on floating items. 


\section{MARTIN THIEL \& LARS GUTOW}

journeys? What are the distances over which organisms may become dispersed? How does colonisation of new habitats proceed? Answers to these questions permit a better understanding of the evolution of coastal marine and terrestrial organisms. Knowing the degree of exchange via rafting within and between local populations is essential for estimates of connectivity between habitats. Rafting has been in the past (e.g., Scheltema 1977, Ingólfsson 1992) and continues to be an important dispersal mechanism in the world's oceans (Barnes 2002).

Finally we wish to emphasise that there exist many other mechanisms by which organisms (without or with limited pelagic stages) may become dispersed across wide oceanic distances (see, e.g., Ruiz et al. 2000, Domaneschi et al. 2002, Winkworth et al. 2002). For many species, rafting will be of no or only of minor importance. However, we believe that rafting is important on many different spatial and temporal scales, and evidence for this has been mounting during the past decades, in particular since the advent of molecular tools (e.g., Wares 2001, Wares \& Cunningham 2001, Sponer \& Roy 2002). Information on rafting in the marine environment is scattered over a wide area of scientific fields (e.g., geology, herpetology, terrestrial biogeography), sometimes making it difficult to evaluate this process. Our own background is marine biology and we cannot pretend to cover all these fields exhaustively. Yet, we hope that this review will be a useful synopsis in order to identify organisms for which rafting is (or has been) important. Based on the results of the present revision we formulate hypotheses and questions about possible consequences of rafting dispersal, which will be explored in a future contribution.

\section{Rafting organisms}

\section{Taxa and species}

Many different organisms have been found on floating substrata, among them unicellular organisms (Masó et al. 2003) and large vertebrates (Censky et al. 1998), as well as fully marine (Hobday 2000a) or terrestrial species (Heatwole \& Levins 1972). Rafting species can be facultative (e.g., Helmuth et al. 1994) or obligate (e.g., Gutow \& Franke 2003) inhabitants of floating substrata. Species may be highly selective with respect to the floating substrata they colonise or they may settle on any floating item available. For example, stalked barnacles (Lepadidae) have been reported from a wide diversity of different substrata including plastics (Carpenter \& Smith 1972, Aliani \& Molcard 2003), pumice (Coombs \& Landis 1966), wood (Nilsson-Cantell 1930), macroalgae (Arnaud 1973, Hobday 2000a) and even tar balls (Horn et al. 1970, Minchin 1996). In contrast, other species have only been reported from specific substrata, such as, e.g., the gastropod Littorina saxatilis, which exclusively rafts on floating macroalgae (Johannesson 1988, Ingólfsson 1995).

Herein we gathered information on a total of 1205 species, which have been either observed rafting or for which rafting had been inferred based on other evidence (distributional pattern, found on stranded items, genetic population structure). Completeness of this list is not claimed, yet we believe that it provides a representative overview of the most common rafting species and illustrates the general trends. Species from the phyla Cnidaria (Hydrozoa), Crustacea (Amphipoda) and Ectoprocta (= Bryozoa) were most commonly reported as rafters (Table 1). A total of 959 species are considered as facultative rafters, 41 species as obligate rafters and for 205 species information was insufficient to identify their status (at least four of this last group of species are capable of autonomous floating).

In this review we will present a taxonomic account of species found on floating substrata and whenever possible we will briefly discuss the propensity/capability of the respective taxa to settle and survive on floating substrata. Herein we primarily focus on organisms that depend on floating items to travel along the surface of the sea, i.e., organisms with negative buoyancy, which would otherwise sink to the sea floor. 


\section{RAFTING OF BENTHIC MARINE ORGANISMS}

Table 1 Number of species from the respective taxa that have been reported or inferred as rafting. Specific references for particular taxa are presented in Tables 2-14

\begin{tabular}{|c|c|c|c|c|}
\hline Taxon & $\begin{array}{l}\text { No. of } \\
\text { obligate rafters }\end{array}$ & $\begin{array}{l}\text { No. of } \\
\text { facultative rafters }\end{array}$ & $\begin{array}{l}\text { Insufficient } \\
\text { information }\end{array}$ & $\begin{array}{l}\text { Sum of } \\
\text { rafting species }\end{array}$ \\
\hline Cyanobacteria & 2 & 5 & 4 & 11 \\
\hline Fungi & 1 & 31 & 0 & 32 \\
\hline Chrysophyta & 0 & 3 & 11 & 14 \\
\hline Dinophyta & 0 & 15 & 0 & 15 \\
\hline Chlorophyta & 0 & 13 & 0 & 13 \\
\hline Phaeophyta & 0 & 19 & 0 & 19 \\
\hline Rhodophyta & 0 & 41 & 6 & 47 \\
\hline Bryophyta & 0 & 1 & 0 & 1 \\
\hline Magnoliopsida (vascular plants) & 0 & 16 & 22 & 38 \\
\hline Sarcomastigophora & 0 & 0 & 5 & 5 \\
\hline Ciliophora & 0 & 2 & 0 & 2 \\
\hline Porifera & 0 & 2 & 1 & 3 \\
\hline \multicolumn{5}{|l|}{ Cnidaria } \\
\hline Hydrozoa & 2 & 55 & 45 & 102 \\
\hline Anthozoa & 0 & 23 & 5 & 28 \\
\hline Scyphozoa & 0 & 1 & 0 & 1 \\
\hline \multicolumn{5}{|l|}{ Platyhelminthes } \\
\hline Turbellaria & 1 & 4 & 6 & 11 \\
\hline Nemertea & 0 & 2 & 0 & 2 \\
\hline Nematoda & 0 & 10 & 2 & 12 \\
\hline \multicolumn{5}{|l|}{ Annelida } \\
\hline Polychaeta & 5 & 62 & 12 & 79 \\
\hline Oligochaeta & 0 & 1 & 1 & 2 \\
\hline \multicolumn{5}{|l|}{ Arthropoda } \\
\hline Chelicerata (non-marine) & 0 & 12 & 0 & 12 \\
\hline Chelicerata (marine) & 0 & 4 & 3 & 7 \\
\hline Insecta & 0 & 26 & 0 & 26 \\
\hline Branchiopoda & 0 & 3 & 0 & 3 \\
\hline Cirripedia & 13 & 4 & 5 & 22 \\
\hline Copepoda (Cyclopoida) & 0 & 3 & 3 & 6 \\
\hline Copepoda (Calanoida) & 0 & 8 & 0 & 8 \\
\hline Copepoda (Harpacticoida) & 2 & 69 & 1 & 72 \\
\hline Ostracoda & 0 & 4 & 0 & 4 \\
\hline Leptostraca & 0 & 2 & 0 & 2 \\
\hline Euphausiacea & 0 & 2 & 0 & 2 \\
\hline Decapoda (Anomura) & 0 & 5 & 0 & 5 \\
\hline Decapoda (Brachyura) & 6 & 42 & 12 & 60 \\
\hline Decapoda (Caridea) & 0 & 19 & 4 & 23 \\
\hline Decapoda (Penaeoidea) & 0 & 5 & 1 & 6 \\
\hline Peracarida (Amphipoda) & 1 & 92 & 15 & 108 \\
\hline Peracarida (Isopoda) & 1 & 32 & 5 & 38 \\
\hline Peracarida (Tanaidacea) & 0 & 6 & 0 & 6 \\
\hline Tardigrada & 0 & 0 & 1 & 1 \\
\hline \multicolumn{5}{|l|}{ Mollusca } \\
\hline Polyplacophora & 0 & 3 & 0 & 3 \\
\hline Gastropoda (Archaeogastropoda) & 1 & 13 & 0 & 14 \\
\hline
\end{tabular}




\section{MARTIN THIEL \& LARS GUTOW}

Table 1 (continued) Number of species from the respective taxa that have been reported or inferred as rafting. Specific references for particular taxa are presented in Tables $2-14$

\begin{tabular}{|c|c|c|c|c|}
\hline Taxon & $\begin{array}{l}\text { No. of } \\
\text { obligate rafters }\end{array}$ & $\begin{array}{l}\text { No. of } \\
\text { facultative rafters }\end{array}$ & $\begin{array}{l}\text { Insufficient } \\
\text { information }\end{array}$ & $\begin{array}{l}\text { Sum of } \\
\text { rafting species }\end{array}$ \\
\hline Gastropoda (Mesogastropoda) & 2 & 36 & 1 & 39 \\
\hline Gastropoda (Neogastropoda) & 0 & 19 & 0 & 19 \\
\hline Gastropoda (Opisthobranchia) & 3 & 29 & 12 & 44 \\
\hline Gastropoda (Pulmonata) & 0 & 5 & 0 & 5 \\
\hline Bivalvia & 0 & 48 & 3 & 51 \\
\hline Cephalopoda & 0 & 8 & 3 & 11 \\
\hline \multicolumn{5}{|l|}{ Echinodermata } \\
\hline Crinoidea & 0 & 1 & 0 & 1 \\
\hline Asteroidea & 0 & 12 & 3 & 15 \\
\hline Echinoidea & 0 & 7 & 0 & 7 \\
\hline Holothuroidea & 0 & 6 & 0 & 6 \\
\hline Ophiuroidea & 0 & 9 & 2 & 11 \\
\hline Ectoprocta (Bryozoa) & 1 & 84 & 11 & 96 \\
\hline Chaetognatha & 0 & 2 & 0 & 2 \\
\hline \multicolumn{5}{|l|}{ Chordata } \\
\hline Tunicata & 0 & 11 & 0 & 11 \\
\hline Amphibia & 0 & 3 & 0 & 3 \\
\hline Reptilia & 0 & 17 & 0 & 17 \\
\hline Mammalia & 0 & 2 & 0 & 2 \\
\hline Total & 41 & 959 & 205 & 1205 \\
\hline
\end{tabular}

\section{Cyanobacteria}

Most reports on rafting cyanobacteria are from floating macroalgae Sargassum in the Sargasso Sea (Table 2). The heterocyst-bearing blue-green alga Dichothrix fucicola is a common epiphyte on floating Sargassum (Carpenter 1972). The standing crop of Dichothrix fucicola in the North Atlantic varies from about $1.5 \mathrm{mg} \mathrm{g}^{-1}$ Sargassum in the SW Sargasso Sea to about $70 \mathrm{mg} \mathrm{g}^{-1}$ Sargassum on the U.S. shelf off New Jersey and Delaware. These cyanobacteria may contribute substantially to primary production of the floating macroalgae (Carpenter \& Cox 1974). Furthermore, as the dominant epiphyte on floating $S$. natans, Dichothrix fucicola contributes significantly to the overall epiphyte respiration of the Sargassum community (Smith et al. 1973). Smith et al. (1973) refer to an unpublished manuscript by Carpenter when they mention nitrogen fixation as another important physiological function of this blue-green alga in the Sargasso Sea. Calothrix crustacea was also commonly found on Sargassum natans from Bermuda by Ryland (1974). Tar lumps covered with a grayish film were found to consume oxygen, which was attributed to the associated microorganisms (Butler 1975). Carpenter (1972) isolated rod-shaped gram-negative bacteria from the surface of polystyrene spherules. When fixed in formalin the oxygen uptake dropped to essentially zero. Microbial respiration comprises over 50\% of the respiration of the total Sargassum community in the Sargasso Sea and the adjacent continental Slope Water (Smith et al. 1973). The physiological significance of microbes in this open-ocean environment was attributed to the extensive surface area provided by the floating Sargassum plants. We consider it likely that cyanobacteria frequently occur on abiotic floating items where they may represent important initial colonisers in the biofilm assemblage. Floating macroalgae may actively suppress colonisation by cyanobacteria and other 


\section{RAFTING OF BENTHIC MARINE ORGANISMS}

Table 2 Cyanobacteria, Fungi, Algae and Vascular plants reported or inferred as rafting

\begin{tabular}{|c|c|c|c|c|}
\hline Species & Region & $\begin{array}{l}\text { Rafting } \\
\text { inference }\end{array}$ & $\begin{array}{l}\text { Floating } \\
\text { substratum }\end{array}$ & Reference \\
\hline \multicolumn{5}{|l|}{ Cyanobacteria } \\
\hline Calothrix crustacea & Sargasso Sea & $\mathrm{R}$ & M & Ryland 1974 \\
\hline Calothrix parietina & Sargasso Sea & $\mathrm{R}$ & M & Conover \& Sieburth 1964 \\
\hline Calothrix sp. & Sargasso Sea & $\mathrm{R}$ & M & Hentschel 1922 \\
\hline Dichothrix bornetiana & Sargasso Sea & $\mathrm{R}$ & M & Conover \& Sieburth 1964 \\
\hline Dichothrix fucicola & $\begin{array}{l}\text { North Atlantic, Sargasso } \\
\text { Sea }\end{array}$ & $\mathrm{R}$ & M & $\begin{array}{l}\text { Carpenter 1972, Smith et al. } \\
\text { 1973, Carpenter \& Cox } 1974\end{array}$ \\
\hline Dichothrix sp. & Sargasso Sea & $\mathrm{R}$ & M & Hentschel 1922 \\
\hline Isactis sp. & Sargasso Sea & $\mathrm{R}$ & M & Hentschel 1922 \\
\hline Lyngbia majuscula & Sargasso Sea & $\mathrm{R}$ & M & $\begin{array}{l}\text { Phillips 1963, Conover \& } \\
\text { Sieburth } 1964\end{array}$ \\
\hline Oscillatoria subuliformis & Florida, Gulf of Mexico & $\mathrm{R}$ & $\mathrm{O}$ & Phillips 1963 \\
\hline Oscillatoria sp. & Belize & $\mathrm{R}$ & $\mathrm{O}$ & Faust \& Gulledge 1996 \\
\hline Spirulina subsalsa & Sargasso Sea & $\mathrm{R}$ & M & $\begin{array}{l}\text { Phillips 1963, Conover \& } \\
\text { Sieburth } 1964\end{array}$ \\
\hline \multicolumn{5}{|l|}{ Fungi } \\
\hline Aniptodera chesapeakensis & India & B & W & Prasannarai \& Sridhar 1997 \\
\hline Arenariomyces trifurcatus & $\begin{array}{l}\text { Malaysia/Singapore, } \\
\text { Hawaii \& Society Islands }\end{array}$ & $\mathrm{B}, \mathrm{D}$ & $\mathrm{W}$ & $\begin{array}{l}\text { Kohlmeyer 1984, Volkmann- } \\
\text { Kohlmeyer \& Kohlmeyer } \\
\text { 1993, Sundari et al. } 1996\end{array}$ \\
\hline Arenariomyces triseptatus & Hawaii \& Society Islands & $\mathrm{D}$ & $\mathrm{W}$ & $\begin{array}{l}\text { Volkmann-Kohlmeyer \& } \\
\text { Kohlmeyer } 1993\end{array}$ \\
\hline Caryosporella rhizophorae & India & $\mathrm{B}$ & W & Prasannarai \& Sridhar 1997 \\
\hline Corollospora cinnamomea & Hawaii \& Society Islands & $\mathrm{D}$ & $\mathrm{W}$ & $\begin{array}{l}\text { Volkmann-Kohlmeyer \& } \\
\text { Kohlmeyer } 1993\end{array}$ \\
\hline Corollospora colossa & Malaysia/Singapore, India & $\mathrm{B}$ & W & $\begin{array}{l}\text { Sundari et al. 1996, } \\
\text { Prasannarai \& Sridhar } 1997\end{array}$ \\
\hline Corollospora lacera & Hawaii \& Society Islands & $\mathrm{D}$ & $\mathrm{W}$ & $\begin{array}{l}\text { Volkmann-Kohlmeyer \& } \\
\text { Kohlmeyer } 1993\end{array}$ \\
\hline Corollospora maritima & Worldwide & $\mathrm{B}$ & $\mathrm{W}$ & $\begin{array}{l}\text { Kohlmeyer 1984, Sundari et al. } \\
1996\end{array}$ \\
\hline Corollospora pulchella & Worldwide & $\mathrm{B}$ & $\mathrm{W}$ & $\begin{array}{l}\text { Kohlmeyer 1984, Sundari et al. } \\
1996\end{array}$ \\
\hline Corollospora quinqueseptata & Hawaii \& Society Islands & $\mathrm{D}$ & $\mathrm{W}$ & $\begin{array}{l}\text { Volkmann-Kohlmeyer \& } \\
\text { Kohlmeyer } 1993\end{array}$ \\
\hline Crinigera maritima & India & $\mathrm{B}$ & W & Prasannarai \& Sridhar 1997 \\
\hline Dictyosporium pelagicum & India & $\mathrm{B}$ & $\mathrm{W}$ & Prasannarai \& Sridhar 1997 \\
\hline Didymosphaeria enalia & Worldwide & $\mathrm{B}$ & $\mathrm{W}$ & Kohlmeyer 1984 \\
\hline Halocyphina villosa & Brunei, South China Sea & $\mathrm{D}$ & $\mathrm{W}$ & Hyde 1989 \\
\hline Haloguignardia oceanica & North Carolina & $\mathrm{R}$ & M & Kohlmeyer 1972 \\
\hline Halosarpheia abonnis & Worldwide & B & W & Kohlmeyer 1984 \\
\hline Halosarpheia marina & Brunei, South China Sea & $\mathrm{D}$ & W & Hyde 1989 \\
\hline Halosarpheia salina & Brunei, South China Sea & $\mathrm{D}$ & $\mathrm{W}$ & Hyde 1989 \\
\hline Halosphaeria quadricornuta & Worldwide & B & $\mathrm{W}$ & Kohlmeyer 1984 \\
\hline Halosphaeria salina & Worldwide & B & W & Kohlmeyer 1984 \\
\hline Halosphaeriopsis mediosetigera & Worldwide & B & W & Kohlmeyer 1984 \\
\hline Humicola alopallonella & Worldwide & B & W & Kohlmeyer 1984 \\
\hline Lignincola laevis & Worldwide & B & W & Kohlmeyer 1984 \\
\hline
\end{tabular}




\section{MARTIN THIEL \& LARS GUTOW}

Table 2 (continued) Cyanobacteria, Fungi, Algae and Vascular plants reported or inferred as rafting

\begin{tabular}{|c|c|c|c|c|}
\hline Species & Region & $\begin{array}{l}\text { Rafting } \\
\text { inference }\end{array}$ & $\begin{array}{l}\text { Floating } \\
\text { substratum }\end{array}$ & Reference \\
\hline Lindra marinera & Worldwide & B & n.i. & Kohlmeyer 1984 \\
\hline Lindra thalassiae & Worldwide & $\mathrm{B}$ & $\mathrm{M}$ & Kohlmeyer 1984 \\
\hline Lulworthia grandispora & Worldwide & B & W & Kohlmeyer 1984 \\
\hline Periconia prolifica & Worldwide & $\mathrm{B}$ & W & Kohlmeyer 1984 \\
\hline Savoryella appendiculata & Malaysia/Singapore & $\mathrm{B}$ & W & Sundari et al. 1996 \\
\hline Sphaceloma cecidii & North Carolina & $\mathrm{R}$ & M & Kohlmeyer 1972 \\
\hline Varicosporina ramulosa & Worldwide & $\mathrm{B}$ & M & Kohlmeyer 1984 \\
\hline Verruculina enalia & India & $\mathrm{B}$ & W & Prasannarai \& Sridhar 1997 \\
\hline Zalerion varium & Worldwide & B & W & Kohlmeyer 1984 \\
\hline \multicolumn{5}{|l|}{ Dinoflagellata } \\
\hline Alexandrium taylori & Spain (Mediterranean) & B & $\mathrm{P}$ & Masó et al. 2003 \\
\hline Amphidinium klebsii & Florida Strait & $\mathrm{R}$ & M & Bomber et al. 1988 \\
\hline Amphidinium sp. & Belize & $\mathrm{R}$ & $\mathrm{O}$ & Faust \& Gulledge 1996 \\
\hline Coolia monotis & Florida Strait & $\mathrm{R}$ & M & Bomber et al. 1988 \\
\hline Coolia sp. & Spain (Mediterranean) & $\mathrm{B}$ & $\mathrm{P}$ & Masó et al. 2003 \\
\hline Gambierdiscus toxicus & $\begin{array}{l}\text { Florida Keys, Florida } \\
\text { Straight }\end{array}$ & $\mathrm{D}, \mathrm{R}$ & M & $\begin{array}{l}\text { Besada et al. 1982, Bomber } \\
\text { et al. } 1988\end{array}$ \\
\hline Ostreopsis heptagona & Florida Strait & $\mathrm{R}$ & M & Bomber et al. 1988 \\
\hline Ostreopsis siamensis & Florida Strait & $\mathrm{R}$ & M & Bomber et al. 1988 \\
\hline Ostreopsis sp. & Spain (Mediterranean) & $\mathrm{B}$ & $\mathrm{P}$ & Masó et al. 2003 \\
\hline Prorocentrum concavum & Florida Strait & $\mathrm{R}$ & M & Bomber et al. 1988 \\
\hline Prorocentrum emarginatum & Florida Strait & $\mathrm{R}$ & M & Bomber et al. 1988 \\
\hline Prorocentrum foraminosum & Belize & $\mathrm{R}$ & $\mathrm{O}$ & Faust \& Gulledge 1996 \\
\hline Prorocentrum hoffmannianum & Belize & $\mathrm{R}$ & $\mathrm{O}$ & Faust \& Gulledge 1996 \\
\hline Prorocentrum lima & Florida Strait & $\mathrm{R}$ & M & Bomber et al. 1988 \\
\hline Prorocentrum mexicanum & Florida Strait & $\mathrm{R}$ & M & Bomber et al. 1988 \\
\hline \multicolumn{5}{|l|}{ Chrysophyta } \\
\hline Cocconeis dirupta & Sargasso Sea & $\mathrm{R}$ & M & Carpenter 1970 \\
\hline Cyclotella meneghiniana & Sargasso Sea & $\mathrm{R}$ & $\mathrm{M}, \mathrm{P}$ & $\begin{array}{l}\text { Carpenter 1970, Carpenter \& } \\
\text { Smith } 1972\end{array}$ \\
\hline Fragilaria sp. & Falkland Current & $\mathrm{R}$ & M & Hentschel 1922 \\
\hline Licmophora sp. & Falkland Current & $\mathrm{R}$ & M & Hentschel 1922 \\
\hline Mastogloia angulata & Sargasso Sea & $\mathrm{R}$ & M, P & $\begin{array}{l}\text { Carpenter 1970, } \\
\text { Carpenter \& Smith } 1972\end{array}$ \\
\hline Mastogloia binotata & Sargasso Sea & $\mathrm{R}$ & M & Carpenter 1970 \\
\hline Mastogloia erythraea & Sargasso Sea & $\mathrm{R}$ & M & Carpenter 1970 \\
\hline Mastogloia gomphonemoides & Sargasso Sea & $\mathrm{R}$ & M & Carpenter 1970 \\
\hline Mastogloia guillardi & Sargasso Sea & $\mathrm{R}$ & M & Carpenter 1970 \\
\hline Mastogloia hulburti & Sargasso Sea & $\mathrm{R}$ & $\mathrm{M}, \mathrm{P}$ & $\begin{array}{l}\text { Carpenter 1970, Carpenter \& } \\
\text { Smith } 1972\end{array}$ \\
\hline Mastogloia ovulum & Sargasso Sea & $\mathrm{R}$ & M & Carpenter 1970 \\
\hline Mastogloia pusilla & Sargasso Sea & $\mathrm{R}$ & M & $\begin{array}{l}\text { Carpenter 1970, Carpenter \& } \\
\text { Smith } 1972\end{array}$ \\
\hline Pleurosigma sp. & Sargasso Sea & $\mathrm{R}$ & $\mathrm{M}, \mathrm{P}$ & $\begin{array}{l}\text { Carpenter 1970, Carpenter \& } \\
\text { Smith } 1972\end{array}$ \\
\hline Synedra sp. & Falkland Current & $\mathrm{R}$ & $\mathrm{M}$ & Hentschel 1922 \\
\hline
\end{tabular}




\section{RAFTING OF BENTHIC MARINE ORGANISMS}

Table 2 (continued) Cyanobacteria, Fungi, Algae and Vascular plants reported or inferred as rafting

\begin{tabular}{|c|c|c|c|c|}
\hline Species & Region & $\begin{array}{l}\text { Rafting } \\
\text { inference }\end{array}$ & $\begin{array}{l}\text { Floating } \\
\text { substratum }\end{array}$ & Reference \\
\hline \multicolumn{5}{|l|}{ Chlorophyta } \\
\hline Caulerpa flexilis & Tasmania & $\mathrm{R}$ & M & Edgar 1987 \\
\hline Caulerpa simpliciuscula & Tasmania & $\mathrm{R}$ & M & Edgar 1987 \\
\hline Caulerpa trifaria & Tasmania & $\mathrm{R}$ & M & Edgar 1987 \\
\hline Cladophora laetevirens & Azores & $\mathrm{C}$ & M & Morton \& Britton 2000 \\
\hline Colpomenia peregrina & California & $\mathrm{R}$ & $\mathrm{O}$ & Worcester 1994 \\
\hline Derbesia marina & Brazil Current & $\mathrm{R}$ & M & Oliveira et al. 1979 \\
\hline Enteromorpha chaetomorphoides & Brazil Current & $\mathrm{R}$ & M & Oliveira et al. 1979 \\
\hline Enteromorpha sp. & California & $\mathrm{R}$ & $\mathrm{O}$ & Worcester 1994 \\
\hline Enteromorpha sp. & Tasmania & $\mathrm{R}$ & M & Edgar 1987 \\
\hline Monostroma sp. & Falkland Current & $\mathrm{R}$ & M & Hentschel 1922 \\
\hline Ulva rigida & Azores & $\mathrm{C}$ & $\mathrm{P}, \mathrm{W}$ & Morton \& Britton 2000 \\
\hline Ulva sp. & California & $\mathrm{R}$ & $\mathrm{O}$ & Worcester 1994 \\
\hline Ulva sp. & Tasmania & $\mathrm{R}$ & M & Edgar 1987 \\
\hline \multicolumn{5}{|l|}{ Phaeophyta } \\
\hline Acrocarpia paniculata & Tasmania & $\mathrm{R}$ & M & Edgar 1987 \\
\hline Ascophyllum nodosum & Maine & $\mathrm{C}$ & n.i. & Dudgeon et al. 2001 \\
\hline Carpoglossum confluens & Tasmania & $\mathrm{R}$ & M & Edgar 1987 \\
\hline Colpomenia sinuosa & Brazil Current & $\mathrm{R}$ & M & Oliveira et al. 1979 \\
\hline Cystophora retroflexa & Tasmania & $\mathrm{R}$ & M & Edgar 1987 \\
\hline Cystophora sp. & Tasmania & $\mathrm{R}$ & M & Edgar 1987 \\
\hline Cystoseira sp. & Mediterranean & $\mathrm{R}$ & $\mathrm{P}$ & Aliani \& Molcard 2003 \\
\hline Dictyota dichotoma & Tasmania & $\mathrm{R}$ & M & Edgar 1987 \\
\hline Dictyota mertensii & Brazil Current & $\mathrm{R}$ & M & Oliveira et al. 1979 \\
\hline Ecklonia radiata & Tasmania & $\mathrm{R}$ & M & Edgar 1987 \\
\hline Ectocarpus rhodochortonoides & Brazil Current & $\mathrm{R}$ & M & Oliveira et al. 1979 \\
\hline Ectocarpus sp. & Falkland Current & $\mathrm{R}$ & M & Hentschel 1922 \\
\hline Elachistea minutissima & Brazil Current & $\mathrm{R}$ & M & Oliveira et al. 1979 \\
\hline Fucus vesiculosus & New England & $\mathrm{R}$ & $\mathrm{O}$ & Hardwick-Witman 1985 \\
\hline Giffordia rallsiae & Brazil Current & $\mathrm{R}$ & M & Oliveira et al. 1979 \\
\hline Halopteris sp. & Tasmania & $\mathrm{R}$ & M & Edgar 1987 \\
\hline Sargassum sp. & Tasmania & $\mathrm{R}$ & M & Edgar 1987 \\
\hline Sphacelaria furcigera & Brazil Current & $\mathrm{R}$ & M & Oliveira et al. 1979 \\
\hline Xiphophora gladiata & Tasmania & $\mathrm{R}$ & M & Edgar 1987 \\
\hline \multicolumn{5}{|l|}{ Rhodophyta } \\
\hline Amphiroa sp. & Bermuda & B & $\mathrm{P}$ & Winston et al. 1997 \\
\hline Archaetium sargassi & Brazil Current & $\mathrm{R}$ & M & Oliveira et al. $1979^{*}$ \\
\hline Ballia callitricha & Tasmania & $\mathrm{R}$ & M & Edgar 1987 \\
\hline Ballia scoparia & Tasmania & $\mathrm{R}$ & M & Edgar 1987 \\
\hline Callophyllis rangiferinus & Tasmania & $\mathrm{R}$ & M & Edgar 1987 \\
\hline Ceramium dawsoni & Brazil Current & $\mathrm{R}$ & M & Oliveira et al. 1979 \\
\hline Ceramium luetzelburgii & Brazil Current & $\mathrm{R}$ & M & Oliveira et al. 1979 \\
\hline Ceramium sp. & Tasmania & $\mathrm{R}$ & M & Edgar 1987 \\
\hline Ceramium sp. & Sargasso Sea & $\mathrm{R}$ & M & Hentschel 1922 \\
\hline Champia sp. & Tasmania & $\mathrm{R}$ & M & Edgar 1987 \\
\hline Chondria platyramea & Brazil Current & $\mathrm{R}$ & M & Oliveira et al. 1979 \\
\hline
\end{tabular}




\section{MARTIN THIEL \& LARS GUTOW}

Table 2 (continued) Cyanobacteria, Fungi, Algae and Vascular plants reported or inferred as rafting

\begin{tabular}{|c|c|c|c|c|}
\hline Species & Region & $\begin{array}{l}\text { Rafting } \\
\text { inference }\end{array}$ & $\begin{array}{l}\text { Floating } \\
\text { substratum }\end{array}$ & Reference \\
\hline Chondria polyrhiza & Brazil Current & $\mathrm{R}$ & M & Oliveira et al. 1979 \\
\hline Crouania attenuata & Brazil Current & $\mathrm{R}$ & M & Oliveira et al. 1979 \\
\hline Dasyclonium incisum & Tasmania & $\mathrm{R}$ & M & Edgar 1987 \\
\hline Delisea hypneoides & Tasmania & $\mathrm{R}$ & M & Edgar 1987 \\
\hline Delisea pulchra & Tasmania & $\mathrm{R}$ & M & Edgar 1987 \\
\hline Diplothamnion tetrastichum & Brazil Current & $\mathrm{R}$ & M & Oliveira et al. 1979 \\
\hline Dohrnellia antillarum & Brazil Current & $\mathrm{R}$ & M & Oliveira et al. 1979 \\
\hline Enteromorpha chaetomorphoides & Brazil Current & $\mathrm{R}$ & M & Oliveira et al. 1979 \\
\hline Erythrotrichia carnea & Brazil Current & $\mathrm{R}$ & M & Oliveira et al. 1979 \\
\hline Euptilota articulata & Tasmania & $\mathrm{R}$ & M & Edgar 1987 \\
\hline Falkenbergia hildenbrandii & Brazil Current & $\mathrm{R}$ & M & Oliveira et al. 1979 \\
\hline Fosliella atlantica & Brazil Current & $\mathrm{R}$ & M & Oliveira et al. 1979 \\
\hline Fosliella farinosa & Mediterranean & $\mathrm{R}$ & $\mathrm{P}$ & Aliani \& Molcard 2003 \\
\hline Fosliella sp. & Bermuda & B & $\mathrm{P}$ & Winston et al. 1997 \\
\hline Griffithsia schousboei & Brazil Current & $\mathrm{R}$ & M & Oliveira et al. 1979 \\
\hline Hemineura frondosa & Tasmania & $\mathrm{R}$ & M & Edgar 1987 \\
\hline Herposiphonia secunda & Brazil Current & $\mathrm{R}$ & M & Oliveira et al. 1979 \\
\hline Herposiphonia sp. & Azores & $\mathrm{C}$ & M & Morton \& Britton 2000 \\
\hline Heterosiphonia wurdemannii & Brazil Current & $\mathrm{R}$ & M & Oliveira et al. 1979 \\
\hline Hypnea episcopalis & Tasmania & $\mathrm{R}$ & M & Edgar 1987 \\
\hline Hypoglossum tenuifolium & Brazil Current & $\mathrm{R}$ & M & Oliveira et al. 1979 \\
\hline Jania cappilacea & Brazil Current & $\mathrm{R}$ & M & Oliveira et al. 1979 \\
\hline Jania sp. & Bermuda & B & $\mathrm{P}$ & Winston et al. 1997 \\
\hline Jeanerretia lobata & Tasmania & $\mathrm{R}$ & M & Edgar 1987 \\
\hline Laurencia nana & Brazil Current & $\mathrm{R}$ & M & Oliveira et al. 1979 \\
\hline Lenormandia marginata & Tasmania & $\mathrm{R}$ & M & Edgar 1987 \\
\hline Lithophyllum sp. & Bermuda & B & $\mathrm{P}$ & Winston et al. 1997 \\
\hline Melobesia sp. & Sargasso Sea & $\mathrm{R}$ & M & $\begin{array}{l}\text { Hentschel 1922, Niermann } \\
1986\end{array}$ \\
\hline Mesophyllum sp. & Bermuda & B & $\mathrm{P}$ & Winston et al. 1997 \\
\hline Phacelocarpus labillardieri & Tasmania & $\mathrm{R}$ & M & Edgar 1987 \\
\hline Plocamium angustum & Tasmania & $\mathrm{R}$ & M & Edgar 1987 \\
\hline Plocamium dilatatum & Tasmania & $\mathrm{R}$ & M & Edgar 1987 \\
\hline Polysiphonia lanosa & Azores, Bermuda & $\mathrm{R}$ & M & John 1974, Ingólfsson 1998 \\
\hline Polysiphonia sp. & Tasmania & $\mathrm{R}$ & M & Edgar 1987 \\
\hline Polysiphonia subtilissima & Brazil Current & $\mathrm{R}$ & M & Oliveira et al. 1979 \\
\hline Sonderopelta coriacea & Tasmania & $\mathrm{R}$ & M & Edgar 1987 \\
\hline \multicolumn{5}{|l|}{ Bryophyta } \\
\hline Hypnum polare & Off Northeast Canada & $\mathrm{R}$ & $\mathrm{O}$ & $\begin{array}{l}\text { Polunin 1955, cited in } \\
\text { Johansen \& Hytteborn } 2001\end{array}$ \\
\hline \multicolumn{5}{|l|}{ Magnoliopsida } \\
\hline Acacia sp. & Hawaii & $\mathrm{D}$ & n.i. & Carlquist 1967 \\
\hline Canavalia sp. & Hawaii & $\mathrm{D}$ & n.i. & Carlquist 1967 \\
\hline Capparis sp. & Hawaii & $\mathrm{D}$ & n.i. & Carlquist 1967 \\
\hline Carex sp. & East Greenland & $\mathrm{B}$ & $\mathrm{W}$ & $\begin{array}{l}\text { Ingvarson 1903, cited in } \\
\text { Johansen \& Hytteborn } 2001\end{array}$ \\
\hline
\end{tabular}




\section{RAFTING OF BENTHIC MARINE ORGANISMS}

Table 2 (continued) Cyanobacteria, Fungi, Algae and Vascular plants reported or inferred as rafting

\begin{tabular}{|c|c|c|c|c|}
\hline Species & Region & $\begin{array}{l}\text { Rafting } \\
\text { inference }\end{array}$ & $\begin{array}{l}\text { Floating } \\
\text { substratum }\end{array}$ & Reference \\
\hline Cassia sp. & Hawaii & $\mathrm{D}$ & n.i. & Carlquist 1967 \\
\hline Cymodocea nodosa & Mediterranean & $\mathrm{R}$ & $\mathrm{P}$ & Aliani \& Molcard 2003 \\
\hline Dodonaea sp. & Hawaii & $\mathrm{D}$ & n.i. & Carlquist 1967 \\
\hline Draba siberica & Siberia & $\mathrm{D}$ & $\mathrm{O}$ & Johansen \& Hytteborn 2001 \\
\hline Erythrina sp. & Hawaii & $\mathrm{D}$ & n.i. & Carlquist 1967 \\
\hline Gossypium sp. & Hawaii & $\mathrm{D}$ & n.i. & Carlquist 1967 \\
\hline Gouania sp. & Hawaii & $\mathrm{D}$ & n.i. & Carlquist 1967 \\
\hline Haplostachys sp. & Hawaii & $\mathrm{D}$ & n.i. & Carlquist 1967 \\
\hline Hibiscadelphus sp. & Hawaii & $\mathrm{D}$ & n.i. & Carlquist 1967 \\
\hline Hibiscus sp. & Hawaii & $\mathrm{D}$ & n.i. & Carlquist 1967 \\
\hline Jacquemontia sp. & Hawaii & $\mathrm{D}$ & n.i. & Carlquist 1967 \\
\hline Kokia sp. & Hawaii & $\mathrm{D}$ & n.i. & Carlquist 1967 \\
\hline Luzula sp & East Greenland & B & $\mathrm{W}$ & $\begin{array}{l}\text { Ingvarson 1903, cited in } \\
\text { Johansen \& Hytteborn } 2001\end{array}$ \\
\hline Morinda sp. & Hawaii & $\mathrm{D}$ & n.i. & Carlquist 1967 \\
\hline Ochrosia sp. & Hawaii & $\mathrm{D}$ & n.i. & Carlquist 1967 \\
\hline Oxytropis deflexa & Siberia & $\mathrm{D}$ & $\mathrm{O}$ & Johansen \& Hytteborn 2001 \\
\hline Peиcedanum sp. & Hawaii & $\mathrm{D}$ & n.i. & Carlquist 1967 \\
\hline Phippsia algida & Off Northeast Canada & $\mathrm{R}$ & $\mathrm{O}$ & $\begin{array}{l}\text { Hultén 1962, cited in Johansen } \\
\text { \& Hytteborn } 2001\end{array}$ \\
\hline Posidonia oceanica & Mediterranean & $\mathrm{R}$ & $\mathrm{P}$ & Aliani \& Molcard 2003 \\
\hline Potentilla stipularis & Siberia & $\mathrm{D}$ & $\mathrm{O}$ & Johansen \& Hytteborn 2001 \\
\hline Potentilla sp. & East Greenland & B & $\mathrm{W}$ & $\begin{array}{l}\text { Ingvarson 1903, cited in } \\
\text { Johansen \& Hytteborn } 2001\end{array}$ \\
\hline Pteralyxia sp. & Hawaii & $\mathrm{D}$ & n.i. & Carlquist 1967 \\
\hline Rumex sp. & Hawaii & $\mathrm{D}$ & n.i. & Carlquist 1967 \\
\hline Salicornia virginica & California & $\mathrm{R}$ & $\mathrm{O}$ & Worcester 1994 \\
\hline Saxifraga oppositifolia & Off Northeast Canada & $\mathrm{R}$ & $\mathrm{O}$ & $\begin{array}{l}\text { Hultén 1962, cited in Johansen } \\
\text { \& Hytteborn } 2001\end{array}$ \\
\hline Sesbania sp. & Hawaii & $\mathrm{D}$ & n.i. & Carlquist 1967 \\
\hline Sida sp. & Hawaii & $\mathrm{D}$ & n.i. & Carlquist 1967 \\
\hline Sophora sp. & Hawaii & D & n.i. & Carlquist 1967 \\
\hline Spartina alterniflora & New England & $\mathrm{R}$ & $\mathrm{O}$ & Hardwick-Witman 1985 \\
\hline Spartina foliosa & California & $\mathrm{R}$ & $\mathrm{O}$ & Worcester 1994 \\
\hline Stellaria laeta & Off Northeast Canada & $\mathrm{R}$ & $\mathrm{O}$ & $\begin{array}{l}\text { Hultén 1962, cited in Johansen } \\
\text { \& Hytteborn } 2001\end{array}$ \\
\hline Trisetum subalpestre & Siberia & $\mathrm{D}$ & $\mathrm{O}$ & Johansen \& Hytteborn 2001 \\
\hline Waltheria sp. & Hawaii & $\mathrm{D}$ & n.i. & Carlquist 1967 \\
\hline Zostera marina & Nova Scotia & $\mathrm{R}$ & $\mathrm{O}$ & $\begin{array}{r}\text { Schneider \& Mann 1991, cited } \\
\text { in Johansen \& Hytteborn } 2001\end{array}$ \\
\hline
\end{tabular}

Notes: $\mathrm{B}=$ stranded floating item on beach; $\mathrm{C}=$ circumstantial; $\mathrm{D}=$ distributional inference; $\mathrm{M}=$ macroalgae; n.i. $=$ no information; $\mathrm{O}=$ other; $\mathrm{P}=$ plastics; $\mathrm{R}=$ rafting; $\mathrm{W}=$ wood.

* References refer to this species under a different name. 


\section{MARTIN THIEL \& LARS GUTOW}

members of the biofilm. Cundell et al. (1978) analysed microbial populations associated with the surface of the brown alga Ascophyllum nodosum and described the antibacterial activity of floating plants. Cyanobacteria also represent an important component of floating detritus matrices (Phillips 1963, Faust \& Gulledge 1996).

\section{Fungi}

Various marine fungi growing on wood and algae have been suggested to be transported via rafting (Kohlmeyer 1984, Hyde 1989, Sundari et al. 1995, 1996, Prasannarai \& Sridhar 1997) (Table 2). Reports of rafting fungi are not surprising since one third of all described marine fungi occur on algae (Kohlmeyer 1972). Accidental rafting of fungal organisms can also be the result of the intricate symbiotic relationships found in this group. Parasitic and hyperparasitic fungi, which may be dispersed simultaneously, have been described from Sargassum natans by Kohlmeyer (1972). In the tropics many fungal species were collected from stranded Sargassum spp. on which they are often found in empty bryozoan skeletons (Kohlmeyer 1984).

Many lignicolous and arenicolous fungi isolated from wooden substrata have a cosmopolitan distribution (Kohlmeyer 1984, Sundari et al. 1996). The distribution pattern of these fungi might be a result of the high buoyancy of their primary substrata. Driftwood might become inoculated with fungi after having been cast ashore on sandy beaches. Spores of various arenicolous fungal species found in marine foam in the tropics (Kohlmeyer 1984) are likely to colonise stranded wood. During storm events with high wave surge, this wood may become waterborne again and be carried with currents to new areas, as has been suggested by Dyke et al. (1997). This process may lead to efficient dispersal of wood-dwelling fungi resulting in their cosmopolitan distribution (e.g., Sundari et al. 1996).

Wood-dwelling fungi are often found in empty tubes of teredinid bivalves or limnorid isopods (Kohlmeyer 1984). Marine fungi (and bacteria) have been shown to essentially precondition wood, allowing access of other wood-boring organisms (Kampf et al. 1959 cited in Kohlmeyer et al. 1995). Larvae of teredinids, for example, are incapable of penetrating submerged wood as long as the bark is intact. Lignicolous fungi often require incubation times of several months before they fruit on driftwood making them identifiable (Kohlmeyer et al. 1995, Prasannarai \& Sridhar 1997).

A surprisingly low number of mangroves have been found to support marine fungi. Even though the plants are in permanent contact with sea water, apparently only a low proportion $(<10 \%)$ of the species serve as host for marine fungi (Kohlmeyer \& Kohlmeyer 1979 cited in Kohlmeyer 1984). Uprooted mangroves or parts thereof, which are commonly found as driftwood (e.g., Hyde 1989, Si et al. 2000), may serve as dispersal vectors for associated fungi.

Fungi themselves are indicators of decomposition processes. At present, little is known of the effects that fungi have on the survival of their substrata at the sea surface. Buoyancy of wood or macroalgae infested by fungi might be relatively limited.

\section{Microalgae}

Diatoms have been found attached to floating Sargassum (Hentschel 1922, Carpenter 1970), to plastic spherules (Carpenter \& Smith 1972, Gregory 1978) and to tar balls (Wong et al. 1974) (Table 2). Gregory (1978) reported diatoms and coralline algae on plastic pellets from New Zealand beaches. Due to their small biomass compared with other epiphytic algae, diatoms were expected by Smith et al. (1973) to contribute only a small amount to the metabolism of the community on

floating Sargassum. Consequently, the authors considered diatoms on Sargassum as functionally unimportant. Moore et al. (2001) found diatoms and other microalgae on a monofilament plastic 


\section{RAFTING OF BENTHIC MARINE ORGANISMS}

line about $10 \mathrm{~m}$ below the surface of the central North Pacific. They assumed that overgrowth with micro-organisms affected the buoyancy of the plastic line and caused it to sink. Benthic microalgae (including diatoms and dinoflagellates) may also form a dense matrix, which entraps gas bubbles during the day and then floats to the surface, carrying with it a wide diversity of microalgae (Phillips 1963, Faust \& Gulledge 1996).

Rafting dinoflagellates have been primarily reported from floating macroalgae and plastics (Table 2). Non-buoyant stages in the life cycle of marine microalgae may attach to floating substrata (Masó et al. 2003). For example, temporary cysts of dinoflagellates (Alexandrium taylori) have been found from a variety of floating plastic items (Masó et al. 2003). Bomber et al. (1988) reported nine dinoflagellate species as epiphytes of floating macroalgae. Dinoflagellates were collected from about $65 \%$ of all floating macroalgae (mainly Sargassum natans and S. fluitans) collected in the Florida Keys region. One of the species found was the benthic dinoflagellate Gambierdiscus toxicus, a ciguatoxin-producing species, for which Besada et al. (1982), based on its poor swimming abilities and non-planktonic habit, concluded that rafting may be its major dispersal mechanism and help explain some of its patchy distributions. Thus, as suggested by Hallegraeff (1992) and Masó et al. (2003), it appears possible that toxic microalgae could also become dispersed via rafting on natural and anthropogenic floating substrata.

\section{Macroalgae}

Some macroalgae are positively buoyant and may float over variable distances (Thiel \& Gutow 2004). Most algae, though, are negatively buoyant and may raft as epiphytes on floating macroalgal species (Woelkerling 1975, Hoek 1987) or on other floating items (Winston et al. 1997, Aliani \& Molcard 2003) (Table 2). Besides several self-buoyant macroalgae, Stegenga \& Mol (1983) mentioned large numbers of allochthonous, non-buoyant algae with southerly origin (Normandy, Brittany, southern England) that were found on beaches of the Netherlands. These algae grew attached to other floating substrata such as wood, cork and plastic. Edgar (1987) listed a large number of macroalgal species associated with detached holdfasts of Macrocystis pyrifera several months after detachment of the latter. Even large species that are also buoyant such as Ecklonia radiata were found growing on algal substrata. Mitchell \& Hunter (1970) reported some non-buoyant brown algae entangled among rafts of Macrocystis pyrifera. Large numbers of epiphytic algae had also been found on floating Sargassum off the coast of Brazil (Oliveira et al. 1979), and Ingólfsson (1998) mentioned that the red alga Polysiphonia lanosa is a common epiphyte on Ascophyllum nodosum found floating in Icelandic waters. Kornicker \& Squires (1962) observed algae growing on floating corals that had recently been cast ashore but they did not specify the algae. At the Great Barrier Reef mainly crustose coralline and filamentous blue-green algae rafted on floating corals (DeVantier 1992). Very similar observations have been made for macroalgae on floating volcanic pumice (Jokiel 1989).

The spores of many macroalgae have a very limited dispersal potential and may be dispersed over only a few metres, while adult plants or parts thereof may float over large distances and either reattach or release spores near new habitats (e.g., Hoffmann 1987, but see also Reed et al. 1988, 1992). Spores of macroalgae may thus raft on the parent plant. Distributional evidence was used by Hoek (1987) to infer that the algal flora of some oceanic islands has arrived via rafting. He demonstrated that long-range dispersal of seaweeds by planktonic propagules such as spores could be neglected since the lifetime of these developmental stages is too short for them to reach distant shores. The importance of floating and rafting for long-distance dispersal of macroalgae is underscored by two facts, namely that (1) island flora in many cases is dominated by algal species that are positively buoyant and that (2) distances from potential sources are far beyond the dispersal range of spores (Hoek 1987). 


\section{MARTIN THIEL \& LARS GUTOW}

\section{Vascular plants}

Many higher plants or their seeds possess positive buoyancy and may float autonomously over long time periods and large distances (Thiel \& Gutow 2004). Fragments of aquatic vascular plants have often been found interspersed with floating Sargassum in the North Atlantic (Dooley 1972). Most higher plants, though, are not buoyant and consequently depend on floating substrata to be transported via the sea surface (Table 2). There exist anecdotal reports that uprooted trees floating at the sea surface carried in their root system terrestrial soil that may have contained seeds of other plants (Hemsley 1885). Entire floating islands have been observed with large nipa palms entangled in them being mistaken for a three-masted vessel (Carlquist 1965). These islands are created by floods undermining the matted roots of riverside nipas (St. John 1862 cited in Brandon-Jones 1998). Several authors suggested that members of the terrestrial flora might have become dispersed via rafting (Guppy 1906, Carlquist 1967, Johansen \& Hytteborn 2001). In many cases, the exact mechanisms are not known, though. It appears justified to assume that beach and estuarine flora are most likely to appear on a raft. To our knowledge, no published reports of non-buoyant flora on rafts are available. Muir (1934) suggested that the occurrence of terrestrial plant seeds, which themselves are non-buoyant, on South African beaches might be the result of rafting on pieces of pumice or floating corals. Plants or seeds of marine plants can also become dispersed via currents (Koutstaal et al. 1987). The seeds of most mangroves have only limited buoyancy (e.g., Steinke 1986), but Rhizophora (Duke 1995) as well as Avicennia nitida (Guppy 1917) are considered as dispersal specialists because their seeds can float for long time periods (see also Steinke \& Ward 2003). Several authors have inferred that seagrasses may have colonised distant shores via rafting of seeds in floating shoots (González \& Edding 1990a, Harwell \& Orth 2002). The dispersal distances of seeds of the seagrass Zostera marina appear to be limited (Orth et al. 1994, Ruckelshaus 1996, Kaldy \& Dunton 1999). In Chesapeake Bay, Orth et al. (1994) frequently observed reproductive, seed-carrying shoots of $Z$. marina that were floating at the sea surface, and they suggested that long-distance dispersal and colonisation of distant habitats may be achieved via these floating plants. This suggestion was supported by subsequent experiments showing that seed-bearing shoots remained afloat for 2-3 wk (Harwell \& Orth 2002). While this is not rafting sensu strictu, dispersal potential of seeds is increased via transport on adult shoots, similar to the mechanism suggested for macroalgae spores (see above). In New England salt marshes short-distance ice rafting has been observed for Spartina alterniflora together with intertidal peat patches frozen to ice blocks (HardwickWitman 1985).

For one species of moss, rafting has also been suggested as a potential dispersal mechanism (Polunin 1955 cited in Johansen \& Hytteborn 2001). Delgadillo (2000) suggested that migration may occur between continental America and the Caribbean Islands, but he did not elaborate on a potential dispersal mechanism. Since mosses frequently grow on the trunk of old trees, it is not considered unlikely that mosses can be dispersed via rafting.

\section{Protists}

Foraminiferans and other protists are often found to inhabit abiotic substrata (Table 3). Chirat (2000) reported foraminiferans growing on floating shells of Nautilus while DeVantier (1992) found foraminiferans on buoyant skeletons of tropical reef corals. Spindler (1980) mentioned the foraminiferans Planorbulina acervalis and Rosalina globularis on floating Sargassum. Dow \& Menzies (1958) and Herring (1969) collected radiolarians in the gut of Idotea metallica, but provided no information whether this obligate rafter obtained these protists from the surrounding water or from its floating substratum. Faust \& Gulledge (1996) observed large numbers of ciliates in a matrix of floating detritus. 


\section{RAFTING OF BENTHIC MARINE ORGANISMS}

Table 3 Protists and porifera reported or inferred as rafting

\begin{tabular}{|c|c|c|c|c|}
\hline Species & Region & $\begin{array}{l}\text { Rafting } \\
\text { inference }\end{array}$ & $\begin{array}{l}\text { Floating } \\
\text { substratum }\end{array}$ & Reference \\
\hline \multicolumn{5}{|l|}{ Foraminifera } \\
\hline Acervulina sp. & Florida & $\mathrm{B}$ & $P$ & Winston et al. 1997 \\
\hline Homotrema rubra & New Zealand & B & $\mathrm{P}$ & Winston et al. 1997 \\
\hline $\begin{array}{l}\text { Planorbulina } \\
\text { acervalis }\end{array}$ & Sargasso Sea & $\mathrm{R}$ & M & $\begin{array}{l}\text { Butler et al. 1983, } \\
\text { Coston-Clements et al. } 1991\end{array}$ \\
\hline Rosalina globularis & Sargasso Sea & $\mathrm{R}$ & M & Spindler 1980 \\
\hline Rosalina sp. & Sargasso Sea & $\mathrm{R}$ & M & $\begin{array}{l}\text { Butler et al. 1983, } \\
\text { Coston-Clements et al. } 1991\end{array}$ \\
\hline \multicolumn{5}{|l|}{ Ciliophora } \\
\hline Coleps sp. & Belize & $\mathrm{R}$ & $\mathrm{O}$ & Faust \& Gulledge 1996 \\
\hline Euplotes sp. & Belize & $\mathrm{R}$ & $\mathrm{O}$ & Faust \& Gulledge 1996 \\
\hline \multicolumn{5}{|l|}{ Porifera } \\
\hline Aplysina fistularis & California & $\mathrm{R}$ & M & Bushing 1994 \\
\hline Callyspongia sp. & Sagami Bay, Japan & $\mathrm{R}$ & M & Hirosaki 1964 \\
\hline Mycale plumosa & Sagami Bay, Japan & $\mathrm{R}$ & M & Hirosaki 1964 \\
\hline
\end{tabular}

Notes: $\mathrm{B}=$ stranded floating item on beach; $\mathrm{M}=$ macroalgae; $\mathrm{O}=$ other; $\mathrm{P}=$ plastics; $\mathrm{R}=$ rafting.

\section{Porifera}

Sponges have been recorded from floating macroalgae (Table 3). Among a diverse rafting fauna, Hirosaki (1964) also reported three different sponge species from floating Sargassum. Similarly, Bushing (1994) mentioned sponges from several other floating macroalgae collected in waters off southern California. Sponges may also grow abundantly on plastics anchored in coastal areas (e.g., buoys) and be carried with these after detachment (e.g., after storms). The same applies to natural substrata growing in coastal habitats such as macroalgae or mangrove roots. Aerial roots of the mangrove Rhizophora mangle host a rich fauna including diverse sponge species (Bingham \& Young 1995), which after breakage or uprooting of a mangrove tree may be transported via rafting. Winston et al. (1997) also reported unidentified sponges from floating plastics.

\section{Cnidaria (Hydrozoa)}

The most commonly reported rafters belong to the Hydrozoa (Table 4). While many Hydrozoa possess pelagic dispersal stages (e.g., Gravier-Bonnet 1992), other species lack medusae and therefore are thought to be less efficient dispersers (Cornelius 1992a,b). Some of these latter species, nevertheless, have a wide geographic distribution, suggesting efficient dispersal via rafting (Cornelius 1992a). Hydrozoans have indeed been reported from various floating substrata including macroalgae and plastic items (Carpenter \& Smith 1972, Butler et al. 1983, Calder 1995, Hobday 2000a, Barnes \& Sanderson 2000, Aliani \& Molcard 2003, Masó et al. 2003). Hydrozoans, which completely had overgrown potentially floating shells of dead nautiloids, have been reported as fossils (Chirat 2000).

Morris \& Mogelberg (1973) considered hydroids as the most diverse (43 species) and among the most common of all taxa associated with floating Sargassum. Hentschel (1922) reported several 


\section{MARTIN THIEL \& LARS GUTOW}

Table 4 Cnidaria reported or inferred as rafting

\begin{tabular}{|c|c|c|c|c|}
\hline Species & Region & $\begin{array}{l}\text { Rafting } \\
\text { inference }\end{array}$ & $\begin{array}{l}\text { Floating } \\
\text { substratum }\end{array}$ & Reference \\
\hline \multicolumn{5}{|l|}{ Hydrozoa } \\
\hline $\begin{array}{l}\text { Aglaophenia } \\
\text { latecarinata }\end{array}$ & $\begin{array}{l}\text { Sargasso Sea, } \\
\text { Northwest } \\
\text { Atlantic, Bermuda }\end{array}$ & $\mathrm{R}$ & M & $\begin{array}{l}\text { Hentschel 1922, Timmermann 1932, } \\
\text { Adams 1960, Butler et al. 1983, } \\
\text { Coston-Clements et al. 1991, } \\
\text { Calder } 1995\end{array}$ \\
\hline Aglaophenia perpusilla & $\begin{array}{l}\text { Sargasso Sea, North } \\
\text { Atlantic }\end{array}$ & $\mathrm{R}$ & M & $\begin{array}{l}\text { Smith et al. 1973, Butler et al. 1983, } \\
\text { Coston-Clements et al. } 1991\end{array}$ \\
\hline Aglaophenia pluma & Northwest Europe & $\mathrm{C}$ & M & Vallentin 1895, Cornelius 1992a \\
\hline Aglaophenia rigida & Sargasso Sea & $\mathrm{R}$ & M & $\begin{array}{l}\text { Butler et al. 1983, Coston-Clements } \\
\text { et al. } 1991\end{array}$ \\
\hline $\begin{array}{l}\text { Aglaophenoides } \\
\text { mammillatus }\end{array}$ & Sargasso Sea & $\mathrm{R}$ & M & $\begin{array}{l}\text { Butler et al. 1983, Coston-Clements } \\
\text { et al. } 1991\end{array}$ \\
\hline Amphisbetia pacifica & Japan & $\mathrm{R}$ & M & Sano et al. 2003 \\
\hline Antenella secundaria & $\begin{array}{l}\text { Sargasso Sea, } \\
\text { Northwest Europe }\end{array}$ & $\mathrm{D}, \mathrm{R}$ & M & $\begin{array}{l}\text { Butler et al. 1983, Coston-Clements } \\
\text { et al. 1991, Cornelius 1992a }\end{array}$ \\
\hline Campanularia gracilis & North Pacific & $\mathrm{R}$ & $\mathrm{O}$ & Sinitsyn \& Reznichenko 1981 \\
\hline Campanularia hincksii & Northwest Europe & $\mathrm{C}$ & n.i. & Cornelius 1992a \\
\hline Campanularia volubilis & Sargasso Sea & $\mathrm{R}$ & M & $\begin{array}{l}\text { Butler et al. 1983, Coston-Clements } \\
\text { et al. } 1991\end{array}$ \\
\hline Cladocoryne pelagica & Sargasso Sea & $\mathrm{R}$ & M & $\begin{array}{l}\text { Butler et al. 1983, Coston-Clements } \\
\text { et al. } 1991\end{array}$ \\
\hline Clava multicornis & n.i. & $\mathrm{R}$ & M & Vallentin 1895 \\
\hline Clytia bicophora & Sargasso Sea & $\mathrm{R}$ & M & Coston-Clements et al. 1991 \\
\hline Clytia cylindrica & Sargasso Sea & $\mathrm{R}$ & $\mathrm{M}, \mathrm{P}$ & $\begin{array}{l}\text { Timmermann 1932, Carpenter \& } \\
\text { Smith 1972, Butler et al. 1983, } \\
\text { Coston-Clements et al. } 1991\end{array}$ \\
\hline Clytia gracilis & Northwest Europe & $\mathrm{C}$ & n.i. & Cornelius 1992a \\
\hline Clytia hemisphaerica & $\begin{array}{l}\text { Northwest Europe, } \\
\text { Mediterranean, } \\
\text { Bermuda }\end{array}$ & $\mathrm{C}, \mathrm{R}$ & $\mathrm{M}, \mathrm{P}$ & $\begin{array}{l}\text { Cornelius 1992a, Calder 1995, } \\
\text { Aliani \& Molcard } 2003\end{array}$ \\
\hline Clytia johnstoni & $\begin{array}{l}\text { Sargasso Sea, Brazil } \\
\text { Current }\end{array}$ & $\mathrm{R}$ & M & $\begin{array}{l}\text { Hentschel 1922, Butler et al. 1983, } \\
\text { Coston-Clements et al. } 1991\end{array}$ \\
\hline Clytia linearis & Northwest Europe & $\mathrm{D}$ & n.i. & Cornelius 1992a \\
\hline Clytia longicyatha & Sargasso Sea & $\mathrm{R}$ & M & $\begin{array}{l}\text { Butler et al. 1983, Coston-Clements } \\
\text { et al. } 1991\end{array}$ \\
\hline Clytia noliformis & $\begin{array}{l}\text { Sargasso Sea, } \\
\text { Bermuda }\end{array}$ & $\mathrm{R}$ & M & $\begin{array}{l}\text { Hentschel 1922*, Ryland 1974, } \\
\text { Butler et al. 1983, Niermann 1986, } \\
\text { Coston-Clements et al. 1991, } \\
\text { Calder } 1995\end{array}$ \\
\hline Clytia raridentata & Sargasso Sea & $\mathrm{R}$ & M & $\begin{array}{l}\text { Butler et al. 1983, Coston-Clements } \\
\text { et al. } 1991\end{array}$ \\
\hline Clytia sp. & Northwest Atlantic & $\mathrm{R}$ & M & Adams 1960, Smith et al. 1973 \\
\hline Coryne pusilla & Japan & $\mathrm{R}$ & M & Sano et al. 2003 \\
\hline Dactylometra pacifica & Sagami Bay, Japan & $\mathrm{R}$ & M & Hirosaki 1964 \\
\hline Dynamena cornicina & Northwest Europe & $\mathrm{D}$ & n.i. & Cornelius 1992a \\
\hline Dynamena disticha & $\begin{array}{l}\text { Bermuda, } \\
\text { Northwest Europe }\end{array}$ & $\mathrm{R}$ & M & Cornelius 1992a, Calder 1995 \\
\hline Dynamena pumila & $\begin{array}{l}\text { Northwest Europe, } \\
\text { Iceland }\end{array}$ & $\mathrm{C}, \mathrm{R}$ & M & Cornelius 1992a, Ingólfsson 1995 \\
\hline
\end{tabular}




\section{RAFTING OF BENTHIC MARINE ORGANISMS}

Table 4 (continued) Cnidaria reported or inferred as rafting

\begin{tabular}{|c|c|c|c|c|}
\hline Species & Region & $\begin{array}{l}\text { Rafting } \\
\text { inference }\end{array}$ & $\begin{array}{l}\text { Floating } \\
\text { substratum }\end{array}$ & Reference \\
\hline $\begin{array}{l}\text { Dynamena } \\
\text { quadridentata }\end{array}$ & Sargasso Sea & $\mathrm{R}$ & M & $\begin{array}{l}\text { Butler et al. 1983, Coston-Clements } \\
\text { et al. } 1991\end{array}$ \\
\hline Eucopella sargassicola & Sargasso Sea & $\mathrm{R}$ & M & $\begin{array}{l}\text { Butler et al. 1983, Coston-Clements } \\
\text { et al. } 1991\end{array}$ \\
\hline Eudendrium sp. & Mediterranean & $\mathrm{R}$ & $\mathrm{P}$ & Aliani \& Molcard 2003 \\
\hline Filellum serpens & Northwest Europe & $\mathrm{C}$ & n.i. & Cornelius 1992a \\
\hline Filellum serratum & Northwest Europe & $\mathrm{D}$ & n.i. & Cornelius 1992a \\
\hline Gemmaria sp. & Sargasso Sea & $\mathrm{R}$ & M & Coston-Clements et al. 1991 \\
\hline Gonothyraea gracilis & Sargasso Sea & $\mathrm{R}$ & M & $\begin{array}{l}\text { Butler et al. 1983, Coston-Clements } \\
\text { et al. } 1991\end{array}$ \\
\hline Gonothyraea integra & Sargasso Sea & $\mathrm{R}$ & M & $\begin{array}{l}\text { Butler et al. 1983, Coston-Clements } \\
\text { et al. } 1991\end{array}$ \\
\hline Gonothyrea hyalina & Sargasso Sea & $\mathrm{R}$ & $\mathrm{P}$ & Carpenter \& Smith 1972 \\
\hline Gonothyrea loveni & Mediterranean & $\mathrm{R}$ & $\mathrm{P}$ & Aliani \& Molcard 2003 \\
\hline Halecium lankesteri & Northwest Europe & $\mathrm{C}$ & n.i. & Cornelius 1992a \\
\hline Halecium nanum & $\begin{array}{l}\text { Northwest Atlantic, } \\
\text { Sargasso Sea, } \\
\text { Bermuda }\end{array}$ & $\mathrm{R}$ & M & $\begin{array}{l}\text { Hentschel 1922, Timmermann 1932, } \\
\text { Adams 1960, Butler et al. 1983, } \\
\text { Coston-Clements et al. 1991, } \\
\text { Calder } 1995\end{array}$ \\
\hline Halecium tenellum & Northwest Europe & $\mathrm{C}$ & n.i. & Cornelius 1992a \\
\hline Halopteris catharina & Sargasso Sea & $\mathrm{D}, \mathrm{R}$ & M & $\begin{array}{l}\text { Timmermann } 1932 * \text {, Butler et al. } \\
1983^{*} \text {, Coston-Clements et al. } \\
1991 * \text {, Cornelius 1992a }\end{array}$ \\
\hline Halopteris diaphana & Bermuda & $\mathrm{R}$ & M & Calder 1995 \\
\hline Hebella calcarata & $\begin{array}{l}\text { Sargasso Sea, Brazil } \\
\text { Current }\end{array}$ & $\mathrm{R}$ & M & $\begin{array}{l}\text { Hentschel 1922, Butler et al. 1983, } \\
\text { Coston-Clements et al. } 1991\end{array}$ \\
\hline Kirchenpaueria pinnata & Northwest Europe & $\mathrm{C}$ & n.i. & Cornelius 1992a \\
\hline Kirchenраиеria similis & Northwest Europe & $\mathrm{D}$ & n.i. & Cornelius 1992a \\
\hline Lafoea dumosa & Northwest Europe & $\mathrm{C}$ & n.i. & Cornelius 1992a \\
\hline Lafoeina tenuis & Northwest Europe & $\mathrm{D}$ & n.i. & Cornelius 1992a \\
\hline Laomedea angulata & Mediterranean & $\mathrm{R}$ & $\mathrm{P}$ & Aliani \& Molcard 2003 \\
\hline Laomedea calceolifera & Northwest Europe & $\mathrm{C}$ & n.i. & Cornelius 1992a \\
\hline Laomedea flexиоsa & Northwest Europe & $\mathrm{C}$ & n.i. & Cornelius 1992a \\
\hline Laomedea sp. & $\begin{array}{l}\text { Northwest Atlantic, } \\
\text { Sargasso Sea }\end{array}$ & $\mathrm{R}$ & M & $\begin{array}{l}\text { Adams 1960, Coston-Clements et al. } \\
1991\end{array}$ \\
\hline Liriope tetraphylla & Sagami Bay, Japan & $\mathrm{R}$ & M & Hirosaki 1964 \\
\hline Monotheca margaretta & Bermuda & $\mathrm{R}$ & M & Adams 1960, Calder 1995 \\
\hline Obelia bicuspidata & Sargasso Sea & $\mathrm{R}$ & M & $\begin{array}{l}\text { Butler et al. 1983, Coston-Clements } \\
\text { et al. } 1991\end{array}$ \\
\hline Obelia bidentata & Northwest Europe & $\mathrm{C}$ & n.i. & Cornelius 1992a \\
\hline Obelia dichotoma & $\begin{array}{l}\text { Mediterranean, } \\
\text { Sargasso Sea, } \\
\text { Bermuda, } \\
\text { Northwest Europe }\end{array}$ & $\mathrm{C}, \mathrm{R}$ & $\mathrm{M}, \mathrm{P}$ & $\begin{array}{l}\text { Butler et al. 1983, Coston-Clements } \\
\text { et al. 1991, Cornelius 1992a, Calder } \\
\text { 1995, Aliani \& Molcard } 2003\end{array}$ \\
\hline Obelia exiqua & North Pacific & $\mathrm{R}$ & $\mathrm{O}$ & Sinitsyn \& Reznichenko 1981 \\
\hline Obelia geniculata & $\begin{array}{l}\text { St. Helena, Sargasso } \\
\text { Sea, Northwest } \\
\text { Europe, Falkland } \\
\text { Current, Japan }\end{array}$ & $\mathrm{C}, \mathrm{R}$ & M & $\begin{array}{l}\text { Hentschel 1922, Arnaud et al. 1976, } \\
\text { Butler et al. 1983, Coston-Clements } \\
\text { et al. 1991, Cornelius 1992a, Sano } \\
\text { et al. } 2003\end{array}$ \\
\hline
\end{tabular}




\section{MARTIN THIEL \& LARS GUTOW}

Table 4 (continued) Cnidaria reported or inferred as rafting

\begin{tabular}{|c|c|c|c|c|}
\hline Species & Region & $\begin{array}{l}\text { Rafting } \\
\text { inference }\end{array}$ & $\begin{array}{l}\text { Floating } \\
\text { substratum }\end{array}$ & Reference \\
\hline Obelia hyalina & Sargasso Sea & $\mathrm{R}$ & M & $\begin{array}{l}\text { Hentschel 1922*, Timmermann } \\
\text { 1932, Butler et al. 1983, } \\
\text { Coston-Clements et al. } 1991\end{array}$ \\
\hline Obelia longissima & $\begin{array}{l}\text { Azores, Northwest } \\
\text { Europe }\end{array}$ & $\mathrm{C}, \mathrm{R}$ & M & $\begin{array}{l}\text { Cornelius 1992a, Morton \& Britton } \\
2000\end{array}$ \\
\hline Obelia plana & Japan & $\mathrm{R}$ & M & Sano et al. 2003 \\
\hline Orthopyxis crenata & Northwest Europe & $\mathrm{D}$ & n.i. & Cornelius 1992a \\
\hline Orthopyxis integra & Northwest Europe & $\mathrm{C}$ & n.i. & Cornelius 1992a \\
\hline Pennaria disticha & Northwest Europe & $\mathrm{D}$ & n.i. & Cornelius 1992a \\
\hline Physalis physalis & Sagami Bay, Japan & $\mathrm{R}$ & M & Hirosaki 1964 \\
\hline Plumularia corrugata & Sargasso Sea & $\mathrm{R}$ & M & $\begin{array}{l}\text { Butler et al. 1983, Coston-Clements } \\
\text { et al. } 1991\end{array}$ \\
\hline Plumularia diaphana & Sargasso Sea & $\mathrm{R}$ & M & $\begin{array}{l}\text { Butler et al. 1983, Coston-Clements } \\
\text { et al. } 1991\end{array}$ \\
\hline Plumularia floridana & Sargasso Sea & $\mathrm{R}$ & M & $\begin{array}{l}\text { Butler et al. 1983, Coston-Clements } \\
\text { et al. } 1991\end{array}$ \\
\hline Plumularia margaretta & $\begin{array}{l}\text { Northwest Atlantic, } \\
\text { Sargasso Sea }\end{array}$ & $\mathrm{R}$ & M & $\begin{array}{l}\text { Adams } 1960^{*}, \text { Butler et al. } 1983 \text {, } \\
\text { Coston-Clements et al. } 1991\end{array}$ \\
\hline $\begin{array}{l}\text { Plumularia } \\
\text { megalocephala }\end{array}$ & Sargasso Sea & $\mathrm{R}$ & M & $\begin{array}{l}\text { Butler et al. 1983, Coston-Clements } \\
\text { et al. } 1991\end{array}$ \\
\hline Plumularia obliqua & $\begin{array}{l}\text { Sargasso Sea, } \\
\text { Northwest } \\
\text { Atlantic, Brazil } \\
\text { Current }\end{array}$ & $\mathrm{R}$ & M & $\begin{array}{l}\text { Hentschel 1922, Adams 1960, } \\
\text { Coston-Clements et al. } 1991\end{array}$ \\
\hline Plumularia sargassi & Sargasso Sea & $\mathrm{R}$ & M & $\begin{array}{l}\text { Hentschel 1922, Coston-Clements } \\
\text { et al. } 1991\end{array}$ \\
\hline Plumularia setacea & Northwest Europe & $\mathrm{D}$ & n.i. & Cornelius 1992a \\
\hline Plumularia setaceoides & Sargasso Sea & $\mathrm{R}$ & M & $\begin{array}{l}\text { Butler et al. 1983, Coston-Clements } \\
\text { et al. } 1991\end{array}$ \\
\hline Plumularia strictocarpa & $\begin{array}{l}\text { Sargasso Sea, } \\
\text { Bermuda }\end{array}$ & $\mathrm{R}$ & M & $\begin{array}{l}\text { Butler et al. 1983, Coston-Clements } \\
\text { et al. 1991, Calder } 1995\end{array}$ \\
\hline Porpita umbella & Sagami Bay, Japan & $\mathrm{R}$ & M & Hirosaki 1964 \\
\hline Sarsia tubulosa & Northwest Europe & $\mathrm{D}$ & n.i. & Cornelius 1992a \\
\hline Scandia mutabilis & Sargasso Sea & $\mathrm{R}$ & M & $\begin{array}{l}\text { Butler et al. 1983, Coston-Clements } \\
\text { et al. } 1991\end{array}$ \\
\hline Sertularella gaudichaudi & Northwest Europe & $\mathrm{D}$ & n.i. & Cornelius 1992a \\
\hline Sertularella miurensis & Japan & $\mathrm{R}$ & M & Sano et al. 2003 \\
\hline Sertularia amplectens & Sargasso Sea & $\mathrm{R}$ & M & $\begin{array}{l}\text { Butler et al. 1983, Coston-Clements } \\
\text { et al. } 1991\end{array}$ \\
\hline Sertularia brevicyathus & Sargasso Sea & $\mathrm{R}$ & M & Coston-Clements et al. 1991 \\
\hline Sertularia cornicina & Sargasso Sea & $\mathrm{R}$ & M & $\begin{array}{l}\text { Butler et al. 1983, Coston-Clements } \\
\text { et al. } 1991\end{array}$ \\
\hline Sertularia exigua & Sargasso Sea & $\mathrm{R}$ & M & $\begin{array}{l}\text { Butler et al. 1983, Coston-Clements } \\
\text { et al. } 1991\end{array}$ \\
\hline Sertularia flowersi & Sargasso Sea & $\mathrm{R}$ & M & $\begin{array}{l}\text { Butler et al. 1983, Coston-Clements } \\
\text { et al. } 1991\end{array}$ \\
\hline Sertularia gracilis & Sargasso Sea & $\mathrm{R}$ & M & $\begin{array}{l}\text { Butler et al. 1983, Coston-Clements } \\
\text { et al. } 1991\end{array}$ \\
\hline
\end{tabular}




\section{RAFTING OF BENTHIC MARINE ORGANISMS}

Table 4 (continued) Cnidaria reported or inferred as rafting

\begin{tabular}{|c|c|c|c|c|}
\hline Species & Region & $\begin{array}{l}\text { Rafting } \\
\text { inference }\end{array}$ & $\begin{array}{l}\text { Floating } \\
\text { substratum }\end{array}$ & Reference \\
\hline Sertularia inflata & Sargasso Sea & $\mathrm{R}$ & M & $\begin{array}{l}\text { Butler et al. 1983, Coston-Clements } \\
\text { et al. } 1991\end{array}$ \\
\hline Sertularia marginata & Sargasso Sea & $\mathrm{R}$ & M & Hentschel $1922 *$ \\
\hline Sertularia mayeri & Sargasso Sea & $\mathrm{R}$ & M & $\begin{array}{l}\text { Hentschel 1922, Timmermann } 1932, \\
\text { Butler et al. 1983, Coston-Clements } \\
\text { et al. } 1991\end{array}$ \\
\hline Sertularia pumila & n.i. & $\mathrm{R}$ & M & Vallentin 1895 \\
\hline Sertularia sp. & California & $\mathrm{R}$ & $\mathrm{O}$ & Worcester 1994 \\
\hline Sertularia stookeyi & Sargasso Sea & $\mathrm{R}$ & M & $\begin{array}{l}\text { Butler et al. 1983, Coston-Clements } \\
\text { et al. } 1991\end{array}$ \\
\hline Sertularia turbinata & $\begin{array}{l}\text { Sargasso Sea, North } \\
\text { Pacific }\end{array}$ & $\mathrm{R}$ & $\mathrm{M}, \mathrm{O}$ & $\begin{array}{l}\text { Reznichenko 1981, Sinitsyn \& } \\
\text { Reznichenko 1981, Butler et al. } \\
\text { 1983, Coston-Clements et al. 1991, } \\
\text { Tsikhon-Lukanina et al. 2001a }\end{array}$ \\
\hline Sertularia versluysi & Sargasso Sea & $\mathrm{R}$ & $\mathrm{M}$ & Coston-Clements et al. 1991 \\
\hline Stereotheca elongata & Northwest Europe & $\mathrm{D}$ & n.i. & $\begin{array}{l}\text { Ritchie 1908, cited in Cornelius } \\
\text { 1992a }\end{array}$ \\
\hline Syncoryne mirabilis & Sargasso Sea & $\mathrm{R}$ & M & $\begin{array}{l}\text { Butler et al. 1983, Coston-Clements } \\
\text { et al. } 1991\end{array}$ \\
\hline Tridentata distans & Northwest Europe & $\mathrm{D}$ & n.i. & Cornelius 1992a \\
\hline Tubularia crocea & Northwest Europe & $\mathrm{D}$ & n.i. & Cornelius 1992a \\
\hline Ventromma halecioides & Northwest Europe & $\mathrm{C}$ & n.i. & Cornelius 1992a \\
\hline Zanclea costata & $\begin{array}{l}\text { Northwest Atlantic, } \\
\text { Sargasso Sea }\end{array}$ & $\mathrm{R}$ & $\mathrm{M}$ & $\begin{array}{l}\text { Adams } 1960^{*} \text {, Butler et al. } 1983 \text {, } \\
\text { Coston-Clements et al. } 1991\end{array}$ \\
\hline Zanclea gemmosa & Sargasso Sea & $\mathrm{R}$ & M & $\begin{array}{l}\text { Butler et al. 1983, Coston-Clements } \\
\text { et al. } 1991\end{array}$ \\
\hline Zanclea implexa & Sargasso Sea & $\mathrm{R}$ & M & Hentschel $1922 *$ \\
\hline \multicolumn{5}{|l|}{ Anthozoa } \\
\hline Acropora sp. & Fiji to Australia & $\mathrm{R}$ & $\mathrm{V}$ & Jokiel 1990a \\
\hline Anemonia sargassensis & $\begin{array}{l}\text { Northwest Atlantic, } \\
\text { Sargasso Sea }\end{array}$ & $\mathrm{R}$ & M & $\begin{array}{l}\text { Adams 1960, Fine 1970, Butler et al. } \\
\text { 1983, Coston-Clements et al. } 1991\end{array}$ \\
\hline Anemonia sulcata & Northwest Europe & $\mathrm{R}$ & M & Vallentin $1895^{*}$ \\
\hline Anthopleura spp. & California & $\mathrm{R}$ & M & Bushing 1994 \\
\hline Balanophyllia elegans & California & $\mathrm{D}, \mathrm{R}$ & M & Gerrodette 1981, Bushing 1994 \\
\hline Boloceroides mcmurrichi & Sagami Bay, Japan & $\mathrm{R}$ & $\mathrm{O}$ & Hirosaki 1964 \\
\hline Corynactis californica & California & $\mathrm{R}$ & M & Bushing 1994 \\
\hline Cricophorus nutrix & Chatham Islands & $\mathrm{D}$ & M & Knox 1954 \\
\hline Cyphastrea sp. & Fiji to Australia & $\mathrm{R}$ & $\mathrm{V}$ & Jokiel 1990a \\
\hline Epiactis fernaldi & Washington & G & $\mathrm{O}, \mathrm{W}$ & Edmands \& Potts 1997 \\
\hline Epiactis lisbethae & Washington & G & $\mathrm{O}, \mathrm{W}$ & Edmands \& Potts 1997 \\
\hline Epiactis prolifera & $\begin{array}{l}\text { California, } \\
\text { Washington }\end{array}$ & $\mathrm{G}, \mathrm{R}$ & $\begin{array}{l}\mathrm{M}, \mathrm{O}, \\
\mathrm{W}\end{array}$ & $\begin{array}{l}\text { Bushing 1994, Edmands \& Potts } \\
\text { 1997, Hobday 2000a }\end{array}$ \\
\hline Epiactis ritteri & Washington & $\mathrm{G}$ & $\mathrm{O}, \mathrm{W}$ & Edmands \& Potts 1997 \\
\hline Favia sp. & Fiji to Australia & $\mathrm{R}$ & $\mathrm{V}$ & Jokiel 1990a \\
\hline Goniopora sp. & Fiji to Australia & $\mathrm{R}$ & $\mathrm{V}$ & Jokiel 1990a \\
\hline Millepora sp. & Marshall Islands & B & $\mathrm{V}$ & Jokiel 1989 \\
\hline Millepora sp. & Florida & B & $P$ & Winston et al. 1997 \\
\hline
\end{tabular}


MARTIN THIEL \& LARS GUTOW

Table 4 (continued) Cnidaria reported or inferred as rafting

\begin{tabular}{|c|c|c|c|c|}
\hline Species & Region & $\begin{array}{l}\text { Rafting } \\
\text { inference }\end{array}$ & $\begin{array}{l}\text { Floating } \\
\text { substratum }\end{array}$ & Reference \\
\hline Montipora sp. & Fiji to Australia & $\mathrm{R}$ & $\mathrm{V}$ & Jokiel 1990a \\
\hline Phyllangia americana & Florida & B & $\mathrm{P}$ & Winston et al. 1997 \\
\hline Pocillopora damicornis & Hawaii & B & $\mathrm{S}, \mathrm{W}$ & $\begin{array}{l}\text { Crossland 1952, cited in Richmond } \\
1987\end{array}$ \\
\hline Pocillopora sp. & Hawaii & $\mathrm{R}$ & $\mathrm{P}, \mathrm{V}$ & Jokiel 1984 \\
\hline Pocillopora sp. & Fiji to Australia & $\mathrm{R}$ & $\mathrm{V}$ & Jokiel 1990a \\
\hline Pocillopora spp. & Marshall Islands & B & $\mathrm{V}$ & Jokiel 1989 \\
\hline Pocillopora verrucosa & Lizard Island & B & $\mathrm{W}$ & Jokiel 1989 \\
\hline Porites lutea & Marshall Islands & $\mathrm{B}$ & V & Jokiel 1989 \\
\hline Porites sp. & Fiji to Australia & $\mathrm{R}$ & $\mathrm{V}$ & Jokiel 1990a \\
\hline Seriatopora sp. & Fiji to Australia & $\mathrm{R}$ & $\mathrm{V}$ & Jokiel 1990a \\
\hline Stylophora sp. & Fiji to Australia & $\mathrm{R}$ & V & Jokiel 1990a \\
\hline \multicolumn{5}{|l|}{ Scyphozoa } \\
\hline Aurelia aurita & Sagami Bay, Japan & $\mathrm{R}$ & M & Hirosaki 1964 \\
\hline
\end{tabular}

Notes: $\mathrm{B}=$ stranded floating item on beach $; \mathrm{C}=$ circumstantial $; \mathrm{D}=$ distributional inference; $\mathrm{G}=$ genetic inference; $\mathrm{M}=$ macroalgae; n.i. = no information; $\mathrm{O}=$ other; $\mathrm{P}=$ plastics; $\mathrm{R}=$ rafting; $\mathrm{S}=$ seeds; $\mathrm{V}=$ volcanic pumice; $\mathrm{W}=$ wood.

* References refer to this species under a different name.

different species from floating Sargassum collected in the Sargasso Sea. He remarked that the species found on Sargassum are comparatively small and he suggested that this might be due to the limited availability of food in the oligotrophic Sargasso Sea. Niermann (1986), observing lower abundances of hydroids Clytia noliformis (and of bryozoans) on Sargassum spp. south of the thermal front, i.e., in the Sargasso Sea proper, also suggested that this might be due to food limitations of these species, which feed on suspended microalgae. This agrees with the findings of Smith et al. (1973) that respiration of the entire Sargassum community was higher in the nutrient-rich continental Slope Water than in the oligotrophic Sargasso Sea.

Calder (1995) found a total of 10 hydroid species on floating Sargassum collected near Bermuda. In accordance with Smith et al. (1973) he found that species of the genus Clytia and Aglaophenia were among the numerically and functionally most important animals on floating Sargassum in that region. The two algal species $S$. natans and $S$. fluitans differed from each other in the composition and community structure of associated hydroids. Gonophores were present in different stages of development indicating that the hydroids reproduce and produce medusae while attached to floating algae. The hydroid fauna of the Azores, however, is dominated by species without medusae, which together with frequent observations on floating items, led Cornelius (1992a) to conclude that most species had reached the oceanic islands of the Azores via rafting. He reported hydroids from macroalgae and floating plastics.

From floating objects in the Ligurian Sea (western Mediterranean) consisting mainly of anthropogenic debris but also of natural floats such as wood and leaves, Aliani \& Molcard (2003) recorded five different hydroid species. The Hydrozoa ranked among the most diverse taxa with more than $20 \%$ of the total number of species found on floating items in that region. Arnaud et al. (1976) found some colonies of a single hydroid species on floating kelp (most probably Ecklonia maxima) off the island of St. Helena. Gregory (1978) even reported hydroids growing on very small substrata such as plastic granules collected on beaches of New Zealand (see also Carpenter \& Smith 1972). 


\section{RAFTING OF BENTHIC MARINE ORGANISMS}

\section{Cnidaria (Anthozoa)}

Sea anemones and corals have been reported from different floating substrata including macroalgae, volcanic pumice and plastics (Jokiel 1984, 1990a, Hobday 2000a) (Table 4). The sea anemone Anemonia alicemartinae can be frequently found on floating macroalgae off the Pacific coast of Chile (M. Thiel, personal observation). Individuals of the sea anemone Epiactis prolifera with brooded offspring were collected on floating macroalgae Macrocystis pyrifera (Bushing 1994). The same author also reported the solitary scleractinian coral Balanophyllia elegans. Demersal planula larvae of this species crawl over the benthic substratum at a speed of $1-4 \mathrm{~mm} \mathrm{~min}{ }^{-1}$ (Gerrodette 1981), which cannot explain the widespread distribution of this species. The author suggested that rafting of adults on stones held by the haptera of detached kelps might be a possible dispersal mechanism for this species. However, rafting of Balanophyllia elegans has not yet been observed directly. Ottaway \& Kirby (1975) suggested asexual reproduction or apomictic parthenogenesis for the brooding sea anemone Actinia tenebrosa, which might be a useful trait of a rafting organism upon reaching new habitats (see below).

Corals may float themselves, but usually it is only the air-filled skeletons of dead specimens that are able to float (Kornicker \& Squires 1962). Live corals found growing on volcanic pumice have apparently rafted over large distances as was suggested by colony sizes (Jokiel 1984, 1990a). Coral colonies of Pocillopora sp. growing on floating pumice at Hawaii were reproductive and had sizes that even indicated an age of 2-3 yr (Jokiel 1984). During this time the colonies could have covered a distance of about $20,000-40,000 \mathrm{~km}$, corresponding to several circuits around the tropical and subtropical Pacific basin (Jokiel 1984). Common reports of stranded glass buoys lost by Japanese fishermen that were covered by corals led the author to the conclusion that some corals colonising Hawaii have their origin in the Western Pacific. Floating pumice, however, most likely came from San Benedicto (Mexico) indicating that the Hawaiian archipelago is colonised from several sources. Furthermore, $P$. damicornis has been found attached to coconuts and logs (Crossland 1952 cited in Richmond 1987). Richmond (1987), however, doubts that $P$. damicornis colonised the eastern Pacific region by rafting. In a personal communication to Richmond (1987) G.M. Wellington reported on histological observations he made on $P$. damicornis from that region: even though he observed gonadal development, the animals never reached full sexual maturity. Thus, larval recruits that are encountered in that region must have arrived there as planktonic larvae (see also Scheltema 1988, Glynn \& Ault 2000). In addition to sexual reproduction, self-fertilisation and asexual production of planula larvae has been described for corals from this genus (Stoddart 1983), making them highly suited to colonise new habitats after arrival on a floating item.

\section{Ctenophora}

Bushing (1994) collected a single undetermined ctenophore with floating macroalgae. Since ctenophores are typically pelagic it remains questionable whether these animals can be classified as rafters. Davenport \& Rees (1993) analysed the stomach content of ctenophores (Pleurobrachia pileus) in the Irish Sea without mentioning whether these animals were associated with floating algae. They revealed that portunid larvae are an important component of the diet of ctenophores. Larvae and megalopae of swimming crabs are quite abundant in floating weed patches from that region. Similar to other pelagic predators, ctenophores might at least loosely (or passively) associate with floating algae where food is easily available.

\section{Platyhelminthes}

Turbellarians were mentioned as abundant epifauna on floating items in the Northwest Pacific (Tsikhon-Lukanina et al. 2001a) (Table 5). Platyhelminthes are also found quite frequently on 


\section{MARTIN THIEL \& LARS GUTOW}

Table 5 Platyhelminthes, Nemertea and Nematoda reported or inferred as rafting

\begin{tabular}{|c|c|c|c|c|}
\hline Species & Region & $\begin{array}{l}\text { Rafting } \\
\text { inference }\end{array}$ & $\begin{array}{l}\text { Floating } \\
\text { substratum }\end{array}$ & Reference \\
\hline \multicolumn{5}{|l|}{ Platyhelminthes } \\
\hline Acerotisa notulata & $\begin{array}{l}\text { Sargasso Sea, North } \\
\text { Atlantic }\end{array}$ & $\mathrm{R}$ & M & $\begin{array}{l}\text { Hyman 1939, Butler et al. 1983, } \\
\text { Coston-Clements et al. } 1991\end{array}$ \\
\hline Acerotisa sp. & Sargasso Sea & $\mathrm{R}$ & M & Fine 1970 \\
\hline $\begin{array}{l}\text { Amphiscolops } \\
\text { bermudensis }\end{array}$ & Bermuda & $\mathrm{R}$ & M & Hyman 1939 \\
\hline Amphiscolops sargassi & Sargasso Sea & $\mathrm{R}$ & M & $\begin{array}{l}\text { Butler et al. 1983, Coston-Clements } \\
\text { et al. } 1991\end{array}$ \\
\hline $\begin{array}{l}\text { Gnescioceros } \\
\text { sargassicola }\end{array}$ & $\begin{array}{l}\text { Sargasso Sea, Gulf } \\
\text { Stream, Northwest } \\
\text { Atlantic }\end{array}$ & $\mathrm{B}, \mathrm{R}$ & M & $\begin{array}{c}\text { Hyman 1939, Adams 1960*, Fine 1970, } \\
\text { Butler et al. 1983, Stoner \& Greening } \\
\text { 1984, Coston-Clements et al. 1991* }\end{array}$ \\
\hline Hoploplana grubei & $\begin{array}{l}\text { North Atlantic, Gulf of } \\
\text { Mexico, Sargasso Sea, } \\
\text { Gulf Stream }\end{array}$ & $\mathrm{R}$ & M & $\begin{array}{l}\text { Hyman 1939, Fine 1970, Butler et al. } \\
\text { 1983, Stoner \& Greening 1984, } \\
\text { Coston-Clements et al. } 1991\end{array}$ \\
\hline Leptoplana sp. & Red Sea & $\mathrm{R}$ & M & Markkaveeva 1965 \\
\hline Planocera pellucida & $\begin{array}{l}\text { Northwest Atlantic, } \\
\text { Sargasso Sea, Kuroshio }\end{array}$ & $\mathrm{R}$ & M & $\begin{array}{l}\text { Adams } 1960^{*} \text {, Safran \& Omori } 1990 \text {, } \\
\text { Coston-Clements et al. 1991* }\end{array}$ \\
\hline $\begin{array}{l}\text { Prosthiostomum } \\
\text { siphunculus }\end{array}$ & Tasmania & $\mathrm{R}$ & M & Edgar 1987 \\
\hline Proshiostomum sp. & Red Sea & $\mathrm{R}$ & M & Markkaveeva 1965 \\
\hline $\begin{array}{l}\text { Stylochoplana } \\
\text { sargassicola }\end{array}$ & Baja California & $\mathrm{R}$ & M & Cheng \& Lewin 1975 \\
\hline \multicolumn{5}{|l|}{ Nemertea } \\
\hline Tetrastemma candidum & Northwest coast of U.S. & $\mathrm{R}$ & M & Highsmith 1985 \\
\hline Zygonemertes virescens & Northwest coast of U.S. & $\mathrm{R}$ & M & Highsmith 1985 \\
\hline \multicolumn{5}{|l|}{ Nematoda } \\
\hline Anticoma sp. & Bermuda & B & $\mathrm{S}$ & Gerlach 1977 \\
\hline Chromadora filiformis & Sargasso Sea & $\mathrm{R}$ & M & Micoletzky 1922 \\
\hline $\begin{array}{l}\text { Chromadora } \\
\text { nudicapitata }\end{array}$ & Sargasso Sea & $\mathrm{R}$ & M & Micoletzky 1922 \\
\hline Cyatholaimus sp. & Bermuda & B & $\mathrm{S}$ & Gerlach 1977 \\
\hline Euchromadora sp. & Bermuda & B & $\mathrm{S}$ & Gerlach 1977 \\
\hline $\begin{array}{l}\text { Leptosomatum } \\
\text { elongatum }\end{array}$ & Tortuge (Venezuela) & $\mathrm{R}$ & M & Steiner 1915 cited in Micoletzky 1922 \\
\hline Oncholaimus dujardini & Bermuda & B & S & Gerlach 1977 \\
\hline Phanoderma sp. & Bermuda & B & S & Gerlach 1977 \\
\hline Spilophorella paradoxa & Bermuda & B & S & Gerlach 1977 \\
\hline Synonema braziliense & Bermuda & B & S & Gerlach 1977 \\
\hline $\begin{array}{l}\text { Syringolaimus } \\
\text { striatocaudatus }\end{array}$ & Bermuda & B & S & Gerlach 1977 \\
\hline Viscosia sp. & Bermuda & B & $\mathrm{S}$ & Gerlach 1977 \\
\hline
\end{tabular}

Notes: $\mathrm{B}=$ stranded floating item on beach; $\mathrm{M}=$ macroalgae; $\mathrm{R}=$ rafting; $\mathrm{S}=$ seeds.

* References refer to this species under a different name. 


\section{RAFTING OF BENTHIC MARINE ORGANISMS}

floating macroalgae (Bushing 1994). Hyman (1939) identified several turbellarian species from floating Sargassum. The species Gnesioceros sargassicola is a very common polyclad on floating Sargassum (Hyman 1939) in the Gulf of Mexico, the Caribbean and the Sargasso Sea and can thus be expected to be able to spend its entire life cycle on floating algae. Hyman (1939) noted the taxonomic affinity of this rafting species with Gnesioceros sargassicola var. lata from attached Sargassum spp. at Bermuda. She also remarked on the small body size of the species Acerotisa notulata from floating Sargassum spp. (Hyman 1939).

\section{Nemertea}

Small numbers of nemerteans were found on floating macroalgae off southern California (Bushing 1994) (Table 5). Around Iceland, nemerteans were rare on floating macroalgae except on rafts collected at a distance of about $10-15 \mathrm{~km}$ from the coast on which an average of more than 200 individuals per $\mathrm{kg}$ algal wet weight were found (Ingólfsson 1995).

\section{Nemathelminthes (Nematoda)}

Gerlach (1977) listed several meiofaunal nematode species (together with animals from other taxa) that were washed out from the fibrous surface of a floating coconut found off Bermuda (Table 5). He suggested that the coconut might have become colonised by the animals when it was rolling in the beach surf. Many of the nematodes from the coconut belonged to species with a cosmopolitan distribution. Furthermore, nematodes inhabit floating Sargassum spp. in the Atlantic (Gerlach 1977). Micoletzky (1922) found the most common benthic nematode species of the Atlantic region on floating Sargassum. He considered highly efficient caudal glands that allow the animals to fix themselves to the algal substratum and viviparity or the ability to glue eggs to the algal surface, respectively, as the most important preconditions of nematodes for the successful colonisation of floating algae. An extensive comparison of animals from floating Sargassum and floating coal slag with conspecifics from attached Sargassum in the Mediterranean and the Red Sea revealed that rafting individuals are slightly smaller than benthic conspecifics (Micoletzky 1922). Egg-carrying females proved to be reproductively active during rafting. However, the proportion of ovigerous females was only $30-50 \%$ of that observed in benthic populations.

Colonisation of floating objects by usually benthic meiofauna species such as nematodes could be the result of an accidental encounter with the raft while the animals are temporarily suspended in the water column. Gerlach (1977) reported benthic nematodes that were captured in plankton tows and in sediment traps above the sea bed. In petri dishes he observed wriggling movements of nematodes different from normal locomotion and he questioned whether these movements might serve for swimming in the pelagial. Walters \& Bell (1986), too, found nematodes in the water column over shallow-water seagrass beds in Tampa Bay, Florida. In her review on dispersal of marine meiofauna, Palmer (1988) described that meiofaunal species enter the water column either actively or passively. Active migration often appears to be linked to low current velocities (tidal rhythm). Passive suspension of meiofauna species results from turbulences generated by waves or currents. Nematodes suspended in the water column may then subsequently attach to floating items.

Nematodes appear to be among the dominant rafting organisms in the matrix of floating detritus (Phillips 1963, Faust \& Gulledge 1996). With a proportion of about 5\%, nematodes represented the second-most abundant group on floating detritus patches sampled in shallow tidal channels in New Zealand (Hicks 1988).

\section{Annelida}

Polychaetes (Sedentaria) are common and often abundant members of the rafting fauna (Barnes 2002) (Table 6). They occur on macroalgae (Butler et al. 1983, Edgar 1987, Hobday 2000a) and 


\section{MARTIN THIEL \& LARS GUTOW}

Table 6 Annelida and Tardigrada reported or inferred as rafting

\begin{tabular}{|c|c|c|c|c|}
\hline Species & Region & $\begin{array}{l}\text { Rafting } \\
\text { inference }\end{array}$ & $\begin{array}{l}\text { Floating } \\
\text { substratum }\end{array}$ & Reference \\
\hline \multicolumn{5}{|l|}{ Polychaeta } \\
\hline Amphinome rostrata & $\begin{array}{l}\text { Sargasso Sea, North } \\
\text { Pacific }\end{array}$ & $\mathrm{R}$ & $\mathrm{M}, \mathrm{O}$ & $\begin{array}{l}\text { Sinitsyn \& Reznichenko 1981, } \\
\text { Butler et al. 1983, } \\
\text { Coston-Clements et al. 1991, } \\
\text { Salazar-Vallejo } 1992\end{array}$ \\
\hline Amphinome vagans & Panama & $\mathrm{R}$ & $\mathrm{W}$ & Donlan \& Nelson 2003 \\
\hline Autolytus simplex & Subantarctica & $\mathrm{R}$ & M & Averincev 1980 \\
\hline Autolytus sp. & Iceland & $\mathrm{R}$ & M & Ingólfsson 1995 \\
\hline Brana rhopallophora & Subantarctica & $\mathrm{R}$ & M & Averincev 1980 \\
\hline Circeis armoricana & California & $\mathrm{B}$ & M & Knight-Jones 1978 \\
\hline Cirratulus fulgida & Subantarctica & $\mathrm{R}$ & M & Averincev 1980 \\
\hline Disponetus bidentatus & Subantarctica & $\mathrm{R}$ & M & Averincev 1980 \\
\hline Eteone sculpta & Subantarctica & $\mathrm{R}$ & M & Averincev 1980 \\
\hline Eunice kobiensis & North Pacific & $\mathrm{R}$ & $\mathrm{O}$ & Sinitsyn \& Reznichenko 1981 \\
\hline Euphrosyne orientalis & North Pacific & $\mathrm{R}$ & $\mathrm{O}$ & Sinitsyn \& Reznichenko 1981 \\
\hline $\begin{array}{l}\text { Eupolymnia } \\
\text { heterobranchia }\end{array}$ & Northwest coast of U.S. & $\mathrm{R}$ & M & Highsmith 1985 \\
\hline Exogone heterosetosa & Subantarctica & $\mathrm{R}$ & M & Averincev 1980 \\
\hline Exogone lourei & Northwest coast of U.S. & $\mathrm{R}$ & M & Highsmith 1985 \\
\hline Exogone sp. & Tasmania & $\mathrm{R}$ & M & Edgar 1987 \\
\hline Fabricia sabella & Iceland & $\mathrm{R}$ & M & Ingólfsson 1995, 1998 \\
\hline Genetyllus polyphylla & Subantarctica & $\mathrm{R}$ & M & Averincev 1980 \\
\hline Harmothoe dearborni & Sargasso Sea & $\mathrm{R}$ & M & $\begin{array}{l}\text { Butler et al. 1983, Coston- } \\
\text { Clements et al. } 1991\end{array}$ \\
\hline Harmothoe imbricata & Northwest coast of U.S. & $\mathrm{R}$ & M & Highsmith 1985 \\
\hline Harmothoe impar & North Sea & $\mathrm{R}$ & W & Michaelsen 1897 \\
\hline Harmothoe spinosa & Subantarctica & $\mathrm{R}$ & M & Averincev 1980 \\
\hline Harmothoe sp. & Tasmania & $\mathrm{R}$ & M & Edgar 1987 \\
\hline Harmothoe sp. & Iceland & $\mathrm{R}$ & M & Ingólfsson 1995 \\
\hline Hediste japonica & Sagami Bay, Japan & $\mathrm{R}$ & M & Hirosaki 1964 \\
\hline Helicosiphon platyspira & Marion Island & $\mathrm{D}$ & n.i. & Knight-Jones 1978 \\
\hline Hipponoe gaudichaudi & North Pacific & $\mathrm{R}$ & $\mathrm{O}$ & $\begin{array}{l}\text { Sinitsyn \& Reznichenko 1981, } \\
\text { Salazar-Vallejo } 1992\end{array}$ \\
\hline Hydroides dianthus & Florida & B & $\mathrm{P}$ & Winston et al. 1997 \\
\hline Hydroides elegans & New Zealand & B & $\mathrm{P}$ & Winston et al. 1997 \\
\hline Hydroides sp. & Sagami Bay, Japan & $\mathrm{R}$ & M & Hirosaki 1964 \\
\hline Janua brasiliensis & Great Britain & $\mathrm{D}$ & M & Knight-Jones et al. 1975 \\
\hline Janua formosa & Sargasso Sea & $\mathrm{R}$ & M & Ryland 1974 \\
\hline $\begin{array}{l}\text { Lumbrinereis } \\
\text { magalhaensis }\end{array}$ & Subantarctica & $\mathrm{R}$ & M & Averincev 1980 \\
\hline Myriochele sp. & Iceland & $\mathrm{R}$ & M & Ingólfsson 1995 \\
\hline Naiades cantrainii & $\begin{array}{l}\text { Sargasso Sea, Northwest } \\
\text { Atlantic }\end{array}$ & $\mathrm{R}$ & M & $\begin{array}{l}\text { Adams } 1960^{*}, \text { Coston- } \\
\text { Clements et al. } 1991^{*}\end{array}$ \\
\hline Neanthes kerguelensis & Subantarctica & $\mathrm{R}$ & M & Averincev 1980 \\
\hline Neodexispira brasiliensis & Southern oceans & $\mathrm{D}$ & M & $\begin{array}{l}\text { Knight-Jones \& Knight-Jones } \\
1984\end{array}$ \\
\hline Nereis falsa & Mediterranean & $\mathrm{R}$ & $\mathrm{P}$ & Aliani \& Molcard 2003 \\
\hline Nereis maxillodentata & Tasmania & $\mathrm{R}$ & M & Edgar 1987 \\
\hline Nereis pelagica & Sagami Bay, Japan & $\mathrm{R}$ & M & Hirosaki 1964 \\
\hline
\end{tabular}




\section{RAFTING OF BENTHIC MARINE ORGANISMS}

Table 6 (continued) Annelida and Tardigrada reported or inferred as rafting

\begin{tabular}{|c|c|c|c|c|}
\hline Species & Region & $\begin{array}{l}\text { Rafting } \\
\text { inference }\end{array}$ & $\begin{array}{l}\text { Floating } \\
\text { substratum }\end{array}$ & Reference \\
\hline Ophelia sp. & Tasmania & $\mathrm{R}$ & M & Edgar 1987 \\
\hline Ophiodromus pugettensis & Northwest coast of U.S. & $\mathrm{R}$ & M & Highsmith 1985 \\
\hline Ophryotrocha claparedii & Subantarctica & $\mathrm{R}$ & M & Averincev 1980 \\
\hline Paralaeospira levinseni & Southern oceans & $\mathrm{D}$ & M & $\begin{array}{l}\text { Knight-Jones \& Knight-Jones } \\
1984\end{array}$ \\
\hline Perinereis capensis & St. Helena & $\mathrm{R}$ & M & Arnaud et al. 1976 \\
\hline Pherusa kerguelensis & Subantarctica & $\mathrm{R}$ & M & Averincev 1980 \\
\hline Pholoe antipoda & Subantarctica & $\mathrm{R}$ & M & Averincev 1980 \\
\hline Pileolaria marginata & Baja California & B & M & Knight-Jones 1978 \\
\hline Pionosyllis sp. & Tasmania & $\mathrm{R}$ & M & Edgar 1987 \\
\hline Platynereis agassizi & Sagami Bay, Japan & $\mathrm{R}$ & M & Hirosaki 1964 \\
\hline Platynereis australis & North Pacific & $\mathrm{R}$ & $\mathrm{O}$ & Sinitsyn \& Reznichenko 1981 \\
\hline $\begin{array}{l}\text { Platynereis } \\
\text { bicanaliculata }\end{array}$ & $\begin{array}{l}\text { North Pacific, Northwest } \\
\text { coast of U.S., Japan }\end{array}$ & $\mathrm{R}$ & $\mathrm{M}, \mathrm{O}$ & $\begin{array}{l}\text { Sinitsyn \& Reznichenko 1981, } \\
\text { Highsmith 1985, Sano et al. } \\
2003\end{array}$ \\
\hline Platynereis coccinea & Sargasso Sea & $\mathrm{R}$ & M & $\begin{array}{l}\text { Butler et al. 1983, Coston- } \\
\text { Clements et al. } 1991\end{array}$ \\
\hline Platynereis dumerilii & $\begin{array}{l}\text { Sargasso Sea, Red Sea, } \\
\text { North Pacific, Gulf } \\
\text { Stream }\end{array}$ & $\mathrm{R}$ & $\mathrm{M}, \mathrm{O}$ & $\begin{array}{l}\text { Hentschel 1922*, } \\
\text { Markkaveeva 1965, } \\
\text { Fine 1970, Sinitsyn \& } \\
\text { Reznichenko 1981, Butler } \\
\text { et al. 1983, Stoner \& } \\
\text { Greening 1984, Keller 1987, } \\
\text { Coston-Clements et al. } 1991\end{array}$ \\
\hline Polycirrus insignis & Subantarctica & $\mathrm{R}$ & M & Averincev 1980 \\
\hline Potamilla antarctica & Subantarctica & $\mathrm{R}$ & M & Averincev 1980 \\
\hline Potamilla myriops & North Pacific & $\mathrm{R}$ & $\mathrm{O}$ & $\begin{array}{l}\text { Reznichenko } 1981 \text {, Sinitsyn \& } \\
\text { Reznichenko } 1981\end{array}$ \\
\hline Prionospio cirrifera & Iceland & $\mathrm{R}$ & M & Ingólfsson 1995 \\
\hline Protolaeospira tricostalis & Southern oceans & $\mathrm{D}$ & M & $\begin{array}{l}\text { Knight-Jones \& Knight-Jones } \\
1984\end{array}$ \\
\hline Romanchella perrieri & Southern oceans & $\mathrm{D}$ & M & $\begin{array}{l}\text { Knight-Jones \& Knight-Jones } \\
1984\end{array}$ \\
\hline Romanchella scoresbyi & Southern oceans & $\mathrm{D}$ & M & $\begin{array}{l}\text { Knight-Jones \& Knight-Jones } \\
1984\end{array}$ \\
\hline Scoloplos marginatus & Subantarctica & $\mathrm{R}$ & M & Averincev 1980 \\
\hline Serpula sp. & Thailand & $\mathrm{R}$ & $\mathrm{O}$ & Hamada 1964 \\
\hline Spirobranchus polytrema & Mediterranean & $\mathrm{R}$ & $\mathrm{P}$ & Aliani \& Molcard 2003 \\
\hline Spirorbis alveolatus & North Pacific & $\mathrm{R}$ & $\mathrm{O}$ & $\begin{array}{l}\text { Sinitsyn \& Reznichenko 1981, } \\
\text { Tsikhon-Lukanina et al. } \\
\text { 2001a }\end{array}$ \\
\hline Spirorbis borealis & Iceland, Florida & $\mathrm{B}, \mathrm{R}$ & $\mathrm{O}, \mathrm{P}$ & $\begin{array}{l}\text { Ingólfsson 1995, Winston et al. } \\
1997\end{array}$ \\
\hline Spirorbis corrugatus & Sargasso Sea, Florida & B & $\mathrm{M}, \mathrm{P}$ & $\begin{array}{l}\text { Hentschel 1922, Timmermann } \\
\text { 1932, Scheltema 1977, } \\
\text { Butler et al. 1983*, } \\
\text { Coston-Clements et al. 1991, } \\
\text { Winston et al. } 1997\end{array}$ \\
\hline Spirorbis rothlisbergi & Baja California & B & M & Knight-Jones 1978 \\
\hline
\end{tabular}




\section{MARTIN THIEL \& LARS GUTOW}

Table 6 (continued) Annelida and Tardigrada reported or inferred as rafting

\begin{tabular}{|c|c|c|c|c|}
\hline Species & Region & $\begin{array}{l}\text { Rafting } \\
\text { inference }\end{array}$ & $\begin{array}{l}\text { Floating } \\
\text { substratum }\end{array}$ & Reference \\
\hline Spirorbis rupestris & United Kingdom & $\mathrm{C}$ & $\mathrm{M}$ & Gee 1963 \\
\hline Spirorbis spatulatus & Baja California & B & M & Knight-Jones 1978 \\
\hline Spirorbis spirillum & Florida & B & $\mathrm{P}$ & Winston et al. 1997 \\
\hline Spirorbis sp. & Northwest Atlantic & $\mathrm{R}$ & M & Adams 1960 \\
\hline Spirorbis sp. & Thailand & $\mathrm{R}$ & $\mathrm{O}$ & Hamada 1964 \\
\hline Spirorbis sp. & Sagami Bay, Japan & $\mathrm{R}$ & M & Hirosaki 1964 \\
\hline Spirorbis sp. & Sargasso Sea & $\mathrm{R}$ & M & Keller 1987 \\
\hline $\begin{array}{l}\text { Steggoa magalhaensis } \\
\text { minuta }\end{array}$ & Subantarctica & $\mathrm{R}$ & M & Averincev 1980 \\
\hline Thelepus setosus & Sagami Bay, Japan & $\mathrm{R}$ & M & Hirosaki 1964 \\
\hline Trypanosyllus gigantica & Subantarctica & $\mathrm{R}$ & M & Averincev 1980 \\
\hline Typosyllis aciculata & North Pacific & $\mathrm{R}$ & $\mathrm{O}$ & Sinitsyn \& Reznichenko 1981 \\
\hline Typosyllis sp. & Tasmania & $\mathrm{R}$ & M & Edgar 1987 \\
\hline \multicolumn{5}{|l|}{ Oligochaeta } \\
\hline Oligochaeta sp. 1 & Heard Island (Kerguelen) & $\mathrm{R}$ & M & Edgar \& Burton 2000 \\
\hline Oligochaeta sp. 2 & Iceland & $\mathrm{R}$ & $\mathrm{M}$ & Ingólfsson 1998 \\
\hline \multicolumn{5}{|l|}{ Tardigrada } \\
\hline Styraconyx sargassi & Sargasso Sea & $\mathrm{R}$ & M & $\begin{array}{l}\text { Thulin 1942, Butler et al. } \\
\text { 1983, Coston-Clements et al. } \\
1991\end{array}$ \\
\hline
\end{tabular}

Notes: $\mathrm{B}=$ stranded floating item on beach; $\mathrm{C}=$ circumstantial; $\mathrm{D}=$ distributional inference; $\mathrm{M}=$ macroalgae; $\mathrm{n} . \mathrm{i} .=$ no information; $\mathrm{O}=$ other; $\mathrm{P}=$ plastics; $\mathrm{R}=$ rafting; $\mathrm{W}=$ wood.

* References refer to this species under a different name.

also on plastics (Winston et al. 1997, Barnes \& Sanderson 2000). On Macrocystis pyrifera holdfasts in Tasmanian waters, polychaetes are an abundant element of the rafting fauna hardly experiencing any losses in relative abundance over an observation period of about 6 months (Edgar 1987). Some of the most typical groups on floating items are spirorbid polychaetes, which often colonise attached macroalgae, and only secondarily, after detachment of algae, become rafters (Gee 1963). Spirorbid polychaetes colonise the blades (Knight-Jones et al. 1975) and holdfasts (Knight-Jones \& KnightJones 1984) of macroalgae, the surfaces of plastic items or even floating Nautilus shells (Hamada 1964, Chirat 2000). Distributional evidence suggests that spirorbid polychaetes can be transported over large distances on floating algae (Knight-Jones et al. 1975). The exceptionally wide distribution of the species Romanchella perrieri, Protolaeospira tricostalis and Paralaeospira levinseni is considered by Knight-Jones \& Knight-Jones (1984) a result of transport on floating Macrocystis pyrifera. Aliani \& Molcard (2003) found the serpulid polychaete Spirobranchus polytrema in $50 \%$ of their samples of floating items from the Ligurian Sea. This species was found exclusively on floating debris but not on macroalgae.

Yet not only sessile but also motile polychaete species are found on floating objects. In the Mediterranean Aliani \& Molcard (2003) collected Nereis falsa from rafting plants as well as from floating debris where both adults and juveniles occurred in about $50 \%$ of the samples. Arnaud et al. (1976) found two individuals of Perinereis capensis on floating kelp in the southeast Atlantic. The semi-sessile species Platynereis dumerilii is one of the dominant organisms on floating Sargassum 


\section{RAFTING OF BENTHIC MARINE ORGANISMS}

in the Sargasso Sea (Butler et al. 1983). The carnivorous species from the family Amphinomidae are frequently associated with stalked barnacles from floating items.

Oligochaetes occurred in considerable numbers on clumps of floating macroalgae in the waters around Iceland (Ingólfsson 1998) (Table 6). However, the number of individuals decreased with increasing floating duration, indicating that the neustonic environment provides unfavourable conditions for oligochaetes. Interestingly, Thornton (1997) mentioned that the first oligochaete found on Krakatau was a species living in decaying wood. Wittmann (1934) agrees with Benham (1902) that terrestrial and limnic oligochaetes are unlikely to be distributed between the southern hemisphere continents by rafting because the animals are not able to withstand immersion in sea water.

\section{Tardigrada}

One tardigrade species has been commonly found on pelagic Sargassum in the Sargasso Sea (Thulin 1942) (Table 6). Styraconix sarsi appears to be well adapted to the rafting environment but no other tardigrades have been reported from floating items.

\section{Arthropoda (Chelicerata)}

Spiders, scorpions and mites were collected from floating items in coastal waters of Puerto Rico (Heatwole \& Levins 1972) (Table 7). Niedbala (1998) inferred rafting as the most likely mechanism of dispersal of terrestrial moss mites among Pacific islands. Driftwood is considered the most suitable rafting substratum for pseudoscorpions from the maritime region of Baja California (Lee 1979). Ingólfsson (1998) reported Acari to be abundant on floating seaweeds.

Pycnogonids are common on floating Sargassum in the Sargasso Sea (Fage 1949, Fine 1970, Butler et al. 1983). Arnaud et al. (1976) found pycnogonids of the species Tanystylum brevipes on floating Ecklonia maxima near St. Helena. The finding of all developmental stages (adult males, adult females and juveniles) indicated that this species reproduced successfully on the raft. Small numbers of pycnogonids were also found by Bushing (1994) on floating macroalgae.

\section{Arthropoda (Insecta)}

Heatwole \& Levins (1972) reported a variety of different insect species on floating items collected in coastal waters of Puerto Rico (Table 7). They also remarked that many insect species, which they collected on floating items were wood-borers, among them larvae of wood-boring beetles and termites. Gathorne-Hardy \& Jones (2000) used distributional evidence to infer that most termites that colonised the Krakatau Islands had reached these via rafting. Wheeler (1916) reported on an assemblage of ants of the species Pheidole peregrina consisting of a single soldier, five workers and three young, winged females. The animals were carried on a tree trunk probably from the Brazilian mainland to the island of San Sebastian where it was found stranded. The importance of wood for rafting dispersal of insects is further underlined by observations from Abe (1984) who found ants, crickets, cockroaches and longhorn beetles on driftwood from Krakatau.

Davenport \& Rees (1993) suggested that insects land on seaweed patches, but then become trapped in the surface film of the water. They found insects in open water and also in floating weed patches but densities in neuston net samples from the Irish Sea were greater in samples that contained seaweeds. However, most of the insects they found were dead. Dead insects entangled with flotsam at the sea surface might serve as a valuable source of food for other rafting animals (Cheng \& Birch 1977, Locke \& Corey 1986).

Many different insect species (mainly Coleoptera and Diptera) contribute to the decomposition of macroalgae accumulating on beaches after the latter have become washed ashore (Griffiths \& Stenton-Dozey 1981, Inglis 1989). Some of these plants become resuspended in the water during 


\section{MARTIN THIEL \& LARS GUTOW}

Table 7 Arthropoda Chelicerata and Insecta reported or inferred as rafting

\begin{tabular}{|c|c|c|c|c|}
\hline Species & Region & $\begin{array}{l}\text { Rafting } \\
\text { inference }\end{array}$ & $\begin{array}{l}\text { Floating } \\
\text { substratum }\end{array}$ & Reference \\
\hline \multicolumn{5}{|l|}{ Acari } \\
\hline Atropacarus andrei & Pacific Islands & $\mathrm{D}$ & $\mathrm{W}$ & Niedbala 1998 \\
\hline Atropacarus glaucus & Pacific Islands & $\mathrm{D}$ & $\mathrm{W}$ & Niedbala 1998 \\
\hline Atropacarus rangiroaensis & Pacific Islands & $\mathrm{D}$ & $\mathrm{W}$ & Niedbala 1998 \\
\hline Atropacarus singularis & Pacific Islands & $\mathrm{D}$ & $\mathrm{W}$ & Niedbala 1998 \\
\hline Atropacarus stilifer & Pacific Islands & $\mathrm{D}$ & $\mathrm{W}$ & Niedbala 1998 \\
\hline Austrotritia lebronneci & Pacific Islands & $\mathrm{D}$ & $\mathrm{W}$ & Niedbala 1998 \\
\hline Camisia anomia & Spitzbergen & $\mathrm{C}$ & W & Coulson et al. 2002 \\
\hline Indotritia krakatauensis & Pacific Islands & $\mathrm{D}$ & $\mathrm{W}$ & Niedbala 1998 \\
\hline Plonaphacarus kugohi & Pacific Islands & $\mathrm{D}$ & $\mathrm{W}$ & Niedbala 1998 \\
\hline \multicolumn{5}{|l|}{ Araneae } \\
\hline Desis marina & Southern oceans & $\mathrm{C}$ & $\mathrm{W}$ & $\begin{array}{l}\text { Lawrence } 1971 \text { cited in Pugh } \\
2004\end{array}$ \\
\hline \multicolumn{5}{|l|}{ Pseudoscorpionida } \\
\hline Garypus californicus & Sea of Cortéz & $\mathrm{B}$ & $\mathrm{M}, \mathrm{W}$ & Lee 1979 \\
\hline Goryphus maldivensis & Krakatau Islands & $\mathrm{B}$ & $\mathrm{W}$ & Thornton 1997 \\
\hline \multicolumn{5}{|l|}{ Pycnogonida } \\
\hline Achelia assimilis & Central Chile & $\mathrm{D}$ & M & González \& Edding 1990b \\
\hline Anoplodactylus petiolatus & $\begin{array}{l}\text { Sargasso Sea, Gulf } \\
\text { Stream }\end{array}$ & $\mathrm{R}$ & M & $\begin{array}{l}\text { Fage 1949, Fine 1970, Butler et } \\
\text { al. 1983, Stoner \& Greening } \\
\text { 1984, Coston-Clements et al. } \\
\text { 1991 }\end{array}$ \\
\hline Endeis spinosa & $\begin{array}{l}\text { Northwest Atlantic, } \\
\text { Sargasso Sea }\end{array}$ & $\mathrm{R}$ & M & $\begin{array}{l}\text { Adams 1960, Fine 1970, Butler et } \\
\text { al. 1983, Coston-Clements et al. } \\
1991\end{array}$ \\
\hline Phoxichilus spinosus & $\begin{array}{l}\text { Sargasso Sea, } \\
\text { North Atlantic }\end{array}$ & $\mathrm{R}$ & M & Timmermann 1932*, Fage 1949 \\
\hline Pycnogonum stearnsi & California & $\mathrm{R}$ & M & Bushing 1994 \\
\hline Tanystylum brevipes & St. Helena & $\mathrm{R}$ & M & Arnaud et al. 1976 \\
\hline Tanystylum orbiculare & $\begin{array}{l}\text { Northwest Atlantic, } \\
\text { Sargasso Sea }\end{array}$ & $\mathrm{R}$ & M & $\begin{array}{l}\text { Adams 1960, Fine 1970, Butler et } \\
\text { al. 1983, Coston-Clements et al. } \\
1991\end{array}$ \\
\hline
\end{tabular}

Insecta, Collembola

Anurida maritima

Folsomia quadrioculata

Iceland

Spitzbergen

Hypogastrura tullbergi

Onychiurus arcticus

Onychiurus groenlandicus

Spitzbergen

Spitzbergen

Spitzbergen

Tetracanthella arctica

Spitzbergen

$\begin{array}{ll}\text { R } & \text { M } \\ \text { C } & \text { W } \\ \text { C } & \text { W } \\ \text { C } & \text { W } \\ \text { C } & \text { W } \\ \text { C } & \text { W }\end{array}$

Ingólfsson 1995

Coulson et al. 2002

Coulson et al. 2002

Coulson et al. 2002

Coulson et al. 2002

Coulson et al. 2002

Insecta, Diptera

Atrichopogon sp.

Cricotopus varipes

Puerto Rico

Iceland

Dasyhelea sp.

Puerto Rico

Dohrniphora sp.

Forcipomya raleighi

Puerto Rico

Puerto Rico

$\begin{array}{ll}\mathrm{R} & \mathrm{O} \\ \mathrm{R} & \mathrm{M} \\ \mathrm{R} & \mathrm{O} \\ \mathrm{R} & \mathrm{O} \\ \mathrm{R} & \mathrm{O}\end{array}$

Heatwole \& Levins 1972

Ingólfsson \& Kristjánsson 2002*

Heatwole \& Levins 1972

Heatwole \& Levins 1972

Heatwole \& Levins 1972 


\section{RAFTING OF BENTHIC MARINE ORGANISMS}

Table 7 (continued) Arthropoda Chelicerata and Insecta reported or inferred as rafting

\begin{tabular}{|c|c|c|c|c|}
\hline Species & Region & $\begin{array}{l}\text { Rafting } \\
\text { inference }\end{array}$ & $\begin{array}{l}\text { Floating } \\
\text { substratum }\end{array}$ & Reference \\
\hline Halocladius variabilis & Iceland & $\mathrm{R}$ & M & $\begin{array}{l}\text { Ingólfsson 1995, 1998, 2000, } \\
\text { Ingólfsson \& Kristjánsson } 2002\end{array}$ \\
\hline Puliciphora sp. & Puerto Rico & $\mathrm{R}$ & $\mathrm{O}$ & Heatwole \& Levins 1972 \\
\hline \multicolumn{5}{|l|}{ Insecta, Hymenoptera } \\
\hline Brachymyrmex obscurior & San Juan & $\mathrm{R}$ & $\mathrm{O}$ & Heatwole \& Levins 1972 \\
\hline Crematogaster steinheili & Puerto Rico & $\mathrm{R}$ & $\mathrm{O}$ & Heatwole \& Levins 1972 \\
\hline Glyptotermes caudomanitus & Krakatau Islands & B & $\mathrm{W}$ & Abe 1984 \\
\hline Nasutitermes matangensis & Krakatau Islands & B & W & Abe 1984 \\
\hline Paratrechina longicornis & San Juan & $\mathrm{R}$ & $\mathrm{O}$ & Heatwole \& Levins 1972 \\
\hline Pheidole peregrina & Sao Sebastian, Brazil & B & W & Wheeler 1916 \\
\hline Prorhinotermes sp. & Krakatau Islands & B & W & Abe 1984 \\
\hline \multicolumn{5}{|l|}{ Insecta, Coleoptera } \\
\hline Cercyon littoralis & Iceland & $\mathrm{R}$ & M & Ingólfssson 1995 \\
\hline Chalepides barbatus & Puerto Rico & $\mathrm{R}$ & $\mathrm{O}$ & Heatwole \& Levins 1972 \\
\hline Coleopa frigida & Iceland & $\mathrm{R}$ & M & Ingólfsson 1995 \\
\hline Macrancylus linearis & Puerto Rico & $\mathrm{R}$ & $\mathrm{O}$ & Heatwole \& Levins 1972 \\
\hline Omalium sp. & Iceland & $\mathrm{R}$ & M & Ingólfsson 1995 \\
\hline Sphenophorus sp. & Puerto Rico & $\mathrm{R}$ & $\mathrm{O}$ & Heatwole \& Levins 1972 \\
\hline
\end{tabular}

Notes: $\mathrm{B}=$ stranded floating item on beach; $\mathrm{C}=$ circumstantial; $\mathrm{D}=$ distributional inference; $\mathrm{M}=$ macroalgae; $\mathrm{O}=$ other; $\mathrm{R}=$ rafting; $\mathrm{W}=$ wood.

Please, note that insects from the genus Halobates are not considered in this list (see Andersen \& Cheng 2004 for most recent review on this group).

* References refer to this species under a different name.

extremely high tides probably carrying some insects with them to the open sea (Ingólfsson 1995). Yerbury (1919) considered the possibility that empids of the species Chersodromia hirta, which are commonly found under wet seaweed on British sandy shores, "make rafts of the seaweed and sail away to the sea" at high water. Dipteran insects were collected in low numbers from floating algal patches near the Washington State coast (Shaffer et al. 1995). Larvae of the dipteran Coelopa frigida (Ingólfsson 1995) and Halocladius variabilis (Ingólfsson 1998) were common or even massively abundant on algal rafts in Icelandic waters. However, the survival of eggs and larvae of beach-dwelling insects remains questionable when exposed to marine conditions. Coulson et al. (2002) found relatively high survival rates for several coastal species of springtails and suggested that these species are well-adapted for cross-oceanic dispersal. Resistance to contact with saltwater may be important for survival during rafting. In surface plankton tows Cheng \& Birch (1977) collected considerable numbers of insects in the Eastern Pacific and in the English Channel. They suggested that some of the animals might have rafted to the sea from freshwater sources. However, from the scarcity of floating objects during the sampling periods they concluded that the majority was probably airborne. Locke \& Corey (1986) adopted this argument in order to explain the origin of a diverse insect fauna collected in the Bay of Fundy (Canada) using a neuston net. But even though the amount of flotsam of terrestrial origin might have been quite low in the surface water, floating macroalgae were abundant during that cruise (Locke \& Corey 1989), potentially serving 


\section{MARTIN THIEL \& LARS GUTOW}

as suitable rafts for insects. Since large parts of the Bay of Fundy are surrounded by land masses, aerial introduction of insects is possible but rafting appears a reasonable explanation for the occurrence of numerous wingless insects or developmental stages (generally pupae) as well as spiders and mites in the central bay.

Species from the genus Halobates have assumed a holopelagic lifestyle. They skate autonomously over the water surface, but are often associated with floating items and drift lines where they find food and attachment sites for their eggs (see Andersen \& Cheng 2004 for further details).

\section{Arthropoda (Crustacea, Cladocera)}

In a compilation of animals commonly associated with Sargassum weed, Evadne spinifera is listed as the only cladoceran species (Adams 1960) (Table 8). However, it is doubtful whether this species can be considered as a rafter. Ingólfsson (1998) reported pelagic stages (larvae and adults) of several crustacean taxa such as cladocerans, calanoid copepods, cirripede larvae and decapod larvae. These taxa were not more common in samples of floating algae than in algal-free control hauls (see also Shaffer et al. 1995). Furthermore, unlike typical rafters no correlation was detected between the number of individuals and clump size. Hence, the author concluded that these taxa are not a priori associated with floating algae but were probably accidentally caught with seaweed.

\section{Arthropoda (Crustacea, Ostracoda)}

A few ostracod species have also been inferred to be dispersed via rafting (Peck 1994) (Table 8). The species Philomedes globosus has been found on floating macroalgae near Iceland (Ingólfsson 1995).

\section{Arthropoda (Crustacea, Copepoda)}

The lifestyle of most calanoid and cyclopoid copepods is planktonic. However, if copepods are found in clumps of floating seaweeds while they are missing from the surrounding plankton, as it was found for Microcalanus sp. (Shaffer et al. 1995), the animals might have used the substratum as a raft. Similar to calanoids, cyclopoid copepods, too, are incidentally found in floating seaweeds (e.g., Yeatman 1962, Hicks 1988).

In contrast to the two former groups, harpacticoid copepods are common rafting organisms, in particular on floating macroalgae (Yeatman 1962, Shaffer et al. 1995, Ingólfsson \& Ólafsson 1997, Ólafsson et al. 2001) (Table 8). From floating algae Ascophyllum nodosum and Fucus vesiculosus caught in coastal waters of Iceland, Ólafsson et al. (2001) reported 38 species of harpacticoid copepods, most of which usually inhabit benthic macroalgae. Some of the commonly encountered species are also found associated with sandy or muddy bottoms but none of these species is known to exclusively dwell in muddy or sandy habitats. As in other meiofaunal species such as nematodes, rafting of harpacticoid copepods might be the result of incidental encounter of the animals with floating structures during active or passive suspension in the water column. While active movements of copepods on algal rafts are mostly restricted to short-distance swimming from branch to branch within the algal patch, Yeatman (1962) considered it as quite probable that the animals could also swim short distances from clump to clump. This might have important effects on the dynamics, (genetic) structure and persistence of local populations on algal patches that are temporally connected by this mechanism.

Harpacticoid copepods rapidly colonised macroalgae tethered in nearshore habitats (Ólafsson et al. 2001). Within 8 or 9 days, the density of harpacticoids increased significantly. After the same period the harpacticoid community was more diverse on floating algal clumps than on attached algae of the same species. The floating macroalgae are used as shelter, as feeding and also as a 


\section{RAFTING OF BENTHIC MARINE ORGANISMS}

Table 8 Arthropoda Branchiopoda and Maxillopoda reported or inferred as rafting

\begin{tabular}{|c|c|c|c|c|}
\hline Species & Region & $\begin{array}{l}\text { Rafting } \\
\text { inference }\end{array}$ & $\begin{array}{l}\text { Floating } \\
\text { substratum }\end{array}$ & Reference \\
\hline \multicolumn{5}{|l|}{ Branchiopoda } \\
\hline Evadne spinifera & $\begin{array}{l}\text { Northwest Atlantic, } \\
\text { Sargasso Sea }\end{array}$ & $\mathrm{R}$ & M & $\begin{array}{l}\text { Adams 1960, Coston-Clements } \\
\text { et al. } 1991\end{array}$ \\
\hline Evadne sp. & U.S. West Coast & $\mathrm{R}$ & M & Shaffer et al. 1995 \\
\hline Podon sp. & U.S. West Coast & $\mathrm{R}$ & M & Shaffer et al. 1995 \\
\hline \multicolumn{5}{|l|}{ Ostracoda } \\
\hline Anchistrocheles darwini & Galapagos & $\mathrm{D}$ & n.i. & Peck 1994 \\
\hline Occultocythereis augusta & Galapagos & $\mathrm{D}$ & n.i. & Peck 1994 \\
\hline Paracypris crispa & Galapagos & $\mathrm{D}$ & n.i. & Peck 1994 \\
\hline Philomedes globosus & Iceland & $\mathrm{R}$ & $\mathrm{M}$ & Ingólfsson 1995 \\
\hline \multicolumn{5}{|l|}{ Copepoda, Cyclopoida } \\
\hline Copilia mediterranea & $\begin{array}{l}\text { Northwest Atlantic, } \\
\text { Sargasso Sea }\end{array}$ & $\mathrm{R}$ & M & $\begin{array}{l}\text { Adams } 1960 \text {, Coston-Clements } \\
\text { et al. } 1991\end{array}$ \\
\hline Corycaeus sp. & U.S. West Coast & $\mathrm{R}$ & M & Shaffer et al. 1995 \\
\hline Macrochiron sargassi & Sargasso Sea, Gulf Stream & $\mathrm{R}$ & M & $\begin{array}{l}\text { Butler et al. 1983, Coston- } \\
\text { Clements et al. } 1991\end{array}$ \\
\hline Oithona sp. & U.S. West Coast & $\mathrm{R}$ & M & Shaffer et al. 1995 \\
\hline Oncaea sp. & U.S. West Coast & $\mathrm{R}$ & M & Shaffer et al. 1995 \\
\hline $\begin{array}{l}\text { Pseudomacrochiron } \\
\text { parvum }\end{array}$ & Sargasso Sea & $\mathrm{R}$ & M & Butler et al. 1983 \\
\hline \multicolumn{5}{|l|}{ Copepoda, Calanoida } \\
\hline Acartia sp. & U.S. West Coast & $\mathrm{R}$ & M & Shaffer et al. 1995 \\
\hline Anomalocera patersoni & Irish Sea & $\mathrm{R}$ & M & Davenport \& Rees 1993 \\
\hline Calanus sp. & U.S. West Coast & $\mathrm{R}$ & M & Shaffer et al. 1995 \\
\hline Centropages typicus & Irish Sea & $\mathrm{R}$ & M & Davenport \& Rees 1993 \\
\hline Centropages sp. & U.S. West Coast & $\mathrm{R}$ & M & Shaffer et al. 1995 \\
\hline Microcalanus sp. & U.S. West Coast & $\mathrm{R}$ & M & Shaffer et al. 1995 \\
\hline Paracalanus sp. & U.S. West Coast & $\mathrm{R}$ & M & Shaffer et al. 1995 \\
\hline Pseudocalanus sp. & U.S. West Coast & $\mathrm{R}$ & M & Shaffer et al. 1995 \\
\hline \multicolumn{5}{|l|}{ Copepoda, Harpacticoida } \\
\hline Ameira longipes & Iceland & $\mathrm{R}$ & M & Ólafsson et al. 2001 \\
\hline Amonardia arctica & Iceland & $\mathrm{R}$ & M & Ólafsson et al. 2001 \\
\hline Amonardia phyllopus & Sargasso Sea, Gulf Stream & $\mathrm{R}$ & M & $\begin{array}{l}\text { Yeatman 1962, Butler et al. 1983, } \\
\text { Coston-Clements et al. } 1991\end{array}$ \\
\hline Amphiascoides debilis & Iceland & $\mathrm{R}$ & M & Ólafsson et al. 2001 \\
\hline Amphiascoides nanus & Iceland & $\mathrm{R}$ & M & Ólafsson et al. 2001 \\
\hline Amphiascus minutus & Iceland & $\mathrm{R}$ & M & Ólafsson et al. 2001 \\
\hline Brianola cf. pori & New Zealand & $\mathrm{R}$ & $\mathrm{O}$ & Hicks 1988 \\
\hline Dactylopodia tisboides & $\begin{array}{l}\text { Sargasso Sea, Gulf } \\
\text { Stream, New Zealand }\end{array}$ & $\mathrm{R}$ & M & $\begin{array}{l}\text { Yeatman 1962, Hicks 1977, Butler } \\
\text { et al. 1983, Coston-Clements } \\
\text { et al. } 1991\end{array}$ \\
\hline Dactylopodia sp. & U.S. West Coast & $\mathrm{R}$ & M & Shaffer et al. 1995 \\
\hline Dactylopusia vulgaris & Iceland & $\mathrm{R}$ & M & Ólafsson et al. 2001 \\
\hline Diarthrodes major & Iceland & $\mathrm{R}$ & M & Ólafsson et al. 2001 \\
\hline Diarthrodes nobilis & Iceland & $\mathrm{R}$ & M & Ólafsson et al. 2001 \\
\hline
\end{tabular}




\section{MARTIN THIEL \& LARS GUTOW}

Table 8 (continued) Arthropoda Branchiopoda and Maxillopoda reported or inferred as rafting

\begin{tabular}{|c|c|c|c|c|}
\hline Species & Region & $\begin{array}{l}\text { Rafting } \\
\text { inference }\end{array}$ & $\begin{array}{l}\text { Floating } \\
\text { substratum }\end{array}$ & Reference \\
\hline Diosaccus aff. varicolor & Iceland & $\mathrm{R}$ & M & Ólafsson et al. 2001 \\
\hline Diosaccus sp. & Iceland & $\mathrm{R}$ & M & Ólafsson et al. 2001 \\
\hline Ectinosoma melaniceps & New Zealand & $\mathrm{R}$ & $\mathrm{O}$ & Hicks 1988 \\
\hline Ectinosoma sp. & Iceland & $\mathrm{R}$ & M & Ólafsson et al. 2001 \\
\hline Enhydrosoma variabile & New Zealand & $\mathrm{R}$ & $\mathrm{O}$ & Hicks 1988 \\
\hline Euterpina acutifrons & New Zealand & $\mathrm{R}$ & $\mathrm{O}$ & Hicks 1988 \\
\hline Halectinosoma hydrofuge & New Zealand & $\mathrm{R}$ & $\mathrm{O}$ & Hicks 1988 \\
\hline Halectinosoma otakoua & New Zealand & $\mathrm{R}$ & $\mathrm{O}$ & Hicks 1988 \\
\hline Harpacticus chelifer & Iceland & $\mathrm{R}$ & M & Ólafsson et al. 2001 \\
\hline Harpacticus flexus & Iceland & $\mathrm{R}$ & M & Ólafsson et al. 2001 \\
\hline Harpacticus gurneyi & Sargasso Sea, Gulf Stream & $\mathrm{R}$ & M & $\begin{array}{l}\text { Yeatman 1962, Butler et al. 1983, } \\
\text { Coston-Clements et al. } 1991\end{array}$ \\
\hline Harpacticus obscurus & Iceland & $\mathrm{R}$ & M & Ólafsson et al. 2001 \\
\hline $\begin{array}{l}\text { Harpacticus } \\
\text { septentrionalis }\end{array}$ & Iceland & $\mathrm{R}$ & M & Ólafsson et al. 2001 \\
\hline Harpacticus uniremis & Iceland & $\mathrm{R}$ & M & Ólafsson et al. 2001 \\
\hline Harpacticus sp. & New Zealand & $\mathrm{R}$ & $\mathrm{O}$ & Hicks 1988 \\
\hline Harpacticus sp. & U.S. West Coast & $\mathrm{R}$ & M & Shaffer et al. 1995 \\
\hline $\begin{array}{l}\text { Heterolaophonte } \\
\text { littoralis }\end{array}$ & Iceland & $\mathrm{R}$ & M & Ólafsson et al. 2001 \\
\hline $\begin{array}{l}\text { Heterolaophonte } \\
\text { longisetigera }\end{array}$ & Iceland & $\mathrm{R}$ & M & Ólafsson et al. 2001 \\
\hline Heterolaophonte strömi & Iceland & $\mathrm{R}$ & M & Ólafsson et al. 2001 \\
\hline Itunella sp. & Iceland & $\mathrm{R}$ & M & Ólafsson et al. 2001 \\
\hline Laophonte setosa & Iceland & $\mathrm{R}$ & M & Ólafsson et al. 2001 \\
\hline Laophonte sp. & U.S. West Coast & $\mathrm{R}$ & M & Shaffer et al. 1995 \\
\hline Lourinia armata & New Zealand & $\mathrm{R}$ & $\mathrm{O}$ & Hicks 1988 \\
\hline Mesochra pygmaea & Iceland & $\mathrm{R}$ & M & Ólafsson et al. 2001 \\
\hline Mesochra sp. & U.S. West Coast & $\mathrm{R}$ & M & Shaffer et al. 1995 \\
\hline Microsetella norvegica & Iceland & $\mathrm{R}$ & M & Ólafsson et al. 2001 \\
\hline Microsetella rosea & New Zealand & $\mathrm{R}$ & $\mathrm{O}$ & Hicks 1988 \\
\hline Miscegenus heretaunga & New Zealand & $\mathrm{R}$ & $\mathrm{O}$ & Hicks 1988 \\
\hline Nitokra typica & Iceland & $\mathrm{R}$ & M & Ólafsson et al. 2001 \\
\hline Paradactylopodia oculata & Sargasso Sea, Gulf Stream & $\mathrm{R}$ & M & $\begin{array}{l}\text { Yeatman 1962, Butler et al. 1983, } \\
\text { Coston-Clements et al. } 1991\end{array}$ \\
\hline Paradactylopodia sp. & U.S. West Coast & $\mathrm{R}$ & M & Shaffer et al. 1995 \\
\hline $\begin{array}{l}\text { Paralaophonte } \\
\text { aenigmaticum }\end{array}$ & New Zealand & $\mathrm{R}$ & $\mathrm{O}$ & Hicks 1988 \\
\hline Paralaophonte congenera & $\begin{array}{l}\text { Sargasso Sea, Gulf } \\
\text { Stream, New Zealand }\end{array}$ & $\mathrm{R}$ & M & $\begin{array}{l}\text { Yeatman 1962, Hicks 1977, Butler } \\
\text { et al. 1983, Coston-Clements } \\
\text { et al. } 1991\end{array}$ \\
\hline Paralaophonte perplexa & Iceland & $\mathrm{R}$ & M & Ólafsson et al. 2001 \\
\hline Paralaophonte sp. & New Zealand & $\mathrm{R}$ & $\mathrm{O}$ & Hicks 1988 \\
\hline Paraleptastacus sp. & New Zealand & $\mathrm{R}$ & $\mathrm{O}$ & Hicks 1988 \\
\hline Paramphiascella hispida & Iceland & $\mathrm{R}$ & M & Ólafsson et al. 2001 \\
\hline $\begin{array}{l}\text { Paramphiascopsis } \\
\text { giesbrechti }\end{array}$ & Iceland & $\mathrm{R}$ & M & Ólafsson et al. 2001 \\
\hline $\begin{array}{l}\text { Parastenhelia } \\
\text { megarostrum }\end{array}$ & New Zealand & $\mathrm{R}$ & $\mathrm{O}$ & Hicks 1988 \\
\hline
\end{tabular}




\section{RAFTING OF BENTHIC MARINE ORGANISMS}

Table 8 (continued) Arthropoda Branchiopoda and Maxillopoda reported or inferred as rafting

\begin{tabular}{|c|c|c|c|c|}
\hline Species & Region & $\begin{array}{l}\text { Rafting } \\
\text { inference }\end{array}$ & $\begin{array}{l}\text { Floating } \\
\text { substratum }\end{array}$ & Reference \\
\hline Parastenhelia spinosa & Iceland & $\mathrm{R}$ & M & Ólafsson et al. 2001 \\
\hline Parathalestris clausi & Iceland & $\mathrm{R}$ & M & Ólafsson et al. 2001 \\
\hline Parathalestris croni & Island & $\mathrm{R}$ & M & $\begin{array}{l}\text { Ingólfsson 1995, 1998, 2000, } \\
\text { Ingólfsson \& Ólafsson 1997, } \\
\text { Ólafsson et al. } 2001\end{array}$ \\
\hline Parathalestris intermedia & Iceland & $\mathrm{R}$ & M & Ólafsson et al. 2001 \\
\hline Parathalestris sp. & U.S. West Coast & $\mathrm{R}$ & M & Shaffer et al. 1995 \\
\hline $\begin{array}{l}\text { Paronychocamptus } \\
\text { curticaudatus }\end{array}$ & Iceland & $\mathrm{R}$ & M & Ólafsson et al. 2001 \\
\hline Porcellidium sp. & New Zealand & $\mathrm{R}$ & $\mathrm{O}$ & Hicks 1988 \\
\hline Porcellidium sp. & U.S. West Coast & $\mathrm{R}$ & M & Shaffer et al. 1995 \\
\hline $\begin{array}{l}\text { Pseudonychocamptus } \\
\text { koreni }\end{array}$ & Iceland & $\mathrm{R}$ & M & Ólafsson et al. 2001 \\
\hline Psyllocamptus minutus & New Zealand & $\mathrm{R}$ & $\mathrm{O}$ & Hicks 1988 \\
\hline $\begin{array}{l}\text { Rhynchothalestris } \\
\text { helgolandica }\end{array}$ & Iceland & $\mathrm{R}$ & M & Ólafsson et al. 2001 \\
\hline Robertsonia propinqua & New Zealand & $\mathrm{R}$ & $\mathrm{O}$ & Hicks 1988 \\
\hline Robertsonia sp. & Iceland & $\mathrm{R}$ & M & Ólafsson et al. 2001 \\
\hline Scutellidium longicauda & $\begin{array}{l}\text { Sargasso Sea, Gulf } \\
\text { Stream, New Zealand }\end{array}$ & $\mathrm{R}$ & M & $\begin{array}{l}\text { Yeatman 1962, Hicks 1977, Butler } \\
\text { et al. 1983, Coston-Clements } \\
\text { et al. } 1991\end{array}$ \\
\hline Thalestris longimana & Iceland & $\mathrm{R}$ & M & Ólafsson et al. 2001 \\
\hline Tisbe furcata & New Zealand & $\mathrm{R}$ & $\mathrm{O}$ & Hicks 1988 \\
\hline Tisbe holothuriae & New Zealand & $\mathrm{R}$ & $\mathrm{O}$ & Hicks 1988 \\
\hline Tisbe sp. & U.S. West Coast & $\mathrm{R}$ & M & Shaffer et al. 1995 \\
\hline Tisbe spp. & Iceland & $\mathrm{R}$ & M & Ólafsson et al. 2001 \\
\hline Zaus aurelii & Iceland & $\mathrm{R}$ & M & Ólafsson et al. 2001 \\
\hline Zaus sp. & U.S. West Coast & $\mathrm{R}$ & M & Shaffer et al. 1995 \\
\hline \multicolumn{5}{|l|}{ Cirripedia } \\
\hline Alepas pacifica & Sagami Bay, Japan & $\mathrm{R}$ & M & Hirosaki 1964 \\
\hline Balanus amphitrite & Panama & $\mathrm{R}$ & $\mathrm{W}$ & Donlan \& Nelson 2003 \\
\hline $\begin{array}{l}\text { Balanus amphitrite } \\
\text { amphitrite }\end{array}$ & Florida & $\mathrm{B}$ & $\mathrm{P}$ & Winston et al. 1997 \\
\hline Balanus eburneus & Florida & B & $\mathrm{P}$ & Winston et al. 1997 \\
\hline Balanus trigonus & Sagami Bay, Japan & $\mathrm{R}$ & M & Hirosaki 1964 \\
\hline Conchoderma auritum & Azores & $\mathrm{R}$ & $\mathrm{M}, \mathrm{O}, \mathrm{P}, \mathrm{W}$ & $\begin{array}{l}\text { Nilsson-Cantell 1930, Il'in 1992, } \\
\text { Morton \& Britton } 2000\end{array}$ \\
\hline Conchoderma virgatum & $\begin{array}{l}\text { Northwest Atlantic, } \\
\text { Sargasso Sea, Sagamy } \\
\text { Bay, Japan, off Costa } \\
\text { Rica }\end{array}$ & $\mathrm{R}$ & $\mathrm{M}, \mathrm{O}, \mathrm{W}$ & $\begin{array}{l}\text { Nilsson-Cantell 1930, Adams } \\
\text { 1960, Hirosaki 1964, Hunter \& } \\
\text { Mitchell 1967, Butler et al. 1983, } \\
\text { Coston-Clements et al. 1991, } \\
\text { Il'in 1992, Morton \& Britton } \\
2000\end{array}$ \\
\hline Coronula diadema & Azores & $\mathrm{R}$ & $\mathrm{M}, \mathrm{P}, \mathrm{W}$ & Morton \& Britton 2000 \\
\hline Dosima fascicularis & Ireland, Azores & $\mathrm{B}, \mathrm{R}$ & $\mathrm{M}, \mathrm{O}, \mathrm{P}, \mathrm{W}$ & $\begin{array}{l}\text { Minchin 1996, Morton \& Britton } \\
\text { 2000, Hobday 2000a }\end{array}$ \\
\hline Elminius modestus & North Sea & B & $\mathrm{O}$ & Cadée 2002 \\
\hline Euraphia eastropacensis & Panama & $\mathrm{R}$ & $\mathrm{W}$ & Donlan \& Nelson 2003 \\
\hline
\end{tabular}




\section{MARTIN THIEL \& LARS GUTOW}

Table 8 (continued) Arthropoda Branchiopoda and Maxillopoda reported or inferred as rafting

\begin{tabular}{|c|c|c|c|c|}
\hline Species & Region & $\begin{array}{l}\text { Rafting } \\
\text { inference }\end{array}$ & $\begin{array}{l}\text { Floating } \\
\text { substratum }\end{array}$ & Reference \\
\hline Lepas anatifera & $\begin{array}{l}\text { Northwest Atlantic, West } \\
\text { Wind Drift, St. Helena, } \\
\text { Sargasso Sea, Falkland } \\
\text { Current, Sagamy Bay, } \\
\text { Japan, British Channel, } \\
\text { Red Sea, Ireland, Azores, } \\
\text { off San Benedicto, North } \\
\text { Pacific, Argentina, } \\
\text { Florida, New Zealand }\end{array}$ & $\mathrm{B}, \mathrm{R}$ & $\mathrm{M}, \mathrm{O}, \mathrm{P}, \mathrm{V}, \mathrm{W}$ & $\begin{array}{l}\text { Hodgson 1896, Hentschel 1922, } \\
\text { Nilsson-Cantell 1930, Richards } \\
\text { 1958, Adams 1960, Hirosaki } \\
\text { 1964, Markkaveeva 1965, } \\
\text { Coombs \& Landis 1966, Fine } \\
\text { 1970, Arnaud 1973, Arnaud et al. } \\
\text { 1976, Sinitsyn \& Reznichenko } \\
\text { 1981, Butler et al. 1983, Coston- } \\
\text { Clements et al. } 1991\end{array}$ \\
\hline Lepas anserifera & $\begin{array}{l}\text { Northwest Atlantic, } \\
\text { Sargasso Sea, Great } \\
\text { Barrier Reef, Brazil } \\
\text { Current, Sagami Bay, } \\
\text { Japan, North Pacific }\end{array}$ & $\mathrm{R}$ & $\mathrm{M}, \mathrm{O}$ & $\begin{array}{l}\text { Hentschel 1922, Timmermann } \\
\text { 1932, Adams 1960, Hirosaki } \\
\text { 1964, Fine 1970, Sinitsyn \& } \\
\text { Reznichenko 1981, Butler et al. } \\
\text { 1983, Coston-Clements et al. } \\
\text { 1991, DeVantier 1992, Il'in 1992, } \\
\text { Sano et al. } 2003\end{array}$ \\
\hline Lepas australis & $\begin{array}{l}\text { Scotia Arc, Falkland } \\
\text { Current, Sargasso Sea }\end{array}$ & $\mathrm{R}$ & $\mathrm{M}, \mathrm{O}$ & $\begin{array}{l}\text { Hentschel 1922, Nilsson-Cantell } \\
\text { 1930, Coston-Clements et al. } \\
\text { 1991, Il'in 1992, Helmuth et al. } \\
\text { 1994 }\end{array}$ \\
\hline Lepas beringians & & $\mathrm{R}$ & $\mathrm{O}$ & Il'in 1992 \\
\hline Lepas fascicularis & $\begin{array}{l}\text { Sargasso Sea, Japan, North } \\
\text { Pacific, North Sea }\end{array}$ & $\mathrm{R}$ & $\mathrm{M}, \mathrm{O}$ & $\begin{array}{l}\text { Thörner \& Ankel 1966, Fine 1970, } \\
\text { Sinitsyn \& Reznichenko 1981, } \\
\text { Butler et al. 1983, Coston- } \\
\text { Clements et al. 1991, Sano et al. } \\
2003\end{array}$ \\
\hline Lepas hilli & Sargasso Sea, Azores & $\mathrm{R}$ & $\mathrm{M}, \mathrm{O}, \mathrm{P}, \mathrm{W}$ & $\begin{array}{l}\text { Butler et al. 1983, Coston- } \\
\text { Clements et al. 1991, Il'in 1992, } \\
\text { Morton \& Britton } 2000\end{array}$ \\
\hline Lepas pectinata & $\begin{array}{l}\text { Northwest Atlantic, } \\
\text { Mediterranean, Sargasso } \\
\text { Sea, Sagami Bay, Japan, } \\
\text { Rhodes to Azores, } \\
\text { Ireland, North Pacific, } \\
\text { Northwest Pacific, } \\
\text { Florida }\end{array}$ & $\mathrm{B}, \mathrm{R}$ & $\mathrm{M}, \mathrm{O}, \mathrm{P}, \mathrm{T}, \mathrm{W}$ & $\begin{array}{l}\text { Hentschel 1922, Timmermann } \\
\text { 1932, Adams 1960, Hirosaki } \\
\text { 1964, Fine 1970, Horn et al. } \\
\text { 1970, Butler 1975, Sinitsyn \& } \\
\text { Reznichenko 1981, Butler et al. } \\
\text { 1983, Coston-Clements et al. } \\
\text { 1991, Il'in 1992, Minchin 1996, } \\
\text { Winston et al. 1997, Morton \& } \\
\text { Britton 2000, Tsikhon-Lukanina } \\
\text { et al. 2001a, Aliani \& Molcard } \\
2003\end{array}$ \\
\hline Lepas testudinata & & $\mathrm{R}$ & $\mathrm{O}$ & Il'in 1992 \\
\hline Lepas spp. & California & $\mathrm{R}$ & M & Hobday 2000a \\
\hline $\begin{array}{c}\text { Megabalanus } \\
\text { californicus }\end{array}$ & California & $\mathrm{R}$ & M & Hobday 2000a \\
\hline Xenobalanus gobicipitis & Azores & $\mathrm{R}$ & $\mathrm{M}, \mathrm{P}, \mathrm{W}$ & Morton \& Britton 2000 \\
\hline
\end{tabular}

Notes: $\mathrm{B}=$ stranded floating item on beach; $\mathrm{D}=$ distributional inference; $\mathrm{M}=$ macroalgae; n.i. $=$ no information; $\mathrm{O}=$ other; $\mathrm{P}=$ plastics $; \mathrm{R}=$ rafting; $\mathrm{T}=$ tar lumps $\mathrm{V}=$ volcanic pumice; $\mathrm{W}=$ wood. 


\section{RAFTING OF BENTHIC MARINE ORGANISMS}

reproductive habitat (Ingólfsson \& Ólafsson 1997). Females of the large pelagic species Parathalestris croni deposit their offspring (non-swimming nauplii) in floating macroalgae that serve as a nest for this species (Ingólfsson \& Ólafsson 1997). This nest function of algal clumps could not be observed for Harpacticus chelifer, which was found by Ólafsson et al. (2001) to be by far the most abundant harpactocoid species on floating algae near Iceland, occurring in virtually all floating fronds collected during the summer months.

Rafting on macroalgae may also serve as an efficient dispersal mechanism between distant locations (Yeatman 1962, Ólafsson et al. 2001). Since harpacticoid copepods are able to cling very tightly to algae (Monk 1941 cited in Yeatman 1962) they seem to be well equipped for rafting on macroalgae. The same behaviour has been described for nauplii of Tisbe furcata by Johnson \& Olson (1948). This species produces benthic larvae that use their prehensile hooks and second antennae to cling tightly to detritus particles. This indicates that generations of harpacticoid copepod species might be able to cover large distances by rafting because even juvenile stages do not get lost during the journey. Harpacticoid copepods represented more than $90 \%$ of the total fauna collected from sediment patches floating in the surface water of the Pauatahanui Inlet (Wellington, New Zealand) while cyclopoids made up only a very small portion ( 0.1\%) (Hicks 1988). A total of 21 harpacticoid species were found with more than $95 \%$ belonging to the epibenthic species Parastenhelia megarostrum, which occurred in all samples. Even though all developmental stages were found on sediment rafts, the overwhelming majority consisted of juveniles (nauplii and copepodites) among which the larval stages dominated. However, on adjacent benthic (non-floating) substrata, proportions of larvae were much lower indicating that larger or older individuals are less likely to raft probably due to their burrowing habit in contrast to the nauplii, which crawl on the sediment surface and cling to sand particles. A survivorship of more than $75 \%$ after a period of $18 \mathrm{~h}$ following sampling and handling in the laboratory suggested that nauplii were not negatively affected by sediment rafting.

\section{Arthropoda (Crustacea, Cirripedia)}

Barnacles are some of the most typical rafting organisms (Table 8). Stalked barnacles are found on macroalgae (Helmuth et al. 1994, Adams 1960, Nilsson-Cantell 1930), wood (Hodgson 1896, Nilsson-Cantell 1930), floating corals (Kornicker \& Squires 1962, DeVantier 1992), empty Nautilus shells (Chirat 2000), volcanic pumice (Richards 1958, Jokiel 1984, 1989), tar balls (Horn et al. 1970, Wong et al. 1974, Minchin 1996), glass fishing floats (Dell 1964) and plastic items (Arnaud 1973, Aliani \& Molcard 2003). Three species of the genus Lepas (L. anserifera, L. pectinata and L. anatifera) and one species of the genus Conchoderma (C. virgatum) are listed as members of the Sargassum community (Hentschel 1922, Adams 1960, Butler et al. 1983). Lepadid barnacles can form dense assemblages on floating substrata (Figure 3). Nilsson-Cantell (1930) provided images of (balanomorph as well as lepadomorph) barnacles growing on the skin and on baleen plates of whales. These barnacles inevitably become rafters during postmortem floating of their hosts. Lepas anatifera was the most abundant rafter found on floating kelp near St. Helena (Arnaud et al. 1976). Near Bermuda, Butler (1975) counted 150 individuals of L. pectinata (2-8 $\mathrm{mm}$ in length) from only four tar balls. From the size and the growth rates of the barnacles, he estimated the tar balls to be at least 2 months old. Lepadids possess pelagic larvae that colonise any floating item during the early stages of succession (Tsikhon-Lukanina et al. 2001b). Concentrations of lepadid larvae in general appear to be relatively low but exposed items nevertheless are colonised rapidly and in high densities (Il'in et al. 1977). Most Lepadidae are obligate rafters and their populations are entirely pelagic. For L. fascicularis rafting is an obligate but only transient developmental stage (Thörner \& Ankel 1966). Floating objects (mainly macroalgae but also terrestrial

objects such as wood and seeds of vascular plants) serve only for a short time period after attachment 


\section{MARTIN THIEL \& LARS GUTOW}

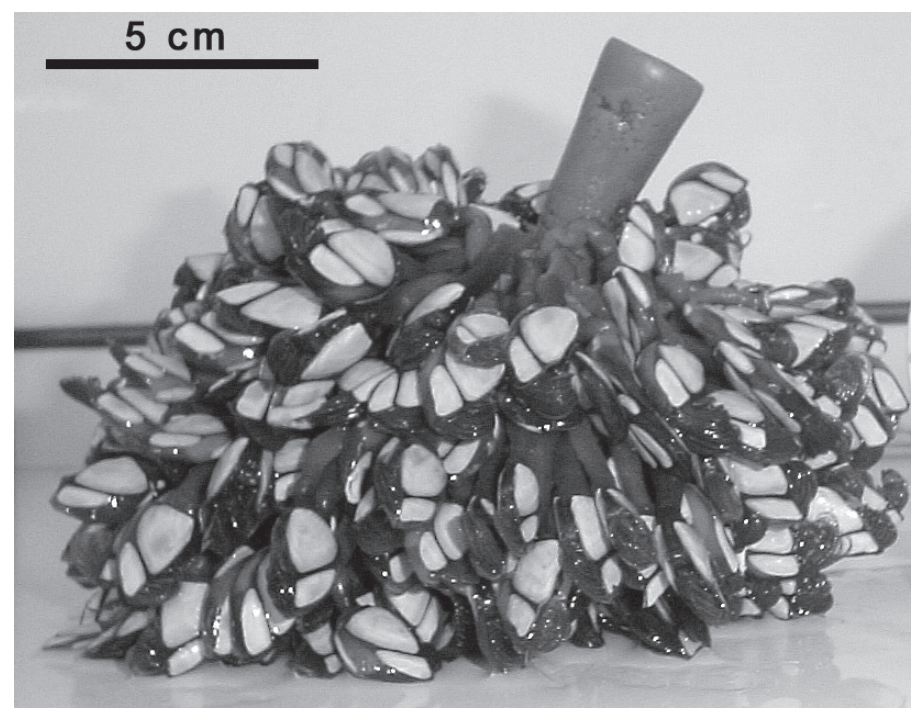

Figure 3 Dense colony of Lepas australis on holdfast of floating Durvillaea antarctica collected in coastal waters of the southeast Pacific (S Chile).

of a pelagic cypris larva as a raft. Soon after attachment the young barnacle secretes an air-filled, foamy mucus float that enables the organism to float autonomously. The former raft remains incorporated in the mucus float.

Benthic Cirripedia are also occasionally found on floating items, in particular on plastics and other abiotic items (Hirosaki 1964, Winston et al. 1997, Hobday 2000a).

\section{Arthropoda (Crustacea, Decapoda)}

Crabs and shrimp occur on many different substrata (Table 9). Shrimp can be common on macroalgae (M. Thiel, personal observation) and some decapod crabs are also abundant on kelp holdfasts (Edgar 1987, Vásquez 1993, Hobday 2000a) and other floating items (Schubart et al. 2001). The first benthic stages of many decapods may recruit to floating items, such as, for example, mangrove leaves (e.g., Wehrtmann \& Dittel 1990), macroalgae (M. Thiel, personal observation), and tar balls (Wong et al. 1974). Wehrtmann \& Dittel (1990) reported that larvae and juveniles of decapod crustaceans (shrimp as well as crabs) raft on mangrove leaves in Costa Rica. Within the Natantia, species of the genus Penaeus (unrevised) and Macrobrachium were predominant. Among the brachyuran crabs, megalopae of $U c a$ sp. were by far the most abundant taxon. Leaves were collected during ebb and flood tides. Significantly more animals were found on the leaves during flood (see also Schwamborn \& Bonecker 1996). Consequently, rafting is a mechanism to save energy while immigrating into the lagoon. Shanks (1985) described the behaviour of megalopae of the crab Pachygrapsus crassipes that inhabit the neuston where they cling to positively buoyant flotsam. If the assemblage of megalopae and flotsam is captured in a surface slick resulting from shoreward moving internal waves it will be transported towards shallow coastal waters where megalopae metamorphose to the first benthic stage. Based on these examples and the riding of megalopae of Cancer magister on hydroid medusae (Wickham 1979), Anger (2001) concluded that 'hitchhiking' might be a common means of larval transport in decapods. Additionally, leaves and other floating items serve as protection against predation of decapod larvae by fish. Larvae and megalopae of swimming crabs (Portunidae) are very abundant on floating algae in the northeast Atlantic region 


\section{RAFTING OF BENTHIC MARINE ORGANISMS}

Table 9 Arthropoda Malacostraca reported or inferred as rafting

\begin{tabular}{|c|c|c|c|c|}
\hline Species & Region & $\begin{array}{l}\text { Rafting } \\
\text { inference }\end{array}$ & $\begin{array}{l}\text { Floating } \\
\text { substratum }\end{array}$ & Reference \\
\hline \multicolumn{5}{|l|}{ Leptostraca } \\
\hline Nebalia pugettensis & NW coast of USA & $\mathrm{R}$ & M & Highsmith 1985 \\
\hline Nebalia sp. & Tasmania & $\mathrm{R}$ & M & Edgar 1987 \\
\hline \multicolumn{5}{|l|}{ Euphausiacea } \\
\hline Euphausia pacifica & California, Japan & $\mathrm{R}$ & M & Hobday 2000a, Sano et al. 2003 \\
\hline Nyctiphanes couchi & Irish Sea & $\mathrm{R}$ & M & Davenport \& Rees 1993 \\
\hline \multicolumn{5}{|l|}{ Decapoda, Anomura } \\
\hline Calcinus explorator & Galapagos & $\mathrm{D}$ & n.i. & Peck 1994 \\
\hline Coenobita compressus & Galapagos & $\mathrm{D}$ & n.i. & Peck 1994* \\
\hline Pachycheles pubescens & California & $\mathrm{R}$ & M & Hobday 2000a \\
\hline Pagurus bernhardus & Irish Sea & $\mathrm{R}$ & M & Davenport \& Rees 1993 \\
\hline Pagurus prideaux & Irish Sea & $\mathrm{R}$ & M & Davenport \& Rees 1993 \\
\hline \multicolumn{5}{|l|}{ Decapoda, Brachyura } \\
\hline Callinectes sp. & Costa Rica & $\mathrm{R}$ & $\mathrm{O}$ & Wehrtmann \& Dittel 1990 \\
\hline Callinectes spp. & Panama & $\mathrm{R}$ & $\mathrm{W}$ & Donlan \& Nelson 2003 \\
\hline Cancer productus & NW coast of USA & $\mathrm{R}$ & M & Highsmith 1985 \\
\hline Cancer sp. & California & $\mathrm{R}$ & M & Hobday 2000a \\
\hline Charybdis acuta & Sagami Bay, Japan & $\mathrm{R}$ & M & Hirosaki 1964 \\
\hline Cronius ruber & Sargasso Sea & $\mathrm{R}$ & M & Fine 1970 \\
\hline Dromidia antillensis & Sargasso Sea & $\mathrm{R}$ & $\mathrm{M}$ & Fine 1970 \\
\hline Garthiope anchialina & Galapagos & $\mathrm{D}$ & n.i. & Peck 1994 \\
\hline Geograpsus lividus & Galapagos & $\mathrm{D}$ & n.i. & Peck 1994 \\
\hline Grapsus grapsus & Galapagos & $\mathrm{D}$ & n.i. & Peck 1994 \\
\hline Grapsus sp. & Costa Rica & $\mathrm{R}$ & $\mathrm{O}$ & Wehrtmann \& Dittel 1990 \\
\hline Guitonia troglophila & Galapagos & $\mathrm{D}$ & n.i. & Peck 1994 \\
\hline Halicarcinus ovatus & Tasmania & $\mathrm{R}$ & $\mathrm{M}$ & Edgar 1987 \\
\hline Hemigrapsus penicillatus & Sagami Bay, Japan & $\mathrm{R}$ & M & Hirosaki 1964 \\
\hline Hyas araneus & off Texel & $\mathrm{R}$ & M & Van der Baan et al. 1972 \\
\hline Liocarcinus corrugatus & Irish Sea & $\mathrm{R}$ & M & Davenport \& Rees 1993 \\
\hline Liocarcinus depurator & Irish Sea & $\mathrm{R}$ & M & Davenport \& Rees 1993 \\
\hline Liocarcinus pusillus & Irish Sea & $\mathrm{R}$ & M & Davenport \& Rees 1993 \\
\hline Litocheira bispinosa & Tasmania & $\mathrm{R}$ & M & Edgar 1987 \\
\hline Macropodia rostrata & off Texel & $\mathrm{R}$ & M & Van der Baan et al. 1972 \\
\hline Macropodia tenuirostris & off Texel & $\mathrm{R}$ & M & Van der Baan et al. 1972 \\
\hline Necora puber & Irish Sea & $\mathrm{R}$ & M & Davenport \& Rees 1993 \\
\hline Neptunus sayi & NW-Atlantic, Red Sea & $\mathrm{R}$ & M & $\begin{array}{l}\text { Adams 1960, Markkaveeva } \\
1965\end{array}$ \\
\hline Ocypode gaudichaudii & Galapagos & $\mathrm{D}$ & n.i. & Peck 1994 \\
\hline Ovalipes guadulpensis & Sargasso Sea & $\mathrm{R}$ & M & Fine 1970 \\
\hline Pachygrapsus crassipes & California & $\mathrm{R}$ & M & $\begin{array}{l}\text { Shanks } 1985 \text {, Bushing } 1994, \\
\text { Hobday } 2000 \text { a }\end{array}$ \\
\hline Pachygrapsus marinus & New Zealand & $\mathrm{B}$ & $\mathrm{O}$ & Dell 1964 \\
\hline Pachygrapsus transversus & Panama & $\mathrm{R}$ & $\mathrm{W}$ & Donlan \& Nelson 2003 \\
\hline Paraxanthias taylori & California & $\mathrm{R}$ & M & Hobday 2000a \\
\hline Pinnixa sp. & California & $\mathrm{R}$ & M & Bushing 1994 \\
\hline Pinnixa sp. & Costa Rica & $\mathrm{R}$ & $\mathrm{O}$ & Wehrtmann \& Dittel 1990 \\
\hline
\end{tabular}




\section{MARTIN THIEL \& LARS GUTOW}

Table 9 (continued) Arthropoda Malacostraca reported or inferred as rafting

\begin{tabular}{|c|c|c|c|c|}
\hline Species & Region & $\begin{array}{l}\text { Rafting } \\
\text { inference }\end{array}$ & $\begin{array}{l}\text { Floating } \\
\text { substratum }\end{array}$ & Reference \\
\hline Pinnotheres sp. & Costa Rica & $\mathrm{R}$ & $\mathrm{O}$ & Wehrtmann \& Dittel 1990 \\
\hline Pisidia longicornis & Irish Sea & $\mathrm{R}$ & M & Davenport \& Rees 1993 \\
\hline Plagusia dentipes & Sagami Bay, Japan & $\mathrm{R}$ & M & Hirosaki 1964 \\
\hline Plagusia depressa & & $\mathrm{R}$ & $\mathrm{O}$ & Schubart et al. 2001 \\
\hline Plagusia immaculata & Panama & $\mathrm{R}$ & $\mathrm{W}$ & Donlan \& Nelson 2003 \\
\hline Plagusia sp. & off Costa Rica & $\mathrm{R}$ & W & Hunter \& Mitchell 1967 \\
\hline Planes cyaneus & $\begin{array}{l}\text { Panama, Sagami Bay, } \\
\text { Japan, California, Texas }\end{array}$ & $\mathrm{B}, \mathrm{R}$ & $\mathrm{M}, \mathrm{W}$ & $\begin{array}{l}\text { Hirosaki 1964, Shirley } 1974, \\
\text { Hobday 2000a, Donlan \& } \\
\text { Nelson 2003, Sano et al. } 2003\end{array}$ \\
\hline Planes marinus & S-Chile, Argentina & $\mathrm{B}, \mathrm{R}$ & $\mathrm{O}, \mathrm{P}$ & $\begin{array}{l}\text { Jara \& Jaramillo 1979, Spivak } \\
\text { \& Bas } 1999\end{array}$ \\
\hline Planes minutus & $\begin{array}{l}\text { N-Atlantic, Sargasso } \\
\text { Sea, Madeira, } \\
\text { Bermuda, British } \\
\text { Channel, Red Sea, } \\
\text { Azores, Gulf Stream }\end{array}$ & $\mathrm{R}$ & $\mathrm{M}, \mathrm{P}, \mathrm{W}$ & $\begin{array}{l}\text { Goodsir 1845*, Hodgson } \\
\text { 1896*, Crozier 1918, } \\
\text { Hentschel 1922, Hitchcock } \\
\text { 1941, Adams 1960, } \\
\text { Markkaveeva 1965, Fine } \\
\text { 1970, Burns \& Teal 1973, } \\
\text { Butler et al. 1983, Stoner \& } \\
\text { Greening 1984, Coston- } \\
\text { Clements et al. 1991, Hacker } \\
\text { \& Madin 1991, Dellinger et al. } \\
\text { 1997, Morton \& Britton } 2000\end{array}$ \\
\hline Portunus anceps & Sargasso Sea & $\mathrm{R}$ & M & Fine 1970 \\
\hline Portunus asper & Panama & $\mathrm{R}$ & $\mathrm{W}$ & Donlan \& Nelson 2003 \\
\hline Portunus gladiator & Sagami Bay, Japan & $\mathrm{R}$ & M & Hirosaki 1964 \\
\hline Portunus ordwayi & Sargasso Sea & $\mathrm{R}$ & M & Fine 1970 \\
\hline Portunus sanguinolentus & Sagami Bay, Japan & $\mathrm{R}$ & M & Hirosaki 1964 \\
\hline Portunus sayi & $\begin{array}{l}\text { Sargasso Sea, Azores, } \\
\text { Gulf Stream, N- } \\
\text { Atlantic }\end{array}$ & $\mathrm{R}$ & M & $\begin{array}{l}\text { Adams 1960, Markkaveeva } \\
\text { 1965, Fine 1970, Burns \& } \\
\text { Teal 1973, Butler et al. 1983, } \\
\text { Stoner \& Greening 1984, } \\
\text { Coston-Clements et al. 1991, } \\
\text { Morton \& Britton } 2000\end{array}$ \\
\hline Portunus spinimanus & Sargasso Sea & $\mathrm{R}$ & M & $\begin{array}{l}\text { Butler et al. 1983, Coston- } \\
\text { Clements et al. } 1991\end{array}$ \\
\hline Portunus trituberculatus & $\begin{array}{l}\text { Sagami Bay, Seto Inland } \\
\text { Sea, Japan }\end{array}$ & $\mathrm{R}$ & M & Hirosaki 1964, Tanigawa 2001 \\
\hline Portunus sp. & $\mathrm{N}$-Atlantic & $\mathrm{R}$ & M & Johnson \& Braman 1975 \\
\hline Pugettia dalli & California & $\mathrm{R}$ & M & Hobday $2000 \mathrm{a}$ \\
\hline Pugettia producta & California & $\mathrm{R}$ & M & Bushing 1994, Hobday 2000a \\
\hline Taliepus nuttalli & California & $\mathrm{R}$ & M & Hobday 2000a \\
\hline Telmessus cheiragonus & Japan & $\mathrm{R}$ & M & Sano et al. 2003 \\
\hline Thalamita admete & Red Sea & $\mathrm{R}$ & M & Markkaveeva 1965 \\
\hline Thalamita picta & Sagami Bay, Japan & $\mathrm{R}$ & M & Hirosaki 1964 \\
\hline Thalamita stimpsoni & Great Barrier Reef & $\mathrm{R}$ & $\mathrm{O}$ & DeVantier 1992 \\
\hline Trapezia ferruginea & E-Pacific & G & M & Scheltema 1988 \\
\hline Uca galapagensis & Galapagos & $\mathrm{D}$ & n.i. & Peck 1994 \\
\hline Uca helleri & Galapagos & $\mathrm{D}$ & n.i. & Peck 1994 \\
\hline Uca sp. & Costa Rica & $\mathrm{R}$ & $\mathrm{O}$ & Wehrtmann \& Dittel 1990 \\
\hline
\end{tabular}




\section{RAFTING OF BENTHIC MARINE ORGANISMS}

Table 9 (continued) Arthropoda Malacostraca reported or inferred as rafting

\begin{tabular}{|c|c|c|c|c|}
\hline Species & Region & $\begin{array}{l}\text { Rafting } \\
\text { inference }\end{array}$ & $\begin{array}{l}\text { Floating } \\
\text { substratum }\end{array}$ & Reference \\
\hline \multicolumn{5}{|l|}{ Decapoda, Caridea } \\
\hline Alpheus clamator & California & $\mathrm{R}$ & M & Hobday 2000a \\
\hline Alpheus parasocialis & Tasmania & $\mathrm{R}$ & M & Edgar 1987 \\
\hline Alpheus sp. & $\begin{array}{l}\text { NW-Atlantic, Sargasso } \\
\text { Sea }\end{array}$ & $\mathrm{R}$ & M & $\begin{array}{l}\text { Adams } 1960 \text {, Coston-Clements } \\
\text { et al. } 1991\end{array}$ \\
\hline Ambidexter panamense & Costa Rica & $\mathrm{R}$ & $\mathrm{O}$ & Wehrtmann \& Dittel 1990 \\
\hline Atya $\mathrm{sp}$ & Costa Rica & $\mathrm{R}$ & $\mathrm{O}$ & Wehrtmann \& Dittel 1990 \\
\hline Heptacarpus geniculatus & Sagami Bay, Japan & $\mathrm{R}$ & M & Hirosaki 1964 \\
\hline Heptocarpus spp. & California & $\mathrm{R}$ & M & Hobday 2000a \\
\hline Hippolyte bifidirostris & New Zealand & $\mathrm{R}$ & M & Kingsford \& Choat 1985 \\
\hline Hippolyte californiensis & California & $\mathrm{R}$ & M & Hobday 2000a \\
\hline Hippolyte clarki & California & $\mathrm{R}$ & M & Hobday 2000a \\
\hline Hippolyte coerulescens & $\begin{array}{l}\text { Sargasso Sea, Bermuda, } \\
\text { Gulf Stream }\end{array}$ & $\mathrm{R}$ & M & $\begin{array}{l}\text { Butler et al. 1983, Stoner \& } \\
\text { Greening 1984, Coston- } \\
\text { Clements et al. 1991, Hacker } \\
\text { \& Madin } 1991\end{array}$ \\
\hline Hippolyte zostericola & Sargasso Sea & $\mathrm{R}$ & M & $\begin{array}{l}\text { Butler et al. 1983, Coston- } \\
\text { Clements et al. } 1991\end{array}$ \\
\hline Latreutes acicularis & Sagami Bay, Japan & $\mathrm{R}$ & M & Hirosaki 1964 \\
\hline Latreutes fucorum & $\begin{array}{l}\text { NW-Atlantic, Sargasso } \\
\text { Sea, Bermuda, Red Sea }\end{array}$ & $\mathrm{R}$ & M & $\begin{array}{l}\text { Adams } 1960 * \text {, Markkaveeva } \\
\text { 1965*, Fine 1970, Butler et al. } \\
\text { 1983, Stoner \& Greening } \\
\text { 1984, Coston-Clements et al. } \\
\text { 1991*, Hacker \& Madin } 1991\end{array}$ \\
\hline Latreutes laminirostris & Sagami Bay, Japan & $\mathrm{R}$ & M & Hirosaki 1964 \\
\hline Leander tenuicornis & $\begin{array}{l}\text { NW-Atlantic, Sargasso } \\
\text { Sea, Bermuda, Red Sea, } \\
\text { Gulf Stream }\end{array}$ & $\mathrm{R}$ & M & $\begin{array}{l}\text { Goodsir } 1845^{*} \text {, Adams } 1960, \\
\text { Markkaveeva 1965, Fine } \\
\text { 1970, Burns \& Teal 1973, } \\
\text { Butler et al. 1983, Stoner \& } \\
\text { Greening 1984, Coston- } \\
\text { Clements et al. 1991, Hacker } \\
\text { \& Madin } 1991\end{array}$ \\
\hline Lysmata californica & California & $\mathrm{R}$ & M & Hobday 2000a \\
\hline Macrobrachium sp. & Costa Rica & $\mathrm{R}$ & $\mathrm{O}$ & Wehrtmann \& Dittel 1990 \\
\hline Palaemon affinis & New Zealand & $\mathrm{R}$ & M & Kingsford \& Choat 1985 \\
\hline Palaemonella gracilis & Red Sea & $\mathrm{R}$ & M & Markkaveeva 1965 \\
\hline Palaemonetes schmitti & Costa Rica & $\mathrm{R}$ & $\mathrm{O}$ & Wehrtmann \& Dittel 1990 \\
\hline Synalpheus lockingtoni & California & $\mathrm{R}$ & M & Hobday $2000 \mathrm{a}$ \\
\hline Tozeuma carolinense & Sargasso Sea & $\mathrm{R}$ & M & $\begin{array}{l}\text { Butler et al. 1983, Coston- } \\
\text { Clements et al. } 1991\end{array}$ \\
\hline \multicolumn{5}{|l|}{ Decapoda, Penaeoidea } \\
\hline Lucifer faxoni & Sargasso Sea & $\mathrm{R}$ & M & Fine 1970 \\
\hline Penaeus californiensis & Costa Rica & $\mathrm{R}$ & $\mathrm{O}$ & Wehrtmann \& Dittel 1990 \\
\hline Penaeus occidentalis & Costa Rica & $\mathrm{R}$ & $\mathrm{O}$ & Wehrtmann \& Dittel 1990 \\
\hline Penaeus stylirostris & Costa Rica & $\mathrm{R}$ & $\mathrm{O}$ & Wehrtmann \& Dittel 1990 \\
\hline Penaeus vannamei & Costa Rica & $\mathrm{R}$ & $\mathrm{O}$ & Wehrtmann \& Dittel 1990 \\
\hline Penaeus sp. & Costa Rica & $\mathrm{R}$ & $\mathrm{O}$ & Wehrtmann \& Dittel 1990 \\
\hline
\end{tabular}




\section{MARTIN THIEL \& LARS GUTOW}

Table 9 (continued) Arthropoda Malacostraca reported or inferred as rafting

\begin{tabular}{|c|c|c|c|c|}
\hline Species & Region & $\begin{array}{l}\text { Rafting } \\
\text { inference }\end{array}$ & $\begin{array}{l}\text { Floating } \\
\text { substratum }\end{array}$ & Reference \\
\hline \multicolumn{5}{|l|}{ Amphipoda } \\
\hline Aciconula sp. & China Sea & $\mathrm{C}$ & n.i. & Takeuchi \& Sawamoto 1998 \\
\hline Allorchestes angusta & Japan & $\mathrm{R}$ & M & Sano et al. 2003 \\
\hline Allorchestes sp. & Sargasso Sea & $\mathrm{R}$ & M & Fine 1970 \\
\hline Ampelisca euroa & Tasmania & $\mathrm{R}$ & M & Edgar 1987 \\
\hline Amphithoe rubricata & Iceland & $\mathrm{R}$ & M & Ingólfsson 1995 \\
\hline Amphithoe vaillanti & Red Sea & $\mathrm{R}$ & M & Markkaveeva 1965 \\
\hline Ampilochus sp. & Red Sea & $\mathrm{R}$ & M & Markkaveeva 1965 \\
\hline Ampithoe lacertosa & Japan, NW coast of USA & $\mathrm{R}$ & M & $\begin{array}{l}\text { Highsmith 1985, Sano et al. } \\
2003\end{array}$ \\
\hline Ampithoe longimana & Sargasso Sea & $\mathrm{R}$ & M & $\begin{array}{l}\text { Fine } 1970, \text { Butler et al. } 1983, \\
\text { Coston-Clements et al. } 1991\end{array}$ \\
\hline Ampithoe sp. & Tasmania & $\mathrm{R}$ & M & Edgar 1987 \\
\hline Aora hircosa & Tasmania & $\mathrm{R}$ & M & Edgar 1987 \\
\hline Aora maculata & Tasmania & $\mathrm{R}$ & M & Edgar 1987 \\
\hline Aora typica & Red Sea & $\mathrm{R}$ & M & Markkaveeva 1965 \\
\hline Aoroides sp. & NW coast of USA & $\mathrm{R}$ & M & Highsmith 1985 \\
\hline Apherusa ovalipes & German Bight & $\mathrm{R}$ & M & Franke \& Gutow 2004 \\
\hline Atylus guttatus & German Bight & $\mathrm{R}$ & M & Franke \& Gutow 2004 \\
\hline Atylus minikoi & Sargasso Sea & $\mathrm{R}$ & M & Fine 1970 \\
\hline Biancolina brassicacephala & $\begin{array}{l}\text { Sargasso Sea, Gulf } \\
\text { Stream }\end{array}$ & $\mathrm{R}$ & M & $\begin{array}{l}\text { Butler et al. 1983, Stoner \& } \\
\text { Greening 1984, Coston- } \\
\text { Clements et al. } 1991\end{array}$ \\
\hline Biancolina sp. & Sargasso Sea & $\mathrm{R}$ & M & Fine 1970 \\
\hline Brolgus tattersalli & Tasmania & $\mathrm{R}$ & M & Edgar 1987 \\
\hline Calliopius laeviusculus & $\begin{array}{l}\text { Iceland, Bay of Fundy, } \\
\text { N-Pacific, NW-Pacific }\end{array}$ & $\mathrm{R}$ & $\mathrm{M}, \mathrm{O}, \mathrm{P}$ & $\begin{array}{l}\text { Sinitsyn \& Reznichenko 1981, } \\
\text { Locke \& Corey 1989, } \\
\text { Ingólfsson 1995, 1998, 2000, } \\
\text { Tsikhon-Lukanina et al. } \\
\text { 2001a }\end{array}$ \\
\hline Calliopius sp. & Japan & $\mathrm{R}$ & M & Sano et al. 2003 \\
\hline Calliopius sp. & US West Coast & $\mathrm{R}$ & M & Shaffer et al. 1995 \\
\hline Caprella acanthogaster & Kuroshio & $\mathrm{R}$ & M & Safran \& Omori 1990 \\
\hline Caprella acutifrons & Sagami Bay, Japan & $\mathrm{R}$ & $\mathrm{M}$ & Hirosaki 1964 \\
\hline Caprella andreae & $\begin{array}{l}\text { East China Sea, } \\
\text { Argentina }\end{array}$ & $\mathrm{B}, \mathrm{R}$ & $\mathrm{P}, \mathrm{W}$ & $\begin{array}{l}\text { Aoki \& Kikuchi 1995, Spivak } \\
\text { \& Bas } 1999\end{array}$ \\
\hline Caprella californica & California, Japan & $\mathrm{R}$ & M & $\begin{array}{l}\text { Bushing 1994, Hobday 2000a, } \\
\text { Sano et al. } 2003\end{array}$ \\
\hline Caprella danilevskii & $\begin{array}{l}\text { Sargasso Sea, Sagamy } \\
\text { Bay, Japan }\end{array}$ & $\mathrm{R}$ & M & $\begin{array}{l}\text { Hirosaki 1964, Butler et al. } \\
\text { 1983, Coston-Clements et al. } \\
\text { 1991, Sano et al. } 2003\end{array}$ \\
\hline Caprella decipiens & Sagami Bay, Japan & $\mathrm{R}$ & M & Hirosaki 1964 \\
\hline Caprella drepanochir & Sagami Bay, Japan & $\mathrm{R}$ & M & Hirosaki 1964 \\
\hline Caprella equilibra & $\begin{array}{l}\text { Sagami Bay, Japan, } \\
\text { China Sea, Chile coast }\end{array}$ & $\mathrm{C}, \mathrm{R}$ & $\mathrm{M}, \mathrm{O}$ & $\begin{array}{l}\text { Hirosaki } 1964, \text { Takeuchi \& } \\
\text { Sawamoto 1998, Sano et al. } \\
\text { 2003, Thiel et al. } 2003\end{array}$ \\
\hline Caprella linearis & $\begin{array}{l}\text { Azores, Sagamy Bay, } \\
\text { Japan }\end{array}$ & $\mathrm{C}, \mathrm{R}$ & $\mathrm{M}, \mathrm{O}$ & $\begin{array}{l}\text { Hirosaki 1964, Morton \& } \\
\text { Britton } 2000\end{array}$ \\
\hline Caprella mutica & Japan & $\mathrm{R}$ & M & Sano et al. 2003 \\
\hline
\end{tabular}




\section{RAFTING OF BENTHIC MARINE ORGANISMS}

Table 9 (continued) Arthropoda Malacostraca reported or inferred as rafting

\begin{tabular}{|c|c|c|c|c|}
\hline Species & Region & $\begin{array}{l}\text { Rafting } \\
\text { inference }\end{array}$ & $\begin{array}{l}\text { Floating } \\
\text { substratum }\end{array}$ & Reference \\
\hline Caprella obtusifrons & Sagami Bay, Japan & $\mathrm{R}$ & M & Hirosaki 1964 \\
\hline Caprella penantis & Japan, North Sea & $\mathrm{B}, \mathrm{R}$ & M & $\begin{array}{l}\text { Sano et al. 2003, Sokolowsky } \\
1912^{*}\end{array}$ \\
\hline Caprella scaura & $\begin{array}{l}\text { Sagami Bay, Japan, } \\
\text { Chile coast }\end{array}$ & $\mathrm{R}$ & $\mathrm{M}, \mathrm{O}$ & $\begin{array}{l}\text { Hirosaki 1964, Sano et al. } \\
\text { 2003, Thiel et al. } 2003\end{array}$ \\
\hline Caprella septentrionalis & Sagami Bay, Japan & $\mathrm{R}$ & M & Hirosaki 1964 \\
\hline Caprella subinermis & Sagami Bay, Japan & $\mathrm{R}$ & M & Hirosaki 1964 \\
\hline Caprella tsugarensis & Japan & $\mathrm{R}$ & M & Sano et al. 2003 \\
\hline Caprella tuberculata & North Sea & $\mathrm{B}$ & M & Sokolowsky 1912 \\
\hline Caprella verrucosa & Chile coast & $\mathrm{R}$ & $\mathrm{O}$ & Thiel et al. 2003 \\
\hline Ceradocus sellickiensis & Tasmania & $\mathrm{R}$ & M & Edgar 1987 \\
\hline Chelorchestia costaricana & Galapagos & $\mathrm{D}$ & n.i. & Peck 1994 \\
\hline Chelorchestia vaggala & Galapagos & $\mathrm{D}$ & n.i. & Peck 1994 \\
\hline Corophium acherusicum & German Bight & $\mathrm{R}$ & M & Franke \& Gutow 2004 \\
\hline Corophium sextoni & Azores & $\mathrm{C}$ & $\mathrm{O}$ & Morton \& Britton 2000 \\
\hline Cottesloe berringar & Tasmania & $\mathrm{R}$ & M & Edgar 1987 \\
\hline Deutella venenosa & Chile coast & $\mathrm{R}$ & $\mathrm{O}$ & Thiel et al. 2003 \\
\hline Dexamine thea & Iceland, German Bight & $\mathrm{R}$ & $\mathrm{M}, \mathrm{O}, \mathrm{P}$ & $\begin{array}{l}\text { Ingólfsson 1998, 2000, Franke } \\
\text { \& Gutow } 2004\end{array}$ \\
\hline Elasmopus rapax & German Bight & $\mathrm{R}$ & M & Franke \& Gutow 2004 \\
\hline Ericthonius brasiliensis & Azores & $\mathrm{C}$ & $\mathrm{O}$ & Morton \& Britton 2000 \\
\hline Ericthonius sp. & Tasmania & $\mathrm{R}$ & M & Edgar 1987 \\
\hline Gammarellus angulosus & Iceland & $\mathrm{R}$ & M & Ingólfsson 1995, 2000 \\
\hline Gammarellus homari & Iceland & $\mathrm{R}$ & M & Ingólfsson 1998 \\
\hline Gammaropsis haswelli & Tasmania & $\mathrm{R}$ & M & Edgar 1987 \\
\hline Gammaropsis thomsoni & Tasmania & $\mathrm{R}$ & M & Edgar 1987 \\
\hline Gammaropsis sp. & Japan & $\mathrm{R}$ & M & Sano et al. 2003 \\
\hline Gammarus locusta & $\begin{array}{l}\text { Irish Sea, Iceland, } \\
\text { Galway Bay }\end{array}$ & $\mathrm{R}$ & M & $\begin{array}{l}\text { Tully \& Ó Céidigh 1986a, } \\
\text { Davenport \& Rees 1993, } \\
\text { Ingólfsson } 1995,1998, \\
\text { Franke et al. } 1999\end{array}$ \\
\hline Gammarus obtusatus & Iceland & $\mathrm{R}$ & M & Ingólfsson 1995, 1998 \\
\hline Gammarus oceanicus & Iceland & $\mathrm{R}$ & M & Ingólfsson 1992, 1998 \\
\hline Gammarus wilkitzki & Iceland & $\mathrm{R}$ & M & Ingólfsson 1995 \\
\hline Gammarus sp. & Iceland & $\mathrm{R}$ & M & Ingólfsson 1998 \\
\hline Hemiaegina minuta & $\begin{array}{l}\text { Gulf Stream, Sargasso } \\
\text { Sea }\end{array}$ & $\mathrm{R}$ & M & $\begin{array}{l}\text { Fine } 1970 \text {, Butler et al. } 1983 \text {, } \\
\text { Stoner \& Greening } 1984, \\
\text { Coston-Clements et al. } 1991\end{array}$ \\
\hline Hyale hirtipalma & $\begin{array}{r}\text { Heard Island } \\
\text { (Kerguelen) }\end{array}$ & $\mathrm{R}$ & M & Edgar \& Burton 2000 \\
\hline Hyale nilssoni & Iceland & $\mathrm{R}$ & M & Ingólfsson 1998 \\
\hline Hyale pontica & Red Sea & $\mathrm{R}$ & M & Markkaveeva 1965 \\
\hline Hyale sp. & US West Coast & $\mathrm{R}$ & M & Shaffer et al. 1995 \\
\hline Hyperia galba & Sargasso Sea & $\mathrm{R}$ & M & Fine 1970 \\
\hline Hyperoche spp. & Japan & $\mathrm{R}$ & M & Sano et al. 2003 \\
\hline Ischyrocerus anguipes & Iceland & $\mathrm{R}$ & M & Ingólfsson 1998, 2000 \\
\hline Jassa falcata & Azores & $\mathrm{C}$ & $\mathrm{O}$ & Morton \& Britton 2000 \\
\hline Jassa marmorata & Japan & $\mathrm{R}$ & M & Sano et al. 2003 \\
\hline Jassa slatteryi & Japan & $\mathrm{R}$ & M & Sano et al. 2003 \\
\hline
\end{tabular}




\section{MARTIN THIEL \& LARS GUTOW}

Table 9 (continued) Arthropoda Malacostraca reported or inferred as rafting

\begin{tabular}{|c|c|c|c|c|}
\hline Species & Region & $\begin{array}{l}\text { Rafting } \\
\text { inference }\end{array}$ & $\begin{array}{l}\text { Floating } \\
\text { substratum }\end{array}$ & Reference \\
\hline Jassa sp. & Heard Island (Kerguelen) & $\mathrm{R}$ & M & Edgar \& Burton 2000 \\
\hline Lembos clematis & Tasmania & $\mathrm{R}$ & M & Edgar 1987 \\
\hline Leucothoe spinicarpa & Red Sea & $\mathrm{R}$ & M & Markkaveeva 1965 \\
\hline Liljeborgia pallida & German Bight & $\mathrm{R}$ & M & Franke \& Gutow 2004 \\
\hline Liljeborgia sp. & Japan & $\mathrm{R}$ & M & Sano et al. 2003 \\
\hline Luconacia incerta & $\begin{array}{l}\text { Gulf Stream, Sargasso } \\
\text { Sea }\end{array}$ & $\mathrm{R}$ & M & $\begin{array}{l}\text { Butler et al. 1983, Stoner \& } \\
\text { Greening 1984, Coston- } \\
\text { Clements et al. } 1991\end{array}$ \\
\hline Maera hamigera & Tasmania & $\mathrm{R}$ & M & Edgar 1987 \\
\hline Maera inaequipes & Red Sea & $\mathrm{R}$ & M & Markkaveeva 1965 \\
\hline Maera viridis & Tasmania & $\mathrm{R}$ & M & Edgar 1987 \\
\hline Mallacoota diemenensis & Tasmania & $\mathrm{R}$ & M & Edgar 1987 \\
\hline Orchestia montagui & Aegean Sea & G & M & De Matthaeis et al. 1998 \\
\hline Orchestia stephenseni & Aegean Sea & G & M & De Matthaeis et al. 2000 \\
\hline $\begin{array}{l}\text { Paracalliope } \\
\text { novizealandiae }\end{array}$ & New Zealand & $\mathrm{R}$ & $\mathrm{O}$ & Hicks 1988 \\
\hline Paracaprella cf. alarta & China Sea & $\mathrm{C}$ & n.i. & Takeuchi \& Sawamoto 1998 \\
\hline Paradexamine churinga & Tasmania & $\mathrm{R}$ & M & Edgar 1987 \\
\hline Paradexamine spp. & Japan & $\mathrm{R}$ & M & Sano et al. 2003 \\
\hline Parajassa pelagica & Iceland & $\mathrm{R}$ & M & Ingólfsson 1995 \\
\hline $\begin{array}{l}\text { Paramoera austrina } \mathrm{f} . \\
\text { kergueleni }\end{array}$ & $\begin{array}{r}\text { Heard Island } \\
\text { (Kerguelen) }\end{array}$ & $\mathrm{R}$ & M & Edgar \& Burton 2000 \\
\hline Paramphithoe lingbergi & Japan & $\mathrm{R}$ & M & Sano et al. 2003 \\
\hline Parawaldeckia yamba & Tasmania & $\mathrm{R}$ & M & Edgar 1987 \\
\hline Photis sp. & Tasmania & $\mathrm{R}$ & M & Edgar 1987 \\
\hline Phtisica marina & Mediterranean & $\mathrm{R}$ & $\mathrm{P}$ & Aliani \& Molcard 2003 \\
\hline Platorchestia platensis & Aegean Sea & G & M & De Matthaeis et al. 2000 \\
\hline Podocerus spp. & Japan & $\mathrm{R}$ & M & Sano et al. 2003 \\
\hline Pontogeneia spp. & Japan & $\mathrm{R}$ & M & Sano et al. 2003 \\
\hline Pontogenia sp. & NW coast of USA & $\mathrm{R}$ & M & Highsmith 1985 \\
\hline Protogeton cf. inflatus & China Sea & $\mathrm{C}$ & n.i. & Takeuchi \& Sawamoto 1998 \\
\hline Pseudoprotella phasma & Azores & $\mathrm{C}$ & $\mathrm{O}$ & Morton \& Britton 2000 \\
\hline Stenothoe valida & German Bight & $\mathrm{R}$ & M & Franke \& Gutow 2004 \\
\hline Stenothoe sp. & Japan & $\mathrm{R}$ & M & Sano et al. 2003 \\
\hline Stenothoe sp. & Red Sea & $\mathrm{R}$ & M & Markkaveeva 1965 \\
\hline Sunamphithoe pelagica & $\begin{array}{l}\text { NW-Atlantic, Bermuda, } \\
\text { Gulf Stream, Sargasso } \\
\text { Sea }\end{array}$ & $\mathrm{R}$ & M & $\begin{array}{l}\text { Goodsir } 1845^{*} \text {, Adams 1960*, } \\
\text { Fine 1970, Butler et al. 1983, } \\
\text { Stoner \& Greening 1984, } \\
\text { Coston-Clements et al. 1991*, } \\
\text { Hacker \& Madin } 1991\end{array}$ \\
\hline Talorchestia fritzi & Galapagos & $\mathrm{D}$ & n.i. & Peck 1994 \\
\hline Tethygenia sp. & Tasmania & $\mathrm{R}$ & M & Edgar 1987 \\
\hline Xenocheira fasciata & Tasmania & $\mathrm{R}$ & M & Edgar 1987 \\
\hline \multicolumn{5}{|l|}{ Isopoda } \\
\hline Bagatus minutus & $\begin{array}{l}\text { NW-Atlantic, Sargasso } \\
\text { Sea, Gulf Stream }\end{array}$ & $\mathrm{R}$ & M & $\begin{array}{l}\text { Adams } 1960^{*} \text {, Fine } 1970^{*}, \\
\text { Butler et al. 1983, Stoner \& } \\
\text { Greening 1984, Coston- } \\
\text { Clements et al. 1991* }\end{array}$ \\
\hline
\end{tabular}




\section{RAFTING OF BENTHIC MARINE ORGANISMS}

Table 9 (continued) Arthropoda Malacostraca reported or inferred as rafting

\begin{tabular}{|c|c|c|c|c|}
\hline Species & Region & $\begin{array}{l}\text { Rafting } \\
\text { inference }\end{array}$ & $\begin{array}{l}\text { Floating } \\
\text { substratum }\end{array}$ & Reference \\
\hline Bopyrus squillarum & $\begin{array}{l}\text { NW-Atlantic, Sargasso } \\
\text { Sea }\end{array}$ & $\mathrm{R}$ & M & $\begin{array}{l}\text { Goodsir 1845, Adams 1960, } \\
\text { Coston-Clements et al. } 1991\end{array}$ \\
\hline Caecijaera borealis & Iceland & $\mathrm{D}$ & W & Svavarsson 1982 \\
\hline Carpias bermudensis & Bermuda & $\mathrm{B}, \mathrm{R}$ & $\mathrm{M}, \mathrm{S}$ & Miller 1968, Gerlach 1977* \\
\hline Cubaris sp. & Puerto Rico & $\mathrm{R}$ & $\mathrm{O}$ & Heatwole \& Levins 1972 \\
\hline Cymodoce japonica & Japan & $\mathrm{R}$ & M & Sano et al. 2003 \\
\hline Eurydice inermis & Irish Sea & $\mathrm{R}$ & M & Davenport \& Rees 1993 \\
\hline Haswellia emarginata & Tasmania & $\mathrm{R}$ & M & Edgar 1987 \\
\hline Idotea baltica & $\begin{array}{l}\text { Sargasso Sea, Irish Sea, } \\
\text { German Bight, North } \\
\text { Sea, Mediterranean, } \\
\text { Iceland, Bay of Fundy, } \\
\text { Galway bay, Ireland, N- } \\
\text { Atlantic, N- } \\
\text { America/Europe, } \\
\text { Bermuda }\end{array}$ & $\mathrm{G}, \mathrm{R}$ & $\mathrm{M}, \mathrm{O}, \mathrm{P}, \mathrm{T}$ & $\begin{array}{l}\text { Vallentin 1895*, Miller 1968, } \\
\text { Butler et al. 1983, Holdway \& } \\
\text { Maddock 1983a, b, Tully \& } \\
\text { Ó Céidigh 1986a, Locke \& } \\
\text { Corey 1989, Coston-Clements } \\
\text { et al. 1991, Davenport \& Rees } \\
\text { 1993, Ingólfsson 1995, 1998, } \\
\text { 2000, Franke \& Janke 1998, } \\
\text { Franke et al. 1999, Gutow \& } \\
\text { Franke 2001, Wares 2001, } \\
\text { Wares \& Cunningham 2001, } \\
\text { Gutow \& Franke 2003, } \\
\text { Franke \& Gutow 2004 }\end{array}$ \\
\hline Idotea chelipes & Irish Sea, North Sea & $\mathrm{R}$ & M & $\begin{array}{l}\text { Davenport \& Rees } 1993^{*}, \\
\text { Franke et al. 1999, Gutow \& } \\
\text { Franke } 2003\end{array}$ \\
\hline Idotea emarginata & $\begin{array}{l}\text { Irish Sea, North Sea, } \\
\text { Iceland, Galway Bay, } \\
\text { Ireland }\end{array}$ & $\mathrm{R}$ & M & $\begin{array}{l}\text { Tully \& Ó Céidigh 1986a, } \\
\text { Davenport \& Rees 1993, } \\
\text { Ingólfsson 1995, Franke et al. } \\
\text { 1999, Gutow \& Franke } 2003\end{array}$ \\
\hline Idotea fewkesi & California & $\mathrm{R}$ & M & Hobday $2000 \mathrm{a}$ \\
\hline Idotea granulosa & $\begin{array}{l}\text { Irish Sea, North Sea, } \\
\text { Iceland, Galway Bay, } \\
\text { Ireland }\end{array}$ & $\mathrm{R}$ & M & $\begin{array}{l}\text { Tully \& Ó Céidigh 1986a, } \\
\text { Davenport \& Rees 1993, } \\
\text { Ingólfsson 1995, 1998, Franke } \\
\text { et al. 1999, Gutow \& Franke } \\
2003\end{array}$ \\
\hline Idotea linearis & $\begin{array}{l}\text { Irish Sea, North Sea, } \\
\text { Iceland, Galway Bay, } \\
\text { Ireland }\end{array}$ & $\mathrm{R}$ & M & $\begin{array}{l}\text { Tully \& Ó Céidigh 1986a, } \\
\text { Davenport \& Rees 1993, } \\
\text { Franke et al. 1999, Gutow \& } \\
\text { Franke } 2003\end{array}$ \\
\hline Idotea metallica & $\begin{array}{l}\text { NW-Atlantic, } \\
\text { Mediterranean, } \\
\text { Sargasso Sea, Irish Sea, } \\
\text { North Sea, Rhodes to } \\
\text { Azores, Bay of Fundy, } \\
\text { Norway, Kuroshio, } \\
\text { Japan, Galway Bay, } \\
\text { Ireland, N-Pacific, } \\
\text { Bermuda }\end{array}$ & $\mathrm{D}, \mathrm{R}$ & $\mathrm{M}, \mathrm{O}, \mathrm{P}, \mathrm{T}$ & $\begin{array}{l}\text { Adams 1960, Miller 1968, Van } \\
\text { der Baan \& Holthuis 1969, } \\
\text { Horn et al. 1970, Pethon 1970, } \\
\text { Wong et al. 1974, Butler 1975, } \\
\text { Butler et al. 1983, Holdway \& } \\
\text { Maddock 1983a, b, Tully \& } \\
\text { Ó Céidigh 1986a, Locke \& } \\
\text { Corey 1989, Safran \& Omori } \\
\text { 1990, Coston-Clements et al. } \\
\text { 1991, Davenport \& Rees 1993, } \\
\text { Franke et al. 1999, Gutow \& }\end{array}$ \\
\hline
\end{tabular}




\section{MARTIN THIEL \& LARS GUTOW}

Table 9 (continued) Arthropoda Malacostraca reported or inferred as rafting

\begin{tabular}{|c|c|c|c|c|}
\hline Species & Region & $\begin{array}{l}\text { Rafting } \\
\text { inference }\end{array}$ & $\begin{array}{l}\text { Floating } \\
\text { substratum }\end{array}$ & Reference \\
\hline & & & & $\begin{array}{l}\text { Franke 2001, Aliani \& Molcard } \\
\text { 2003, Gutow 2003a, b, Gutow } \\
\text { \& Franke 2003, Sano et al. } \\
\text { 2003, Franke \& Gutow } 2004\end{array}$ \\
\hline Idotea montereyensis & California & $\mathrm{R}$ & M & Hobday 2000a \\
\hline Idotea neglecta & $\begin{array}{l}\text { Irish Sea, North Sea, } \\
\text { Iceland, Galway Bay, } \\
\text { Ireland }\end{array}$ & $\mathrm{R}$ & M & $\begin{array}{l}\text { Tully \& Ó Céidigh 1986a, } \\
\text { Davenport \& Rees 1993, } \\
\text { Ingólfsson 1995, Franke et al. } \\
\text { 1999, Gutow \& Franke } 2003\end{array}$ \\
\hline Idotea ochotensis & Japan & $\mathrm{R}$ & M & Sano et al. 2003 \\
\hline Idotea pelagica & $\begin{array}{l}\text { North Sea, Iceland, } \\
\text { Galway Bay, Ireland }\end{array}$ & $\mathrm{R}$ & M & $\begin{array}{l}\text { Tully \& Ó Céidigh 1986a, } \\
\text { Ingólfsson 1995, Franke et al. } \\
\text { 1999, Gutow \& Franke } 2003\end{array}$ \\
\hline Idotea resecata & California & $\mathrm{R}$ & M & Hobday $2000 \mathrm{a}$ \\
\hline Idotea whymperi & $\begin{array}{l}\text { NW-Atlantic, Sargasso } \\
\text { Sea }\end{array}$ & $\mathrm{R}$ & M & $\begin{array}{l}\text { Adams } 1960, \text { Coston-Clements } \\
\text { et al. } 1991\end{array}$ \\
\hline Idotea $\mathrm{sp}$. & Sargasso Sea & $\mathrm{R}$ & $\mathrm{P}$ & Carpenter \& Smith 1972 \\
\hline Idotea sp. & US West Coast & $\mathrm{R}$ & M & Shaffer et al. 1995 \\
\hline Jaera sp. & Iceland & $\mathrm{R}$ & M & Ingólfsson 1995, 1998 \\
\hline Janira sp. & Red Sea & $\mathrm{R}$ & M & Markkaveeva 1965 \\
\hline Limnoria borealis & Island & $\mathrm{D}$ & $\mathrm{W}$ & Svavarsson 1982 \\
\hline Limnoria carptora & $\begin{array}{r}\text { Heard Island } \\
\text { (Kerguelen) }\end{array}$ & $\mathrm{R}$ & M & Edgar \& Burton 2000 \\
\hline Limnoria chilensis & Chile & $\mathrm{R}$ & M & Thiel et al., unpublished data \\
\hline Paracerceis cordata & California & $\mathrm{R}$ & M & Hobday $2000 \mathrm{a}$ \\
\hline Paradynamene benjamensis & Sargasso Sea & $\mathrm{R}$ & M & $\begin{array}{l}\text { Butler et al. 1983, Coston- } \\
\text { Clements et al. } 1991\end{array}$ \\
\hline Philoscia sp. & Puerto Rico & $\mathrm{R}$ & $\mathrm{O}$ & Heatwole \& Levins 1972 \\
\hline Phycolimnoria algarum & California & $\mathrm{R}$ & M & Bushing 1994 \\
\hline Phycolimnoria nonsegnis & Tasmania & $\mathrm{R}$ & M & Edgar 1987 \\
\hline Phycolimnoria sp. & Tasmania & $\mathrm{R}$ & M & Edgar 1987 \\
\hline Probopyrinella latreuticola & $\begin{array}{l}\text { NW-Atlantic, Sargasso } \\
\text { Sea }\end{array}$ & $\mathrm{R}$ & M & $\begin{array}{l}\text { Adams } 1960^{*} \text {, Fine } 1970^{*}, \\
\text { Coston-Clements et al. } 1991^{*}\end{array}$ \\
\hline Sphaeroma terebrans & Australia, Florida & $\mathrm{B}, \mathrm{C}$ & $\mathrm{W}$ & $\begin{array}{l}\text { Miller 1968, Si et al. 2000, } \\
\text { Brooks } 2004\end{array}$ \\
\hline Stenetrium sp. & Tasmania & $\mathrm{R}$ & M & Edgar 1987 \\
\hline Synidotea harfordi & California & $\mathrm{R}$ & M & Hobday $2000 \mathrm{a}$ \\
\hline \multicolumn{5}{|l|}{ Tanaidacea } \\
\hline Anatanais normani & Sargasso Sea, Bermuda & $\mathrm{R}$ & M & $\begin{array}{c}\text { Greve 1974, Butler et al. } 1983, \\
\text { Coston-Clements et al. } 1991\end{array}$ \\
\hline Leptochelia dubia & $\begin{array}{l}\text { Sargasso Sea, NW coats } \\
\text { of USA }\end{array}$ & $\mathrm{R}$ & M & Fine 1970, Highsmith 1985 \\
\hline Leptochelia ignota & Tasmania & $\mathrm{R}$ & M & Edgar 1987 \\
\hline Paratanais sp. & Tasmania & $\mathrm{R}$ & M & Edgar 1987 \\
\hline
\end{tabular}




\section{RAFTING OF BENTHIC MARINE ORGANISMS}

Table 9 (continued) Arthropoda Malacostraca reported or inferred as rafting

\begin{tabular}{llcll}
\hline Species & Region & $\begin{array}{l}\text { Rafting } \\
\text { inference }\end{array}$ & $\begin{array}{l}\text { Floating } \\
\text { substratum }\end{array}$ & Reference \\
\hline Tanais dulongii & Azores & $\mathrm{C}$ & $\mathrm{M}$ & Morton \& Britton 2000 \\
Tanais stanfordi & Galapagos & $\mathrm{D}$ & n.i. & Peck 1994 \\
\hline
\end{tabular}

Notes: $\mathrm{B}=$ stranded floating item on beach; $\mathrm{C}=$ circumstantial; $\mathrm{D}=$ distributional inference; $\mathrm{G}=$ genetic inference; $\mathrm{M}=$ macroalgae; $\mathrm{n} . \mathrm{i} .=$ no information; $\mathrm{O}=$ other; $\mathrm{P}=$ plastics; $\mathrm{R}=$ rafting; $\mathrm{S}=$ seeds; $\mathrm{T}=\operatorname{tar}$ lumps; $\mathrm{W}=$ wood.

* References refer to this species under a different name.

(Davenport \& Rees 1993, M. Thiel, personal observation). Also juvenile portunid crabs may occur in high abundances on floating macroalgae (Tanigawa 2001).

Adults from the genus Plagusia can frequently be found on floating items (Schubart et al. 2001). The species $P$. depressa and P. squamosa from the Atlantic and Indo-Pacific, respectively, are very similar in morphology and only recent molecular studies revealed that they are two distinct species (Schubart et al. 2001). The authors concluded that despite the high dispersal capabilities of these species, exchange between the Indo-Pacific and Atlantic oceans around the southern tips of the major continents has not occurred.

Some decapods are obligate rafters. Species from the genus Planes colonise a variety of floating objects ranging from floating Sargassum (Hitchcock 1941, Sano et al. 2003) over tree trunks and buoys (Chace 1966, Jara \& Jaramillo 1979) to sea turtles (Dellinger et al. 1997). Davenport (1992) reported high abundances of Planes minutus on Sargassum rafts. Population densities of Planes minutus are linked to the quantity of floating substrata because this crab has never been collected from waters free of floating items. The fact that crabs on wood and other flotsam are usually small and non-reproductive suggests that these items represent secondary habitats and that turtles are the preferred habitat of these crabs. Another obligate rafter is Pachygrapsus marinus. This species is found on flotsam in the Pacific (Chace 1951). It has been found among a dense growth of stalked barnacles (Lepas sp.) on a large glass fishing float (Dell 1964). Van der Baan et al. (1972) explained the occurrence of Macropodia sp. and Hyas araneus in the neuston off the Dutch coast with an association of the animals with floating seaweed accumulating at the hull of a lightship. On the Great Barrier Reef crabs of the species Thalamita stimpsoni have been found rafting on buoyant corals (DeVantier 1992).

Genetic distance between Hawaiian and Panamanian populations of the brachyuran crab Trapezia ferruginea did not differ significantly from zero (Huber 1985) indicating either that the eastern population had recently immigrated from the central Pacific, or that there exists gene flow between the two regions. Long-distance dispersal between the two locations was considered impossible because the duration of larval development of this species is too short. Even though the author does not mention the possibility of population connection by rafting, this mechanism is not unlikely because Trapezia ferruginea is a common symbiont of corals from the genus Pocillopora, which have repeatedly been observed rafting (Jokiel 1984).

Caridean and peneid shrimp are also frequently found on floating macroalgae (Hirosaki 1964, Kingsford \& Choat 1985, Hobday 2000a). Some species from the Sargassum community spend their entire life cycle on floating algae (Butler et al. 1983, Hacker \& Madin 1991). Most species are grazers that feed on their floating substratum. While marine decapods are frequently found on floating items, Marcus (1926) and Wittmann (1934) precluded large-scale dispersal of freshwater crabs over entire oceans for physiological reasons. 


\section{MARTIN THIEL \& LARS GUTOW}

\section{Arthropoda (Crustacea, Peracarida)}

Amphipods, isopods and tanaids have been reported from a wide variety of floating items, and these groups often dominate the rafting fauna (e.g., Sano et al. 2003) (Table 9). On macroalgae collected off southern California, peracarids (represented by amphipods and isopods) were found in about $90 \%$ of all samples (Bushing 1994). Davenport \& Rees (1993) mentioned a striking dominance of intertidal isopods of the genus Idotea and fairly high numbers of the amphipod Gammarus locusta on floating weed patches in the Irish Sea. Amphipods of many different species were the most diverse and most abundant group on detached Macrocystis pyrifera in Tasmanian waters (Edgar 1987). Even several months after detachment of the plants, amphipods were found in high numbers on the holdfasts of the algae, indicating that these species are well adapted to rafting conditions. Furthermore, isopods and tanaids, too, inhabited the floating plants throughout the whole experiment, which lasted $>6$ months. Miller (1968) suggested that some isopod and tanaid species may have colonised buoys in coastal waters of North America via rafting. Peracarids floating at the surface of the sea have a great capability to attach or cling to floating substrata if they make contact with them. Highsmith (1985) observed tanaids of the species Leptochelia dubia floating at the water surface in experimental containers for more than 5 days due to the hydrophobic nature of their exoskeleton. Hydrophobia is not an intrinsic characteristic of the cuticle but is maintained actively. All animals that resubmerged from the surface after floating were alive and built tubes at the bottom of the containers indicating that autonomous floating can also result in dispersal. Some species, e.g. the amphipod Calliopius laeviusculus, have also been collected in surface waters when no floating algae were present, but abundances were much higher in samples containing algae or seagrasses (Locke \& Corey 1989). Calliopius sp. was also the most abundant species on floating macroalgae in the San Juan Archipelago, Washington, U.S. (Shaffer et al. 1995). Densities of this amphipod were significantly higher in floating vegetation than in the surrounding water. Calliopius sp. is an important prey species for the splitnose rockfish Sebastes diplopora that feeds intensively on the rafting community (Shaffer et al. 1995).

Caprellid amphipods had been reported from floating macroalgae (Anraku \& Azeta 1967, Hobday 2000a) and other floating items, e.g., buoys (Aliani \& Molcard 2003, Thiel et al. 2003). Takeuchi \& Sawamoto (1998) reported many caprellids from plankton samples in the western North Pacific, and they implied that at least some of these could have reached these offshore waters clinging to floating items.

Amphipods and isopods also represent an important element of the Sargassum community (Butler et al. 1983, Sano et al. 2003). Investigating the amphipod fauna on benthic Sargassum at the Brazilian coast Wakabara et al. (1983) described the shrublike structure of Sargassum as beneficial for the motile mode of life of many amphipods because it efficiently provides protection from physical stress under exposed conditions.

The isopods Idotea baltica and I. metallica were almost exclusively limited to samples containing algae or seagrass blades (Locke \& Corey 1989) indicating the importance of floating substrata for these species. Idotea metallica is reported from many different substrata, including algae (Locke \& Corey 1989, Aliani \& Molcard 2003, Sano et al. 2003), plastics (Gutow \& Franke 2003), and even tar balls (Horn et al. 1970, Wong et al. 1974, Butler 1975) and it is an obligate rafter that lives entirely on floating items. This species is considered cosmopolitan (Van der Baan \& Holthuis 1969). Idotea baltica, in contrast, is a facultative rafter (Locke \& Corey 1989), which is presumably carried away from the shore on floating macroalgae (Davenport \& Rees 1993). It is commonly reported from floating algae (e.g., Richardson 1905) but it is known that floating algae represent a suboptimal habitat for I. baltica (Franke \& Janke 1998). The preferred habitat of this species is decaying macroalgal assemblages at the sea floor in shallow coastal waters. There, however, it is confronted with the congeneric competitor I. emarginata. Competitive inferiority 


\section{RAFTING OF BENTHIC MARINE ORGANISMS}

causes I. baltica to leave the preferred benthic habitat in favour of floating algae that provide temporally limited food supply and less protection from pelagic predators. Finding several Idotea species together on floating algae in Galway Bay (western Ireland) led Tully \& Ó Céidigh (1986a) to conclude that floating algae represent only a temporary and unstable habitat for these animals. Strong competitive interactions would not allow for a long-term coexistence of ecologically very similar species. Ingólfsson (1995) also considered I. baltica as a rather benthic species that colonises macroalgae when they are already afloat. Idotea baltica exhibits an amphi-Atlantic distribution with specimens from North America being genetically identical to specimens from Europe (Wares 2001). Rafting has been inferred as an important dispersal mechanism for this species and a potential source of genetic transfer between intertidal populations (Davenport \& Rees 1993).

The amphipod Gammarellus angulosus around Iceland is generally regarded as an inhabitant of shallow subtidal habitats. It is known to cling to algae of highly exposed rocky shores (Steele \& Steele 1972) but had never been recorded from floating macroalgae before 1995. In Iceland G. angulosus is rare in the intertidal (Ingólfsson 1977) but on algal rafts from that region it is by far the most common amphipod species occurring in samples collected at various distances up to more than $60 \mathrm{~km}$ from the coast (Ingólfsson 1995).

Davenport \& Rees (1993) also reported several individuals of the sand-dwelling isopod Eurydice inermis from floating macroalgae, pointing out that it was unexpected to find this species as a rafter. Eurydice inermis is known to migrate to the neuston during night-time (Tully \& Ó Céidigh 1986b) and thus the occurrence of this species on the algae might be the result of an incidental encounter with floating plants in surface waters. Since Davenport \& Rees (1993) took their samples in July, the probability of finding $E$. inermis at the surface was quite high because the abundance of this species in the neuston correlates positively with temperature, with highest densities during summer (Tully \& Ó Céidigh 1986b).

Distributional evidence for rafting has also been brought forward for many other peracarid species. Svavarsson (1982) suggested that the wood-dwelling isopod Caecijaera borealis had colonised shorelines around the Arctic via driftwood. This small asellote isopod inhabits burrows of the wood-boring isopod Limnoria borealis, which also has a circum-Arctic distribution. A similar mechanism has been inferred for the isopod Synidotea bicuspida (Svavarsson 1982), which also features a circum-Arctic distribution (Svavarsson 1979). Dispersal of this species might occur via boulders and pebbles frozen into icebergs (J. Svavarsson, personal communication).

Peracarid crustaceans are not only found in high diversity but also in very high abundances on floating items. Single species may occur in hundreds of individuals on one floating item, underlining their important role in rafting assemblages. As pointed out above, we consider that rafting dispersal may play an important role in the life history of many peracarid crustaceans.

\section{Mollusca (Polyplacophora)}

Only few polyplacophorans have been reported or inferred to be dispersed via rafting (Knox 1954, Dell 1972, Hobday 2000a). Macroalgae are considered to be the main floating items on which polyplacophorans are transported (Table 10). All species have been reported from giant kelp Macrocystis pyrifera, where they most likely occurred in the holdfast.

\section{Mollusca (Gastropoda)}

Gastropods have been reported from a wide variety of floating items, including wood (Donlan \& Nelson 2003), volcanic pumice (Richards 1958) and plastics (Barnes \& Fraser 2003) but they are most common on floating macroalgae (Hirosaki 1964, Butler et al. 1983, Edgar 1987, Hobday 2000a) (Table 10). Bingham (1992) observed substantial recruitment of a brooding gastropod, 


\section{MARTIN THIEL \& LARS GUTOW}

Table 10 Mollusca reported or inferred as rafting

\begin{tabular}{|c|c|c|c|c|}
\hline Species & Region & $\begin{array}{l}\text { Rafting } \\
\text { inference }\end{array}$ & $\begin{array}{l}\text { Floating } \\
\text { substratum }\end{array}$ & Reference \\
\hline \multicolumn{5}{|l|}{ Polyplacophora } \\
\hline $\begin{array}{l}\text { Hemiarthrum } \\
\text { setulosum }\end{array}$ & $\begin{array}{l}\text { Macquarie Island, } \\
\text { Southern Chile }\end{array}$ & $\mathrm{D}$ & M & Dell 1972, Simpson 1977 \\
\hline Onithochiton neglectus & Chatham Islands & $\mathrm{D}$ & M & Knox 1954 \\
\hline Stenoplax sp. & California & $\mathrm{R}$ & M & Hobday 2000a \\
\hline \multicolumn{5}{|c|}{ Prosobranchia, Archaeogastropoda } \\
\hline Astraea sp. & Great Barrier Reef & $\mathrm{R}$ & $\mathrm{O}$ & DeVantier 1992 \\
\hline Calliostoma annulatum & California & $\mathrm{R}$ & M & Hobday 2000a \\
\hline $\begin{array}{l}\text { Calliostoma } \\
\text { exasperatus }\end{array}$ & Azores & $\mathrm{D}$ & n.i. & Morton \& Britton 2000 \\
\hline Cantharidus japonicus & Sagami Bay, Japan & $\mathrm{R}$ & M & Hirosaki 1964 \\
\hline Clanculus plebejus & Tasmania & $\mathrm{R}$ & M & Edgar 1987 \\
\hline Collisella pelta & California & $\mathrm{R}$ & M & Hobday 2000a \\
\hline Fissurella mutabilis & St. Helena & $\mathrm{R}$ & M & Arnaud et al. 1976 \\
\hline Haliotis fulgens & California & $\mathrm{R}$ & M & Bushing 1994 \\
\hline Margarites helicinus & Iceland & $\mathrm{R}$ & M & Ingólfsson 1992 \\
\hline Margarites pupillus & Northwest coast of U.S. & $\mathrm{R}$ & M & Highsmith 1985 \\
\hline Notoacmea incessa & California & $\mathrm{R}$ & M & Hobday 2000a \\
\hline $\begin{array}{l}\text { Phasianotrochus } \\
\text { eximius }\end{array}$ & Tasmania & $\mathrm{R}$ & M & Edgar 1987 \\
\hline Tegula brunnea & California & $\mathrm{R}$ & M & Hobday 2000a \\
\hline Umbonium costatum & Sagami Bay, Japan & $\mathrm{R}$ & M & Hirosaki 1964 \\
\hline \multicolumn{5}{|c|}{ Prosobranchia, Mesogastropoda } \\
\hline Aclophoropsis festiva & Tasmania & $\mathrm{R}$ & M & Edgar 1987 \\
\hline Bittium sp. & Sargasso Sea & $\mathrm{R}$ & $\mathrm{M}$ & Coston-Clements et al. 1991 \\
\hline Cingula cingillus & Azores & $\mathrm{D}$ & n.i. & Morton \& Britton 2000 \\
\hline Cingula pulcherrina & Azores & $\mathrm{D}$ & n.i. & Morton \& Britton 2000 \\
\hline Cingula semicostata & Iceland & $\mathrm{R}$ & M & Ingólfsson 1995 \\
\hline Crepidula convexa & Florida & $\mathrm{C}$ & $\mathrm{O}$ & Collin 2001 \\
\hline Crepidula norrisiarum & California & $\mathrm{R}$ & M & Hobday $2000 a^{*}$ \\
\hline Crepidula spp. & California & $\mathrm{R}$ & M & Bushing 1994 \\
\hline Cymba gracilis & & $\mathrm{C}$ & $\mathrm{O}$ & Marche-Marchad 1968 \\
\hline Epitonium auritum & Sagami Bay, Japan & $\mathrm{R}$ & M & Hirosaki 1964 \\
\hline Fossarus sp. & Panama & $\mathrm{R}$ & $\mathrm{W}$ & Donlan \& Nelson 2003 \\
\hline Hydrobia ulvae & North Sea & $\mathrm{R}$ & M & Seidel 2002 \\
\hline Janthina globosa & Sagami Bay, Japan & $\mathrm{R}$ & M & Hirosaki 1964 \\
\hline Janthina janthina & Northwest Pacific & $\mathrm{R}$ & $\mathrm{O}$ & Tsikhon-Lukanina et al. 2001a \\
\hline Lacuna pallidula & Denmark & $\mathrm{D}$ & M & Ockelmann \& Nielsen 1981 \\
\hline Lacuna parva & Denmark & $\mathrm{D}$ & M & Ockelmann \& Nielsen 1981 \\
\hline Lacuna vincta & Iceland & $\mathrm{R}$ & M & Ingólfsson 1995 \\
\hline Lacuna sp. & Northwest coast of U.S. & $\mathrm{R}$ & M & Highsmith 1985 \\
\hline $\begin{array}{c}\text { Laevilittorina } \\
\text { antarctica }\end{array}$ & & B & $\mathrm{P}$ & Barnes \& Fraser 2003 \\
\hline $\begin{array}{l}\text { Laevilittorina } \\
\text { caliginosa }\end{array}$ & South Georgia & $\mathrm{D}$ & M & Davenport \& Stevenson 1998 \\
\hline $\begin{array}{c}\text { Laevilittorina } \\
\text { heardensis }\end{array}$ & Heard Island (Kerguelen) & $\mathrm{R}$ & M & Edgar \& Burton 2000 \\
\hline
\end{tabular}




\section{RAFTING OF BENTHIC MARINE ORGANISMS}

Table 10 (continued) Mollusca reported or inferred as rafting

\begin{tabular}{|c|c|c|c|c|}
\hline Species & Region & $\begin{array}{l}\text { Rafting } \\
\text { inference }\end{array}$ & $\begin{array}{l}\text { Floating } \\
\text { substratum }\end{array}$ & Reference \\
\hline Litiopa melanostoma & $\begin{array}{l}\text { Sargasso Sea, Red Sea, off } \\
\text { San Benedicto, Gulf } \\
\text { Stream }\end{array}$ & $\mathrm{R}$ & M, V & $\begin{array}{l}\text { Richards 1958, Markkaveeva 1965, } \\
\text { Fine 1970, Butler et al. 1983, } \\
\text { Stoner \& Greening 1984, Keller } \\
\text { 1987, Coston-Clements et al. } 1991\end{array}$ \\
\hline Littoraria luteola & Eastern Australia & $\mathrm{C}$ & $\mathrm{W}$ & Reid 1986 \\
\hline Littoraria rosewateri & East Pacific & $\mathrm{C}$ & $\mathrm{W}$ & Reid 1999 \\
\hline Littoraria scabra & Indo-Pacific & $\mathrm{C}$ & $\mathrm{W}$ & Reid 1986 \\
\hline Littoraria variegata & East Pacific & $\mathrm{C}$ & $\mathrm{W}$ & Reid 1999 \\
\hline Littorina fabalis & North Sea & $\mathrm{R}$ & M & Seidel 2002 \\
\hline Littorina obtusata & Iceland & $\mathrm{R}$ & M & Ingólfsson 1995, 1998 \\
\hline Littorina saxatilis & Iceland, North Atlantic & $\mathrm{D}, \mathrm{R}$ & M & Johannesson 1988, Ingólfsson 1995 \\
\hline Littorina sitkana & $\begin{array}{l}\text { Northwest coast of U.S., } \\
\text { British Columbia }\end{array}$ & $\mathrm{G}, \mathrm{R}$ & M & $\begin{array}{l}\text { Highsmith 1985, Kyle \& Boulding } \\
2000\end{array}$ \\
\hline Maoricolpus roseus & Tasmania & $\mathrm{R}$ & M & Edgar 1987 \\
\hline Monophorus perversus & Azores & $\mathrm{D}$ & n.i. & Morton \& Britton 2000 \\
\hline Nodilittorina paytensis & East Pacific & $\mathrm{C}$ & $\mathrm{W}$ & Reid 2002 \\
\hline Norrisia norrisi & California & $\mathrm{R}$ & M & Hobday 2000a \\
\hline Rissoa sp. & Sargasso Sea & $\mathrm{R}$ & M & Coston-Clements et al. 1991 \\
\hline Skeneopsis planorbis & Iceland, Azores & $\mathrm{D}, \mathrm{R}$ & M & $\begin{array}{l}\text { Ingólfsson 1995, 1998, Morton \& } \\
\text { Britton } 2000\end{array}$ \\
\hline Tectarius viviparus & Mariana Islands & G & n.i. & Reid \& Geller 1997 \\
\hline Thylaeodus rugulosus & Azores & $\mathrm{D}$ & n.i. & Bieler 1995 \\
\hline Vermetus triquetrus & Azores & $\mathrm{D}$ & n.i. & Bieler 1995 \\
\hline \multicolumn{5}{|c|}{ Prosobranchia, Neogastropoda } \\
\hline Adelomelon brasiliana & & $\mathrm{C}$ & $\mathrm{O}$ & Marche-Marchad 1968 \\
\hline Ammonicera rota & Azores & $\mathrm{D}$ & n.i. & Morton \& Britton 2000 \\
\hline Cominella lineolata & Eastern Australia & $\mathrm{C}$ & $\mathrm{M}$ & Hoskin 1997 \\
\hline $\begin{array}{l}\text { Concholepas } \\
\text { concholepas }\end{array}$ & Southern Chile & $\mathrm{D}$ & M & Castilla \& Guiñez 2000 \\
\hline Dentimitrella sp. & Tasmania & $\mathrm{R}$ & M & Edgar 1987 \\
\hline Ilyanassa obsoleta & Connecticut & $\mathrm{R}$ & M & Edwards \& Welsh 1982 \\
\hline Lepsiella vinosa & Tasmania & $\mathrm{R}$ & M & Edgar 1987 \\
\hline Macrozafra atkinsoni & Tasmania & $\mathrm{R}$ & M & Edgar 1987 \\
\hline Mitrella carinata & California & $\mathrm{R}$ & $\mathrm{M}$ & Hobday 2000a \\
\hline Mitrolumna olivoidea & Azores & $\mathrm{D}$ & n.i. & Morton \& Britton 2000 \\
\hline Nassarius corniculus & Azores & $\mathrm{D}$ & n.i. & Morton \& Britton 2000 \\
\hline Nassarius reticulatus & Netherlands & B & n.i. & Cadée 1999 \\
\hline Nassarius sp. & California & $\mathrm{R}$ & M & Hobday 2000a \\
\hline Nucella emarginata & California & G & n.i. & Marko 1998 \\
\hline Ocinebrina aciculata & Azores & $\mathrm{D}$ & n.i. & Morton \& Britton 2000 \\
\hline Omalogyra atomus & Azores & $\mathrm{D}$ & n.i. & Morton \& Britton 2000 \\
\hline Proximitra pica & Tasmania & $\mathrm{R}$ & M & Edgar 1987 \\
\hline Raphitoma leufroyi & Azores & $\mathrm{D}$ & n.i. & Morton \& Britton 2000 \\
\hline Thais squamosa & St. Helena & $\mathrm{R}$ & $\mathrm{M}$ & Arnaud et al. 1976 \\
\hline \multicolumn{5}{|l|}{ Opisthobranchia } \\
\hline $\begin{array}{l}\text { Acanthodoris } \\
\text { falklandica }\end{array}$ & Magellan Street & $\mathrm{R}$ & M & $\begin{array}{l}\text { Schrödl 2003; M. Schrödl, personal } \\
\text { comment }\end{array}$ \\
\hline
\end{tabular}




\section{MARTIN THIEL \& LARS GUTOW}

Table 10 (continued) Mollusca reported or inferred as rafting

\begin{tabular}{|c|c|c|c|c|}
\hline Species & Region & $\begin{array}{l}\text { Rafting } \\
\text { inference }\end{array}$ & $\begin{array}{l}\text { Floating } \\
\text { substratum }\end{array}$ & Reference \\
\hline Acanthodoris pilosa & North Sea & $\mathrm{R}$ & M & Vallentin $1895^{*}$ \\
\hline Adalaria proxima & United Kingdom & $\mathrm{G}, \mathrm{R}$ & M & Todd et al. 1998 \\
\hline Aeolidia papillosa & Iceland & $\mathrm{R}$ & M & Ingólfsson 1995 \\
\hline Aeolidiella occidentalis & $\begin{array}{l}\text { Northwest Atlantic, } \\
\text { Sargasso Sea }\end{array}$ & $\mathrm{R}$ & M & $\begin{array}{l}\text { Adams 1960, Coston-Clements } \\
\text { et al. } 1991\end{array}$ \\
\hline Aplysia dactyomela & $\begin{array}{l}\text { Northwest Atlantic, } \\
\text { Sargasso Sea }\end{array}$ & $\mathrm{R}$ & M & $\begin{array}{l}\text { Adams } 1960^{*} \text {, Coston-Clements } \\
\text { et al. } 1991^{*}\end{array}$ \\
\hline Aplysia fasciata & North Sea & $\mathrm{R}$ & M & Vallentin $1895^{*}$ \\
\hline Aplysia hirasei & Sagami Bay, Japan & $\mathrm{R}$ & M & Hirosaki 1964 \\
\hline Cavolina lonigrostris & Sargasso Sea & $\mathrm{R}$ & M & Fine 1970 \\
\hline Cavolinia sp. & Sagami Bay, Japan & $\mathrm{R}$ & M & Hirosaki 1964 \\
\hline Corambe lucea & Chile coast & $\mathrm{R}$ & M & $\begin{array}{l}\text { Schrödl 2003; M. Schrödl, personal } \\
\text { comment }\end{array}$ \\
\hline Corambe pacifica & California & $\mathrm{R}$ & M & Bushing 1994 \\
\hline Corambella depressa & $\begin{array}{l}\text { Sargasso Sea, Gulf } \\
\text { Stream }\end{array}$ & $\mathrm{R}$ & M & $\begin{array}{l}\text { Butler et al. 1983, Stoner \& } \\
\text { Greening } 1984 \text {, Coston-Clements } \\
\text { et al. } 1991\end{array}$ \\
\hline Coryphella capensis & St. Helena & $\mathrm{R}$ & M & Arnaud et al. 1976 \\
\hline Creseis virgula & Sargasso Sea & $\mathrm{R}$ & M & Fine 1970 \\
\hline Cuthona valentini & Magellan Street & $\mathrm{R}$ & M & $\begin{array}{l}\text { Schrödl 2003; M. Schrödl, personal } \\
\text { comment }\end{array}$ \\
\hline Cuthona sp. & Sargasso Sea & $\mathrm{R}$ & M & Fine 1970 \\
\hline Doridella obscura & Sargasso Sea & $\mathrm{R}$ & M & $\begin{array}{r}\text { Fine 1970, Butler et al. 1983, } \\
\text { Coston-Clements et al. } 1991\end{array}$ \\
\hline Doto uva & Chile coast & $\mathrm{R}$ & M & $\begin{array}{l}\text { Schrödl 2003; M. Schrödl, personal } \\
\text { comment }\end{array}$ \\
\hline Doto sp. & Sargasso Sea & $\mathrm{R}$ & M & Fine 1970, Keller 1987 \\
\hline Doto sp. & Mediterranean & $\mathrm{R}$ & $\mathrm{P}$ & Aliani \& Molcard 2003 \\
\hline Facelina cyanella & Chile coast & $\mathrm{R}$ & M & $\begin{array}{l}\text { Schrödl 2003; M. Schrödl, personal } \\
\text { comment }\end{array}$ \\
\hline Fiona marina & Northwest Atlantic & $\mathrm{R}$ & M & Adams 1960 \\
\hline Fiona pinnata & $\begin{array}{l}\text { Mediterranean, } \\
\text { California, Sargasso Sea, } \\
\text { Chile coast }\end{array}$ & $\mathrm{R}$ & $\mathrm{M}, \mathrm{P}, \mathrm{W}$ & $\begin{array}{l}\text { Butler et al. 1983, Keller 1987, } \\
\text { Coston-Clements et al. 1991, Il'in } \\
\text { 1992, Bushing 1994, Aliani \& } \\
\text { Molcard 2003, Schrödl 2003; M. } \\
\text { Schrödl, personal comment }\end{array}$ \\
\hline Flabellina falklandica & Magellan Street & $\mathrm{R}$ & M & $\begin{array}{l}\text { Schrödl 1999b; M. Schrödl, } \\
\text { personal comment }\end{array}$ \\
\hline Glaucus atlanticus & $\begin{array}{l}\text { Northwest Atlantic, } \\
\text { Sargasso Sea, Red Sea }\end{array}$ & $\mathrm{R}$ & M & $\begin{array}{l}\text { Adams 1960, Markkaveeva 1965, } \\
\text { Fine 1970, Coston-Clements et al. } \\
1991\end{array}$ \\
\hline Hancockia californica & California & $\mathrm{R}$ & M & $\begin{array}{l}\text { McDonald } 1983 \text { cited in Schrödl } \\
\text { 1999a }\end{array}$ \\
\hline Hancockia schoeferti & Chile coast & $\mathrm{R}$ & M & $\begin{array}{l}\text { Schrödl 2003; M. Schrödl, personal } \\
\text { comment }\end{array}$ \\
\hline Hancockia uncinata & & $\mathrm{R}$ & M & Il'in 1992 \\
\hline $\begin{array}{l}\text { Hermissenda } \\
\text { crassicornis }\end{array}$ & California & $\mathrm{R}$ & M & Bushing 1994 \\
\hline
\end{tabular}




\section{RAFTING OF BENTHIC MARINE ORGANISMS}

Table 10 (continued) Mollusca reported or inferred as rafting

\begin{tabular}{|c|c|c|c|c|}
\hline Species & Region & $\begin{array}{l}\text { Rafting } \\
\text { inference }\end{array}$ & $\begin{array}{l}\text { Floating } \\
\text { substratum }\end{array}$ & Reference \\
\hline Limenandra fusiformis & Sagami Bay, Japan & $\mathrm{R}$ & M & Hirosaki $1964 *$ \\
\hline Limenandra nodosa & & $\mathrm{R}$ & M & Il'in $1992 *$ \\
\hline Melibe leonina & California & $\mathrm{R}$ & M & Bushing 1994, Hobday 2000a \\
\hline Phyllaplysia taylori & California & $\mathrm{R}$ & $\mathrm{O}$ & Worcester 1994 \\
\hline $\begin{array}{l}\text { Pleurobranchaea } \\
\text { maculatus }\end{array}$ & Tasmania & $\mathrm{R}$ & M & Edgar 1987* \\
\hline Polycera quadrilineata & North Sea & $\mathrm{R}$ & $\mathrm{M}$ & Vallentin 1895 \\
\hline Scyllaea pelagica & $\begin{array}{l}\text { Northewst Atlantic, } \\
\text { Sargasso Sea, Sagami } \\
\text { Bay, Japan, Red Sea, } \\
\text { Azores, Gulf Stream }\end{array}$ & $\mathrm{R}$ & M & $\begin{array}{l}\text { Goodsir 1845, Hentschel 1922, } \\
\text { Adams 1960, Hirosaki 1964, } \\
\text { Markkaveeva 1965, Fine 1970, } \\
\text { Butler et al. 1983, Stoner \& } \\
\text { Greening 1984, Coston-Clements } \\
\text { et al. 1991, Morton \& Britton } 2000\end{array}$ \\
\hline Spurilla neapolitana & Sargasso Sea & $\mathrm{R}$ & M & $\begin{array}{l}\text { Adams } 1960 * \text { Fine 1970, } \\
\text { Butler et al. 1983, } \\
\text { Coston-Clements et al. } 1991\end{array}$ \\
\hline Spurilla sp. & Tasmania & $\mathrm{R}$ & M & Edgar 1987 \\
\hline Styliola subula & Sargasso Sea & $\mathrm{R}$ & M & Fine 1970 \\
\hline Stylocheilus longicauda & & $\mathrm{R}$ & M & Reznichenko 1981 \\
\hline Triopha maculata & California & $\mathrm{R}$ & M & Hobday 2000a \\
\hline Triopha spp. & California & $\mathrm{R}$ & M & Bushing 1994 \\
\hline Tylodina fungina & California & $\mathrm{R}$ & M & Bushing 1994 \\
\hline \multicolumn{5}{|l|}{ Pulmonata } \\
\hline $\begin{array}{c}\text { Amphidromus } \\
\text { porcellanus }\end{array}$ & Krakatau Islands & $\mathrm{C}$ & $\mathrm{O}$ & Thornton 1997 \\
\hline Drepanotrema lucidum & Puerto Rico & $\mathrm{R}$ & $\mathrm{O}$ & Heatwole \& Levins 1972 \\
\hline Physa marmorata & Puerto Rico & $\mathrm{R}$ & $\mathrm{O}$ & Heatwole \& Levins 1972 \\
\hline Siphonaria australis & Chatham Islands & $\mathrm{D}$ & M & Knox 1954 \\
\hline Siphonaria serrata & Southern Africa & $\mathrm{D}$ & n.i. & Chambers et al. 1998 \\
\hline \multicolumn{5}{|l|}{ Bivalvia } \\
\hline Anadara ovalis & Sargasso Sea & $\mathrm{R}$ & M & Fine 1970 \\
\hline Anomia sp. & Florida & B & $\mathrm{P}$ & Winston et al. 1997 \\
\hline Aulacomya ater & $\begin{array}{l}\text { St. Helena, Southern } \\
\text { Chile }\end{array}$ & $\mathrm{D}, \mathrm{R}$ & M & $\begin{array}{l}\text { Arnaud et al. 1976, Castilla \& } \\
\text { Guiñez } 2000\end{array}$ \\
\hline Bankia australis & Australia & B & W & Si et al. 2000 \\
\hline Cerastoderma edule & North Sea & $\mathrm{R}$ & M & Vallentin $1895^{*}$ \\
\hline Chaceia ovoidea & Baja California & $\mathrm{D}$ & $\mathrm{O}$ & Shumway $1953 *$ \\
\hline Chama sp. & Florida & B & $\mathrm{P}$ & Winston et al. 1997 \\
\hline Chlamydella incubata & Philippine Sea & $\mathrm{D}$ & n.i. & Hayami \& Kase 1996 \\
\hline Chlamydella tenuissima & Philippine Sea & $\mathrm{D}$ & n.i. & Hayami \& Kase 1996 \\
\hline Chlamys asperrimus & Tasmania & $\mathrm{R}$ & $\mathrm{M}$ & Edgar 1987 \\
\hline Chlamys tincta & St. Helena & $\mathrm{R}$ & M & Arnaud et al. 1976 \\
\hline Condylocardia sp. & Philippine Sea & $\mathrm{D}$ & n.i. & Hayami \& Kase 1996 \\
\hline Cosa waikika & Philippine Sea & $\mathrm{D}$ & n.i. & Hayami \& Kase 1996 \\
\hline Crassostrea amasa & Great Barrier Reef & $\mathrm{R}$ & $\mathrm{O}$ & DeVantier 1992 \\
\hline Crassostrea sp. & Florida & B & $\mathrm{P}$ & Winston et al. 1997 \\
\hline
\end{tabular}




\section{MARTIN THIEL \& LARS GUTOW}

Table 10 (continued) Mollusca reported or inferred as rafting

\begin{tabular}{|c|c|c|c|c|}
\hline Species & Region & $\begin{array}{l}\text { Rafting } \\
\text { inference }\end{array}$ & $\begin{array}{l}\text { Floating } \\
\text { substratum }\end{array}$ & Reference \\
\hline Cуатіuт minutum & Iceland & $\mathrm{R}$ & M & Ingólfsson 1995, 1998 \\
\hline Dacrydium zebra & Philippine Sea & $\mathrm{D}$ & n.i. & Hayami \& Kase 1996 \\
\hline Electroma georgianus & Tasmania & $\mathrm{R}$ & M & Edgar 1987 \\
\hline Gaimardia trapesina & $\begin{array}{l}\text { Southern Chile, Falkland } \\
\text { Current, Scotia Arc }\end{array}$ & $\mathrm{D}, \mathrm{R}$ & M & $\begin{array}{l}\text { Hentschel 1922, Helmuth et al. } \\
\text { 1994, Castilla \& Guiñez } 2000\end{array}$ \\
\hline Geukensia demissa & New England & $\mathrm{R}$ & $\mathrm{O}$ & Hardwick-Witman 1985 \\
\hline Hiatella arctica & St. Helena & $\mathrm{R}$ & M & Arnaud et al. 1976 \\
\hline Hiatella australis & Tasmania & $\mathrm{R}$ & M & Edgar 1987 \\
\hline Isognomon sp. & Florida & B & $\mathrm{P}$ & Winston et al. 1997 \\
\hline Kidderia bicolor & South Georgia & $\mathrm{D}$ & M & Davenport \& Stevenson 1998 \\
\hline Lasaea adansoni & Azores & $\mathrm{C}$ & $\mathrm{M}, \mathrm{W}$ & Morton \& Britton 2000 \\
\hline Lasaea spp. & Northeast Pacific & $\mathrm{D}$ & n.i. & Ó Foighil \& Eernisse 1988 \\
\hline Lasaea spp. & North Atlantic & G & n.i. & Ó Foighil \& Jozefowicz 1999 \\
\hline $\begin{array}{l}\text { Leptopecten } \\
\text { monotimeris }\end{array}$ & California & $\mathrm{R}$ & M & Hobday $2000 a$ \\
\hline Limatula pygmaea & $\begin{array}{l}\text { Falkland \& Marion } \\
\text { Islands }\end{array}$ & G & M & Page 2001, Page \& Linse 2002 \\
\hline Lopha cristagalli & New Zealand & B & $\mathrm{P}$ & Winston et al. 1997 \\
\hline Lyrodus pedicellatus & $\begin{array}{l}\text { Equatorial Current } \\
\text { (Atlantic) }\end{array}$ & $\mathrm{D}$ & $\mathrm{W}$ & $\begin{array}{l}\text { Turner } 1966 \text { cited in Scheltema } \\
\text { 1977, Scheltema } 1995\end{array}$ \\
\hline $\begin{array}{l}\text { Modiolus modiolus } \\
\text { difficilis }\end{array}$ & Japan & $\mathrm{R}$ & M & Sano et al. 2003 \\
\hline Mysella charcoti & Southern Shetland Islands & $\mathrm{R}$ & M & $\begin{array}{l}\text { Arnaud 1974, cited in Domaneschi } \\
\text { et al. } 2002\end{array}$ \\
\hline Mytilus edulis & $\begin{array}{l}\text { Irish Sea, Northwest coast } \\
\text { of U.S., Sagami Bay, } \\
\text { Japan, Iceland, North } \\
\text { Sea }\end{array}$ & $\mathrm{C}, \mathrm{R}$ & $\mathrm{M}, \mathrm{O}, \mathrm{P}$ & $\begin{array}{l}\text { Vallentin 1895, Hirosaki 1964, } \\
\text { Reineck } 1976 \text { cited in Gerlach } \\
\text { 1977, Highsmith 1985, Davenport } \\
\text { \& Rees 1993, Shaffer et al. 1995, } \\
\text { Ingólfsson 1995, } 1998\end{array}$ \\
\hline Mytilus sp. & California & $\mathrm{R}$ & M & Hobday $2000 a$ \\
\hline Nototeredo norvegica & Azores & $\mathrm{R}$ & $\mathrm{W}$ & Morton \& Britton 2000 \\
\hline Ostrea chilensis & Southern Chile & $\mathrm{D}, \mathrm{R}$ & M, V & $\begin{array}{l}\text { Ó Foighil et al. 1999, Castilla \& } \\
\text { Guiñez } 2000\end{array}$ \\
\hline Ostrea sp. & Thailand & $\mathrm{R}$ & $\mathrm{O}$ & Hamada 1964 \\
\hline Penitella penita & Baja California & $\mathrm{D}$ & $\mathrm{O}$ & Shumway $1953^{*}$ \\
\hline Philobrya sp. & Southern Ocean & $\mathrm{D}$ & M & Dell 1972 \\
\hline Pinctada albina & Great Barrier Reef & $\mathrm{R}$ & $\mathrm{O}$ & DeVantier 1992 \\
\hline Pinctada maculata & Great Barrier Reef & $\mathrm{R}$ & $\mathrm{O}$ & DeVantier 1992 \\
\hline Pinctada sp. & Florida & B & $P$ & Winston et al. 1997 \\
\hline Pteria sp. & Florida & B & $\mathrm{P}$ & Winston et al. 1997 \\
\hline $\begin{array}{l}\text { Rochefortia } \\
\text { sandwichensis }\end{array}$ & Philippine Sea & $\mathrm{D}$ & n.i. & Hayami \& Kase 1996 \\
\hline Saxicava pholadis & Baja California & $\mathrm{D}$ & $\mathrm{O}$ & Shumway 1953 \\
\hline Teredo clava & Canton (China) & B & W & Edmondson 1962 \\
\hline Teredo gregoryi & Hawaii & B & $\mathrm{W}$ & Edmondson 1962 \\
\hline Teredo megotaria & Azores & $\mathrm{R}$ & W & Morton \& Britton 2000 \\
\hline Teredo palauensis & Palau & B & W & Edmondson 1962 \\
\hline Xylophaga norvegica & Azores & $\mathrm{R}$ & W & Morton \& Britton 2000 \\
\hline
\end{tabular}




\section{RAFTING OF BENTHIC MARINE ORGANISMS}

Table 10 (continued) Mollusca reported or inferred as rafting

\begin{tabular}{|c|c|c|c|c|}
\hline Species & Region & $\begin{array}{l}\text { Rafting } \\
\text { inference }\end{array}$ & $\begin{array}{l}\text { Floating } \\
\text { substratum }\end{array}$ & Reference \\
\hline \multicolumn{5}{|l|}{ Cephalopoda } \\
\hline Argonauta sp. & Panama & $\mathrm{R}$ & W & Donlan \& Nelson 2003 \\
\hline Idiosepius pygmaeus & Sagami Bay, Japan & $\mathrm{R}$ & M & Hirosaki 1964 \\
\hline Loligo sp. & Sagami Bay, Japan & $\mathrm{R}$ & M & Hirosaki 1964 \\
\hline Octopus bimaculatus & California & $\mathrm{R}$ & M & Bushing 1994 \\
\hline Octopus bimaculoides & California & $\mathrm{R}$ & M & Hobday 2000a \\
\hline Octopus micropyrsus & California & $\mathrm{R}$ & M & Hobday 2000a \\
\hline Octopus variabilis & Sagami Bay, Japan & $\mathrm{R}$ & M & Hirosaki 1964 \\
\hline Octopus vulgaris & Sagami Bay, Japan & $\mathrm{R}$ & M & Hirosaki 1964 \\
\hline Onychia caribaea & $\begin{array}{l}\text { Northwest Atlantic, } \\
\text { Sargasso Sea }\end{array}$ & $\mathrm{R}$ & M & $\begin{array}{l}\text { Adams } 1960 \text {, Coston-Clements } \\
\text { et al. } 1991\end{array}$ \\
\hline Sepiola birostrata & Sagami Bay, Japan & $\mathrm{R}$ & M & Hirosaki 1964 \\
\hline Sepioteuthis lessoniana & Sagami Bay, Japan & $\mathrm{R}$ & M & Hirosaki 1964 \\
\hline
\end{tabular}

Notes: $\mathrm{B}=$ stranded floating item on beach; $\mathrm{C}=$ circumstantial; $\mathrm{D}=$ distributional inference; $\mathrm{G}=$ genetic inference; $\mathrm{M}$ = macroalgae; n.i. = no information; $\mathrm{O}=$ other; $\mathrm{P}=$ plastics $; \mathrm{R}=$ rafting; $\mathrm{V}=$ volcanic pumice; $\mathrm{W}=$ wood .

* References refer to this species under a different name.

Crepidula convexa, on tiles attached to mangrove roots. Possibly, recruits of these snails arrived on floating substrata (see also Collin 2001). Small snails such as the mud snail Hydrobia ulvae can also float by extending their foot, and utilising the surface tension of the sea surface they are dispersed by tidal currents over variable distances (e.g., Armonies \& Hartke 1995, Adachi \& Wada 1999), thus being independent from rafting. However, larger individuals or species depend on floating items to become dispersed via water currents.

Many prosobranch gastropods have been found on floating macroalgae. Three different gastropod species were found on floating kelp off St. Helena (Arnaud et al. 1976). In particular, small species and species with benthic egg masses are inferred to have been dispersed via rafting (Johannesson 1988, Ingólfsson 1992, 1995, 1998, Davenport \& Stevenson 1998, Hobday 2000a). Large prosobranch species may not be able to hold on to floating items. Rafting for these species may only be possible via rafting of egg masses. Leal \& Bouchet (1991) suggested rafting as a possible mechanism of dispersal for prosobranchs along the Vitória-Trinidade Seamount Chain extending about $1100 \mathrm{~km}$ eastward from the Brazilian coast. The number of directly developing species inhabiting the seamounts varied little with distance from the mainland coast, indicating that dispersal via rafting is as efficient as larval dispersal over distances of about $100-250 \mathrm{~km}$. Since only the largest species of their study, Bullata sp., was restricted to only one locality, they inferred that species with smaller eggs, juveniles and adults are probably better suited for rafting than larger ones.

Nudibranchs are among the most diverse representatives of the rafting fauna. Most species feed on hydroids and bryozoans growing on floating items (Geiselmann 1983, Il'in \& Roginskaya 1987, Schrödl 1999b). They occur primarily on macroalgae (Butler et al. 1983, Bushing 1994). The nudibranch Adalaria proxima has been observed on entangled macroalgae, and rafting may occur under certain conditions (Todd et al. 1998). Nudibranchs have also been reported from floating plastics in the Mediterranean (Aliani \& Molcard 2003). However, the two species encountered (Doto sp. and Fiona pinnata) were not very abundant and represented only a small fraction of the total community of 14 species on floating abiotic objects. Many ophistobranch species also attach their eggs to algae and seagrasses. Worcester (1994) found egg masses of Phyllaplysia taylori and other opisthobranchs on floating seagrass in Tomales Bay, California. 


\section{MARTIN THIEL \& LARS GUTOW}

A few pulmonate species have also been found on floating items (Heatwole \& Levins 1972), and based on their disjunct geographic distribution pattern others were inferred to be dispersed via rafting (Knox 1954). The species Siphonaria serrata features direct development yet occurs in widely separated regions (Chambers et al. 1998), probably having been transported via rafting.

Distributional evidence has also been used to infer rafting for mesogastropods from the family Littorinidae with direct development (Johannesson 1988, Reid 1999). Cunningham \& Collins (1998) suggested that the neogastropod Nucella lapillus, which shows little genetic divergence between northeastern and northwestern Atlantic shores, might have achieved recent genetic connection via rafting since they have non-planktotrophic larvae.

\section{Mollusca (Bivalvia)}

Most bivalve species found on floating items are small, reaching adult body sizes of a few $\mathrm{mm}$. However, large-scale range extension via rafting has also been inferred for a few intermediate-sized species such as the flat oyster Ostrea chilensis (Ó Foighil et al. 1999). Bivalves have been reported from many different substrata including macroalgae (Helmuth et al. 1994), floating corals (DeVantier 1992), Nautilus shells (Hamada 1964, Chirat 2000) and volcanic pumice (Jokiel 1984, 1989) (Table 10). On floating macroalgae in the southeast Atlantic, Arnaud et al. (1976) counted seven individuals of three different bivalve species ranging from 4-9 $\mathrm{mm}$ in shell length.

Members of the genus Lasaea have been frequently collected from floating substrata (Rosewater 1975 cited in Ó Foighil 1989). The small size of these species, their intertidal crevice-dwelling lifestyle, byssal attachment and brooding of directly developing juveniles (in many Lasaea species) are considered as morphological, behavioural and ecological pre-adaptations to rafting (Ó Foighil 1989, Ó Foighil \& Jozefowicz 1999).

While Davenport \& Rees (1993) characterised the finding of a small individual of Mytilus edulis on a piece of floating macroalgae as unexpected, Ingólfsson (1998) reported that large numbers of this bivalve recruited to floating macroalgae tethered in nearshore habitats. Small numbers of $M$. edulis have also been collected from floating seaweeds by Shaffer et al. (1995). Citing Reineck (1976), Gerlach (1977) mentioned that individuals of M. edulis have been carried out of the inner German Wadden Sea (North Sea) by ice. The animals become frozen to the ice when drift ice is stranded on the sediment during low tide. When the water rises during flood tide the ice blocks refloat with the attached sediment and organisms. The same mechanism has been described by Hardwick-Witman (1985) for the ribbed mussel Geukensia demissa from salt marshes in New England. Ice rafting of $G$. demissa from the high intertidal salt marsh habitat to the low intertidal caused enormous mortality among the mussels. Dislodgement of mussels was enhanced by the overgrowth of the animals with plants of the brown alga Fucus vesiculosus.

Hamada (1964) mentioned that there were between one and two individuals of an oyster (Ostrea sp.) per stranded shell of Nautilus pompilius found on beaches in Thailand. Winston et al. (1997) also reported rafting oysters on plastics and DeVantier (1992) found the oyster Crassostrea amasa on floating corals.

Postlarval bivalves of the species Macoma baltica are transported in the water column by thread-drifting (Beukema \& Vlas 1989). This mechanism has been observed to play an important role in the life history of many marine bivalve species (Martel \& Chia 1991). Frequent excursions in the water column by the use of widely extending mucus threads can be expected to increase the possibility of an encounter of the animals with floating objects, which might enhance the chances of long-distance epipelagic transport (Martel \& Chia 1991). The frequent occurrence of threaddrifting (and subsequent rafting) in brooding bivalves (e.g., Musculus sp., Lasaea sp., Transenella tantilla) or in bivalves that produce benthic egg capsules or crawl-away juveniles (e.g., Barleeia 


\section{RAFTING OF BENTHIC MARINE ORGANISMS}

spp.) has been held responsible for the often wide geographic distribution of many bivalve species that lack planktonic larvae (Martel \& Chia 1991).

Geographical evidence has been used to infer rafting for many small and brooding bivalves (Buroker et al. 1983, Ó Foighil 1989, Helmuth et al. 1994, Ó Foighil \& Jozefowicz 1999, Castilla \& Guiñez 2000). Vermeij et al. (1990) attributed the spread of several brooding mollusc species (bivalves as well as gastropods) along the chain of the Aleutian Islands to rafting. However, no rafting specimens were reported because sampling was limited to the benthos.

Wood-boring bivalves (Teredinidae) are among the most common rafting organisms on/in wood. Edmondson $(1946,1962)$ suggested that the extensive distribution of the species Teredo gregoryi in the North Pacific and T. clava in the Indian Ocean is the consequence of rafting dispersal in wood.

\section{Mollusca (Cephalopoda)}

Dooley (1972) listed cephalopods as one of the major groups of the Sargassum community. In Tasmanian waters, however, octopuses quickly vanished from Macrocystis pyrifera holdfasts after the plants were detached from their substratum (Edgar 1987).

\section{Echinodermata}

Echinoderms were primarily collected from floating macroalgae (Mortensen 1933, Hirosaki 1964, Edgar 1987, Bushing 1994, Hobday 2000a) (Table 11). The brooding seastar Leptasterias hexactis has been reported from the brown alga Nereocystis luetkeana, and Henricia leviuscula from another floating alga (Bushing 1994). Arnaud et al. (1976) collected brittle stars as well as sea urchins from the haptera of floating kelp at St. Helena. Holdfasts of Macrocystis pyrifera in Tasmanian waters, too, are inhabited by echinoderms (Edgar 1987). The brittle star Amphipholis squamata is a common species on floating macroalgae (Highsmith 1985, Edgar 1987, Bushing 1994, O'Hara 1998). Rafting dispersal might explain the cosmopolitan distribution of this species (Sponer \& Roy 2002). Mortensen (1933) concluded from the absence of a pelagic larval stage during development of Asterina exigua (= Patiriella exigua) that this species reached St. Helena from South Africa via rafting most likely with floating Ecklonia maxima (see also Waters \& Roy 2004).

Considering geographic distribution, depth range, habitat and life history characteristics of 42 echinoderm species from Macquarie Island in the Southern Ocean, O'Hara (1998) inferred for about $20 \%$ of the species that rafting on floating macroalgae from the Kerguelen Islands is the most probable mechanism of transport to that remote island. Due to the lack of morphological differentiation between echinoderms from the Macquarie and Kerguelen islands, the author assumed that there is still ongoing gene flow between these locations. Suggested rafters were exclusively holothurians and asteroids, which are probably able to secure themselves within the holdfasts of floating macroalgae by using comparatively large suctorial tube feet. Due to the minor efficiency of these specialised structures in ophiuroids and echinoids, rafting was considered an unlikely dispersal mechanism for these taxa even though they were quite abundant in kelp beds around the island. This assumption was supported by Vásquez (1993) who experimentally revealed that asteroids survived better on detached macroalgae than ophiuroids and echinoids did. Echinoderms are also found on anthropogenic debris. The sea urchin Arbacia lixula is a member of a diverse fauna on floating debris in the Mediterranean (Aliani \& Molcard 2003).

Juveniles suspended in the water column have also been mentioned for the directly developing seastar Leptasterias hexactis by Martel \& Chia (1991) who found some specimens in intertidal offbottom collectors. In contrast to young bivalves, floating of juvenile echinoderms is not facilitated by mucus threads. Nevertheless, suspension in the pelagial enhances the probability of encountering other floating objects and subsequent rafting. 


\section{MARTIN THIEL \& LARS GUTOW}

Table 11 Echinodermata reported or inferred as rafting

\begin{tabular}{|c|c|c|c|c|}
\hline Species & Region & $\begin{array}{l}\text { Rafting } \\
\text { inference }\end{array}$ & $\begin{array}{l}\text { Floating } \\
\text { substratum }\end{array}$ & Reference \\
\hline \multicolumn{5}{|l|}{ Crinoidea } \\
\hline Comanthus sp. & Sagami Bay, Japan & $\mathrm{R}$ & M & Hirosaki 1964 \\
\hline \multicolumn{5}{|l|}{ Asteroidea } \\
\hline Anasterias directa & Macquarie Island & $\mathrm{D}$ & n.i. & O'Hara 1998 \\
\hline Anasterias mawsoni & $\begin{array}{l}\text { Macquarie Island, Heard } \\
\text { Island (Kerguelen) }\end{array}$ & $\mathrm{D}$ & M & $\begin{array}{l}\text { O'Hara 1998, Edgar \& Burton } \\
2000\end{array}$ \\
\hline Anasterias sp. & Southern Ocean & $\mathrm{R}$ & M & $\begin{array}{l}\text { Mortensen 1925*, cited in Fell } \\
1967\end{array}$ \\
\hline Asterina hamiltoni & Macquarie Island & $\mathrm{D}$ & n.i. & O'Hara 1998 \\
\hline Asterina miniata & California & $\mathrm{R}$ & M & Hobday 2000a* \\
\hline Coscinasterias acutispina & Sagami Bay, Japan & $\mathrm{R}$ & M & Hirosaki 1964 \\
\hline Coscinasterias calamaria & Tasmania & $\mathrm{R}$ & M & Edgar 1987 \\
\hline Coscinasterias muricata & Australia & G & n.i. & Waters \& Roy 2003 \\
\hline Henricia leviuscula & California & $\mathrm{R}$ & $\mathrm{M}$ & Bushing 1994 \\
\hline Leptasterias hexactis & $\begin{array}{l}\text { California, Northwest } \\
\text { coast of U.S. }\end{array}$ & $\mathrm{R}$ & M & $\begin{array}{l}\text { Highsmith 1985, Bushing } \\
1994\end{array}$ \\
\hline Linckia columbiae & California & $\mathrm{R}$ & M & Bushing 1994 \\
\hline Patiriella exigua & $\begin{array}{l}\text { New South Wales, } \\
\text { St. Helena, Southern } \\
\text { Ocean }\end{array}$ & $\mathrm{D}, \mathrm{G}, \mathrm{R}$ & M & $\begin{array}{l}\text { Mortensen 1933*, Fell 1967, } \\
\text { Byrne 1995, Hart et al. 1997, } \\
\text { Waters \& Roy } 2004\end{array}$ \\
\hline Pisaster sp. & California & $\mathrm{R}$ & M & Hobday 2000a \\
\hline Porania antarctica & Macquarie Island & $\mathrm{D}$ & n.i. & O'Hara 1998 \\
\hline Pteraster affinis affinis & Macquarie Island & $\mathrm{D}$ & $\mathrm{M}$ & O'Hara 1998 \\
\hline \multicolumn{5}{|l|}{ Echinoidea } \\
\hline Arbacia lixula & Mediterranean & $\mathrm{R}$ & $\mathrm{P}$ & Aliani \& Molcard 2003 \\
\hline Echinocardium cordatum & Tasmania & $\mathrm{R}$ & M & Edgar 1987 \\
\hline Heliocidaris sp. & $\begin{array}{l}\text { Aotea seamount, } 1200 \\
\text { miles east of Australia }\end{array}$ & $\mathrm{D}$ & M & Fell 1962, cited in Fell 1967 \\
\hline Lytechinus anamesus & California & $\mathrm{R}$ & M & Hobday 2000a \\
\hline Parechinus angulosus & St. Helena & $\mathrm{R}$ & M & Arnaud et al. 1976 \\
\hline Pseudocentrotus depressus & Sagami Bay, Japan & $\mathrm{R}$ & M & Hirosaki 1964 \\
\hline Strongylocentrotus purpuratus & California & $\mathrm{R}$ & M & Hobday 2000a \\
\hline \multicolumn{5}{|l|}{ Holothuroidea } \\
\hline Eupenctata quinquesemita & California & $\mathrm{R}$ & M & Bushing 1994, Hobday 2000a \\
\hline Leptosynapta clarki & $\begin{array}{l}\text { Northwest coast of U.S., } \\
\text { Vancouver Island }\end{array}$ & $\mathrm{R}$ & M & Highsmith 1985, Sewell 1994 \\
\hline Pentacta australis & Tasmania & $\mathrm{R}$ & $\mathrm{M}$ & Edgar 1987 \\
\hline Pseudocnus laevigatus & Macquarie Island & $\mathrm{D}$ & n.i. & O'Hara 1998 \\
\hline Pseudopsolus macquariensis & Macquarie Island & $\mathrm{D}$ & n.i. & O'Hara 1998 \\
\hline Trachythyone macphersonae & Macquarie Island & $\mathrm{D}$ & n.i. & O'Hara 1998 \\
\hline \multicolumn{5}{|l|}{ Ophiuroidea } \\
\hline Amphiodia occidentalis & California & $\mathrm{R}$ & M & Bushing 1994 \\
\hline Amphipholis squamata & $\begin{array}{l}\text { California, Tasmania, } \\
\text { Northwest coast of U.S., } \\
\text { St. Helena }\end{array}$ & $\mathrm{R}$ & M & $\begin{array}{c}\text { Mortensen 1933, Highsmith } \\
\text { 1985, Edgar 1987, Bushing } \\
\text { 1994, Sponer \& Roy } 2002\end{array}$ \\
\hline Amphiura capensis & Tristan da Cunha & $\mathrm{R}$ & M & Mortensen 1941 \\
\hline
\end{tabular}




\section{RAFTING OF BENTHIC MARINE ORGANISMS}

Table 11 (continued) Echinodermata reported or inferred as rafting

\begin{tabular}{lllcl}
\hline Species & Region & $\begin{array}{l}\text { Rafting } \\
\text { inference }\end{array}$ & $\begin{array}{l}\text { Floating } \\
\text { substratum }\end{array}$ & Reference \\
\hline Amphiura magellanica & Southern Ocean & $\mathrm{R}$ & $\mathrm{M}$ & $\begin{array}{c}\text { Fell 1953, cited in Hendler } \\
1991\end{array}$ \\
Amphiura sp. & Tasmania & $\mathrm{R}$ & $\mathrm{M}$ & Edgar 1987 \\
Ophiactis carnea & St. Helena & $\mathrm{R}$ & $\mathrm{M}$ & Arnaud et al. 1976 \\
Ophiactis simplex & California & $\mathrm{R}$ & $\mathrm{M}$ & Hobday 2000a \\
Ophioplocus esmarki & California & $\mathrm{R}$ & $\mathrm{M}$ & Bushing 1994 \\
Ophiothrix caespitosa & Tasmania & $\mathrm{R}$ & $\mathrm{M}$ & Edgar 1987 \\
Ophiothrix spiculata & California & $\mathrm{R}$ & $\mathrm{M}$ & Hobday 2000a \\
Ophiothrix triglochis & St. Helena & $\mathrm{R}$ & $\mathrm{M}$ & Arnaud et al. 1976 \\
\hline
\end{tabular}

Notes: $\mathrm{D}=$ distributional inference; $\mathrm{G}=$ genetic inference; $\mathrm{M}=$ macroalgae; $\mathrm{n} . \mathrm{i}$. = no information; $\mathrm{R}=$ rafting.

* References refer to this species under a different name.

Ectoprocta $(=$ Bryozoa $)$

Some of the most ubiquitous organisms on floating plastic items are bryozoans (Winston 1982, Stevens et al. 1996, Winston et al. 1997, Barnes \& Sanderson 2000). Barnes (2002) ranked the bryozoa as the most abundant faunal group on marine debris. Bryozoans have been found on a variety of different floating substrata including macroalgae (Arnaud et al. 1976, Niermann 1986, Bushing 1994), seagrass blades (Keough \& Chernoff 1987, Worcester 1994), seeds (Mason 1961), Nautilus shells (Hamada 1964, Chirat 2000), floating corals (DeVantier 1992), plastic items (Gregory 1978, Winston 1982, Stevens et al. 1996, Winston et al. 1997, Barnes \& Sanderson 2000, Donlan \& Nelson 2003) and tar balls (Shaw \& Mapes 1979) (Table 12). Gregory (1978) suggested that the cosmopolitan bryozoan Membranipora tuberculata, which is also very common on floating Sargassum in the Sargasso Sea (Smith et al. 1973, Ryland 1974), may have crossed the Tasman Sea from Australia to New Zealand via rafting on plastic pellets. Winston (1982) found Electra tenella to be the dominating and often only bryozoan species on plastic debris cast up on beaches along the Atlantic coast of Florida. In benthic habitats, however, this species occurs only sparsely in that region. Winston (1982) predicted the niche of this species to expand spatially because of the capability of the animals to utilise an increasing amount of anthropogenic debris floating in the world's oceans as a habitat. On natural substrata such as floating Sargassum, Electra tenella is lacking, which could either be due to settlement preferences of this species or due to its competitive inferiority to the dominating species on floating Sargassum in the North Atlantic, Membranipora tuberculata. Similar as M. tuberculata, the erect bryozoan Bugula neritina can be found on a wide variety of different substrata including seagrass blades (Keough \& Chernoff 1987) and plastic surfaces (Thiel et al. 2003).

In the western Mediterranean, Aliani \& Molcard (2003) found four species of Bryozoa on floating debris. While colonisation frequency decreased towards higher latitudes (Winston et al. 1997, Barnes \& Sanderson 2000), bryozoans were still the most common sessile organisms reported from floating plastics in the Southern Ocean (Barnes \& Fraser 2003). Based on geographic evidence and a meta-analysis of British bryozoans, Watts et al. (1998) suggested that rafting played an important role in evolution and biogeography of cheilostome bryozoans (albeit in present-day oceans transport via ships may overshadow rafting dispersal). 


\section{MARTIN THIEL \& LARS GUTOW}

Table 12 Ectoprocta (Bryozoa) and Chaetognatha reported or inferred as rafting

\begin{tabular}{|c|c|c|c|c|}
\hline Species & Region & $\begin{array}{l}\text { Rafting } \\
\text { inference }\end{array}$ & $\begin{array}{l}\text { Floating } \\
\text { substratum }\end{array}$ & Reference \\
\hline \multicolumn{5}{|l|}{ Ectoprocta (Bryozoa) } \\
\hline Aetea anguina & Sargasso Sea & $\mathrm{R}$ & M & $\begin{array}{l}\text { Butler et al. 1983, Coston- } \\
\text { Clements et al. } 1991\end{array}$ \\
\hline Aetea sica & Brazil Current & $\mathrm{R}$ & M & Hentschel 1922* \\
\hline Aimulosia antarctica & Antarctic peninsula & B & $\mathrm{P}$ & Barnes \& Fraser 2003 \\
\hline Aimulosia marsupium & New Zealand & B & $\mathrm{P}$ & Stevens et al. 1996 \\
\hline Amphiblestrum contentum & New Zealand & B & $\mathrm{P}$ & Stevens et al. 1996 \\
\hline Arachnopusia inchoata & Antarctic peninsula & B & $\mathrm{P}$ & Barnes \& Fraser 2003 \\
\hline Arachnopusia unicornis & New Zealand & B & $\mathrm{P}$ & Stevens et al. 1996 \\
\hline Arthropoma cecilii & Sagami Bay, Japan & $\mathrm{R}$ & M & Hirosaki 1964 \\
\hline Beania discodermiae & Sagami Bay, Japan & $\mathrm{R}$ & M & Hirosaki 1964 \\
\hline $\begin{array}{l}\text { Beania inermis } \\
\text { cryptophragma }\end{array}$ & New Zealand & B & $\mathrm{P}$ & Stevens et al. 1996 \\
\hline Beania plurispinosa & New Zealand & B & $\mathrm{P}$ & Stevens et al. 1996 \\
\hline Bicellariella ciliata & & $\mathrm{R}$ & M & Vallentin $1895^{*}$ \\
\hline Bowerbankia gracilis & Mediterranean, North Pacific & $\mathrm{R}$ & $\mathrm{O}, \mathrm{P}$ & $\begin{array}{l}\text { Sinitsyn \& Reznichenko 1981, } \\
\text { Aliani \& Molcard } 2003\end{array}$ \\
\hline Bowerbankia sp. & Sargasso Sea & $\mathrm{R}$ & M & Hentschel 1922 \\
\hline Bugula flabellata & New Zealand & B & $\mathrm{P}$ & Stevens et al. 1996 \\
\hline Bugula minima & New Zealand & B & $\mathrm{P}$ & Winston et al. 1997 \\
\hline Bugula neritina & $\begin{array}{l}\text { Sagami Bay, Japan, Gulf of } \\
\text { Mexico }\end{array}$ & $\mathrm{R}$ & M & $\begin{array}{l}\text { Hirosaki 1964, Keough \& } \\
\text { Chernoff 1987, Thiel et al. } \\
2003\end{array}$ \\
\hline Caberea lata & Sagami Bay, Japan & $\mathrm{R}$ & M & Hirosaki 1964 \\
\hline Caberea rostrata & New Zealand & B & $\mathrm{P}$ & Stevens et al. 1996 \\
\hline Caberea zelandica & New Zealand & B & $\mathrm{P}$ & Stevens et al. 1996 \\
\hline Calyptotheca immersa & New Zealand & B & $\mathrm{P}$ & Stevens et al. 1996 \\
\hline Callopora lineata & Mediterranean & $\mathrm{R}$ & $\mathrm{P}$ & Aliani \& Molcard 2003 \\
\hline Calloporina angustipora & New Zealand & B & $\mathrm{P}$ & Stevens et al. 1996 \\
\hline Celleporaria agglutinans & New Zealand & B & $\mathrm{P}$ & Stevens et al. 1996 \\
\hline Celleporella tongima & New Zealand & B & $\mathrm{P}$ & Stevens et al. 1996 \\
\hline Celleporina costazii & Sagami Bay, Japan & $\mathrm{R}$ & M & Hirosaki 1964 \\
\hline $\begin{array}{l}\text { Celleporina } \\
\text { hemiperistomata }\end{array}$ & New Zealand & B & $\mathrm{P}$ & $\begin{array}{l}\text { Stevens et al. 1996, Winston } \\
\text { et al. } 1997\end{array}$ \\
\hline Chaperia acanthina & New Zealand & B & $\mathrm{P}$ & Stevens et al. 1996 \\
\hline Chaperiopsis sp. & New Zealand & B & $\mathrm{P}$ & Stevens et al. 1996 \\
\hline Chiastosella sp. & New Zealand & B & $\mathrm{P}$ & Stevens et al. 1996 \\
\hline Crepidacantha crinispina & New Zealand & B & $\mathrm{P}$ & Stevens et al. 1996 \\
\hline Crisia eburneo-denticulata & Sagami Bay, Japan & $\mathrm{R}$ & M & Hirosaki 1964 \\
\hline Cryptosula pallasiana & New Zealand & B & $\mathrm{P}$ & $\begin{array}{l}\text { Stevens et al. 1996, Winston } \\
\text { et al. } 1997\end{array}$ \\
\hline Disporella sp. & New Zealand & B & $\mathrm{P}$ & Stevens et al. 1996 \\
\hline Electra angulata & $\begin{array}{l}\text { Sagami Bay, Japan, } \\
\text { Indo-Pacific, New Zealand }\end{array}$ & $\mathrm{B}, \mathrm{R}$ & $\mathrm{M}, \mathrm{O}, \mathrm{P}, \mathrm{W}$ & $\begin{array}{l}\text { Hirosaki 1964, Winston 1982, } \\
\text { Winston et al. } 1997\end{array}$ \\
\hline Electra bellula & Brazil Current & $\mathrm{R}$ & M & Hentschel 1922* \\
\hline Electra pilosa & United Kingdom & $\mathrm{R}$ & M & Vallentin 1895*, Todd et al. 1998 \\
\hline Electra posidoniae & Mediterranean & $\mathrm{R}$ & $\mathrm{P}$ & Aliani \& Molcard 2003 \\
\hline
\end{tabular}




\section{RAFTING OF BENTHIC MARINE ORGANISMS}

Table 12 (continued) Ectoprocta (Bryozoa) and Chaetognatha reported or inferred as rafting

\begin{tabular}{|c|c|c|c|c|}
\hline Species & Region & $\begin{array}{l}\text { Rafting } \\
\text { inference }\end{array}$ & $\begin{array}{l}\text { Floating } \\
\text { substratum }\end{array}$ & Reference \\
\hline Electra tenella & $\begin{array}{l}\text { Panama, North Pacific, } \\
\text { Northwest Pacific, Florida, } \\
\text { New Zealand }\end{array}$ & $\mathrm{B}, \mathrm{R}$ & $\mathrm{O}, \mathrm{P}, \mathrm{W}$ & $\begin{array}{l}\text { Sinitsyn \& Reznichenko } 1981 \text {, } \\
\text { Winston 1982, Stevens et al. } \\
\text { 1996, Winston et al. 1997, } \\
\text { Tsikhon-Lukanina et al. 2001a, } \\
\text { Donlan \& Nelson 2003* }\end{array}$ \\
\hline Ellisina antarctica & Antarctic peninsula & $\mathrm{B}$ & $\mathrm{P}$ & Barnes \& Fraser 2003 \\
\hline Escharoides angela & New Zealand & B & $\mathrm{P}$ & Stevens et al. 1996 \\
\hline Escharoides excavata & New Zealand & $\mathrm{B}$ & $\mathrm{P}$ & Stevens et al. 1996 \\
\hline Eurystomella foraminigera & New Zealand & B & $\mathrm{P}$ & Stevens et al. 1996 \\
\hline Exochella armata & New Zealand & B & $\mathrm{P}$ & Stevens et al. 1996 \\
\hline Exochella tricuspis & New Zealand & B & $\mathrm{P}$ & Stevens et al. 1996 \\
\hline Favosipora $\mathrm{sp}$ & New Zealand & B & $\mathrm{P}$ & Stevens et al. 1996 \\
\hline Fenestrulina cf. disjuncta & New Zealand & B & $\mathrm{P}$ & Stevens et al. 1996 \\
\hline Fenestrulina rugula & Antarctic peninsula & B & $\mathrm{P}$ & Barnes \& Fraser 2003 \\
\hline Flustra foliacea & & $\mathrm{R}$ & M & Vallentin 1895 \\
\hline Flustra tuberculata & Northwest Atlantic & $\mathrm{R}$ & M & Adams 1960 \\
\hline Flustrellida hispida & Equatorial Atlantic & $\mathrm{R}$ & M & John 1974 \\
\hline Galeopsis polyporus & New Zealand & B & $\mathrm{P}$ & Stevens et al. 1996 \\
\hline Galeopsis porcellanicus & New Zealand & B & $\mathrm{P}$ & Stevens et al. 1996 \\
\hline Hastingsia sp. & New Zealand & B & $\mathrm{P}$ & Stevens et al. 1996 \\
\hline Hippoflustra serrata & Sagami Bay, Japan & $\mathrm{R}$ & M & Hirosaki 1964* \\
\hline Inversiula fertilis & New Zealand & $\mathrm{B}$ & $\mathrm{P}$ & Stevens et al. 1996 \\
\hline Jellyella eburnea & New Zealand & B & $\mathrm{P}$ & Stevens et al. $1996^{*}$ \\
\hline Jellyella tuberculata & Northwest Atlantic & $\mathrm{R}$ & M & Adams $1960 *$ \\
\hline Lichenopora radiata & Sagami Bay, Japan & $\mathrm{R}$ & M & Hirosaki 1964 \\
\hline Macropora grandis & New Zealand & B & $\mathrm{P}$ & Stevens et al. 1996 \\
\hline Membranipora reticulum & Brazil Current & $\mathrm{R}$ & M & Hentschel 1922 \\
\hline Membranipora arborescens & Florida & B & $\mathrm{P}$ & Winston et al. 1997 \\
\hline Membranipora hyadesi & Scotia Arc & $\mathrm{R}$ & M & Helmuth et al. 1994 \\
\hline $\begin{array}{l}\text { Membranipora } \\
\text { membranacea }\end{array}$ & Mediterranean & $\mathrm{R}$ & $\mathrm{P}$ & Aliani \& Molcard 2003 \\
\hline Membranipora savartii & Florida & $\mathrm{B}$ & $\mathrm{P}$ & Winston et al. 1997 \\
\hline Membranipora tuberculata & $\begin{array}{l}\text { Northwest Atlantic, Sargasso } \\
\text { Sea, Panama, New Zealand, } \\
\text { North Atlantic, Florida }\end{array}$ & $\mathrm{B}, \mathrm{R}$ & $\mathrm{M}, \mathrm{P}, \mathrm{W}$ & $\begin{array}{l}\text { Hentschel } 1922^{*} \text {, Adams } 1960 \text {, } \\
\text { Smith et al. 1973, Ryland } \\
\text { 1974, Scheltema 1977, } \\
\text { Gregory 1978, Butler et al. } \\
\text { 1983, Niermann 1986, } \\
\text { Coston-Clements et al. 1991, } \\
\text { Stevens et al. 1996, Winston } \\
\text { et al. 1997, Donlan \& Nelson } \\
2003\end{array}$ \\
\hline Membranipora sp. & Panama & $\mathrm{R}$ & $\mathrm{W}$ & Donlan \& Nelson 2003 \\
\hline Membranipora sp. & Gulf of Mexico & $\mathrm{R}$ & M & Johnson \& Richardson 1977 \\
\hline Membranipora sp. & Sargasso Sea & $\mathrm{R}$ & M & Keller 1987 \\
\hline Membranipora spp. & California & $\mathrm{R}$ & $\mathrm{M}$ & Bushing 1994 \\
\hline Micropora brevissima & Antarctic peninsula & B & $\mathrm{P}$ & Barnes \& Fraser 2003 \\
\hline Micropora mortensi & New Zealand & B & $\mathrm{P}$ & Stevens et al. 1996 \\
\hline
\end{tabular}




\section{MARTIN THIEL \& LARS GUTOW}

Table 12 (continued) Ectoprocta (Bryozoa) and Chaetognatha reported or inferred as rafting

\begin{tabular}{|c|c|c|c|c|}
\hline Species & Region & $\begin{array}{l}\text { Rafting } \\
\text { inference }\end{array}$ & $\begin{array}{l}\text { Floating } \\
\text { substratum }\end{array}$ & Reference \\
\hline Microporella agonistes & New Zealand & B & $\mathrm{P}$ & Stevens et al. 1996 \\
\hline Microporella ciliata & Sagami Bay, Japan & $\mathrm{R}$ & M & Hirosaki 1964 \\
\hline Microporella speculum & New Zealand & B & $\mathrm{P}$ & Stevens et al. 1996 \\
\hline Mimosella gracilis & & $\mathrm{R}$ & M & Vallentin 1895 \\
\hline Opaeophora lepida & New Zealand & B & $\mathrm{P}$ & Stevens et al. 1996 \\
\hline Parasmittina sp. & New Zealand & B & $\mathrm{P}$ & Stevens et al. 1996 \\
\hline Parasmittina trispinosa & Sagami Bay, Japan & $\mathrm{R}$ & M & Hirosaki 1964 \\
\hline Platonea sp. & New Zealand & B & $\mathrm{P}$ & Stevens et al. 1996 \\
\hline Rhynchozoon larreyi & New Zealand & B & $\mathrm{P}$ & Stevens et al. 1996 \\
\hline Schizoporella nivea & St. Helena & $\mathrm{R}$ & M & Arnaud et al. 1976 \\
\hline Schizoporella unicornis & Sagami Bay, Japan & $\mathrm{R}$ & M & Hirosaki 1964 \\
\hline Schizosmittina sp. & New Zealand & B & $\mathrm{P}$ & Stevens et al. 1996 \\
\hline Scrupocellaria scrupea & Brazil Current & $\mathrm{R}$ & M & Hentschel 1922 \\
\hline Smittina torques & New Zealand & B & $\mathrm{P}$ & Stevens et al. 1996 \\
\hline Smittoidea maunganuiensis & New Zealand & B & $\mathrm{P}$ & Stevens et al. 1996 \\
\hline Smittoidea sp. & New Zealand & B & $\mathrm{P}$ & Stevens et al. 1996 \\
\hline Steginoporella magnifica & New Zealand & B & $\mathrm{P}$ & Stevens et al. 1996 \\
\hline Thalamoporella evelinae & New Zealand & B & $\mathrm{P}$ & Winston et al. 1997 \\
\hline Thalamoporella falcifer & Sargasso Sea & $\mathrm{R}$ & M & $\begin{array}{l}\text { Butler et al. 1983, } \\
\text { Coston-Clements et al. } 1991\end{array}$ \\
\hline Tricellaria occidentalis & Sagami Bay, Japan & $\mathrm{R}$ & M & Hirosaki 1964 \\
\hline Tubulipora pulchra & Sagami Bay, Japan & $\mathrm{R}$ & M & Hirosaki 1964 \\
\hline Tubulipora sp. & New Zealand & B & $\mathrm{P}$ & Stevens et al. 1996 \\
\hline Walkeria uva & & $\mathrm{R}$ & M & Vallentin 1895 \\
\hline Watersipora subtorquata & New Zealand & $\mathrm{B}$ & $\mathrm{P}$ & $\begin{array}{l}\text { Stevens et al. 1996, Winston } \\
\text { et al. } 1997\end{array}$ \\
\hline \multicolumn{5}{|l|}{ Chaetognatha } \\
\hline Sagitta elegans & Irish Sea & $\mathrm{R}$ & M & Davenport \& Rees 1993 \\
\hline Sagitta hispida & Sargasso Sea & $\mathrm{R}$ & M & Fine 1970 \\
\hline
\end{tabular}

Notes: $\mathrm{B}=$ stranded floating item on beach; $\mathrm{M}=$ macroalgae; $\mathrm{O}=$ other; $\mathrm{P}=$ plastics; $\mathrm{R}=$ rafting; $\mathrm{W}=$ wood.

* References refer to this species under a different name.

\section{Chordata (Tunicata)}

Several compound ascidians have been found rafting on floating seagrass blades (Worcester 1994) and on macroalgae (Bushing 1994, Dooley 1972) (Table 13). In a shallow bay in California, Worcester (1994) found that rafting of colonies of Botrylloides sp. on floating blades of Zostera marina is a common phenomenon. About two-thirds of all colonies rafted during a 3-month period of investigation. Rafting is usually initiated by the breakage of senescent seagrass blades. Since blades are generally less persistent than Botrylloides colonies, the latter have a high probability to experience rafting during their lifetime. Colonies rafting on eelgrass travelled over 200 times farther than swimming larvae that swim only for a short period of a few minutes up to an hour (Worcester 1994). The author attributed the efficiency of rafting compared with larval dispersal to the fact that rafting occurs mainly during flooding tide whereas larvae are preferably released during slack tide when water movements are low. Rafting colonies had recruitment success comparable to swimming larvae by either moving or growing asexually from floating blades onto attached plants after getting 


\section{RAFTING OF BENTHIC MARINE ORGANISMS}

Table 13 Tunicata reported or inferred as rafting

\begin{tabular}{|c|c|c|c|c|}
\hline Species & Region & $\begin{array}{l}\text { Rafting } \\
\text { inference }\end{array}$ & $\begin{array}{l}\text { Floating } \\
\text { substratum }\end{array}$ & Reference \\
\hline \multicolumn{5}{|l|}{ Ascidiacea } \\
\hline Aplidium californicum & California & $\mathrm{R}$ & M & Bushing 1994 \\
\hline Boltenia villosa & California & $\mathrm{R}$ & M & Bushing 1994 \\
\hline Botrylloides violaceus & Sagami Bay, Japan & $\mathrm{R}$ & M & Hirosaki 1964 \\
\hline Botrylloides sp. & California & $\mathrm{R}$ & $\mathrm{O}$ & Worcester 1994 \\
\hline Botryllus schlosseri & California, North Sea & $\mathrm{R}$ & $\mathrm{O}$ & Worcester 1994, personal observation \\
\hline Didemnum carnulatum & California & $\mathrm{R}$ & $\mathrm{O}$ & Worcester 1994 \\
\hline Diplosoma listerianum & Sargasso Sea & $\mathrm{R}$ & M & $\begin{array}{l}\text { Hentschel } 1922 * \text {, Timmermann } \\
\text { 1932*, Butler et al. } 1983 * \\
\text { Coston-Clements et al. } 1991 *\end{array}$ \\
\hline Diplosoma sp. & Northwest Atlantic & $\mathrm{R}$ & M & Adams 1960 \\
\hline Ecteinascidia turbinata & Caribbean & $\mathrm{C}$ & $\mathrm{W}$ & Kathiresan \& Bingham 2001 \\
\hline Pyura chilensis & Southern Chile & $\mathrm{R}$ & $\mathrm{O}$ & Thiel et al. 2003 \\
\hline Pyura stolonifera & Southern Chile & $\mathrm{D}$ & M & Castilla \& Guiñez 2000 \\
\hline
\end{tabular}

Notes: $\mathrm{C}=$ circumstantial; $\mathrm{D}=$ distributional inference; $\mathrm{M}=$ macroalgae; $\mathrm{O}=$ other; $\mathrm{R}=$ rafting; $\mathrm{W}=$ wood.

* References refer to this species under a different name.

into contact with them. Large colonies were generally more likely to remain at the site of settlement than small or medium-sized colonies because large colonies frequently reattach to the surrounding substratum while smaller colonies rarely reattach. Attachment of colonies was assisted by strings of diatoms or by mucus tubes built by polychaetes.

Solitary ascidians have been reported from macroalgae (Bushing 1994) but also from other substrata, including floating buoys (Thiel et al. 2003) (Table 13). Along the Chilean coast the solitary ascidian Pyura chilensis may form dense masses on buoys from aquaculture installations — during storms buoys may become detached and float with their fouling community at considerable distances from the shore (Thiel et al. 2003).

\section{Chordata (Vertebrata: Amphibia, Reptilia, Mammalia)}

Few direct observations of rafting of terrestrial vertebrates are available and most reports have anecdotal character (Table 14). However, the sum of and similarities between these reports suggest that at least in some species, rafting may occasionally occur, especially after storms when rivers carry large floats of entangled trees to the open sea. In the Caribbean Heatwole et al. (1963, cited in Heatwole \& Levins 1972) found strong circumstantial evidence for rafting of the toad Bufo marinus from Puerto Rico to Cayo Santiago. Fairbridge \& Teichert (1948, cited in Emery 1955) reported on floating tree trunks on which live frogs had rafted to Low Isles of the Great Barrier Reef.

Some sea turtles frequently associate with floating items. Carr \& Meylan (1980) reported juvenile green and loggerhead turtles among patches of floating Sargassum. While Witherington (2002) stated that these turtles do not raft, but are rather passively aggregated around flotsam where they find good feeding conditions, Fletemeyer (1978) explicitly mentioned neonate loggerhead turtles to raft on a float of Sargassum.

Barbour (1916) mentioned that the snake Boa constrictor had repeatedly been carried from the Orinoco delta to the Caribbean island St. Vincent. Furthermore, he agreed with Matthew (1915) that skinks and gekkos might have been rafting between landmasses. Also Pole (1994) reporting on the biogeography of New Zealand, emphasised the dispersal potential of reptiles, apparently aided by 


\section{MARTIN THIEL \& LARS GUTOW}

Table 14 Vertebrata (Mammalia, Amphibia and Reptilia) reported or inferred as rafting

\begin{tabular}{|c|c|c|c|c|}
\hline Species & Region & $\begin{array}{l}\text { Rafting } \\
\text { inference }\end{array}$ & $\begin{array}{l}\text { Floating } \\
\text { substratum }\end{array}$ & Reference \\
\hline \multicolumn{5}{|l|}{ Amphibia } \\
\hline Bufo marinus & Puerto Rico & $\mathrm{R}$ & $\mathrm{O}$ & $\begin{array}{l}\text { Heatwole et al. } 1963 \text { cited in } \\
\text { Heatwole \& Levins } 1972\end{array}$ \\
\hline Eleutherodactylus planirostris & Florida & $\mathrm{D}$ & n.i. & Meshaka, Jr. et al. 2004 \\
\hline Osteopilus septentrionalis & Florida & $\mathrm{D}$ & n.i. & Meshaka, Jr. et al. 2004 \\
\hline \multicolumn{5}{|l|}{ Reptilia } \\
\hline Anolis sagrei & Caribbean & G & n.i. & Calsbeek \& Smith 2003 \\
\hline Caretta caretta & North Atlantic & $\mathrm{B}, \mathrm{R}$ & M & $\begin{array}{l}\text { Caldwell 1969, Smith 1968, both } \\
\text { cited in Fletemeyer 1978, } \\
\text { Fletemeyer } 1978\end{array}$ \\
\hline Chelonia mydas & Florida & $\mathrm{B}$ & M & Carr \& Meylan 1980* \\
\hline Constrictor constrictor & $\begin{array}{l}\text { St. Vincent } \\
\text { (Lesser Antilles) }\end{array}$ & $\mathrm{B}$ & $\mathrm{W}$ & Guilding 1828, cited in King 1962 \\
\hline Crocodylus intermedius & Barbados & $\mathrm{R}$ & W & Feilden 1889, cited in King 1962 \\
\hline Crotalus adamanteus & Florida & $\mathrm{R}$ & $\mathrm{O}$ & Clench 1925, cited in King 1962 \\
\hline Emoia atrocostata & Krakatau Islands & $\mathrm{C}$ & n.i. & Thornton 1997 \\
\hline Eutropis (Mabuya) multifasciata & Krakatau Islands & $\mathrm{B}$ & $\mathrm{W}$ & Thornton 1997 \\
\hline Furcifer cephalolepis & Comoros Islands & G & $\mathrm{W}$ & Raxworthy et al. 2002 \\
\hline Furcifer pardalis & $\begin{array}{l}\text { Madagascar \& } \\
\text { Reunion Island }\end{array}$ & G & $\mathrm{W}$ & Raxworthy et al. 2002 \\
\hline Furcifer polleni & $\begin{array}{l}\text { Moheli \& Grand } \\
\text { Comoro }\end{array}$ & G & $\mathrm{W}$ & Raxworthy et al. 2002 \\
\hline Gekko monarchus & Krakatau Islands & $\mathrm{D}$ & $\mathrm{W}$ & Thornton 1997 \\
\hline Hemidactylus frenatus & Krakatau Islands & $\mathrm{D}$ & $\mathrm{W}$ & Thornton 1997 \\
\hline Hemiphyllodactylus typus & Krakatau Islands & $\mathrm{D}$ & $\mathrm{W}$ & Thornton 1997 \\
\hline Iguana iguana & Lesser Antilles & B & $\mathrm{W}$ & Censky et al. 1998 \\
\hline Mabuya sp. & Africa to America & G & n.i. & Carranza \& Arnold 2003 \\
\hline Tarentola sp. & North Atlantic & G & W & Carranza et al. 2000 \\
\hline \multicolumn{5}{|l|}{ Mammalia } \\
\hline Lepus californicus & California & $\mathrm{R}$ & M & Prescott 1959, cited in King 1962 \\
\hline Perumyscus eremicus avius & Sea of Cortéz & G & n.i. & Hafner et al. 2001 \\
\hline
\end{tabular}

Notes: $\mathrm{B}=$ stranded floating item on beach; $\mathrm{C}=$ circumstantial; $\mathrm{D}=$ distributional inference; $\mathrm{G}=$ genetic inference; $\mathrm{M}=$ macroalgae; n.i. $=$ no information; $\mathrm{O}=$ other; $\mathrm{R}=$ rafting; $\mathrm{W}=$ wood.

* References refer to this species under a different name.

their rafting capabilities. Censky et al. (1998) reported on the arrival of green iguanas (Iguana iguana) on a beach of the Caribbean island Anguilla. Apparently fishermen witnessed the arrival of a large patch of logs and uprooted trees on which the iguanas had travelled. Previous to this event, Anguilla did not harbour green iguanas. To our knowledge this is one of the very few published testimonies of an arrival event of rafting organisms at a new habitat. Similarly, dispersal of chameleons from Madagascar to other areas, particularly to volcanic islands, probably happened passively, by rafting on trees uprooted by storms, or on pieces of land detached by coastal erosion (Rieppel 2002).

Based on phylogeographic relationships between white-footed mice on Islands in the Sea of Cortéz, Hafner et al. (2001) inferred that some species may have been dispersed via rafting. Using 


\section{RAFTING OF BENTHIC MARINE ORGANISMS}

distributional evidence, Brandon-Jones (1998) inferred that primate species that inhabit islands in southeast Asia have reached these via rafting. This author cited various sources reporting on floating islands sufficiently large to carry large mammals (see also Van Duzer 2004).

Rouch (1954) reported an encounter with a floating island north of the Antilles. Since the ship passed the mass of floating terrestrial plants at a considerable distance, an animal clinging to the flotsam was not unequivocally identifiable. Some of the passengers on the ship identified it as a squirrel while others mentioned a small monkey. Additionally, the author cited Darwin, who saw horses and cows on a large floating island.

Genetic studies have also provided support for the inference that terrestrial vertebrates may occasionally be dispersed via rafting (Raxworthy et al. 2002, Calsbeek \& Smith 2003). Even though direct observations of rafting vertebrates are scant, presently available evidence suggests that rafting may have played an important role in dispersal of some species.

\section{Chordata (Vertebrata, Pisces)}

Fishes are common visitors of rafts throughout the oceans (Hunter \& Mitchell 1967, Mitchell \& Hunter 1970, Castro et al. 2002) but most species do not depend on floating items. Fish predation can strongly affect the rafting community and in the present review we focus primarily on this topic. Densities of fishes are often higher around floating objects than in the adjacent open water (e.g., Kingsford 1992, Druce \& Kingsford 1995, Moser et al. 1998, Nelson 2003). Fish have been reported from all types of floating substrata such as tree trunks (Hunter \& Mitchell 1967), macroalgae (Gorelova \& Fedoryako 1986, Kingsford 1992) and plastic items (Nelson 2003). In general, diversity of the fish community is higher below macroalgae than below other floating items (Fedoryako 1989). Even small floating algal pieces of only $5 \mathrm{~g}$ have been found to influence the distribution of pelagic fish (Kingsford \& Choat 1985, 1986). Some fish depend on the raft for spawning or for development of juvenile stages (e.g., Kingsford 1993, 1995, Deudero \& MoralesNin 2000, Ingólfsson \& Kristjánsson 2002). Hentschel (1922) reported fish eggs from patches of Sargassum collected in the Sargasso Sea, and also other authors observed fish eggs attached to floating items (Castro et al. 2002). There may also be regional differences in the colonisation of floating items. Mitchell \& Hunter (1970) found many fish associated with floating macroalgae Macrocystis pyrifera but not with other floating debris (wood planks, plastic crates, etc.) off the California coast. In contrast, in tropical waters fish apparently associated with any floating item (Hunter \& Mitchell 1967, Zarate-Villafranco \& Ortega-García 2000).

Fishes observed around floating algae show three types of associative behaviours (Hirosaki 1960). They may (1) stay almost exclusively within the branches and blades of the algae, (2) remain underneath the floating algae patch or (3) swim around the patch without close association. The fish community under floating items may comprise a wide diversity of species and size ranges (e.g., Klima \& Wickham 1971, Moser et al. 1998). Individuals of small fish species captured from below floating algae are often larger than conspecifics from open waters (Kingsford 1992). In a recent review $>300$ fish species from 96 families were mentioned to associate at least occasionally with floating items (Castro et al. 2002). The majority of these species have been reported as juveniles. In New Zealand waters the occurrence and the proportional representation of small fish species varies seasonally (Kingsford 1992). Many species that are found associated with floating algae are rarely encountered in open waters (Kingsford 1992). Colonisation of floating items may occur rapidly, i.e., within a few hours (Klima \& Wickham 1971, Kingsford 1992, Druce \& Kingsford 1995). Many small fish species and juvenile stages (including larvae) feed primarily on raft fauna (Daborn \& Gregory 1983, Davenport \& Rees 1993, Ingólfsson \& Kristjánsson 2002) while others prey on small fish hiding below rafts (Gorelova \& Fedoryako 1986). Members of this latter group 


\section{MARTIN THIEL \& LARS GUTOW}

also frequently feed on planktonic organisms and raft-associated food items appear to represent opportunistic prey items (Gorelova \& Fedoryako 1986).

Large fish may utilise floating items as meeting points where swarm-fishes associate after feeding excursions at night (see extensive discussion in Castro et al. 2002). Other authors suggested that fish associate under floating items for spawning, since larval transport might be improved in these areas and furthermore larvae and fish might benefit from the accumulation of planktonic food in areas where floating items are concentrated (convergence zones).

\section{Feeding biology of rafting biota}

Sustaining organisms during a long journey requires resources. Not only animal consumers but also many autotrophic organisms, both micro- and macroalgae, live and travel on floating substrata. In general growth conditions for substratum-dwelling algae may be better than for free-living stages or species, since animals co-occurring on the respective floating item may provide nutrients for algae by releasing N and P (Culliney 1970, Deudero \& Morales-Nin 2001a). However, other authors suggested that during open-ocean voyages, nutrient conditions for floating or rafting algae might be limiting since they travel for a long time in the same water body and nutrients may become exhausted (Edgar 1987). While this topic requires attention in the future, it can be generalised that both floating and rafting algae continue to grow during the rafting journey and constitute a continuously replenished food resource for some rafting animals.

Among rafting animals, species with low food requirements, generalist feeders and species that feed on renewable resources are expected to be favoured. Suspension feeders are the dominant feeding group among the organisms reported as rafters, followed by grazers/borers and predators/scavengers (Figure 4). The high proportion of suspension feeders among the rafting fauna is not surprising since their food resources are frequently supplied, in particular when rafting items are concentrated in convergence zones where also suspended materials are enriched (Kingsford \& Choat 1986, Jokiel 1990a, Shanks 2002). Based on stomach analysis and aquarium observations, various studies confirmed that many rafting suspension feeders indeed feed on pelagic organisms such as microalgae or zooplankton (Hentschel 1922, Geiselmann 1983, Tsikhon-Lukanina et al. 2001a, Sano et al. 2003). Growth rates reported for some rafting suspension feeders suggest that the rafting environment indeed supports abundant food resources for these organisms (see below).

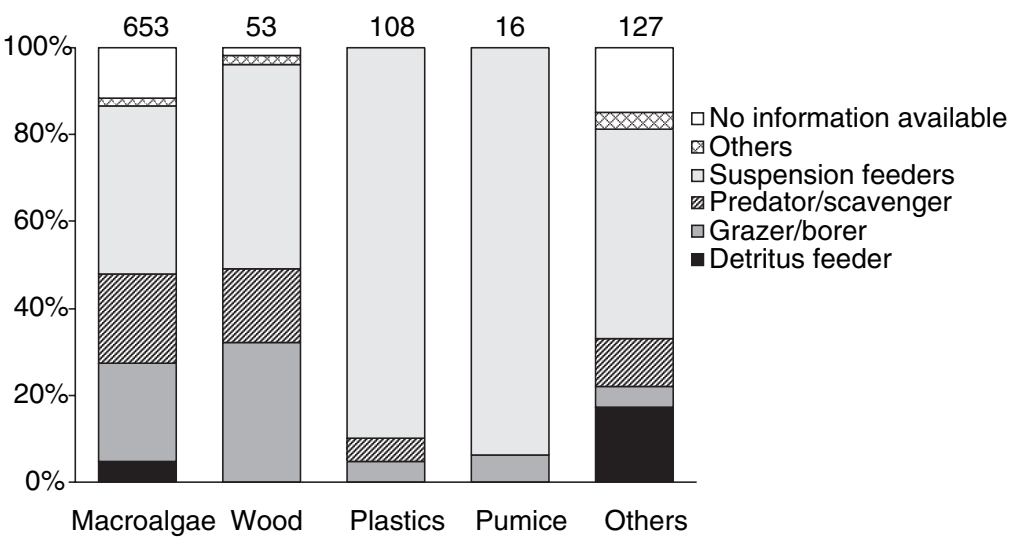

Figure 4 Proportions of rafting marine invertebrates (metazoans) with the respective feeding types reported from the main floating substrata. Numbers on top of columns indicate total number of species reported from each substratum. 


\section{RAFTING OF BENTHIC MARINE ORGANISMS}

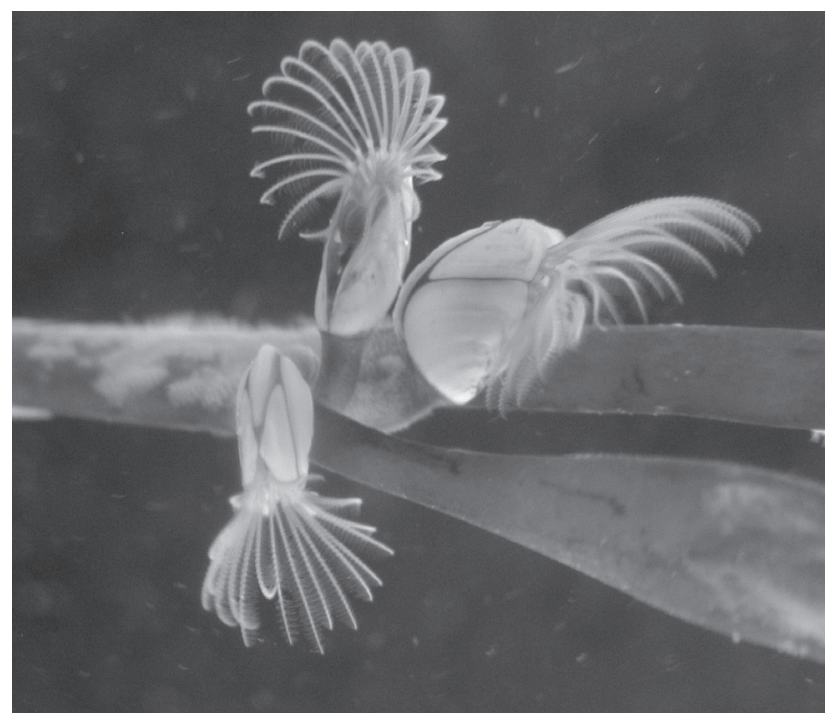

Figure 5 A typical suspension-feeding organism on floating items. Several individuals of the obligate rafter Lepas anatifera are seen from a patch of floating Macrocystis integrifolia found near Coquimbo, Chile.

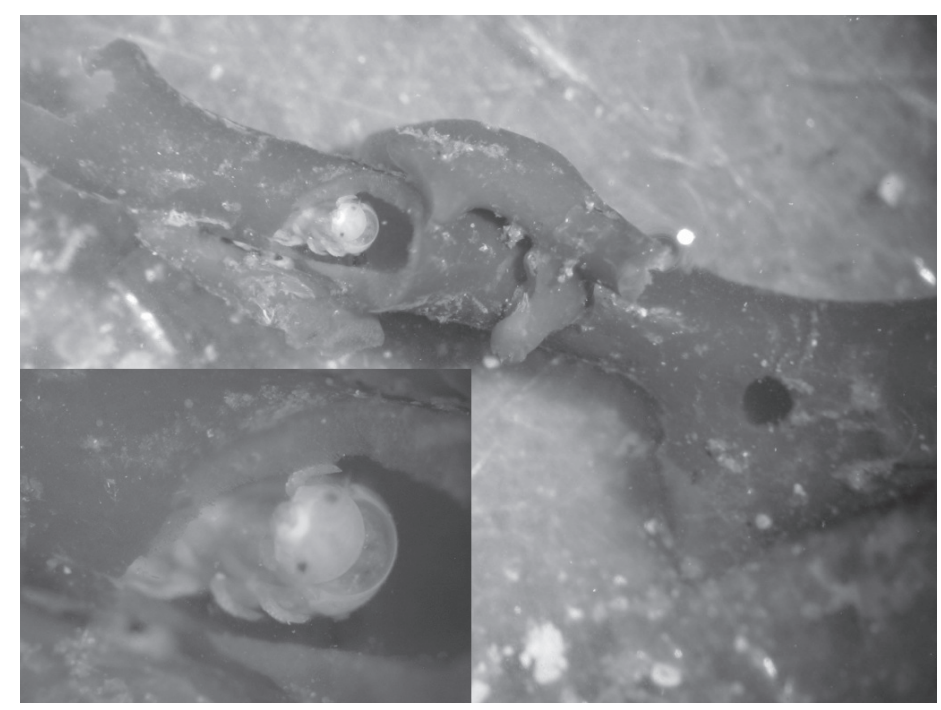

Figure 6 Adult individual of the boring isopod Limnoria chilensis, which is frequently found in holdfasts of the large macroalgae Macrocystis pyrifera and Durvillaea antarctica.

Stalked barnacles (Figure 5) may grow very fast and achieve sexual maturity within weeks after settlement (Skerman 1958, Tsikhon-Lukanina et al. 2001b).

Grazing and boring organisms mostly have been found on floating wood and macroalgae, but some species are also reported for abiotic substrata (Figure 4), where they probably feed on rafting algae. In contrast to suspension feeders, grazing and boring species actively contribute to the destruction of their rafting substrata (Figure 6). Several authors who experimentally explored rafting substrata reported on the destructive activity of grazing and boring rafters (Edgar 1987, Vásquez 1993). Nevertheless, the high abundances of grazers and borers reported for some floating items 


\section{MARTIN THIEL \& LARS GUTOW}

(e.g., Ingólfsson 1998, Hobday 2000a) demonstrate that these organisms at least temporarily can raft successfully.

Predators and scavengers are also commonly found as rafting organisms. Cheng (1975) had already remarked on the ubiquity of predatory species in the pleuston community. Possibly, the predators and scavengers among the rafting fauna benefit from the passive accumulation of zooplankton and animal carcasses in convergence zones where floating items also abound. Zaitsev (1970) coined the term 'anti-rain' for recently dead small zooplankters which, instead of sinking, rise to the sea surface where they are then concentrated in convergence zones by surface currents. Postmortem rise to the sea surface has also been suggested for larger dead invertebrates, such as Nautilus carcasses (Chamberlain et al. 1981). Some of the motile obligate rafters such as Planes minutus and Idotea metallica appear to be highly plastic in their feeding ecology and are reported as predators and scavengers by some authors (Davenport 1992, Gutow 2003a), while other authors found primarily algae in stomach contents of these species (Sano et al. 2003). These observations indicate that generalist feeders are favoured among the rafting fauna (see also below). Also many fish predators associating with floating objects have a wide food spectrum (Anraku \& Azeta 1967, Ida et al. 1967, Gorelova \& Fedoryako 1986, Shaffer et al. 1995, Deudero \& Morales-Nin 2001b).

A wide diversity of detritus feeders is also reported from floating items. Most of these organisms probably feed on algal remains that are either continuously supplied from benthic coastal habitats or that are released from floating algae themselves. Low proportions of certain feeding groups on floating items, such as found for deposit feeders, suggest that some organisms do not do well in the rafting environment. In this context Scheltema (1977) remarked that "it is fanciful to believe that benthic infaunal deposit feeders will have the slightest chance for dispersal by rafting". Myers (1993) also expressed that sediment-dwelling amphipods are unlikely candidates for rafting dispersal. While in general these statements are true, the odd exceptions are not lacking: some deposit-feeding polychaetes and ophiuroids have been reported from algal holdfasts or dense algal mats (Averincev 1980, Highsmith 1985), where they probably dwell in accumulated sediments.

Besides these organisms there are several symbiont species reported from floating items. Some of these are parasites feeding on their hosts (e.g., bopyrid crustaceans), while others are commensals. Again others can be considered as slow decomposers of organic matter, e.g., arenicolous fungi on driftwood.

In general, two main categories of rafting organisms can be distinguished, those that do not rely on their floating substratum as food resource (autotrophic organisms, suspension feeders and predators) and those that actively consume their raft (grazers, borers and scavengers). Animals from the first category exploit allochthonous resources, while those from the second category mainly feed on autochthonous food items. Interestingly, the first category comprises a comparatively large number of cosmopolitan species. This suggests a possible link between feeding mode and dispersal potential. Species that do not rely on their raft as food resource may leave their rafts intact and colonise floating items resistant to biotic destruction, and thereby achieve farther dispersal distances than species that consume their rafts during the voyage.

\section{Reproductive biology of rafting biota}

The fact that reproductive biology has important implications for the success of long-distance rafters had been recognised early on. Many workers have remarked on the high proportion of species with direct development in regions far from potential source regions. This has been termed the 'Rockall' paradox by Johannesson (1988) who contrasted the geographic range of a littorinid gastropod with pelagic larvae with that of a congeneric species with direct development. She reported that the species that releases fully developed juveniles (Littorina saxatilis) has a much wider geographic distribution than the species with pelagic larvae (L. littorea). Littorina saxatilis even occurred on 


\section{RAFTING OF BENTHIC MARINE ORGANISMS}

remote islands where L. littorea was absent. Other authors examining the fauna on remote islands or seamounts also observed a surprisingly high proportion of species with direct development (Leal \& Bouchet 1991, Parker \& Tunnicliffe 1994, Davenport \& Stevenson 1998) and inferred rafting as the most likely dispersal mechanism responsible for these patterns. Several authors who collected rafting fauna from floating macroalgae also remarked on the high proportion of species with direct development (Hobday 2000a) or other reproductive strategies apparently favourable for rafters (e.g., asexual reproduction, Jackson 1986).

Given that on a floating item only limited amounts of space and food are available, species that reproduce at small sizes would be favoured (Highsmith 1985). Stalked barnacles can reproduce at small size (Tsikhon-Lukanina et al. 2001a). In this context it is also interesting to note that Hentschel (1922) remarked that hydrozoans growing on floating Sargassum are comparatively small. Similarly, Timmermann (1932) observed that pycnogonids collected from floating Sargassum reached sexual maturity at substantially smaller sizes than conspecifics from coastal waters. He was aware of the fact that these rafting populations may be isolated from coastal populations and represent separate species. Regardless of the reasons for predominance of small body size among rafters, it should be kept in mind that small organisms have a limited capacity of producing large numbers of gametes and therefore often feature internal fertilisation and offspring brooding (Strathmann \& Strathmann 1982).

Many rafters frequently reproduce during or immediately after finishing their journey. Rafters either may embark on floating items as sexually mature adults or they may achieve adulthood during rafting. As becomes evident from the above considerations, several reproductive features may be advantageous for rafting organisms. For example, species that reproduce asexually can start to reproduce on a floating item whenever environmental conditions allow. Few rafters exclusively reproduce asexually but many species feature a combination of asexual and sexual reproduction (Table 15). Asexual reproduction appears to be primarily important for hydrozoans and byrozoans growing on macroalgae (Hentschel 1922, Ryland 1974, Cornelius 1992a) and other floating items (Winston 1982). Under suitable conditions these species may completely dominate the rafting community (e.g., Hentschel 1922). Anthozoans and ascidians that are found rafting may also reproduce asexually (Vallentin 1895, Hirosaki 1964, Bushing 1994). Asexual production of planula larvae has been demonstrated for the coral Pocillopora damicornis (Stoddart 1983). Coulson et al. (2002) discussed that several mites and collembolans reproduce parthenogenetically, and that one single individual would suffice to establish a population after reaching new shores (see also Moore 2002).

In species with sexual reproduction and cross-fertilisation the presence of members of both sexes is required for successful fertilisation. Some of the organisms reported as rafters feature external fertilisation, e.g., the bivalves Aulacomya ater and Mytilus edulis (Arnaud et al. 1976, Ingólfsson 1992, Castilla \& Guiñez 2000). However, fertilisation success of external fertilisers

Table 15 Number of rafting marine invertebrate species with the respective sexual system

\begin{tabular}{lc}
\hline Sexual system & No. of species \\
\hline Hermaphroditic & 99 \\
Gonochoric & 235 \\
Asexual & 3 \\
Combined (asexual \& sexual) & 117 \\
No information available & 495 \\
\hline
\end{tabular}




\section{MARTIN THIEL \& LARS GUTOW}

depends on high concentrations of gametes, which can only be achieved in the presence of many gamete-releasing conspecifics (Wahle \& Gilbert 2002). Most floating items have a limited carrying capacity and consequently may not be a suitable reproductive environment for species with external fertilisation. On the other hand, floating items typically accumulate in convergence zones where also gametes may become concentrated. These hydrographic conditions could then facilitate successful fertilisation of gametes released by rafting species with external fertilisation. High densities of larvae (of typical rafters) in convergence zones (e.g., Shanks 2002) could thus partly be supplied by successful reproduction of rafting organisms. However, despite potentially favourable hydrographic conditions few rafting organisms feature external fertilisation (4 of 368 species for which information about fertilisation mode was available). This is probably due to the comparatively low densities and small body sizes of typical rafters, resulting in gamete concentrations too low for successful fertilisation.

Internal fertilisation, which appears to be most common among rafting species (364 species, i.e., $98.9 \%$ of the species for which information about fertilisation mode was available), usually requires direct contact between a male and a female. Species with elaborate courtship may not be favoured on floating items since neither space nor substratum characteristics may allow for such mating behaviours to take place. Mating in a tube or in a nest may be easier than mating on the open surface of a floating item. Little is known, though, about the mating behaviour of rafting organisms. Following mating, females may store sperm for future reproduction, which appears particularly favourable in situations where encounters between the sexes are unpredictable. Indeed, for some rafting species it is known that females store sperm for future fertilisations. This has been reported for most turbellarians (Brusca \& Brusca 2003), a marine mesogastropod (Littorina saxatilis, Wilhelmsen 1999), a marine neogastropod (Nucella emarginata, Marko 1998), many nudibranchs (Brusca \& Brusca 2003) and several chameleon species for which rafting has been inferred based on their phylogeography (Furcifer cephalolepis and F. pardalis, Raxworthy et al. 2002). Also for Anolis lizards, which are thought to be frequently dispersed between Caribbean Islands via rafting (Calsbeek \& Smith 2003), it is known that females can store sperm for several months (Orrell \& Jenssen 2002). Sperm storage is generally widespread among reptiles (Birkhead \& Moller 1993, Olsson \& Madsen 1998, Sever \& Hamlett 2002), and also parthenogenetic reproduction is known in some genera (Reeder et al. 2002), rendering members of this taxon particular suitable for successful colonisation of new habitats.

Jokiel (1984) remarked that the coral Pocillopora damicornis and some other coral species may be self-fertile. Several other hermaphroditic rafters (Jokiel 1989, Ó Foighil 1989, Byrne 1995, Edmands \& Potts 1997, Winston et al. 1997) and some floating macroalgae (Deysher \& Norton 1981) have been reported to feature self-fertilisation, another reproductive trait that renders gamete exchange with members of the opposite sex unnecessary. Even though presently only few rafting species are known to be self-fertile, the capability to sexually produce offspring without the immediate need of sexual partners appears to be an important advantage for rafting organisms. It can be hypothesised that species that require the presence of mates for reproduction must occur in sufficient densities to achieve successful fertilisation.

After successful fertilisation has been accomplished, developing embryos may be incubated in or on the parent's body, deposited in special structures on the substratum, or released into the water column. Many rafting organisms incubate their developing embryos for some time before releasing them (Table 16). Brooding of embryos on or in the parental body occurs in rafting molluscs (e.g., Ó Foighil 1989, Helmuth et al. 1994, Johannesson 2002), echinoderms (Mortensen 1933, Fell 1967, Sponer \& Roy 2002), polychaetes (Knight-Jones et al. 1972), crustaceans (Edgar 1987, Ingólfsson 1995, 1998, 2000, Gutow \& Franke 2003), bryozoans and tunicates (Brusca \& Brusca 2003). Many terrestrial rafters also incubate their offspring. King (1962) reported a gravid snake containing many young ones. 


\section{RAFTING OF BENTHIC MARINE ORGANISMS}

Table 16 Number of rafting marine invertebrate species with the respective developmental modes

\begin{tabular}{lc}
\hline Developmental mode & No. of species \\
\hline Pelagic larvae & 90 \\
Benthic or very short pelagic larvae & 83 \\
Egg masses: pelagic larvae & 10 \\
Egg masses: fully developed juveniles & 7 \\
Direct: fully developed juveniles & 193 \\
No information available & 566 \\
\hline
\end{tabular}

Some of the most common marine invertebrates reported rafting are peracarid crustaceans (amphipods, isopods and tanaids), all of which incubate their embryos in a marsupium. Peracarids release fully developed juveniles from the marsupium and in many species females provide extended parental care for their offspring (Thiel 2003a). In these latter species, females host juveniles in their burrows, and juveniles are known to recruit in the immediate vicinity of their parents (Chess 1993, Thiel 2003b). This form of local recruitment can be advantageous for rafting organisms, since local populations can be maintained on a floating item, even when the duration of the journey exceeds the typical lifetime of a species. Referring to spirorbid polychaetes growing on macroalgae, Knight-Jones et al. (1975) mentioned that "in such epiphytic spirorbids a considerable proportion of the larvae may settle without a pelagic stage (Gee 1963), so populations are easily built up and might conceivably be maintained on growing, drifting plants during ocean passages lasting several years". The fact that offspring of spirorbid polychaetes recruit near the parent had already been noticed by Hentschel (1922). Bushing (1994) also noted that brooded subadults of Pycnogonum stearnsi were found on floating macroalgae, implying that these were released on and recruited within algae during the journey. Observations by Helmuth et al. (1994) on the brooding bivalve Gaimardia trapesina also suggest that offspring can recruit within the parental raft. To our present knowledge strongest indication for local recruitment comes from peracarid crustaceans (Tanaka \& Leite 2003) but there is good reason to believe that this may also occur in other species with direct development. Support comes from community and also from genetic studies (see below).

Many rafting organisms also deposit their developing offspring in brood structures on the substratum. This is most common among gastropods. Bushing (1994) reported that rafting individuals of several nudibranch species were observed to lay eggs on floating macroalgae. Worcester (1994) reported egg masses of Phyllaplysia taylori and other opisthobranch species on floating seagrass blades. The wide geographic distribution of some large gastropods without pelagic larvae might be attributed to the floating capability of their egg capsules (Marche-Marchad 1968). Large communal egg masses from at least 10 females of the gastropod Nassarius reticulatus have been recorded from a plastic bag found at the Dutch island of Texel (Cadée 1999). Since the species is rare in that region the egg masses probably arrived via rafting from more southerly source populations. Egg masses from several other gastropod species with direct development have been reported from floating substrata (Vallentin 1895, Ockelmann \& Nielsen 1981, Scheltema 1995, Kyle \& Boulding 2000). Hentschel (1922) found several different egg masses, some of which he attributed to gastropods and some to rafting turbellarians. Some fish species associated with floating items also deposit their eggs onto these (Dooley 1972), but after hatching further development occurs in the water column. In those species that lay eggs on floating substrata, rafting may occur even in the absence of adults.

Several authors also inferred for terrestrial arthropods that dispersal might be achieved via eggs or dormant stages, which are more resistant to sea water than are adults. For example, Pugh (2004) 


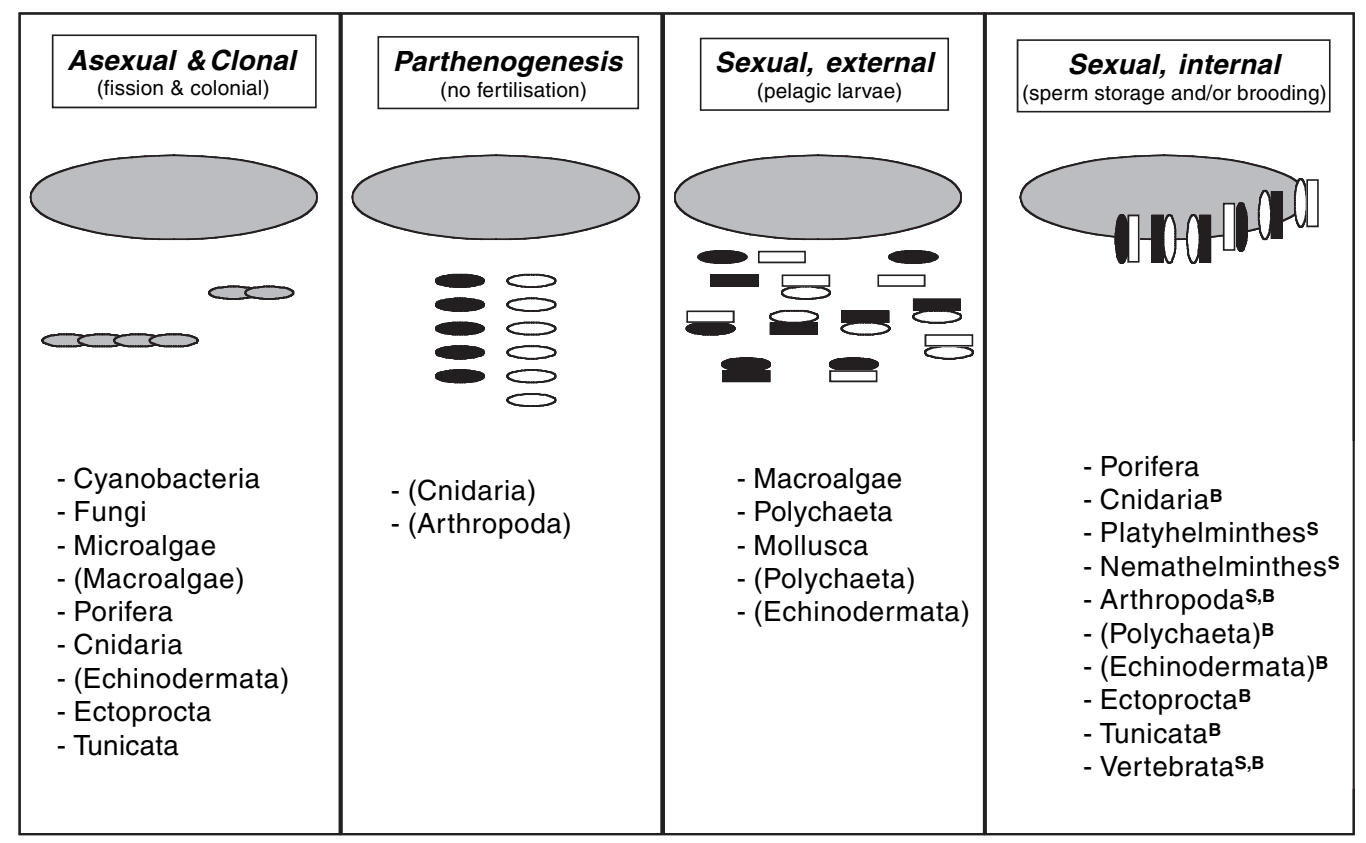

Figure 7 Schematic presentation of main reproductive traits reported from rafting organisms and taxa from which the respective traits are known. Propagules can result either from asexual mitosis (grey) or from meiotically produced gametes (black and white). Superscripts denote B = Brooding and $\mathrm{S}=$ Sperm storage.

suggested that the wide distribution of certain spiders could be the consequence of rafting dispersal of eggs or cocoons on trees. Mites from the genus Euptyctima also seem well adapted for rafting since their eggs are laid in dead wood wherein the developing larvae further bore tunnels (Niedbala 1998). Thornton (1997) also remarked that eggs, larvae or pupae of wood-dwelling insects, which probably were transported via driftwood, were among the first colonisers of Krakatau.

Some rafting organisms also release their larvae into the water column. Many bryozoan species from floating plastics release pelagic larvae (Winston et al. 1997) but these typically are short-lived (Stevens et al. 1996). The commonly observed high recruitment success of stalked barnacles on floating items is possibly because both larvae and floating items become concentrated in convergence zones (Kingsford \& Choat 1986, Shanks 2002). Release of pelagic propagules may also be advantageous for rafters when these return to coastal habitats after a long oceanic voyage (see below).

In summary, certain reproductive traits predominate among rafting organisms, mainly asexual reproduction, internal fertilisation and direct development (Figure 7). These traits will be particularly advantageous for long-distance dispersal, since they permit the persistence of local populations via local reproduction, even when floating duration exceeds the typical lifetime of rafters. Under such circumstances it is not the original travellers but rather their offspring that arrive in new habitats.

\section{Growth of rafting organisms}

For most facultative rafters, the environmental conditions on a floating item are very different from those in their native habitats. The relatively oligotrophic rafting environment in the open ocean may not provide sufficient food resources to sustain large organisms over long time periods. In contrast, small organisms may obtain enough food on floating substrata to grow and reproduce 


\section{RAFTING OF BENTHIC MARINE ORGANISMS}

successfully, which is considered important in maintaining persistent local populations during a long journey (see above). Hentschel (1922) and Timmermann (1932) suggested that restrictions imposed on organisms by food limitations may have resulted in the comparatively small body size of common rafters from the Sargasso Sea. In this context a comment from Carpenter (1970) on attached diatoms from Sargassum is noticeable; he observed that specimens from Sargassum were smaller than conspecifics from nearshore areas and suggested that this reduction in size could be a consequence of relatively low nutrient concentrations in the Sargasso Sea. This effect might be conserved throughout the food chain finally affecting the size of heterotrophic rafters. Certain nematode species are found to be considerably smaller on floating Sargassum than on conspecifics from benthic habitats (Micoletzky 1922). Thus, many rafting organisms experience restrictions in growth and adult body size on floating items.

However, growth conditions in the rafting environment appear favourable for some species. Hentschel (1922) emphasised that guts of common rafters from Sargassum are often completely filled, indicating a sufficient food supply. Also the fact that juvenile stages of many fishes associate with floating items where they feed intensively and gain protection from predators shows that conditions in the rafting environment are suitable for their growth.

While there are many qualitative observations that indicate fast growth of some rafters, quantitative growth estimates for typical rafters are few or come from laboratory experiments. In some cases, floating items have been deployed at well known dates (i.e., tethered items or buoys and pumice introduced to the sea) and by using reasonable assumptions about first colonisation of these items, growth rates for rafting organisms have been obtained. The best growth estimates are available for stalked barnacles (Table 17). In most species studied to date, growth is rapid and individuals achieve sexual maturity within a few weeks after settlement. Patel (1959) observed that moulting frequency in Lepas anatifera is temperature dependent, with the highest frequency in the range of the assumed temperature optimum $\left(17-27^{\circ} \mathrm{C}\right)$. In general, suspension feeders that consume a wide variety of suspended food particles, including small zooplankters, will find optimal growth conditions on floating items. This is confirmed by the high growth rates reported for stalked barnacles and also by the observations that hydroids and bryozoans quickly overgrow floating substrata (Ye \& Andrady 1991, Tsikhon-Lukanina et al. 2001a).

Shipworms (Bivalvia, Teredinidae), which can be found in a wide variety of wood (Nair \& Saraswathy 1971), may also grow rapidly when colonising suitable substrata. Edmondson (1962) reported high growth rates for teredinid bivalves, measuring a burrow depth of $75 \mathrm{~mm}$ in 140 days $\left(=0.54 \mathrm{~mm} \mathrm{~d}^{-1}\right)$ for Teredo gregoryi. For Lyrodus pedicellatus Gallager et al. (1981) estimated a mean growth rate of $0.59 \mathrm{~mm} \mathrm{~d}^{-1}$, which they consider a typical value for teredinids. Nair \& Saraswathy (1971) emphasised that growth rates depend, among other factors, on temperature and substratum characteristics.

Also some grazers that are well adapted to the rafting environment may grow fast on floating items. Based on laboratory experiments, Gutow (2003b) reported average growth rates of $0.37 \mathrm{~mm}$ $\mathrm{d}^{-1}$ for Idotea metallica and $0.28 \mathrm{~mm} \mathrm{~d}^{-1}$ for I. baltica. These rates strongly depended on temperature and food quality. When analysing body sizes of adult I. resecata in relation to raft age, Hobday (1998, cited in Hobday 2000a) revealed a significant positive relationship, suggestive of active growth in this species. Several other species analysed by Hobday (2000a), including some decapod and fish predators, showed a similar positive (but non-significant) raft age-size relationship. Predators feeding on common rafting organisms are known to have high growth rates on rafts (e.g., fishes, Shaffer et al. 1995). Under suitable conditions the nudibranch Fiona pinnata grows rapidly and can reach sexual maturity within approximately two weeks after hatching (Il'in \& Roginskaya 1987).

Clearly, these reports on growth rates from some rafters refer to an eclectic species club of organisms well adapted to the rafting environment. Other organisms, which cannot fulfil their food 
MARTIN THIEL \& LARS GUTOW

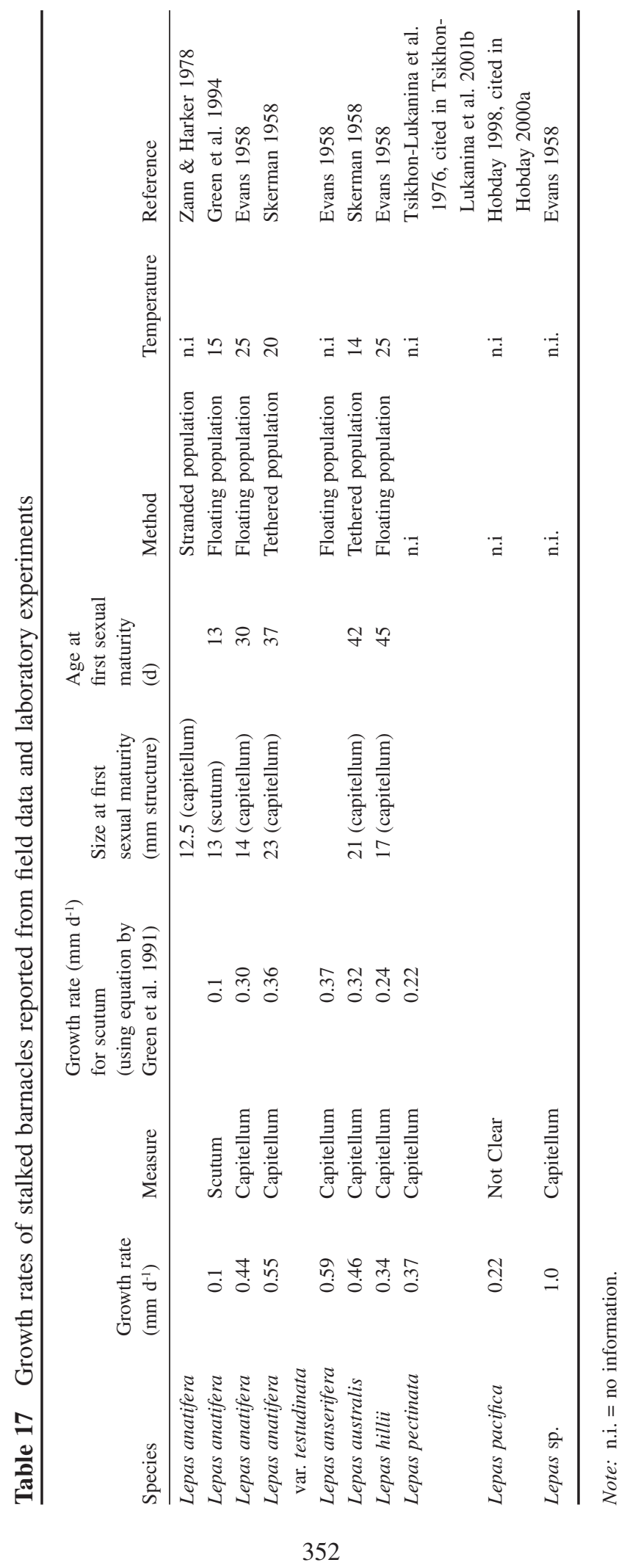




\section{RAFTING OF BENTHIC MARINE ORGANISMS}

requirements on a raft, may have to live on stored resources during a rafting journey and consequently their growth rates will be minimal. While at present the available information is very limited, it can be summarised that organisms capable of active feeding during the rafting journey can achieve high growth rates and successful reproduction, allowing them to rapidly build up local populations on floating items.

\section{Survival and longevity of rafting organisms}

Not all organisms are equally well adapted to survive on floating items. As becomes evident from the preceding sections, some species with a particular feeding biology and favourable reproductive traits are better suited for rafting than others. For example, besides feeding and reproductive biology, body size might be a selective factor during long-distance voyages because small organisms require less food than do large organisms.

A high proportion of rafters, in particular on abiotic floating items such as plastics and volcanic pumice, are sessile organisms (Figure 8). Morton \& Britton (2000) also recognised that many potential rafters have a sessile lifestyle. While the sessile lifestyle confers the advantage of being tightly bound to a floating substratum, this also can turn into a disadvantage. Sessile organisms cannot change their position within a raft, which would be important if the raft turns over during the journey and organisms are exposed to the air, solar radiation and extreme temperatures (Figure 9). Another cost of a sessile lifestyle is the incapacity of a rafting organism to leave its raft, which could become a serious disadvantage when a raft starts to sink or when getting in contact with better rafts or suitable new habitats (see also below). Furthermore, the capacity to leave the raft might be an important trait for the colonisation of a new region after a rafting journey.

Motile rafters can change their position or switch between floating items but most are poor swimmers and they need to cling efficiently to the raft in order not to become separated from their life support. Holmquist (1994), studying drifting seaweeds, observed that many gastropod and crustacean species managed to hang on to seaweeds tumbling over the sea floor. Hanging on is also important for organisms on floating items. Many species may easily fall off floating items as has been reported for algal-dwelling invertebrates by several authors (Kingsford \& Choat 1985, Edgar \& Burton 2000). Possibly it is not by chance that some of the most common motile amphipods on rafts belong to the caprellids (e.g., Thiel et al. 2003), which are particularly well adapted to cling to diverse substrata (Aoki \& Kikuchi 1995, Takeuchi \& Hirano 1996) (Figure 10). Isopods

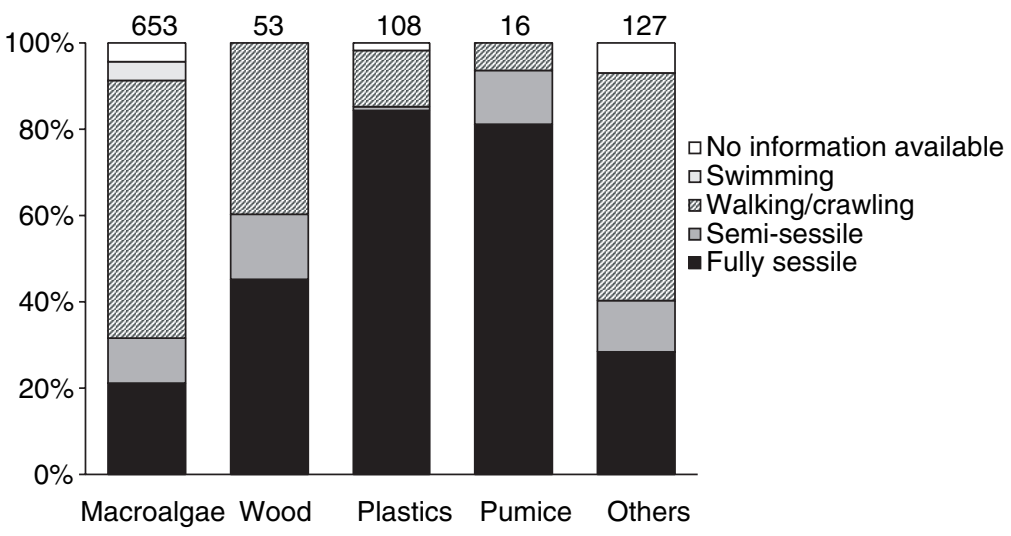

Figure 8 Motility of rafting marine invertebrates (metazoans) found on the respective floating substrata. Numbers on top of columns indicate total number of species reported from each substratum. 


\section{MARTIN THIEL \& LARS GUTOW}

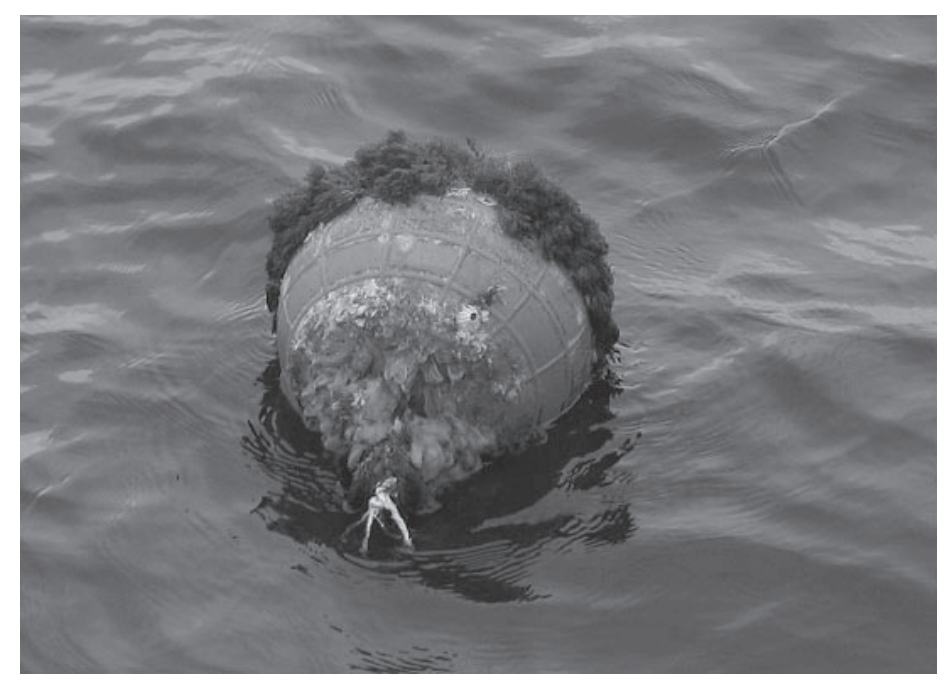

Figure 9 A freely floating buoy that was dislodged from aquaculture installations near Coquimbo, Chile. The buoy harboured a dense assemblage of sessile rafters, many of which were dying, probably due to frequent exposure to air while the buoy was turning around.

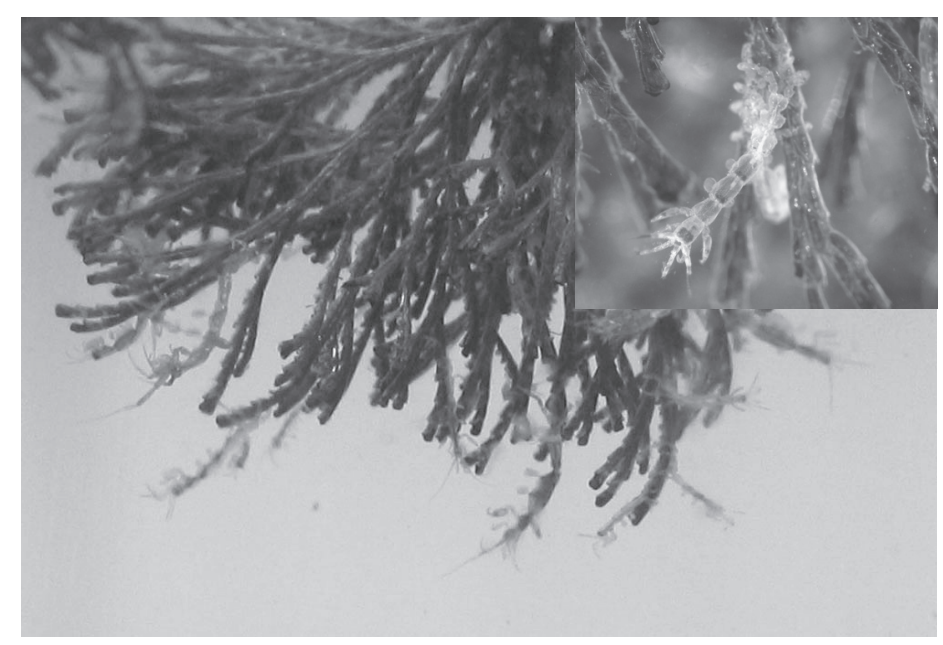

Figure 10 Caprellid amphipods (Caprella verrucosa) clinging efficiently to the underlying substratum, here the erect bryozoan Bugula neritina.

from the genus Idotea, very commonly observed rafting, also cling strongly to floating items. Peracarid crustaceans in general are well known for their good clinging capacity (Vader 1983) but this is also noted for other arthropods, e.g., harpacticoid copepods (Yeatman 1962), decapods (Tanigawa 2001) or pycnogonids (Fage 1949, González \& Edding 1990b). Many bivalves produce byssal threads allowing them to gain efficient hold to the underlying substratum, including floating items (e.g., Highsmith 1985, Ó Foighil 1989, Hayami \& Kase 1996). Efficient caudal glands allow certain nematodes to anchor themselves tightly to algal rafts (Micoletzky 1922). An interesting case was mentioned by Masó et al. (2003) who suggested that the stickiness of temporary cysts of the dinoflagellate Alexandrium taylori may favour passive dispersal of this species on floating plastics. 


\section{RAFTING OF BENTHIC MARINE ORGANISMS}

Other motile organisms hide in burrows and crevices, and here again smallness may be favoured. Species that live in close-fitting burrows seem to be favoured since these burrows allow both a firm hold to the substratum and additionally can be closed off, permitting protection against unfavourable environments. This latter point appears particularly important for terrestrial arthropods, since they may be susceptible to seawater immersion. Terrestrial species bound to a floating item may have to fight against immersion in sea water and lack of freshwater. Barbour (1916) pointed out that “... naked gastropods, earthworms, fresh-water fishes or crayfishes, Peripatus and a host of such delicate creatures simply cannot withstand salt water" emphasising that most terrestrial species are not well adapted to survive the voyage on a raft. For example Monge-Nájera (1995) mentioned that onychophorans, when placed in sea water, quickly die. However, the biogeography of some onychophorans from the Caribbean suggests that rafting may have occurred in the past (MongeNájera 1995). Other terrestrial invertebrates, for example oligochaetes (Wittmann 1934) or tardigrades (McInnes \& Pugh 1998), are similarly ill-adapted for rafting. Among the non-marine molluscs from subantarctic regions only a few species from the family Hydrobiidae appear capable of rafting (Pugh \& Scott 2002). Also, most spiders from that region are not capable of rafting (Pugh 2004). Bayly et al. (2003) discussed that freshwater copepods in general are not very suited for natural dispersal including transport via rafting. Pugh et al. (2002) noted that most non-marine crustaceans from Antarctica and subantarctic Islands are intolerant of seawater dispersal. Interestingly, Peck (1994) reported a relatively diverse non-oceanic crustacean fauna from the Galápagos Islands, and suggested that most species had arrived via rafting. Possibly, the rafting environment in the tropical East Pacific is more benign than in the Southern Oceans. Furthermore, potential landing sites on the Galápagos Islands may present better conditions for successful colonisation than shores in the Southern Oceans.

Several authors emphasised that burrow-dwelling insects may be relatively good rafters (Wheeler 1916, Heatwole \& Levins 1972, Abe 1984, Gathorne-Hardy \& Jones 2000). Heatwole \& Levins (1972) observed that most of the terrestrial insects they recovered from floating items were wood-boring species (beetle larvae and termites) that lived well protected in self-constructed burrows within the wood substratum. Insects and mites living in plant galls had high survival times in sea water (Docters van Leeuwen 1936). Gathorne-Hardy \& Jones (2000) found that most termite species that recolonised Krakatau Islands after the 1883 eruption are wood-living species and they suggested that this habit may provide rafting opportunities and favour survival at sea (see also Thornton 1997). Johansen \& Hytteborn (2001) discussed that seeds and other organisms embedded in the soil held between roots of floating trees might be somewhat protected from seawater contact. Hemsley (1885) reported that he obtained three germinating vascular plants from a small amount of earth enclosed in this manner in a stranded oak tree.

Terrestrial species that are not living in burrows or are protected otherwise need to have some other means of resisting sea water. Levins \& Heatwole (1963) pointed out that abundant coastal species with wide environmental tolerances are most likely to become successful rafters. Most termite species that colonised the Krakatau Islands are considered salt tolerant (Gathorne-Hardy \& Jones 2000). Protection from or tolerance to saltwater contact may not only be of importance for terrestrial arthropods (see, e.g., Coulson et al. 2002), but also for rafting vertebrates. Possibly the fact that some reptiles can withstand conditions on fragile rafts at sea is a consequence of their physiology, being tolerant to sea water, ectotherms and less dependent on freshwater than for example mammals (Carlquist 1965). This idea is supported by Carranza et al. (2000) who discussed that geckos from the genus Tarentola may be pre-adapted to rafting dispersal because of their low food requirements and the fact that they inhabit dry habitats and can go for long periods without freshwater. Tolerance to temperature extremes may also be advantageous for successful rafting. Moore (2002), who inferred that the disjunct distribution of the arctic springtail Tetracanthella 


\section{MARTIN THIEL \& LARS GUTOW}

arctica could be the result of rafting, suggested that the capability to survive extremely low temperatures $\left(-22^{\circ} \mathrm{C}\right)$ for years is conducive to ice-rafting.

At the sea-air interface a diverse cocktail of chemical compounds accumulates (Zaitsev 1970, Cheng 1975, Wurl \& Obbard 2004). Natural oceanic surface films are commonly formed by surfaceactive lipid materials consisting of fatty acids, fatty alcohols and fatty acid esters that originate from marine animals and plants (Morris 1974). Pollutants such as heavy metals, too, accumulate in the upper water layer (Wurl \& Obbard 2004), and are probably introduced into the marine food web when being ingested by surface-dwelling organisms (Morris 1974). Morris et al. (1976), however, agree with Burns \& Teal (1973) that hydrocarbons from petroleum pollution, which are often found associated with floating Sargassum, are accumulated in the organisms primarily across respiratory surfaces. Tar balls have been found to stick to floating Sargassum (Butler 1975). Since hydrocarbon concentrations do not increase from one trophic level to the next, oil pollutants are not concentrated in the Sargassum community through uptake along the food chain (Morris et al. 1976). Discussing possible effects of toxins on floating Sargassum Stoner (1983) mentioned comparable impacts on the associated fauna. Capuzzo (1987, cited in Committee on Oil in the Sea 2003) describes that toxic effects of hydrocarbons on marine organisms include severe impairment of reproduction and feeding mechanisms as well as increased susceptibility to diseases. In addition to hydrocarbons, considerable concentrations of germanium and mercury have been found in shrimps and barnacles from the Sargassum community (Johnson \& Braman 1975).

Organisms living on floating items close to the sea surface may also be exposed to high UV radiation. Some obligate rafters apparently possess UV-shielding pigments. The isopods Idotea metallica and I. stephenseni feature deep blue colouration, interpreted as an adaptation of neuston organisms to high levels of UV radiation (Herring 1969, Zaitsev 1970). The pigmentary system of I. metallica consists of epidermal chromatophores, iridophores and carotenoid pigments (Herring 1969). The latter are mainly obtained in the form of astaxanthin taken from other crustaceans consumed by the isopods. The animals can actively adapt to different light intensities by expansion and contraction of the chromatophores and the iridiphores. Expansion of the iridophoores generates a metallic glimmer, which is characteristic of surface-dwelling plankton organisms. Zaitsev (1970) noted that the neustonic community is typically much richer in pigmented invertebrates than groups of the water column. Many permanent members of the Sargassum community also possess cryptic coloration and mimicry (e.g., Timmermann 1932), permitting better protection from predatory fishes (Crozier 1918, Adams 1960, Hacker \& Madin 1991). Cheng \& Lewin (1975) remarked on the 'beautifully camouflaged' turbellarian Stylochoplana sargassicola found in high abundances among floating Sargassum. Many nudibranchs also match the colour pattern of their principal substratum (Schrödl 1999a). Hitchcock (1941) and Chace (1951) reporting on mimicry of Planes minutus suggested that white patches on the carapace may mimic calcareous tubes of epibiotic polychaetes or bryozoan colonies. Castro et al. (2002) pointed out that some fish associates even assume a colouration similar to that of the floating item, most likely offering better protection from visual predators. An example for this kind of visual protection is the Sargassum fish Histrio histrio, whose colouration and weed-like appendages provide a prime example for this mimicry (Adams 1960). Some of these adaptations have also been reported for facultative rafters, and species that are protected from predators (visually, structurally, chemically or behaviourally) may better survive the high predation pressure in the rafting environment.

\section{Obligate and facultative rafters}

Rafting organisms can be categorised in various ways. Ingólfsson (1995) distinguished four groups of rafters according to their origin, namely species that (1) live in intertidal macroalgae, (2) colonise macroalgae cast ashore, (3) usually live in subtidal habitats and (4) consist of planktonic and 


\section{RAFTING OF BENTHIC MARINE ORGANISMS}

neustonic organisms. Ekman (1967, cited in Adams 1960) and Friedrich (1969) provided corresponding divisions of the Sargassum fauna into four functional groups represented by (1) motile epifauna, (2) sessile epifauna, (3) haptic or clinging fauna and (4) nektonic fauna hiding in or otherwise associated with seaweed. Adams (1960) suggested an additional group termed 'associated organisms' that can be identified in spite of significant overlap with the other groups. Associated organisms may be further subdivided into (1) self-buoyant invertebrates such as the siphonophores Physalia sp. and Velella sp. or the gastropod Janthina sp. that become entangled with floating seaweed caught in the same current, front or eddy and (2) those species (mainly fish) that seek shelter or food in seaweed, or lay their eggs on it, or whose surface-dwelling larvae are typically found among floating seaweed. The adults of the latter are usually independent of floating algae and show no particular adaptation to the rafting lifestyle.

These categories have been developed for fauna on floating macroalgae without or with tiny holdfasts (genera Ascophyllum, Fucus, Sargassum) and are not necessarily applicable to macroalgae with extensive holdfasts (e.g., genera Macrocystis, Durvillaea) harbouring a wide diversity of coastal organisms. Herein, we distinguish two major groups of rafters, namely the obligate rafters that depend at least during one stage of their life history on floating items and the facultative rafters that only occasionally occur on floating objects and can become passively dispersed by these.

Species from several phyla have assumed an obligate rafting lifestyle. Crustaceans, followed by gastropods, dominate in this group (see also Table 1). Not surprisingly most representatives from this group have been reported from the Sargasso Sea. Most species from the Sargassum fauna complete their entire life cycle on floating substrata. Stoner \& Greening (1984) mentioned several species that are considered to be endemic to the Sargassum community. Besides the grapsid crab Planes minutus they listed the amphipods Sunampithoe pelagica and Biancolina brassicacephala, the polycladid platyhelminth Hoploplana grubei, the nudibranch gastropod Scyllaea pelagica and the Sargassum fish Histrio histrio. Interestingly, most of these species maintain a limited motility: they can cling to a Sargassum patch but they can also let go and actively swim over short distances to adjacent patches.

The oceanic crabs from the genera Planes and Plagusia (Dellinger et al. 1997, Schubart et al. 2001, Donlan \& Nelson 2003) also possess the capability to move on and between floating items. Planes minutus can swim rapidly over short distances but has limited endurance. After about half an hour of swimming, limb movement slows down and the crabs start to sink (Davenport 1992). This author also mentioned that crabs rarely move $>5 \mathrm{~cm}$ away from their raft. The dependence on floating objects also becomes evident from the absence of this species in waters without flotsam. Other obligate rafters that retain high motility during their adult life are the isopod Idotea metallica and nudibranch gastropods. These species may also seek out new floating items when conditions on their original raft deteriorate.

Typical obligate rafters with a sessile lifestyle are most stalked barnacles. These feature internal fertilisation in the mantle cavity (e.g., Patel 1959), brooding of embryos during early development and pelagic larval stages of variable duration. Stalked barnacles achieve their entire life cycle in the pelagic environment and members of this group often dominate the rafting community, impressively demonstrating that successful reproduction and colonisation of floating items is possible even with inclusion of a pelagic larval stage.

Some pelagic species utilise floating items for reproductive purposes but are not dependent on them during the rest of their lives. The ocean-skaters from the genus Halobates attach their egg masses to floating objects (Stoner \& Humphris 1985, Andersen \& Cheng 2004). Similarly, the large harpacticoid copepod Parathalestris croni has a high affinity to floating Ascophyllum nodosum for reproductive purposes (Ingólfsson \& Ólafsson 1997). Naupliar larvae, unable to swim, hatch from eggs deposited by females on the plants. The larvae remain crawling on the alga until they metamorphose to the first copepodite stage, which is able to leave the algal substratum by swimming. 


\section{MARTIN THIEL \& LARS GUTOW}

Consequently, this species depends at least during part of its life cycle on a floating algal substratum that serves a nest function making Parathalestris croni a temporarily obligate rafter.

Temporary use of floating items has also been reported for many fish species, in particular for juvenile stages (Safran \& Omori 1990, Druce \& Kingsford 1995). Segawa et al. (1961) reported that the Pacific Saury Cololabis saira and flying fishes lay their eggs on floating seaweeds, which is underlined by observations from Dooley (1972) and Hobday (2000a) who mentioned that the flying fishes Cypselurus heterurus and C. californicus attach egg masses to floating macroalgae. Juvenile lumpsuckers Cyclopterus lumpus associate during their first year with diverse floating substrata where they find shelter and food (Davenport \& Rees 1993, Ingólfsson \& Kristjánsson 2002). Shaffer et al. (1995) described that the function of floating macroalgae change seasonally for associated juvenile splitnose rockfish Sebastes diploproa in coastal waters of Washington, U.S.. During spring and summer, floating macroalgae serve as spatial refuge for S. diploproa from vertebrate predators, while in autumn floating vegetation serves increasingly as food resource. Larger fishes associate with floating objects for protection from predators and to find food themselves (Gooding \& Magnuson 1967, Mitchell \& Hunter 1970, Moser et al. 1998, Castro et al. 2002, Nelson 2003). Kingsford (1995) suggested that floating algae increase the habitat complexity of the pelagic environment, thereby making them attractive for fish associates.

Most rafting organisms reported herein can be considered as facultative rafters that are only occasionally found on floating items. In the majority of cases, these species usually inhabit benthic substrata in the coastal zone. Rafting is generally viewed as a non-intentional excursion of these species onto floating items, which might then occasionally result in dispersal. Adults of facultative rafters need to be well adapted to survive both in benthic habitats and on floating items. For this reason it is not surprising that most facultative rafters are reported from floating items that are already densely colonised while still attached in coastal habitats. Many facultative rafters are reported from macroalgae that first develop in benthic environments where also initial colonisation proceeds. Upon detachment, these algae carry with them a diverse fauna of facultative rafters (Kingsford \& Choat 1985, Bushing 1994, Ingólfsson 1998, Hobday 2000a). Many facultative rafters also colonise floating items after these are detached (see, e.g., Ingólfsson 1995).

Interestingly, rafting has rarely been considered as an integral component in the life history of facultative rafters, even though some species appear well adapted to rafting (see above) and are found frequently and in large numbers on floating items. Some of these species may actively contribute to their substratum going afloat. For example, burrowing isopods and amphipods excavate holdfasts and stipes of common floating macroalgae (Edgar 1987, Chess 1993, Smith \& Simpson 1995, Thiel 2003a) and may thereby facilitate detachment and subsequent dispersal on these algae. Similarly root-boring isopods may cause breakage and subsequent floating of entire mangrove trees or parts thereof (Svavarsson et al. 2002). Since these peracarid borers feature direct development and local recruitment within the maternal patch, they may face a high risk of local inbreeding and it could be advantageous to increase dispersal frequencies by causing detachment of the positively buoyant burrowing substrata (Figure 11). Whether these or other species actively induce detachment (by strategic burrowing) and subsequent floating dispersal should be examined in future studies. It could be hypothesised that local demes that are detached and rafting on floating macroalgae contain a higher proportion of juveniles and subadults (i.e., individuals prone to colonise new microhabitats, see also Brooks \& Bell 2001a) than do demes in firmly attached plants.

Brooding in the past has primarily been viewed either as a consequence of morphological constraints (Strathmann \& Strathmann 1982) or as a mechanism to improve offspring survival (Ebert 1996, Chaparro et al. 2001). Since brooding in combination with local recruitment appears highly advantageous in the rafting environment, it cannot be completely discounted that in some facultative rafters, brooding has evolved as an adaptation to rafting dispersal. Rafting appears to 

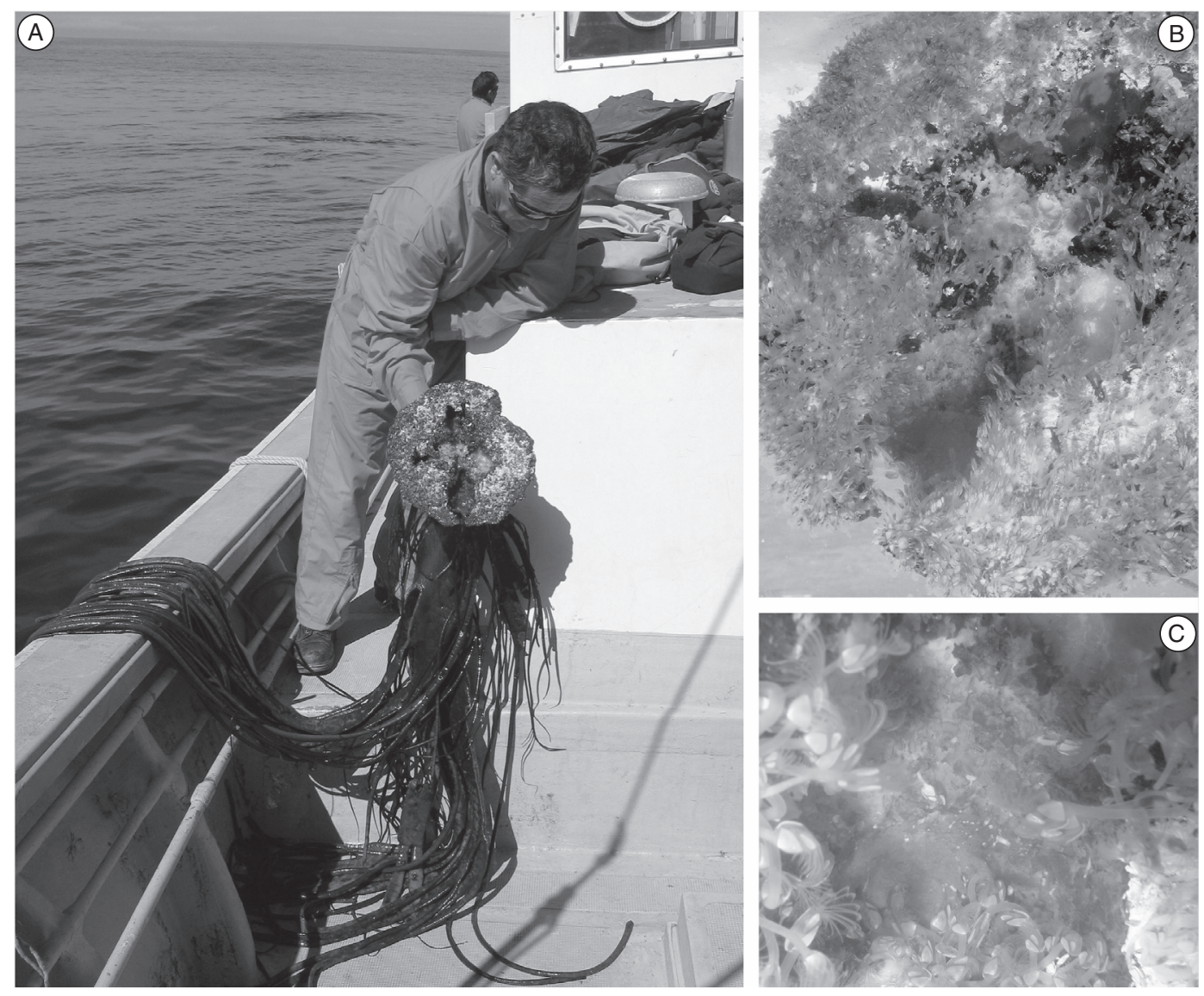

Figure 11 (A) Floating plant of Durvillaea antarctica colonised by dense assemblages of stalked barnacles; (B) Central part of the holdfast excavated by snails (Scurria scurra) and isopods (Limnoria chilensis); and (C) details of dense colony of L. chilensis.

have a significant effect on the geographic distribution, population dynamics and gene flow between local populations of some frequent rafters, and consequently it could be possible that natural selection has favoured the evolution of reproductive traits conducive to successful rafting (e.g., Ó Foighil 1989).

Besides obligate and facultative rafters, some authors also consider a third group of species, the 'accidental' rafters (Donlan \& Nelson 2003). We believe that this further subdivision is not useful since most of the facultative rafters go adrift accidentally, even though some species might actively contribute to detachment and subsequent dispersal of floating items.

\section{Interactions during rafting}

\section{Substratum-organism interaction}

Interactions between floating substrata and rafting fauna occur in both directions, i.e., substratum characteristics affect the colonisation by rafting organisms and rafters themselves have an influence on the durability of floating items. 


\section{MARTIN THIEL \& LARS GUTOW}

\section{Effect of substratum characteristics on rafters}

Floating substrata differ substantially in some characteristics important for survival and dispersal potential of rafters (Thiel \& Gutow 2004). Abiotic substrata do not provide food for rafters but typically have a relatively high longevity possibly permitting long-distance dispersal. Consequently, organisms that live on allochthonous food resources and that can attach to and survive on inert abiotic substrata may have good chances to become widely dispersed on these. In contrast, biotic substrata such as macroalgae and wood provide autochthonous food for rafters, and may thus be well suited for grazing and boring species. This is reflected in the high proportion of suspension feeders on abiotic substrata (plastics and pumice) and the common presence of grazing/boring species on biotic substrata (macroalgae and wood) (see also above, Figure 4).

Characteristics of floating items may thus act as a filter for organisms seeking attachment substratum. In many cases, unsuccessful colonisers will be weeded out when making first contact with an item, because substratum characteristics may simply prevent some organisms from settlement. For example, differences in the hardness of wood species have a selecting effect on organisms that can colonise these woods (Nair \& Saraswathy 1971, Borges et al. 2003). Floating algae may also produce substances that inhibit successful establishment of potential rafters (e.g., Sieburth \& Conover 1965, Wikström \& Pavia 2004). Calder (1995) found that some hydroid species occurred abundantly on Sargassum fluitans but not on S. natans and vice versa. Interestingly, this author mentioned that most hydroids collected on Sargassum were also found on wood, plastics and even tar balls. Conover \& Sieburth (1964) demonstrated that floating Sargassum plants show chemical antibacterial activity reducing the establishment of a bacterial biofilm, which is the first successional stage of overgrowth.

Substratum morphology and surface texture will also affect the rafting community. Simple surfaces (algal blades and plastic sheets) provide settlement substrata for sessile organisms such as spirorbid and serpulid polychaetes, bryozoans, hydrozoans and ascidians (Figure 12). This is reflected in the high proportion of these groups on plastic debris (Winston 1982, Ye \& Andrady 1991, Stevens et al. 1996, Winston et al. 1997, Barnes \& Sanderson 2000) and smooth surfaces such as nautiloid shells (Hamada 1964). Highly complex structures (algal holdfasts and tree roots) provide habitat for motile species such as errant polychaetes, ophiuroids and crustaceans. Thus, one might expect a high proportion of sessile rafters on smooth abiotic substrata and a high proportion of motile species on morphologically complex biotic substrata. Surface texture can also have an influence on successful establishment of potential colonisers (e.g., Bers \& Wahl 2004). Stevens et al. (1996) mentioned that "many plastics, including HDPE and PET, are hydrophobic and this may be a factor for rapid colonisation by Bryozoa as their settling larvae exhibit substratum specificity, generally avoiding wettable surfaces".

Post-settlement interactions between substratum and rafters may also influence which organisms may be able to persist on a floating item. For example, Wikström \& Pavia (2004) observed that young barnacles settled successfully on Fucus evanescens but then experienced high mortality. Isopods and barnacles that were only provided with tar balls as a substratum apparently did not suffer any short-term ill effects (Horn et al. 1970). However, barnacles growing on floating tar balls were on average smaller than animals from floating pumice indicating either that tar is slightly toxic to barnacles or that the softer tar is less suitable as attachment substratum than other floating items.

\section{Effect of substratum size on rafters}

Large floating items provide more space than do small objects. One consequence is that species found rafting on small objects are typically small. Carpenter \& Smith (1972) observed primarily diatoms and small hydroid colonies on small plastics (a few $\mathrm{mm}$ in diameter). In contrast, large 


\section{RAFTING OF BENTHIC MARINE ORGANISMS}

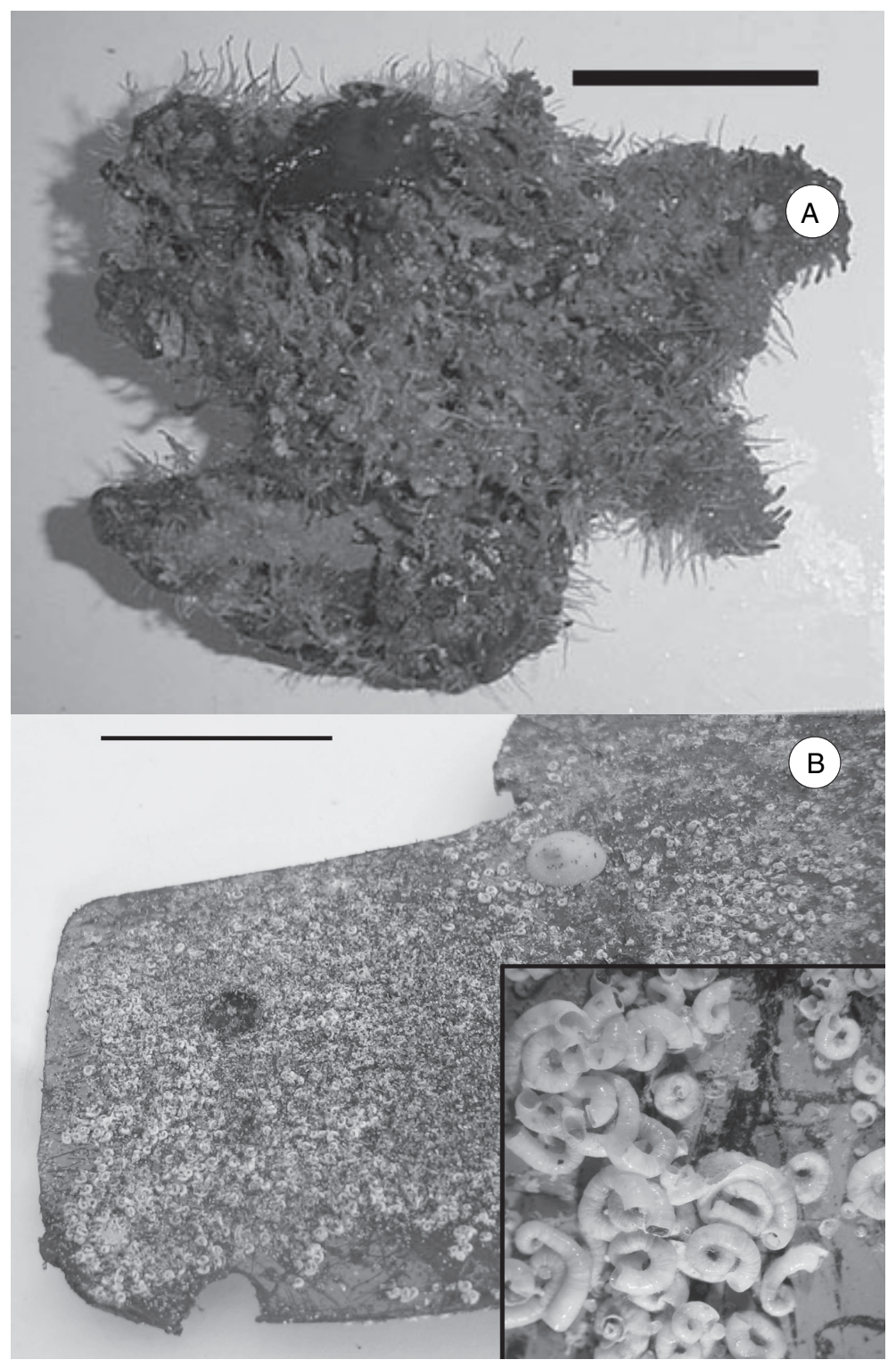

Figure 12 (A) Holdfast of floating Macrocystis integrifolia illustrating the complex structure of this substratum, which offers ample space for a wide diversity of sessile and mobile rafters. (B) Floating plastic showing simple surface structure offering primarily settlement substratum for sessile organisms (e.g., spirorbid polychaetes). Scale bar represents $5 \mathrm{~cm}$ in each case.

items or extensive rafts can support large rafters, including terrestrial vertebrates (e.g., Censky et al. 1998, see also references therein). The size of an item has also a strong influence on the density and species richness of the rafting community (see also Thiel \& Gutow 2004). Large items have a higher carrying capacity and can support a higher density of individuals. Various studies revealed a positive correlation between size or surface area of a floating item and the number of individuals or species of rafters (Figure 13), further supporting the fact that space on a raft may be limited and partitioned among rafters. 


\section{MARTIN THIEL \& LARS GUTOW}
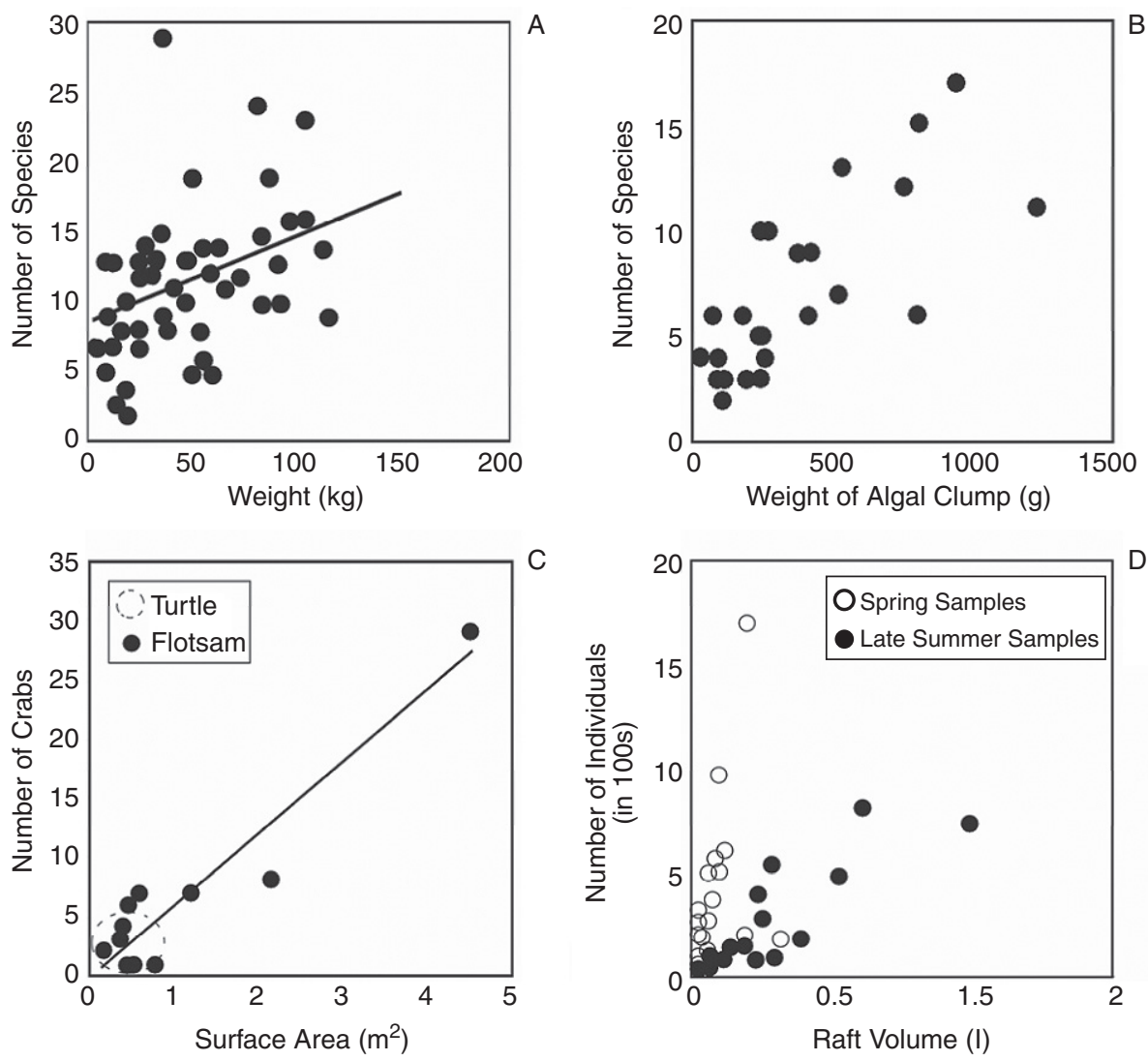

Figure 13 Relationship between the weight of algal clumps and the number of rafting species for (A) Macrocystis pyrifera and (B) Ascophyllum nodosum and Fucus vesiculosus; relationship between the size of a floating item and the number of rafting individuals for (C) crabs Planes minutes on flotsam, and (D) total rafters on floating Sargassum. Figures modified after Hobday 2000a (A), Ingólfssson 1995 (B), Dellinger et al. 1997 (C), and Fine 1970 (D).

For rafting organisms from floating macroalgae off Iceland, density was also positively correlated with the wet weight of the algal clumps, although in some species this relationship was not very distinct and temporally variable (Ingólfsson 2000). Higher densities of animals on larger clumps might result from a better protection from predators and a reduced risk of dropping off larger, more complex algal clumps. Furthermore, larger algal clumps are more likely to be encountered by actively swimming organisms and other floating algae (Ingólfsson 1995), further promoting colonisation. Ingólfsson (1998) showed that weight-dependent increase in species diversity was higher on large ( $\geq 62 \mathrm{~g}$ wet weight) algal clumps than on small ( $<62 \mathrm{~g}$ wet weight) clumps. He suggested that colonisation rate is significantly influenced by the enormous number of small fragments of macroalgae floating in the waters around Iceland, because these fragments harbour a considerable number of potential colonisers. The numbers of Gammarellus angulosus on algal clumps was significantly correlated with the weight of smaller algal fragments entangled in the clumps (Ingólfsson 2000). Finally, larger clumps simply provide more space, more food and more sites to cling to for a larger number of inhabitants, resulting in a positive correlation between the number of individuals and the weight of the clump (Ingólfsson 1998, 2000). 


\section{RAFTING OF BENTHIC MARINE ORGANISMS}

However, there is not always a positive relationship between substratum size and number of rafting individuals/species. Densities of tanaids and echinoderms on floating macroalgal mats at San Juan Island, Washington, were not correlated with algal wet weight (Highsmith 1985). The author suggested that different qualities of algae might be responsible for the observed lack of correlation. He observed that clean, bright-green samples harboured only very few animals while the oldest-appearing and most sediment-laden algal samples had the highest load of animals. He concluded that the clean and younger parts of the plants from the top of the canopy were less colonised by epibionts from the underlying benthos than were the old parts when detached from their substratum. This conclusion is supported by observations from Stoner \& Greening (1984) who found for floating Sargassum that patch age had a stronger effect on the abundance of rafters than patch size.

For floating Sargassum in the North Atlantic, Dooley (1972) found that seaweed biomass was positively correlated with the numbers of the two most abundant fish species (Histrio histrio and Stephanolepis hispidus), but no correlation was found between the total biomass of fishes and total algal biomass. Nelson (2003) found more fishes under large floating objects than under small floating objects (see also Moser et al. 1998).

In summary, the size of floating items has a strong influence on the number of rafters, even though other factors, in particular age of floating items, may affect this relationship. It is possible that older items, which have been afloat for longer time periods, are at a different stage of succession of the rafting community (see below).

\section{Substratum selection by potential rafters}

At present it is not entirely clear how selective potential rafters are at the moment of encountering floating items. Sessile organisms, at settlement, may select certain sites on a floating item. Hentschel (1922) mentioned that spirorbid polychaetes settle preferentially on pneumatocysts of floating Sargassum. Within-item selectivity has also been suggested by Ryland (1974) who discussed that larvae of the bryozoan Membranipora tuberculata and the spirorbid polychaete Janua formosa settle primarily on older parts of Sargassum plants. Besides these observations, little is known about which sites on a floating item are preferred by sessile rafters. In particular, it is unknown whether potential settlers can afford to reject a floating item upon encounter. However, several observations suggest that at least some common rafters are relatively unselective about their substratum. Calder (1993) suggested that hydroids settling on suspended wood panels typically are found on a variety of different substrata. Cornelius (1992a) emphasised that many hydroid species that are known to raft are substratum generalists. Also some bryozoan species are known from several types of floating items (see above). The fact that these and other sessile rafters occur on a wide variety of different substrata (e.g., Hentschel 1922, Winston et al. 1997) further indicates that they settle on any substratum, which may be due to the high unpredictability of subsequent encounters with other floating items.

Motile rafters appear to be more selective than non-motile ones with respect to their substratum. Floating items may offer different microhabitats to rafters (e.g., blades; stipes or holdfasts in macroalgae; trunk or root system in trees), and motile organisms may move towards the most suitable microhabitat within a floating item. These organisms may not only move within a floating item, but also between them. Exchange of motile organisms between rafts can occur when contacts among different floating items happen frequently. Indication that this indeed may occur comes from several studies on rafters from floating macroalgae. For example, Ingólfsson (1998) reported that certain species such as the herbivorous isopod Idotea granulosa and juvenile amphipods of the genus Gammarus colonised artificial mimics of algal fronds consisting of a black plastic material in lower numbers than genuine algal fronds. Numbers of harpacticoid species in algal clumps near 


\section{MARTIN THIEL \& LARS GUTOW}

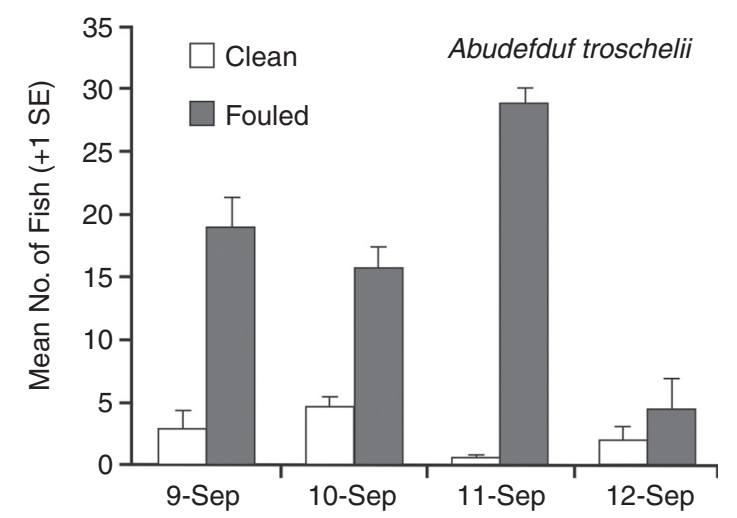

Figure 14 Numbers of the fish Abudefduf troschelii under artificial floats that were in clean state or colonised by a diverse fouling community. Figure modified after Nelson 2003.

Iceland were also found to depend on the algal species composition (Ólafsson et al. 2001). Pavia et al. (1999) remarked that epiphytic algae have a significant effect on the distribution of crustacean mesograzers inhabiting attached Ascophyllum nodosum. They suggested that the animals might benefit from increasing habitat complexity of fouled macroalgae providing better shelter from predators. Furthermore, epiphytes might be more palatable than the floating macroalgae for some mesograzers. The rafters Cyclopterus lumpus, Gammarellus angulosus and Dexamine thea apparently preferred Ascophyllum nodosum that was overgrown by the red alga Polysiphonia lanosa since their numbers were correlated positively with the weight of this epiphyte (Ingólfsson 2000). Most of these studies have been conducted in regions with high abundance of floating macroalgae, and consequently contact between different algal patches may commonly occur (see also Ingólfsson 2000), offering rafters the chance to select between items. Hobday (2000a) coined the term 'rafthoppers' referring to organisms that can switch between floating items.

The highest selectivity with respect to substratum characteristics can be expected for fishes, which are capable to move autonomously between floating items. This is demonstrated by the rapid colonisation of floating items by fish. Subsequent reshuffling of fish among floating items results within short time periods in a significant relationship between substratum size and fish number (e.g., Nelson 2003). Many fish associates appear rather selective about substratum size, concentrating under large floating items (e.g., Moser et al. 1998), but they also show a preference for items colonised by a diverse rafting community (Figure 14). Regardless of their preference criteria, many, and in particular large fish, show a relatively high selectivity for large, complex and already colonised rafts. The high degree of selectivity among these fish probably is a reflection of their high motility.

In summary, selectivity with respect to floating substrata appears to be a good reflection of the motility of rafting organisms, with sessile rafters being least selective and motile fish most selective (Figure 15). The limited degree of selectivity among sessile rafters may be an expression of limited encounter chances with floating substrata.

\section{Influence of rafters on floating substrata}

Rafting organisms can also exert a strong influence on floating substrata. For example, feeding by rafters on their floating substratum contributes to the continuous destruction of their raft. Proceeding disintegration of holdfasts of floating Macrocystis pyrifera from Tasmanian waters was mainly ascribed to the boring activity of isopods from the genus Phycolimnoria (Edgar 1987). Strong 


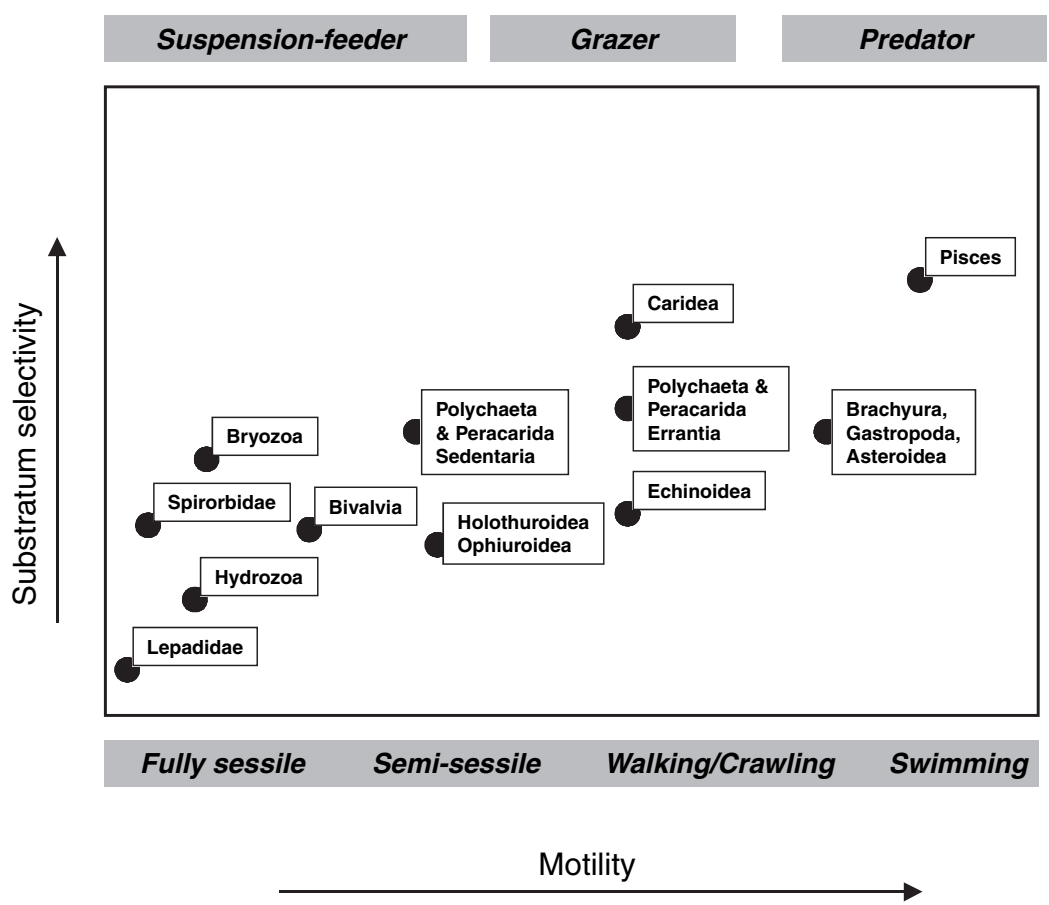

Figure 15 Schematic relationship between motility of principal rafting taxa and their substratum selectivity.

boring activity has also been observed for Limnoria stephenseni on attached holdfasts of Durvillaea antarctica at Macquarie Island (Smith \& Simpson 1995). As Stoner \& Greening (1984) mentioned that only few species of the Sargassum community directly consume their host plant, destruction of the algal raft might be a feature of facultative rafters originating from benthic habitats. Furthermore, floating Sargassum might be well protected from feeding by the active synthesis of toxic polyphenolic substances (Sieburth \& Jensen 1969).

For floating Macrocystis pyrifera in Californian waters, decomposition of algal fronds was strongly temperature dependent (Hobday 2000b). While at temperatures below $20^{\circ} \mathrm{C}$ the decomposition rate was quite low, a dramatic increase in the aging rate of the algae (measured as loss of algal blade length) was observed at higher temperatures. The relationship between longevity of floating algae and water temperature can also be seen from the good condition of algal fronds floating in cold Icelandic waters. Even after about $6 \mathrm{wk}$ of floating, plants of Ascophyllum nodosum hardly exhibited any sign of decomposition (Ingólfsson 1998). In contrast, plants of this species incidentally found near the equator did not appear 'particular robust' (John 1974). However, since John (1974) did not mention any grazer associated with the plants it is not known whether this lack of robustness is a result of grazing or of temperatures far above the physiological optimum of this rather northern species. Boring of associated rafters is also responsible for decomposition of floating wood (Emery 1963), which in the case of large trees, though, is more resistant to destruction than macroalgae and may persist sufficiently long to carry rafters over distances $>1000 \mathrm{~km}$.

Another important effect, which rafting organisms have on their substratum, is that they contribute to an increase in weight of a floating item. Hentschel (1922) and Parr (1939) suggested that the weight addition of calcareous skeletons might contribute to the sinking of macroalgae (Sargassum). In a personal communication to Johnson \& Richardson (1977) N.J. Blake reported on small fragments of Sargassum in the Gulf of Mexico that were so heavily encrusted by Membranipora sp. and Lepas sp. that it was only barely buoyant. Zaitsev (1970) measured the 


\section{MARTIN THIEL \& LARS GUTOW}

specific density of floating Sargassum without epibionts to range from $0.785-0.788 \mathrm{~g} \mathrm{ml}^{-1}$. Under natural conditions, Woodcock (1950) found specific densities varying between 0.905 and $0.955 \mathrm{~g} \mathrm{ml}^{-1}$. Consequently, with a proportion of $13-18 \%$, epibionts contribute significantly to the overall specific density of the floating assemblage. Loss of buoyancy as result of heavy fouling coverage has also been observed for plastics (Holmström 1975, Ye \& Andrady 1991, Winston et al. 1997, Moore et al. 2001). Dixon et al. (1981) described that plants of Macrocystis pyrifera fouled by Membranipora sp. suffered greater blade loss than clean plants probably because fouled blades are fragile and break off easily and because fish bite off chunks of blade while foraging on the attached bryozoans. Similar observations were made by Scheibling et al. (1999).

Epibionts growing on algae may also positively affect the assimilation potential of their substratum as had already been suspected by Hentschel (1922) for Sargassum. The existence of a positive relationship between rafting epibionts and floating macroalgae is supported by results from studies by Pavia et al. (1999) who demonstrated that benthic macroalgae benefit from the presence of grazers when the latter reduce the overgrowth on the algal substratum by preferably feeding on epiphytic algae. Whether epiphytic algae affect photosynthesis by shading parts of the host plant or whether the two plant species compete for nutrients from the surrounding waters is not clear. Metabolism of the plant can also be affected by encrusting epibionts such as bryozoans. However, where the latter shade parts of the plant, concentrations of chlorophyll and other accessory pigments may increase, maintaining photosynthetic efficiency at high levels (Muñoz et al. 1991). Photosynthesis is further favoured by $\mathrm{CO}_{2}$ released during respiration of encrusting bryozoans serving as a source of photosynthetic inorganic carbon (Muñoz et al. 1991). While on the one hand attached bryozoans limit nitrogen uptake of the plant, excretions from the animals function, on the other hand, as an important source of inorganic nitrogen for the alga (Hurd et al. 1994). For example, fishes aggregating underneath floating objects may contribute to an enrichment of the surrounding water with nutrients (Deudero \& Morales-Nin 2001a). Culliney (1970) also observed phosphorus concentrations in water surrounding patches of floating Sargassum to be substantially higher than in the otherwise oligotrophic Sargasso Sea, possibly enhancing growth of the algae. For floating plastics Winston et al. (1997) suggested another positive effect of rafting organisms on their rafting substratum. They discussed that rafters covering plastic items might protect the substratum from the direct impact of sun radiation, which is known to enhance plastic degradation.

In summary, substratum characteristics can affect the abundance and species composition of the rafting community, while rafters themselves affect the longevity (and growth) of floating items at the sea surface.

\section{Interactions within the rafting community}

Besides interactions with floating substrata, there exist many ecological interactions among rafting organisms themselves. These interactions are similar to those known from other environments. The two principal resources utilised by most rafting organisms are space and food/nutrients. Space availability depends on the size and specific surface area of the floating object. On a given raft, space is not extensible unless some of the rafting organisms themselves add new colonisable space either by excavating into floating items or by constructing new surfaces. Due to the limiting character of this resource, strong competition for space can be expected on most floating items. The second resource, food supply, is at least partly supplied from the surrounding environment. Many suspension feeders, detritus feeders, predators and scavengers live on allochthonous food resources, which may be continuously replenished from the surrounding water body. Similarly, nutrients for rafting

plants may be supplied by rafting fauna during the journey of floating items (Stoner \& Greening 1984). The high proportion of suspension feeders among the rafting fauna underlines that trophic 


\section{RAFTING OF BENTHIC MARINE ORGANISMS}

interactions between the rafting community and the surrounding water body are important. However, the fact that grazers and predators/scavengers are abundant on rafts also suggests that interactions within the rafting community play an important role. Most of these latter organisms will consume other rafters, but some rafters will also have positive effects on the establishment or persistence of others.

\section{Facilitative interactions}

It is well known that some species only colonise substrata after other organisms have conditioned these. For example, teredinid bivalves preferentially colonise wood that has been preconditioned by fungi (Nair \& Saraswathy 1971). Also, wood-boring isopods from the genus Limnoria only attack wood that is already colonised by wood-degrading fungi and bacteria (Daniel et al. 1991). Similarly, settlement of sessile organisms on plastic surfaces is enhanced if these are covered with biofilms (Lam et al. 2003).

Rafting organisms themselves can contribute to the structural complexity of floating substrata. Boring species construct burrows and crevices that can subsequently be used by other organisms as hiding places. For example, El-Shanshoury et al. (1994) found bacteria and fungi in isopod burrows from wood. Also, Kohlmeyer (1984) remarked that the fungi Halosphaeria quadricornuta and $H$. salina occurred in teredinid and limnorid burrows, and it is probable that many other organisms find refuge in these burrows. Svavarsson (1982) found the isopod Caecijaera borealis in the burrows of Limnoria borealis. Similarly, Smith \& Simpson (1995) noted that most motile fauna in benthic holdfasts of Durvillaea antarctica is found in cavities created by the boring isopod Limnoria stephenseni. Nair \& Saraswathy (1971) reported a diverse fauna including polychaetes, turbellarians and gastropods (among others) from burrows made by shipworms.

Species with an erect growth form also contribute to the structural complexity of floating substrata. For example, many authors remarked that crabs from the genus Planes find shelter among dense colonies of stalked barnacles (e.g., Jara \& Jaramillo 1979). Caprellids and other crustaceans with good clinging abilities find good substratum support on erect bryozoans and hydrozoans, and consequently it is not surprising that these crustaceans only appear in large numbers after a floating item has been colonised by these sessile organisms (e.g., Tsikhon-Lukanina et al. 2001a). Large numbers of caprellids have been reported on floating buoys heavily overgrown with hydrozoans and bryozoans (Thiel et al. 2003).

\section{Competitive interactions}

One of the first accounts of competitive interactions among organisms living on floating items was provided by Hentschel (1922), who remarked for the Sargassum community that "competition for space and mutual displacement definitely occurs". He inferred intraspecific competition in dense assemblages of spirorbid polychaetes, which may hamper each other. Interspecific competition was also described by Hentschel (1922) who observed that hydrozoans overgrow bryozoans, which themselves may overgrow spirorbid polychaetes. Finally, diatom films may cover all these sessile organisms that are commonly growing on the blades of Sargassum. Individual patches of Sargassum often are completely dominated by one hydrozoan species, while other patches may harbour a different hydrozoan species (Hentschel 1922). Similar observations had been made by Parr (1939), who observed that those hydroid species, which he classified as dominants, hardly ever occurred together on the same Sargassum plant. These monospecific hydroid assemblages may be the result of competitive exclusion: Butler et al. (1983) mentioned that "branched and erect larger hydroids ... overgrow the simpler hydroids and either smother or outcompete them". Bryozoans from plastic items were able to overgrow most other sessile rafters (Stevens et al. 1996, Winston et al. 1997). 


\section{MARTIN THIEL \& LARS GUTOW}

Some bryozoan species found on floating items were better competitors than others, with Celleporina hemiperistomata being the superior competitor for space (Stevens et al. 1996). Competition for space among rafting organisms has also been suggested by Tsikhon-Lukanina et al. (2001a).

Rafting organisms also compete for food, regardless of whether they feed on autochthonous or allochthonous food resources. Most sessile epifauna from Sargassum feed on planktonic organisms, primarily microalgae (Hentschel 1922). Tsikhon-Lukanina et al. (2001a) remarked that bryozoans and juvenile stages of lepadid barnacles, both of which feed on fine suspended matter, compete for food resources. When growing, barnacles may shade bryozoans and limit access of the latter to suspended food - the competitive advantage of stalked barnacles over bryozoans on floating items apparently is reflected in higher rates of biomass increase in the former compared with the latter (Tsikhon-Lukanina et al. 2001a). Stalked barnacles do not seem to have negative effects on the motile fauna living on floating items as observed by Tsikhon-Lukanina et al. (2001a). These authors also emphasised the close overlap in food spectra of rafting barnacles, isopods and decapods. They suggested that competition among these groups is mitigated by the fact that they form local 'microcolonies' that are spatially separated (Tsikhon-Lukanina et al. 2001a). A close overlap in food spectra between rafting species was also observed by Sano et al. (2003), further supporting the conclusion that some rafters compete for food resources.

Davenport \& Rees (1993) expected the dominant herbivores on floating seaweeds in the Irish Sea, isopods of the genus Idotea and the amphipod Gammarus locusta, to compete for food on their algal raft. Strong competition between ecologically similar Idotea species on floating macroalgae was also hypothesised by Tully \& Ó Céidigh (1986a). These suspicions were confirmed through experimental studies by Franke \& Janke (1998) who revealed strong interspecific and intraspecific competition in the two species I. emarginata and I. baltica. They frequently observed cannibalism, and suggested that I. baltica utilises floating macroalgae as habitat in order to escape the competitively superior I. emarginata. Gutow \& Franke (2003) interpreted the disappearance of I. metallica in mixed cultures with I. baltica also as a result of interference competition.

Cannibalism has also been reported by Adams (1960) for juveniles of the Sargassum-fish Histrio histrio. Another example for intraspecific competition was given by Ingólfsson (2000) who found in one bight of Iceland a surprising negative correlation between the number of juvenile Cyclopterus lumpus and the weight of floating algal patches. He suggested territorialism and the possibility of cannibalism within the species as possible explanations for the clumped distribution of this species.

\section{Consumer interactions}

Plant-herbivore interactions may occur between rafting grazers and floating algae (see above) or between these grazers and rafting algae growing on floating substrata. Carpenter (1972) suggested that the blue-green epiphyte Dichothrix fucicola serves as a valuable food source for herbivorous organisms on floating Sargassum in the North Atlantic. Edgar (1987) attributed the disappearance of small filamentous algae from experimentally tethered holdfasts of Macrocystis pyrifera to invertebrate grazing. Many rafters from floating Sargassum off Japan feed on floating algae and their epiphytes (Sano et al. 2003). Gutow (2003a) revealed that Idotea metallica is sustained by fouling organisms on abiotic floating substrata, while this food resource can hardly satisfy the requirements of the congener I. baltica.

Predator-prey interactions are very common within the rafting community itself or between rafting organisms and associated species (fishes, turtles and birds). Edgar (1987) suggested that predation pressure of holdfast-dwelling organisms keeps the surface of the holdfasts clear of sessile organisms. He observed that abundances of bivalves appeared to be higher on initially defaunated holdfasts than on untreated ones. Some of the obligate motile rafters prey on sessile organisms. 


\section{RAFTING OF BENTHIC MARINE ORGANISMS}

For example, rafting crabs and isopods feed on a variety of items, including rafting fauna (TsikhonLukanina et al. 1986, Sano et al. 2003). Many of the rafting nudibranchs prey on sessile hydroids; Il'in \& Roginskaya (1987) also reported that the nudibranch Fiona pinnata preys intensively on stalked barnacles.

Many organisms that associate temporarily with floating items feed intensively on rafting fauna and flora. Kirkman \& Kendrick (1997) noted that bridled terns (Sterna anaethetus) along the Western Australian coast feed exclusively on organisms associated with floating Sargassum spp. accumulated in front of local beaches. Similarly, juvenile loggerhead turtles gathered in convergence zones with high concentrations of Sargassum spp. and other floating items (plastics and tar balls), where they feed on the abundant rafting organisms and floating substrata (Witherington 2002). Turtles may be passively transported to these convergence zones where they remain and feed on associated biota for long time periods. Small turtles themselves may become prey of large fish or birds (Witherington 2002). Stomach content analyses of fishes caught from floating Sargassum in the Sargasso Sea reflected the rafting community quite well (Dooley 1972). Many of the fishes associated with floating Sargassum off Japan feed intensively on the rafting community, including isopods, amphipods and decapod crustaceans (Ida et al. 1967). Pieces of Sargassum sp. itself and other floating objects as well as crabs and shrimps that are known from the Sargassum community were found in the stomachs of large tropical and subtropical pelagic fishes such as dolphin, Coryphaena hippurus, and tuna, Thunnus sp., indicating that these species feed on all parts of rafting assemblages (Manooch \& Hogarth 1983). Winston et al. (1997) observed abundant scrape marks on floating plastics and inferred that fish also prey on the rafting community on plastics. Fishes may also feed on eggs deposited on floating items (Kimura et al. 1958). Butler (1975) and Horn et al. (1970) reported on tar balls found in the stomach of the saury (Scomberesox saurus), which might have been ingested accidentally by the fish while foraging for associated crustaceans.

In particular, many juvenile fishes associated with floating items prey intensively on rafting organisms (Safran \& Omori 1990, Shaffer et al. 1995). Postlarval and juvenile lumpsuckers Cyclopterus lumpus appear to be opportunistic predators specialised on weed patches in the Northeast Atlantic (Davenport \& Rees 1993, Ingólfsson \& Kristjánsson 2002). The animals attach to the seaweed fronds and feed on crustaceans and fish larvae concentrated beneath the weed cover (Figure 16). Ingólfsson \& Kristjánsson (2002) reported ontogenetic changes in diet composition, with very young individuals feeding predominantly on crustacean larvae and small arthropods, while larger juveniles increasingly feed on adult crustaceans, including amphipods and isopods. Daborn \& Gregory (1983) also found the amphipod Calliopius laeviusculus, a common rafter on floating seaweeds, to be an important food item for juvenile lumpsuckers. Even insects entangled in floating algae in the Irish Sea have been found in the stomach of juvenile Cyclopterus lumpus (Davenport \& Rees 1993). Ontogenetic changes also were observed in the splitnose rockfish Sebastes diploproa, which associates with floating macroalgae during early life (Shaffer et al. 1995).

Anraku \& Azeta (1967) remarked that yellowtails Serolia quinqueradiata, associated with floating macroalgae, fed primarily on non-rafting organisms - caprellid amphipods were the only rafting fauna that could clearly be identified as such. Floating items often are accumulated in convergence zones, where planktonic food is also concentrated (Shanks 2002), and fish associated with floating items may benefit from these concentrations of plankton. However, Deudero \& Morales-Nin (2001b) observed a mismatch between the abundance of potential prey and predatory fishes, indicating that fish-aggregating devices represent fish nursery areas, providing spatial refuge from predators rather than feeding grounds.

Consumer interactions may also vary among items. Winston (1982) suggested that predation pressure around floating plastics is much lower than around floating macroalgae. This may be due to the lower biomass of the rafting community on abiotic floating items (plastics) compared with 


\section{MARTIN THIEL \& LARS GUTOW}

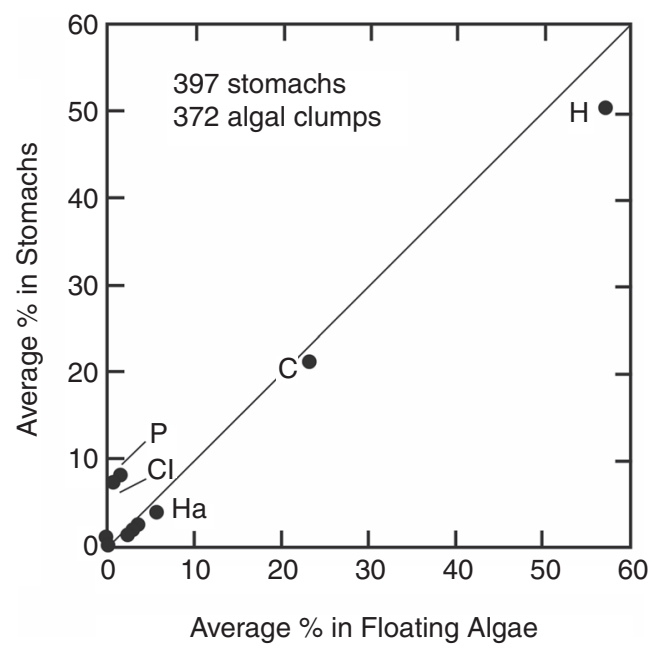

Figure 16 Relationship between frequency of potential prey organisms on floating algal patches and in fish stomachs of Cyclopterus lumpus sampled with these patches. Figure modified after Ingólfsson \& Kristjánsson 2002.

that on biotic items (macroalgae). In contrast, Brooks \& Bell (2001b) assumed that foraging success of predators around detached macroalgae might be suppressed (compared with the water column) due to the structural complexity of the algae. While the exact influence of substratum characteristics on predation rates may require research attention in the future, in general it can be summarised that predation pressure around floating items is high.

\section{The rafting food web}

As shown above, the rafting community includes primary producers and various levels of consumers. Zaitsev (1970) provided a schematic view of the ecologic relationships in the surface layer of the open ocean. He emphasised the fact that dead organic matter is imported into the system, and that a variety of predators contribute to export of living organic matter (Figure 17A). Cheng (1975) also noted the importance of predator-prey pathways in her scheme of pleustonic food-chain relationships (Figure 17B). Stomach analysis of common rafters and of fishes associated with floating items supported these insights by confirming that many rafters feed on detritus and microorganisms supplied to the raft, and that fishes associated with rafts feed both on rafters as well as on smaller fish. Sano et al. (2003) found that some rafters consume phytoplankton and small detritus while others have a more carnivorous diet, feeding mainly on crustaceans (Figure 17D). Besides these, there are many rafters that have a less catholic diet, being characterised mainly as omnivores. The analysis of Sargassum macrofauna by Butler et al. (1983) and Stoner \& Greening (1984) also revealed the high proportion of omnivorous rafters, feeding on detritus, sessile and motile fauna (Figure 17C). This dominance of opportunistic secondary consumers among the rafting community is not surprising since floating items gather in convergence zones where also particulate organic matter accumulates. Morris et al. (1976) remarked that "the limits of food availability within the Sargassum community have imposed a necessity for the largest organisms (crabs, shrimps, and fish) to have the greatest number of alternative food sources".

Several authors also emphasised the fact that excretions of rafting fauna may benefit either floating macroalgae themselves or epiphytic algae (Culliney 1970, Deudero \& Morales-Nin 2001a). Similar to that reported for benthic habitats (Taylor \& Rees 1998), these dissolved nutrients probably contribute to enhanced growth and, thus, to the longevity of floating or rafting macroalgae and 

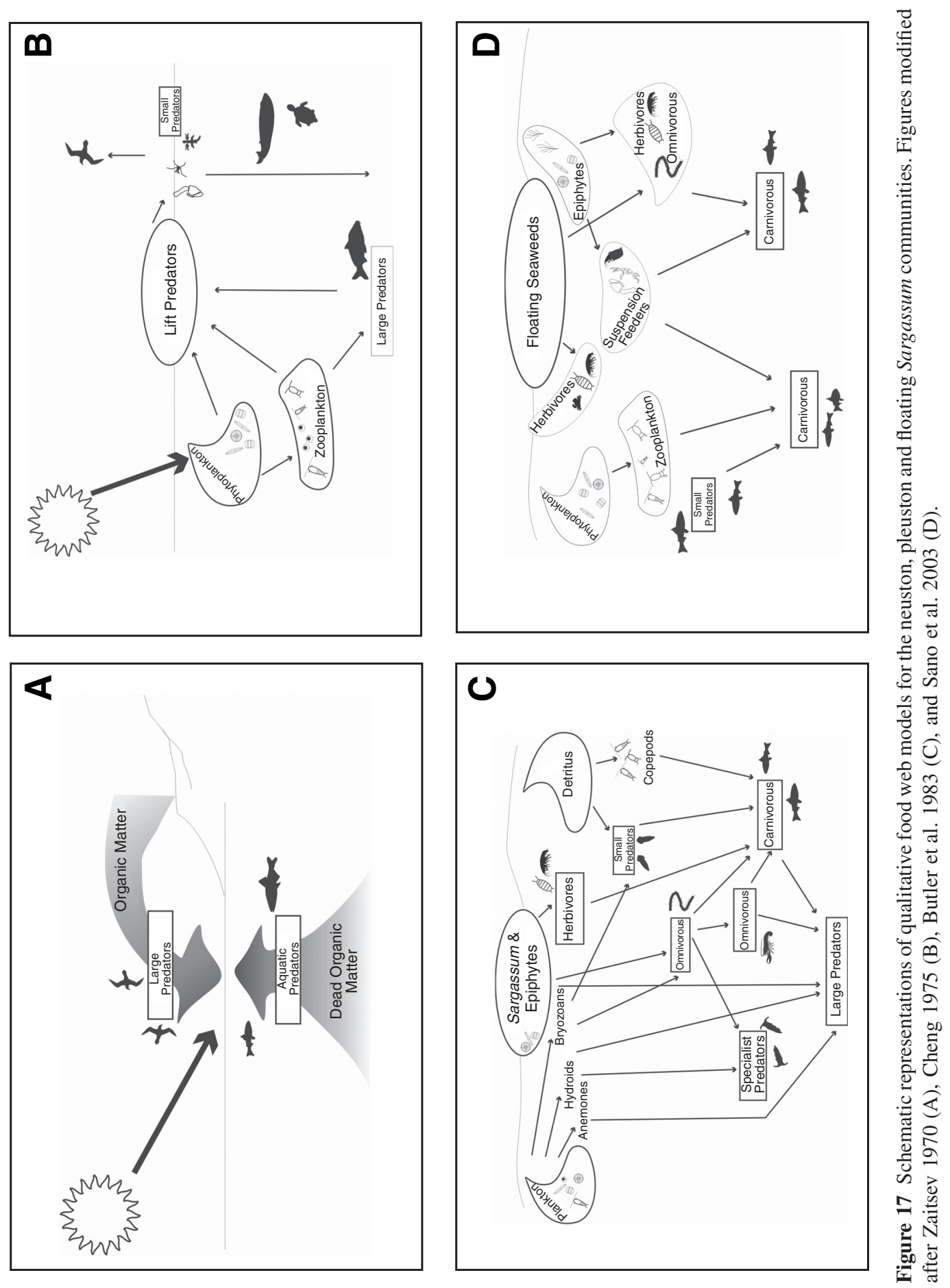


\section{MARTIN THIEL \& LARS GUTOW}

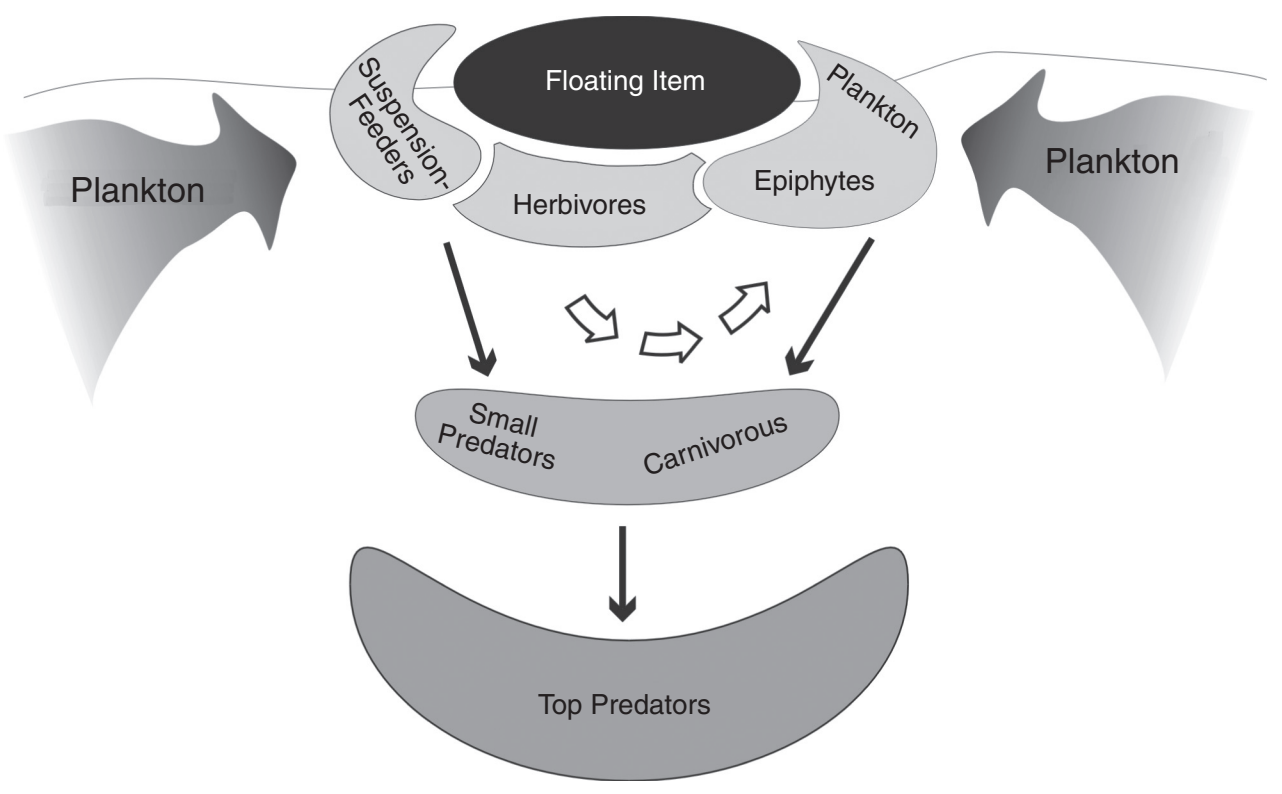

Figure 18 Simplified food web model for rafting community.

attached micro-organisms. Consequently, primary production may be higher on and around floating items than in the surrounding water bodies. Smith et al. (1973) determined the respiration of the Sargassum community to be $2-5$ times greater than the surface layer $(1-2 \mathrm{~m})$ plankton respiration.

In summary, trophic processes are enhanced on floating substrata compared with the surrounding surface waters. The high import of dead organic matter (plant detritus and animal carcasses) is converted by the rafting community into living organic matter, partly sustaining the rafting community itself and being exported to the pelagic environment via associated fish predators (Figure 18). Thus, floating items appear to have a catalytic function at the sea surface, similar as has been reported for mussel beds and coral reefs in benthic habitats (Prins et al. 1998, Wild et al. 2004) or for particulate organic matter (POM) in the water column (Azam \& Long 2001).

\section{Temporal and spatial variability of the rafting community}

\section{Estimates of floating duration}

As a result of the observed ecological interactions and of growth of rafters, it can be expected that the rafting community experiences substantial changes during the journey. However, in order to reveal the pattern of the succession it is important to have reliable estimates of the floating duration, which is not a trivial task. Several approaches have been taken. Strong indication for temporal changes comes from experimental studies in which entire plants or parts of floating macroalgae were tethered in coastal habitats (reviewed in Thiel 2003c). Even though in these cases floating duration is exactly known, these studies suffer from the drawback that they are conducted in the vicinity of coastal source populations and the observed succession may not necessarily be representative of the open ocean situation. Other authors collected floating items at various distances from a potential source region, where distance may be used as an estimate of floating duration. Using this approach, Ingólfsson (1995) revealed a strong decrease in abundance of common rafting organisms with increasing distance from the shore. Also, Donlan \& Nelson (2003) found that species richness on floating items (mainly wood) varied significantly with distance from the coast, 


\section{RAFTING OF BENTHIC MARINE ORGANISMS}

but they noted that this relationship was weak. This may be due to the fact that distance from the next coast is not a good estimator for floating duration (e.g., Hobday 2000a), since the underlying assumption, namely that transport direction of floating items is away from the shore, may not be fulfilled. This is not necessarily the case since oceanic currents or wind direction may be along-shore in their direction or even push items towards the shore (for overview see Thiel \& Gutow 2004). In some cases, time and place of introduction of a floating item is well known (e.g., anthropogenic items or volcanic pumice) and thus reliable estimates of floating duration can be obtained.

Another method to estimate rafting duration is based on the size of particular rafters or the integrity of rafting substrata (e.g., Stoner \& Greening 1984, Hobday 2000b, Tsikhon-Lukanina et al. 2001a). This approach is based on the same principle used by forensic scientists (e.g., Sorg et al. 1997), namely to utilise approximate growth rates of common indicator organisms/rafters (e.g., stalked barnacles) to reach minimum estimates of rafting duration. Assuming that stalked barnacles colonise items soon after these went afloat and combining this with maximum growth rates (see Table 17), one can obtain relatively reasonable estimates of floating duration. Many authors have used this principle and sizes of some rafters in order to make inferences about the minimum duration of floating (e.g., Hamada 1964, Horn et al. 1970, Jokiel 1984, 1990a, DeVantier 1992, Tsikhon-Lukanina et al. 2001a). Helmuth et al. (1994) used percentages of branch points of floating Macrocystis pyrifera plants colonised by stalked barnacles to estimate floating time. In addition to this measure, these authors also utilised blade length, a measure that has been further refined by Hobday (2000b). Ryland (1974) and Niermann (1986) used Sargassum colour and condition to obtain approximate age estimates. Stoner \& Greening (1984) had used a combination of these last measures to categorise floating macroalgae in young, medium and old patches. Utilising all these different approaches to obtain estimates of the floating duration of common substrata and their rafting organisms, some consistent patterns of the temporal succession of the rafting community emerge.

\section{Successional changes of rafting community}

\section{Species exchanges}

During the rafting journey, organisms frequently abandon, colonise, thrive or are suppressed on floating items. Depending on their biological characteristics, some species are capable of quickly colonising newly available floating objects, while the same species may not be able to withstand increasing competition or predation pressure on their rafts. Other organisms are poor colonisers, but once settled on a floating item they may be able to establish dense populations, excluding other organisms.

Some floating substrata begin their journey with a diverse fauna, while others start in a clean state (most plastics and volcanic pumice). Macroalgae and mangrove trees that are detached from benthic habitats, carry with them many of their original inhabitants. Edgar \& Burton (2000) observed that many invertebrates abandoned floating Durvillaea antarctica rapidly after detachment of the alga. However, the few species that were still present on the plants after one day of floating have a good chance to survive for longer periods adrift. Loss of original inhabitants from recently detached macroalgae was also reported by Edgar (1987), Kingsford \& Choat (1985), and Vásquez (1993). Caprellid amphipods, for example, have been seen to descend back to the sea bed if detached Sargassum plants start to float to the sea surface (Takeuchi \& Sawamoto 1998). Three main factors are responsible for this rapid loss of original inhabitants (Thiel 2003c): (a) they actively abandon floating items, (b) they cannot hold onto floating items, (c) they cannot resist the high predation pressure in the pelagic environment. It is thus not surprising that some of the most common organisms lost immediately after detachment are echinoderms, brachyuran crabs and large gastropods 


\section{MARTIN THIEL \& LARS GUTOW}

(Kingsford \& Choat 1985). Floating clumps of Sargassum muticum collected at only $200 \mathrm{~m}$ offshore also carried less species and substantially lower numbers of rafting organisms than similar plants that were still attached (Norton \& Benson 1983).

Even though many species are lost shortly after detachment, new species immediately start to colonise the floating macroalgae as revealed by the presence of some species not commonly reported from attached macroalgae. Many of these new colonisers come from coastal habitats or from the pelagic environment through which floating items pass during this initial phase of travel. The species turnover occurring shortly after detachment of floating macroalgae or after appearance of new floating items can be nicely visualised by the four categories of colonising organisms described by Ingólfsson (1995) for macroalgae floating off the coast of Iceland. Organisms from the first group typically live in these algae when growing attached in the intertidal zone, while animals from the three other groups colonise floating algae after detachment. Not surprisingly, the first group of the original algal inhabitants is the most diverse, comprising typical members from the intertidal algal belt of temperate North Atlantic shores (amphipods, isopods, polychaetes, gastropods, midges, bivalves, oligochaetes and bryozoans). Members of these groups experience substantial losses between coastal and offshore waters and many of them lack completely in floating algae collected $>60 \mathrm{~km}$ from the nearest shore (Ingólfsson 1995). The second group comprises mainly insects and some oligochaetes that colonise macroalgae cast up on the shore. These organisms might be carried out to the sea on macroalgae resuspended during high tide. However, it is questionable if subsequent rafting of these supratidal animals is efficient, since (1) they may not be well adapted to clinging to floating substrata and (2) temporary stranding most likely damaged the plants, thereby severely limiting their floating capacity. The species of the third group identified by Ingólfsson (1995) normally live in subtidal benthic habitats and also colonise the seaweeds after these are detached and afloat. Members of this group are gammarid amphipods and (less typical) idoteid isopods. The fourth group consists of planktonic or neustonic species such as the lumpsucker Cyclopterus lumpus and the harpacticoid copepod Parathalestris croni that also colonise the algae when they are already afloat. The amphipod Gammarus wilkitzki probably also belongs to this group. While floating items of biotic origin primarily harbour original inhabitants, shore and subtidal organisms, items of abiotic origin are relatively unattractive to these organisms and are primarily colonised by pelagic organisms (Figure 19).

The rapid colonisation of floating macroalgae in coastal waters probably is a consequence of the high motility of many coastal invertebrates (Ingólfsson 1998). Other studies revealed a very high turnover of individuals and species in benthic macroalgae (Virnstein \& Curran 1986, Smith 1994, Taylor 1998). In particular, amphipods rapidly colonise moving macroalgae while these travel through coastal habitats (Brooks \& Bell 2001b). Some species that can float autonomously or swim actively for short time periods may also cling to floating items when coming in contact with them (Highsmith 1985). The capability of species to move from and onto moving substrata results in substantial changes of the rafting community while still floating in the vicinity of benthic coastal habitats. Based on the relationship between raft age and size of common rafters, Hobday (2000a) suggested that exchange between rafts does not occur very frequently. He remarked, though, that some ubiquitous rafters, even though not being very good swimmers, can swim back to floating items when separated from them. Species not well-adapted to raft on floating items may abandon these and return to benthic habitats, while others colonise an item either when coming in direct contact with it (supratidal organisms) or by actively swimming towards it (subtidal and pelagic organisms).

As outlined in the previous paragraph, colonisation of floating items in coastal habitats can occur via adult stages (Kingsford \& Choat 1985, Brooks \& Bell 2001b) but also via settling larvae (e.g., Ingólfsson 1998). After leaving the nearshore waters, floating items accumulate in drift rows in convergence zones where also larvae of many organisms are concentrated (Kingsford \& Choat 1986, Jokiel 1990a, Shanks 2002). This may facilitate colonisation of floating objects by organisms 

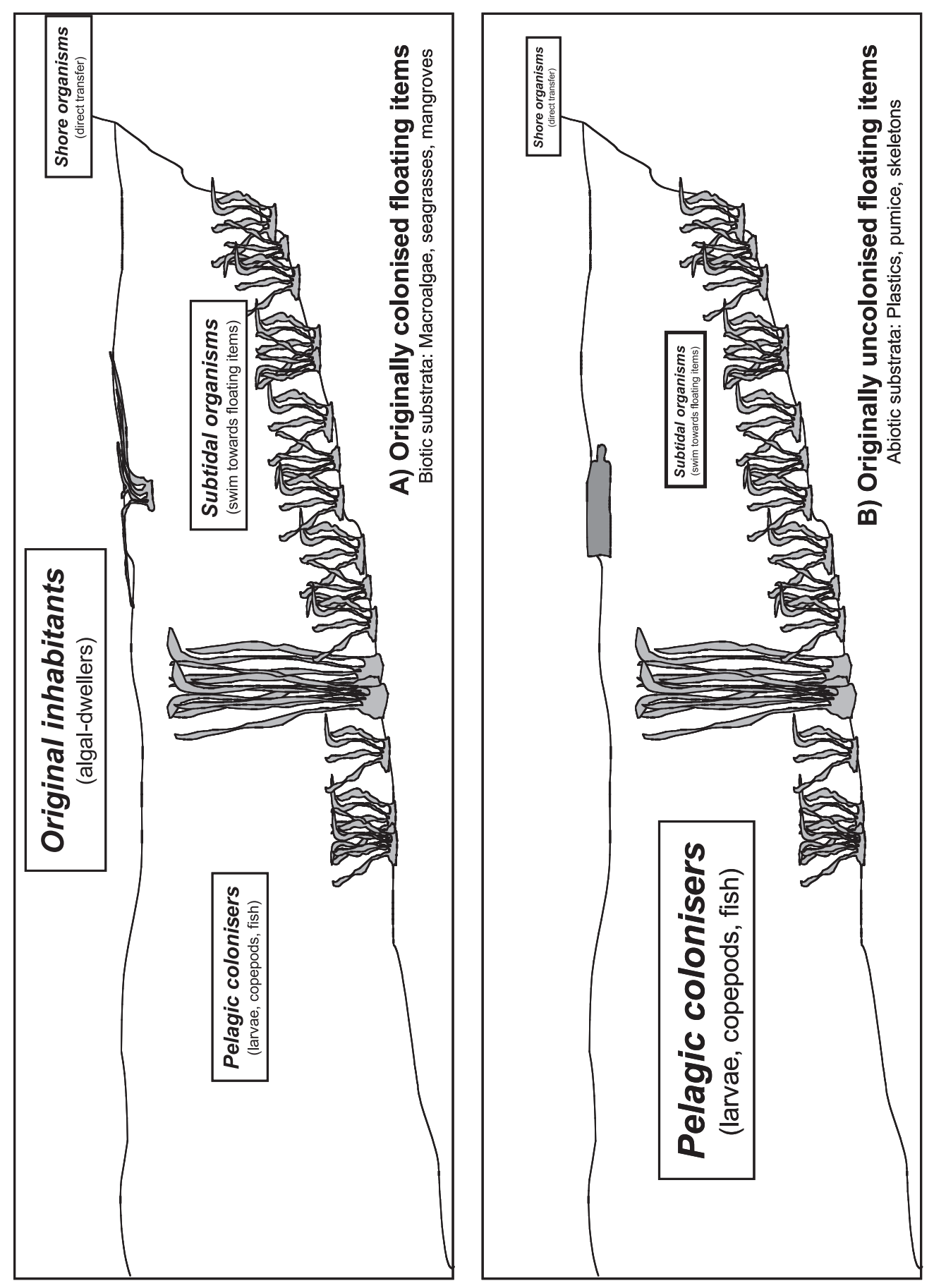

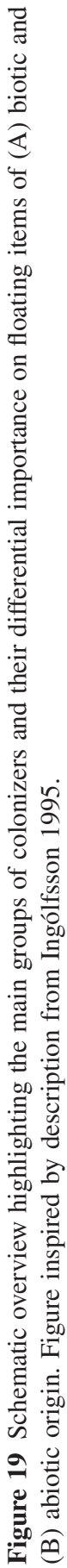




\section{MARTIN THIEL \& LARS GUTOW}

with pelagic larvae as suggested by Jokiel (1990a). In these drift rows, floating items also come in direct contact with other items and rafting organisms may move between rafts, i.e., do "rafthopping' (sensu Hobday 2000a). Hentschel (1922), discussing the species succession of rafting organisms on Sargassum had already suggested that this process (direct transfer) is more likely than transfer via pelagic stages. Direct transfer between different Sargassum plants may occur primarily during calm periods, when plants are concentrated in patches or rows (Parr 1939). This process is probably supported by internal waves that have the potential to turn scattered distributions of floating items into regular patterns (Kingsford \& Choat 1986). Following the drift of detached Macrocystis pyrifera in Monterey Bay (Southern California) by radio tracking, Harrold \& Lisin (1989) observed the incorporation of individual floating plants into large rafts of nearly $100 \mathrm{~m}$ in diameter in which the plants remained entangled for days, facilitating species exchange.

During this time, growth and ecological interactions among rafting organisms will gain increasingly in importance. Some species may overgrow others (Tsikhon-Lukanina et al. 2001a) or exert strong predation pressure on preferred prey organisms (Edgar 1987). As an outcome of these processes, organisms well adapted to the rafting environment and to the conditions on floating items will start to dominate the rafting community. Sessile rafters such as hydrozoans, bryozoans, ascidians and stalked barnacles, i.e., typical fouling organisms, can completely overgrow floating items as has been reported for plastic items (Ye \& Andrady 1991).

Following firm establishment on floating items, rafters may also start to reproduce. This is particularly necessary for obligate rafters, many of which achieve sexual maturity within very short time periods after colonising floating items (see, e.g., stalked barnacles — Skerman 1958, Patel 1959, Zann \& Harker 1978, Green et al. 1994). However, many facultative rafters also reproduce while rafting. As outlined above, of particular importance during this stage is direct development combined with local recruitment, i.e., settling in the immediate vicinity of the parents. Edgar (1987) observed a population increase for species without pelagic larval stages on holdfasts of $M$. pyrifera during the first months after detachment, and Vásquez (1993) also noted that after approximately three months of detachment peracarid crustaceans dominated in tethered holdfasts. Helmuth et al. (1994) observed a higher proportion of small individuals of the brooding bivalve Gaimardia trapesina on algal patches floating for a long time than on patches floating for a short time. Hobday (2000a) also observed that a brooding isopod increased significantly in abundance with age of the raft, and he discussed that this may be due to local recruitment and to protection from predation.

On floating items that start out their journey in a clean state, early colonisation occurs almost exclusively via pelagic stages (Figure 19). This is confirmed through experiments by Ye \& Andrady (1991) who studied the succession on plastic particles tethered in nearshore waters. Initially, a transparent slimy biofilm developed (see also Holmström \& Kjelleberg 1999), which served as precursor for subsequent settlement of algal fouling communities during the first 7 wk of exposure. During wk 9-11, hydroid colonies dominated the fouling community before they were replaced by encrusting Bryozoa and tunicates from wk 11 onwards. Tunicates finally were dominant from wk 19 on and, thus, apparently represented the final successional stage. Most plastic items have a relatively smooth surface and attachment surfaces for clinging organisms, such as amphipods, are lacking at the beginning of the rafting journey. Consequently, these items are colonised by motile organisms relatively late during the succession. Tsikhon-Lukanina et al. (2001a) observed a 30-day succession of the sessile epibionts on floating items (macroalgae, wood, plastics) in the northwest Pacific, which changed from a bryozoan-dominated to a barnacle-dominated community. The authors discussed that early stages of the species succession on floating items are characterised by bryozoans because these are able to rapidly colonise clean substrata. For the motile fauna (gastropods, polychaetes, crustaceans), they observed a change in group abundances during the 30-day succession period, with polychaetes and gastropods dominating initially, but turbellarians and crustaceans dominating after day 20. Total abundance of the motile fauna increased from 109 


\section{RAFTING OF BENTHIC MARINE ORGANISMS}

individuals $\mathrm{m}^{-2}$ at day 4 to 4170 individuals $\mathrm{m}^{-2}$ in 30 -day colonies and their biomass increased from $2.05 \mathrm{~g} \mathrm{~m}^{-2}$ to $16.13 \mathrm{~g} \mathrm{~m}^{-2}$ (Tsikhon-Lukanina et al. 2001a). This observation indicates that in a later stage of succession these motile organisms are well established and possibly reproduce on previously uncolonised floating items, similar as has been reported for floating macroalgae.

A specific succession appears to occur on Sargassum where young parts of the alga are free from epibionts (Hentschel 1922). Similar to the situation on originally uncolonised floating items, the first successional stage is characterised by rod-shaped bacteria (Conover \& Sieburth 1964). In a late successional stage, algae increasingly colonised floating Sargassum with blue-green algae followed by Rhodophyta and Chlorophyta (Conover \& Sieburth 1964). When the blades become older they are first overgrown vegetatively by hydroids, which are succeeded by bryozoans and spirorbid polychaetes. These findings were confirmed by Ryland (1974) who studied the succession on pelagic Sargassum natans collected off Bermuda. He focussed on the most common species, namely the hydroid Clytia noliformis, the bryozoan Membranipora tuberculata, the spirorbid polychaete Janua formosa and the blue-green alga Calothrix crustacea. Succession was described by analysing the composition of epibionts along the Sargassum plant with respect to the age of the different parts of the algal thallus. The youngest parts of the plants, which are located in direct vicinity of the apical growth tips of the leaflets, were densely populated by Clytia noliformis. With increasing age of the algal thallus, densities of the other three studied species increased. In the oldest parts Janua formosa, Membranipora tuberculata and Calothrix crustacea dominated, while Clytia noliformis was represented only by stolonic remnants. Calder (1995) found only minor seasonal variations in the composition of hydroid species on floating Sargassum from Bermuda, but he only collected 'medium'-aged thalli. These observations suggest that succession of floating Sargassum thalli follows a regular pattern, which is independent of season.

In general, different species dominate at different stages; in chronological order of importance is their capacity (1) to cling to floating items, (2) to colonise and compete successfully and (3) to reproduce while rafting.

\section{Successional stages}

In general, three main successional stages can be distinguished, which reflect the species succession outlined in the previous section. The initial phase where many original inhabitants of floating substrata are lost is followed by the colonisation and establishment phase where new organisms settle and establish, and the third phase where some rafters reproduce and recruit within a floating item (Figure 20). These successional stages had originally been identified based on three experimental studies (Edgar 1987, Vásquez 1993, Ingólfsson 1998) using tethered macroalgae, but other observations and short-term experiments using free-floating algae (Stoner \& Greening 1984, Kingsford \& Choat 1985, Hobday 2000a, Tsikhon-Lukanina et al. 2001a) and tethered plastics (Ye \& Andrady 1991) provided support for the validity of this conceptual model.

During the initial phase of succession there exist differences between originally colonised and uncolonised floating items. Exchange processes between original and new inhabitants dominate on colonised items (macroalgae, seagrasses and mangroves), and some of the original rafters manage to hold on to floating items and persist throughout the succession. These original inhabitants may facilitate colonisation of new organisms but can also affect recruitment of new colonisers negatively. In contrast, recruitment on previously uncolonised floating items (river driftwood, pumice, plastics) depends primarily on substratum characteristics rather than on biotic interactions. Possibly, the high proportion of sessile organisms on abiotic (i.e., originally uncolonised) substrata is a reflection of these differences.

During the colonisation and establishment phase, succession of uncolonised and previously colonised items becomes similar and many sessile organisms colonise via pelagic larvae, in particular 


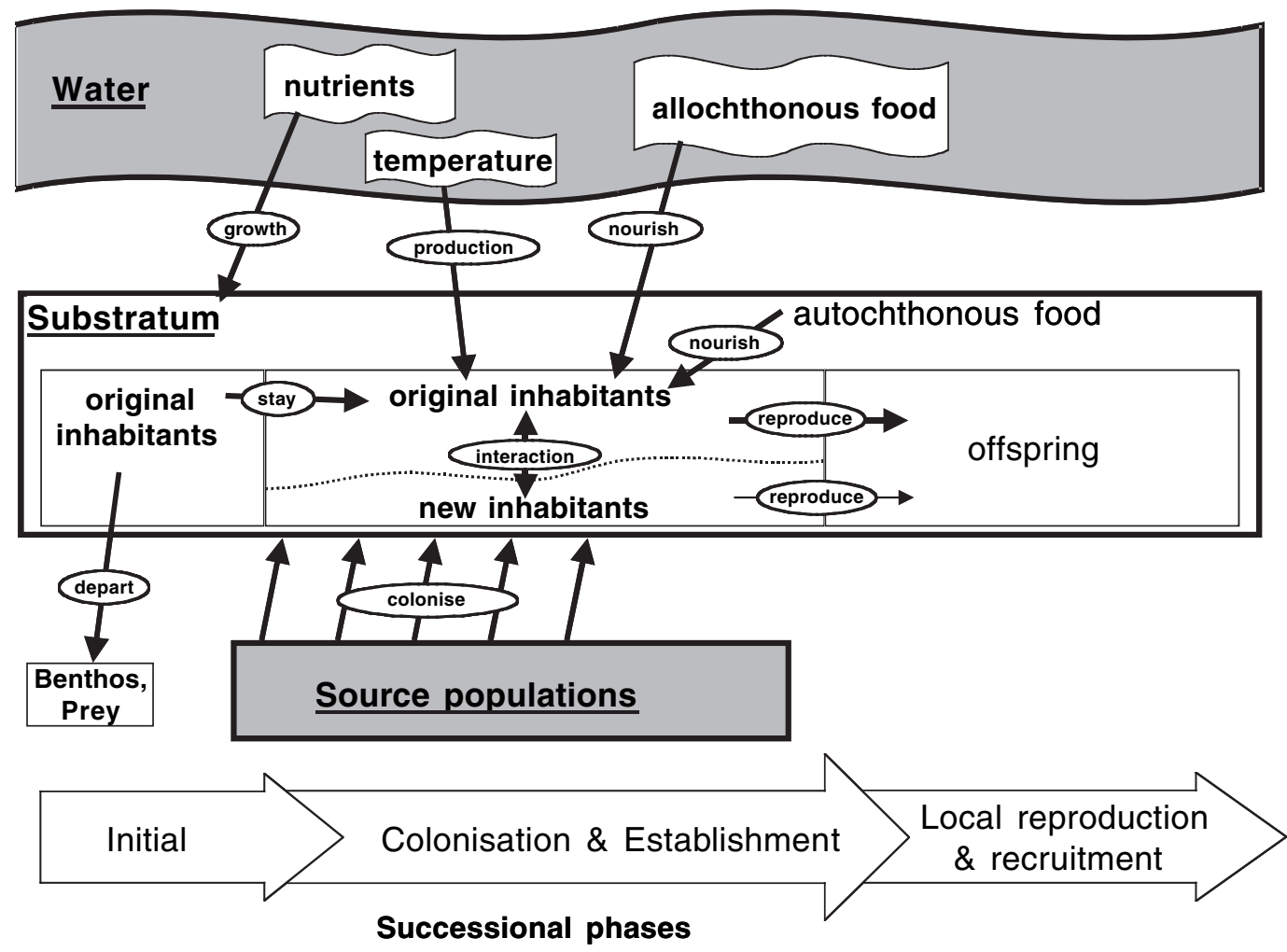

Figure 20 Successional phases of rafting community on floating items. Modified after Thiel 2003c.

when floating items leave the immediate nearshore waters. Following development of a substantial fouling community, consisting primarily of hydrozoans, bryozoans, ascidians and stalked barnacles, clinging organisms can equally be found on both originally colonised and uncolonised items. Some of these clinging organisms, which are relatively poor swimmers, may colonise floating items from benthic habitats or via 'raft-hopping'. At this later stage of rafting, local reproduction may lead to a dominance of organisms with direct development.

\section{Abundance and diversity of rafting community}

Some organisms can reach very high abundances on floating items. For example, Bushing (1994) reported between 1500-2000 ophiuroids from one single macroalgal raft. Also other authors reported very high densities for single species from individual floating items. It appears that these high densities are reached after floating items have been travelling for periods of several weeks or months. Experimental studies with suspended algal holdfasts by Edgar (1987) and Vásquez (1993) indicated that an increase in abundance of potential rafters occurs approximately 100 days after detachment. Edgar (1987) attributed an increase in total animal abundance on Macrocystis pyrifera holdfasts, about 100 days after detachment, to the quick disappearance of some of the largest holdfast dwelling species (eels, polychaetes and octopuses) probably resulting in relatively low competition and predation levels favouring the remaining fauna.

Interestingly, several studies reported that the peak in abundance of rafters occurs at some intermediate point during the rafting succession (shortly after the establishment phase), followed by a decrease in individual abundance. For example, the study by Tsikhon-Lukanina et al. (2001a) 


\section{RAFTING OF BENTHIC MARINE ORGANISMS}

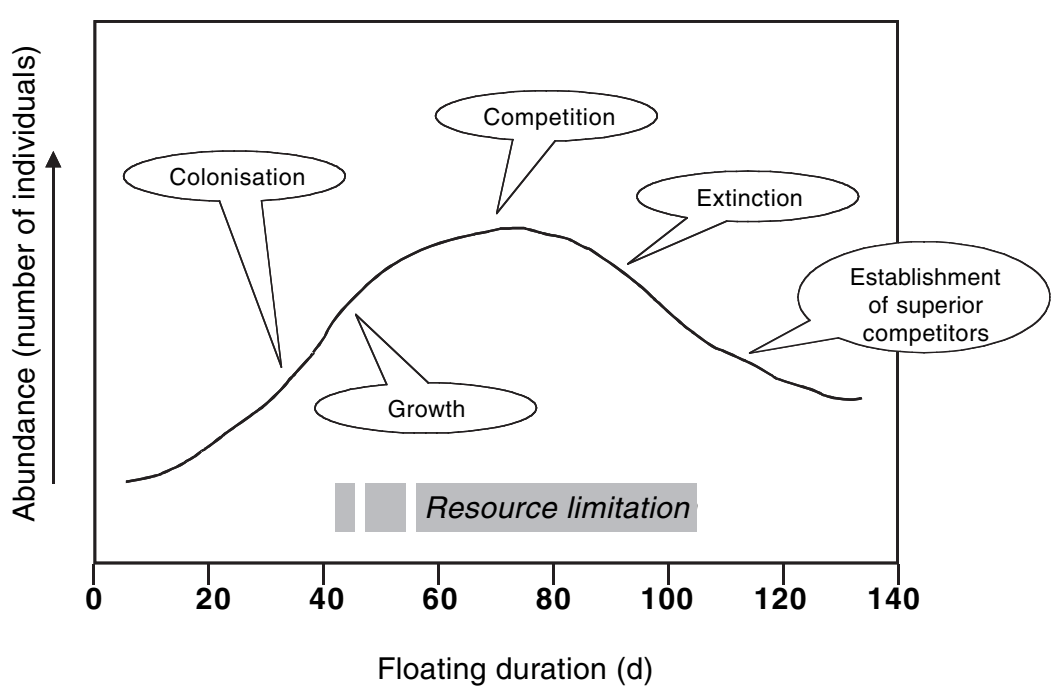

Figure 21 Changes in abundance of rafters on floating items and main processes.

revealed high densities and biomass of rafters after a floating duration of approximately 20 days (based on sizes of stalked barnacles) and a decrease between days 20 and 30. Also Edgar (1987) observed a strong decrease in abundance of rafters from 103 to 191 days after detachment. Stoner \& Greening (1984) distinguished three age classes of floating Sargassum, and they reported peak densities of rafters on the middle-aged algal patches. They remarked that "the effects of apparent clump age were more dramatic than the effects of clump size". The fact that several studies reported this decrease in abundance suggests that this could be a general trend for the rafting community. At peak densities of rafting organisms, resources (space or food) on a floating item could be depleted and only successful competitors may be able to maintain viable populations on a float (Figure 21). This pattern agrees well with the general population dynamics of species colonising a new habitat. An initial overshoot and a subsequent strong decline in population density might reflect the interaction between population growth and resource-dependent regulative mechanisms. The decline in overall numbers of rafters occurs approximately between 20-100 days after the start of the voyage. Since different species in the rafting assemblage feature different population growth rates, one could have expected that ecological conditions affect population dynamics of the diverse rafting species differentially. However, the consistent decline in abundance after the peak indicates that many rafting species experience a decline in population numbers, regardless of their species-specific population growth rates. This strongly suggests that important ecological constraints affect all species in a similar manner. Based on the above arguments, we consider it most likely that resource limitation (space, food) is responsible for the observed decline in abundance. If rafters indeed exhaust resources at this stage, then one could expect that after passing this peak during the succession, biotic items may experience dramatic losses in buoyancy. In contrast, abiotic items that are not consumed by rafters may persist at the surface of the sea and competitive interactions may lead to the establishment of a community with one (or few) dominant species.

As a result of loss of species and dominance of persistent rafters, one should also expect a decrease in species diversity after these peaks in abundance of rafters. High species diversity can be reached early during rafting (Tsikhon-Lukanina et al. 2001a). Winston et al. (1997) also suggested that middle-aged plastic items had the highest species richness of rafting organisms. Hobday (2000a), however, found no significant relationship between age of floating macroalgae and species richness of rafters. 


\section{MARTIN THIEL \& LARS GUTOW}

Given successional changes during rafting, it can be expected that rafting communities differ substantially from benthic communities. Several studies have examined these differences for floating macroalgae, which have their origin in benthic habitats. For example, all giant kelps (e.g., Macrocystis spp., Nereocystis luetkeana, Pelagophycus porra and Durvillaea antarctica) but also smaller brown algae (Sargassum spp., Fucus spp. and Ascophyllum nodosum) are initially colonised by flora and fauna while growing attached to benthic substrata before becoming detached and being converted into floating substrata. Given that these benthic algae are inhabited by a diverse assemblage of benthic organisms (Ojeda \& Santelices 1984, Smith \& Simpson 1995, Thiel \& Vásquez 2000), and that after detachment these algae together with their inhabitants may become dispersed via oceanic currents, it is of great interest to know how rafting communities of these algae differ from their benthic counterparts.

Differences between attached and floating macroalgae have been examined either experimentally or empirically. A cross-study comparison revealed that members of all groups might occasionally occur in higher numbers on floating algae than on benthic algae (Table 18). While no clear taxonomic trend is recognisable, some tendencies are worthy of mention. With the exception of Durvillaea antarctica, on all macroalgae at least some faunal groups increased after algae had gone afloat. This means that some taxa may find favourable conditions on floating macroalgae as compared with benthic counterparts. The fact that all taxa experienced losses on $D$. antarctica suggests that benthic plants of this species provide a better habitat for associated fauna than do floating plants. Possibly, the compact holdfast of this alga provides few attachment sites for fauna, while complex holdfasts such as those from Macrocystis pyrifera offer refuges for a diverse community, even after going afloat. Edgar (1987) remarked that "the protection provided by several layers of haptera presumably protects the fauna residing within the (M. pyrifera) holdfast from much of the external water-turbulence". Differences in faunal composition between benthic and floating algae do not always depend on holdfast characteristics, as is underlined by the fact that several taxa also increased in abundance on floating Ascophyllum fronds (Ingólfsson 1998) (Table 18). A consistent tendency to colonise floating macroalgae has been revealed only for megalopae of decapod crustaceans, which apparently actively seek out floating substrata in nearshore waters (see also above). Positive tendencies were also observed for adult decapods and fishes. In contrast, arachnids, gastropods and oligochaetes appeared in consistently lower abundance on floating than on benthic macroalgae. Between-study comparisons also suggest that ecological interactions might cause the differences in faunal composition between benthic and floating algae. For example, Kingsford \& Choat (1985) observed that abundances of harpacticoid copepods were much lower on floating than on attached macroalgae while fish abundance showed an inverse pattern, suggesting that fish predation could contribute to the absence of harpacticoid copepods on floating algae. In contrast, Ingólfsson (1998) reported a pronounced increase of harpacticoids on floating algae. Fish predators also prey on fauna associated with floating algae around Iceland (Ingólfsson \& Kristjánsson 2002) but predation pressure may not be as high as in New Zealand waters.

For the Gulf of Mexico, Hedgpeth (1953) also mentioned that the fauna from holopelagic Sargassum differed substantially from that on detached benthic Sargassum. The most consistent deviations are the absence of the Columbus' crab Planes minutus from detached plants and the occurrence of the pycnogonid Tanystylum intermedium, which is lacking on holopelagic plants. Detailed reasons for these differences are not mentioned. Weis (1968) found gastropods from the genera Bittium and Rissoa on floating Sargassum and she discussed a benthic origin for these but provides no further information.

Thus, differences between faunal composition on benthic and floating substrata are primarily due to substratum characteristics, the biology of associated fauna or ecological interactions during floating. The analysis conducted herein represents a rather crude taxonomic comparison. We suggest 
RAFTING OF BENTHIC MARINE ORGANISMS

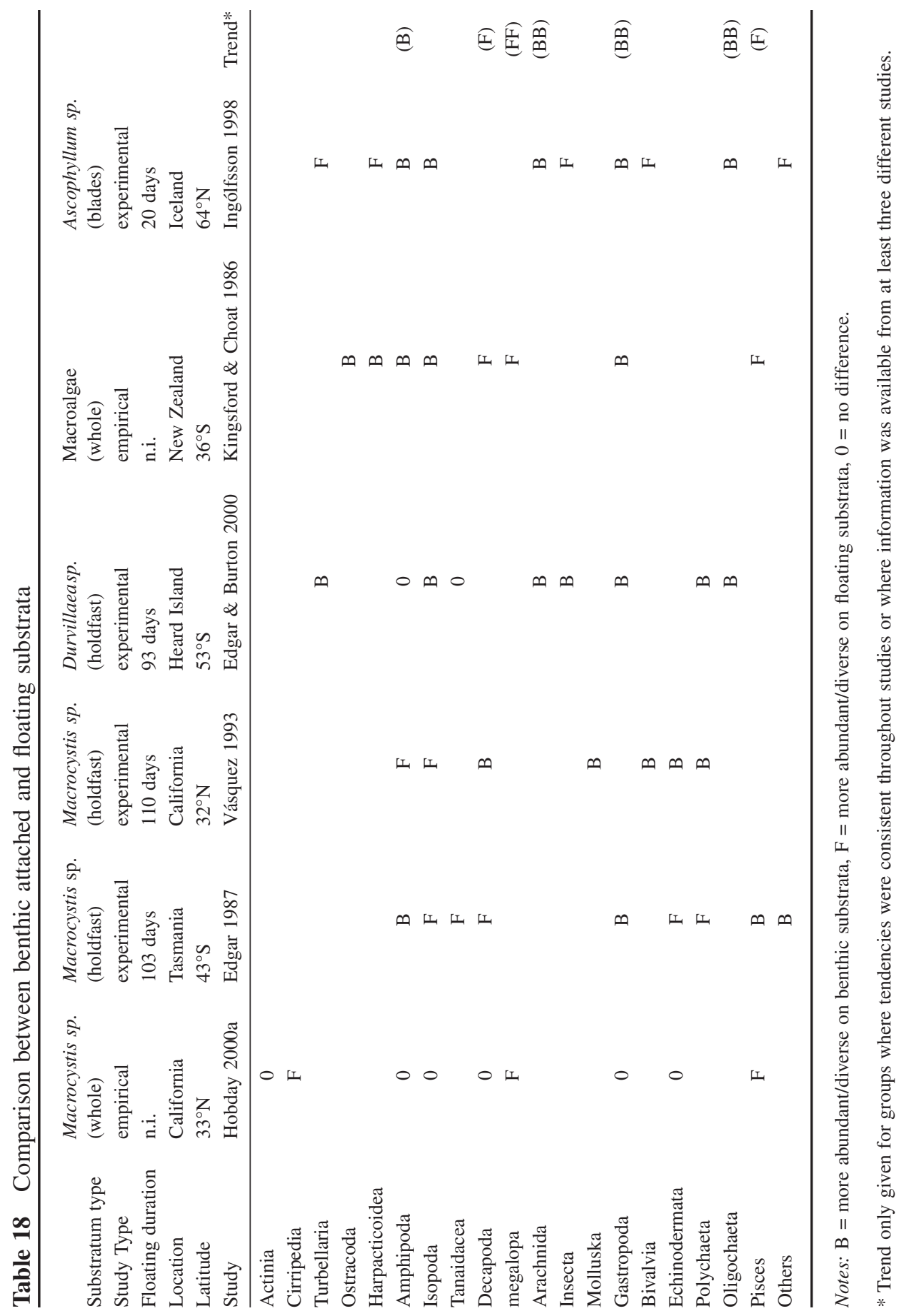




\section{MARTIN THIEL \& LARS GUTOW}

that future comparisons should focus on biological traits of rafting organisms and on ecological interactions.

\section{Geographic and seasonal variations in rafting communities}

The abundance and species composition of rafting communities varies throughout the world's oceans. This is partly due to the global variations in availability of different rafting substrata (see Thiel \& Gutow 2004). However, there are also regional differences in other environmental variables (e.g., nutrient availability, primary productivity and temperature) that affect the presence and persistence of rafting organisms on floating items.

Regional differences in the Sargassum community had been recognised early on. Timmermann (1932) emphasised that the northern part of the Sargasso Sea, i.e., the area of the Gulf Stream, features a higher diversity of rafting organisms than the southern part. Conover \& Sieburth (1964) revealed that bacterial population densities on floating Sargassum in the North Atlantic varied geographically according to the specific production of chemical antifouling mechanisms. Stoner \& Greening (1984) provided a multifactorial comparison of the fauna associated with floating Sargassum from the Sargasso Sea and the Gulf Stream and found only low similarities between faunal assemblages from these regions. Sargasso Sea samples showed a relatively even distribution of species, with the gastropod Litiopa melanostoma dominating the community with a mean proportion of about $25 \%$ of the total fauna. Gulf Stream samples, however, were slightly less diverse and strongly dominated by the shrimp Latreutes fucorum comprising about $70 \%$ of the Sargassum fauna in that region. Consistently, the Gulf Stream fauna yielded a higher percentage of large omnivores (sensu Butler et al. 1983), while in the Sargasso Sea smaller omnivores consuming primarily sessile prey organisms were more abundant. Similarly, Smith et al. (1973) found higher numbers of rafting species in the Slope Water (33 species) than in the Sargasso Sea (about 25 species). However, densities of the animals in the Sargasso Sea ( 4200 ind. $\mathrm{g}^{-1}$ wet weight [WW] Sargassum) were about twice as high as in the Slope Water ( 2000 ind. $\mathrm{g}^{-1}$ WW Sargassum). In the same region, Keller (1987) found that the biomass of the three most abundant invertebrate species was linked to the nutrient content of the surrounding waters. High epifaunal biomass was observed in the centre of the nutrient-rich, cool slope waters north of the Gulf Stream while productivity was low in the oligotrophic waters of the Sargasso Sea. Unexpectedly high productivity of epifauna was detected in the Sargasso Sea in the track of a cold water ring from the Gulf Stream. The nutrientloaded subarctic slope water allowed for high primary production inside the ring influencing even the productivity of higher trophic levels such as the motile carnivorous epifauna on floating Sargassum. Niermann (1986) suggested that the low degree of epibiont coverage (primarily bryozoans and hydrozoans) on floating Sargassum in the Sargasso Sea proper could also be due to food limitation.

Data presented by Winston et al. (1997) first hinted towards a latitudinal decrease in colonisation of marine debris by sessile rafters, which was later confirmed by studies from Barnes \& Sanderson (2000). In general, items from low latitudes are densely colonised while items from high latitudes are typically uncolonised (Winston et al. 1997, Barnes \& Sanderson 2000, Barnes 2002). While there exist latitudinal variations in abundance and type of floating substrata (Barnes 2002, Thiel \& Gutow 2004), differences in colonisation appear to be primarily due to ecological factors. In polar regions sessile rafters (primarily bryozoans) were almost completely absent from floating debris (Figure 22), and the authors discussed that this could be due to the harsh physical conditions at high latitudes (Barnes \& Sanderson 2000, Barnes 2002).

Seasonal variations of the abundance of rafting organisms are also more pronounced at high latitudes (Figure 23). During the winter, many floating items collected in Icelandic waters $\left(64^{\circ} \mathrm{N}\right)$ were almost free of rafters. In particular, the occurrence of some colonisers on floating seaweed 


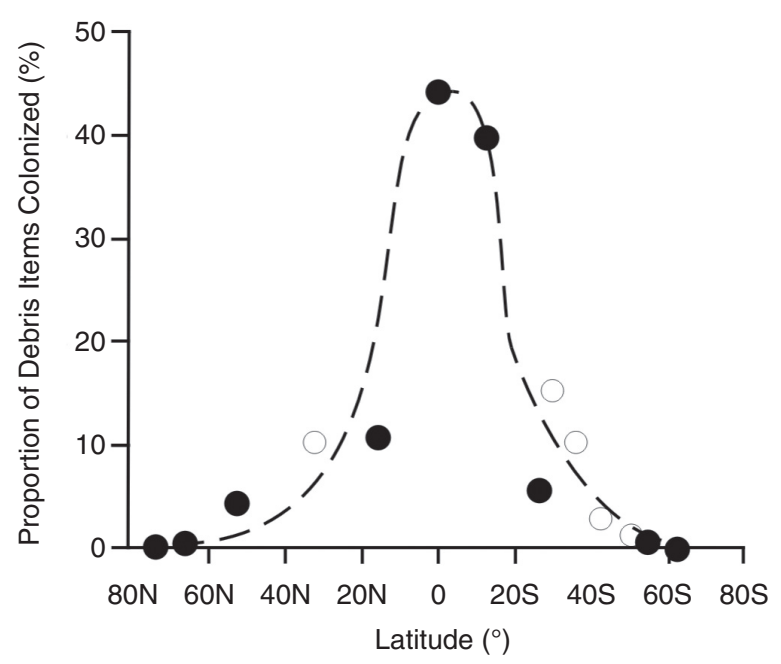

Figure 22 Latitudinal variation in proportions of floating debris colonized by sessile organisms. Modified after Barnes \& Sanderson 2000.

was highly seasonal (Ingólfsson 2000). Common rafters such as the amphipods Calliopius laeviusculus, Ischyrocerus anguipes and Dexamine thea as well as the copepod Parathalestris croni showed sharp seasonal peaks in abundance. In others, such as Gammarellus angulosus, the lumpsucker Cyclopterus lumpus and the isopod Idotea baltica, the highest yearly abundances were less pronounced. Maximum densities of rafting peracarids occurred during the middle of summer, when local populations of these species were characterised by relatively large proportions of juveniles. Rafting organisms were virtually absent from floating algae in winter. Probably, most animals avoid extreme surface temperatures in the North Atlantic by leaving the neustonic habitat actively in winter and descending to the ground as described for I. baltica in the Bay of Fundy (Locke \& Corey 1989). Seasonal variations of the rafting community are less pronounced at lower latitudes (Figure 23). For macroalgae from Californian waters $\left(33^{\circ} \mathrm{N}\right)$, Hobday (2000a) could not identify clear seasonal trends - some rafting species were more common in winter, while others increased in abundance during the summer months. For hydroid communities collected on floating Sargassum near Bermuda $\left(\sim 32^{\circ} \mathrm{N}\right)$, Calder (1995) also found no regular seasonal succession as is known from temperate estuaries. Other authors, however, reported a decrease in faunal abundance on floating Sargassum towards the winter months (Timmermann 1932). Winston et al. (1997) also observed that the proportion of colonised floating plastics collected on the Florida Atlantic coast was slightly higher in summer than in winter. For the fish fauna associated with floating objects off Kyushu (Japan, Korea Strait), Uchida \& Shojima (1958) revealed a strong seasonal trend with low abundances in winter. Another study from the northwest coast of the Korea Strait confirmed this seasonal pattern (Cho et al. 2001). Possibly, the strong seasonal trend observed in that area is due to the fact that common floating items in these waters (floating macroalgae) also appear abundantly in summer (see Thiel \& Gutow 2004). This is supported by observations from Kingsford (1992) who reported high abundances of fish on floating macroalgae during the summer months (Figure 23), when algae themselves were also more abundant.

In summary, global and seasonal trends in the distribution and appearance of rafting communities appear to be linked to the availability of floating substrata and environmental factors such as temperature and nutrient availability. Not unexpectedly, seasonal trends are more pronounced at high latitudes than at low latitudes. While items at low latitudes support rafting assemblages throughout the year, at high latitudes rafters disappear from floating items during the winter months 
MARTIN THIEL \& LARS GUTOW

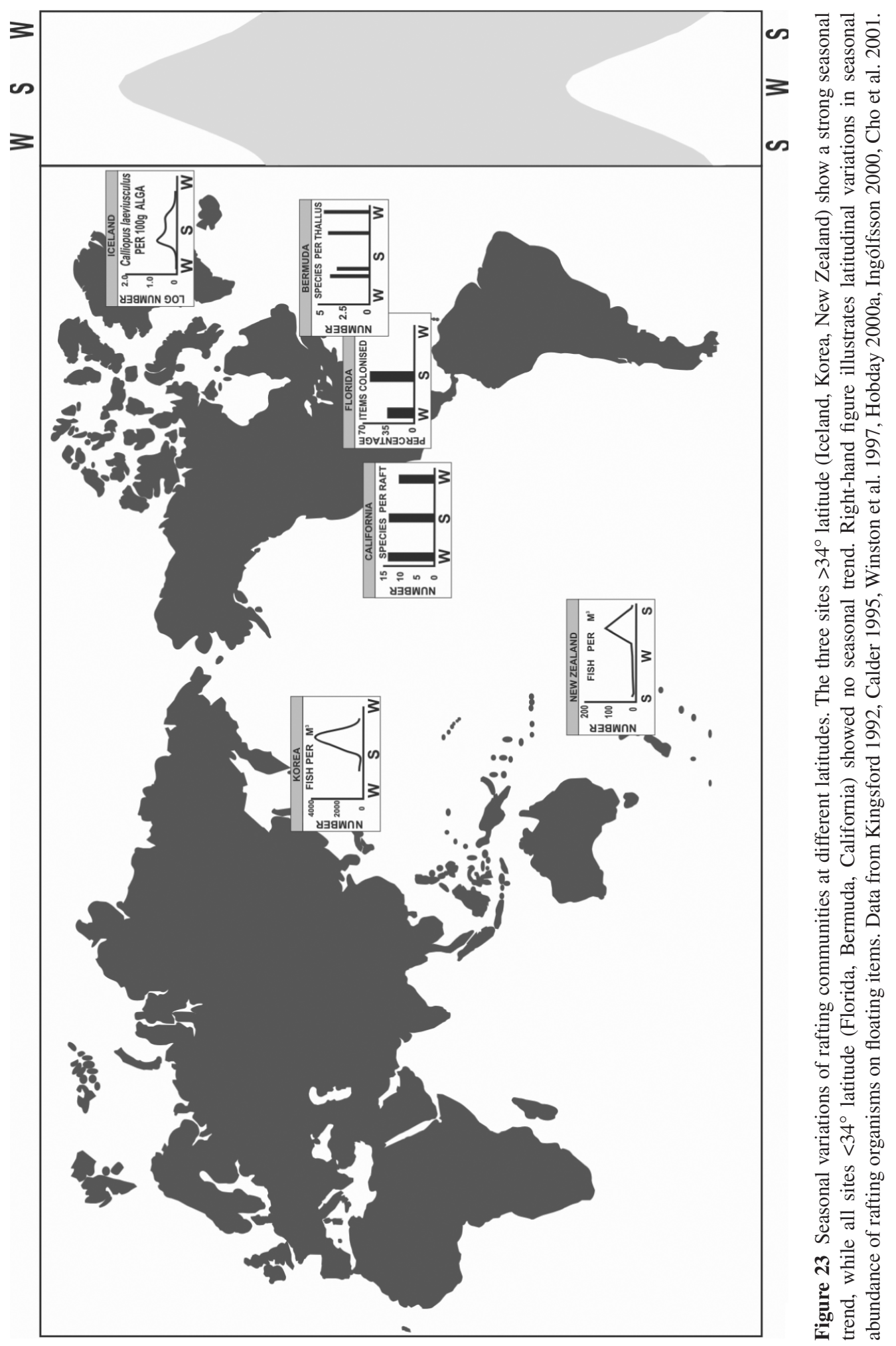




\section{RAFTING OF BENTHIC MARINE ORGANISMS}

(Figure 23). Based on the results from a previous review on the global distribution of floating items (Thiel \& Gutow 2004), we suggest that rafting dispersal via macroalgae will be most important around mid latitudes (see also Highsmith 1985, Kingsford \& Choat 1985), especially between 34 and $20^{\circ}$ latitudes in both hemispheres, where floating macroalgae may be colonised throughout the year. Wood is most common in polar arctic and in equatorial tropical regions (Thiel \& Gutow 2004) but rafting may be temporally limited in the arctic region because of the observed seasonal patterns in colonisation. The global pattern in rafting opportunities may have important implications for the biodiversity of coastal communities in these regions.

\section{Arrival and colonisation processes}

Arrival and subsequent colonisation are the most crucial steps in the rafting circuit because rafters need to disembark and furthermore establish in a new environment. The process of colonisation of new habitats by a rafting organism must follow the same sequences as that of colonisation by anthropogenically introduced species (or any coloniser for that matter), namely, arrival, settlement, expansion and persistence (Ribera \& Boudouresque 1995). Clearly, arrival of rafting organisms in nearshore environments must happen frequently since many floating items are permanently cast ashore all along the coasts of the world's oceans. Surprisingly little, though, is known about this final step of a rafting journey. The major reason for this lack of information could be the difficulty in observing or studying colonisation processes. In most cases the arrival of rafters may be singular events and the probability for an observer of being at the right place at the right time is low. Arrival and successful colonisation of rafters, which are typically small (see above), may have gone unnoticed for a long time. Establishing persistent (and for an observer, conspicuous) populations at a new site can be expected to be a process lasting several generations. Consequently, once a coloniser is perceived it is difficult to infer the transport mechanism, but it is even more complicated to elucidate the colonisation process.

Despite these difficulties and the low probabilities of witnessing these events there are a few reports on the arrival of rafters. Worcester (1994) studied the arrival and successful recruitment of a clonal ascidian (Botrylloides sp.) that had rafted on seagrass blades. After arriving on a floating seagrass blade, colonies either moved onto attached plants or grew asexually onto the new substratum. Arrival of natural and experimentally produced rafts was monitored by this author on several occasions (Worcester 1994). One of the most exciting published reports on arrival of rafters, though, is on green iguanas, which apparently had travelled on a patch of logs and uprooted trees to a Caribbean island previously not inhabited by these lizards (Censky et al. 1998). Since several individuals of both sexes arrived during this event, the authors suggested that the chance for successful colonisation was high. They saw this suggestion confirmed by the capture of a sexually mature female 29 months after first arrival of the iguanas.

Despite the fact that these two species could hardly be more different, these two reports on arrival of a sessile compound ascidian and of a highly motile reptile illustrate two important requisites for successful arrival and colonisation: (1) to disembark from floating items and (2) to persist and establish viable populations at the arrival site.

\section{Arrival and debarkation}

Sessile organisms, when carried on their rafts into nearshore waters after a long oceanic journey, have only a limited capacity to detach and reattach. For example, Patel (1959) carefully detached stalked barnacles Lepas anatifera from their original substratum and maintained them healthy for several weeks in glass dishes with sea water but did not observe any reattachment. It can be safely assumed that most fully sessile rafters cannot reattach to benthic substrata in coastal habitats. 


\section{MARTIN THIEL \& LARS GUTOW}

However, as pointed out above, many organisms reproduce during the rafting journey, and it is very possible that rafters release offspring when approaching nearshore waters. This mode of 'en route' release of dispersal stages has been invoked for some rafting hydrozoans (Cornelius 1992a), bryozoans (Keough \& Chernoff 1987) and other clonal organisms (Jackson 1986, Jokiel 1989). Spore-release has also been reported for detached macroalgae Macrocystis pyrifera floating at the sea surface (citations in Coyer et al. 2001) and Miller et al. (2000) discussed that sorus-bearing sporophytes of Pelagophycus porra may be important in dispersal of that species along the California coast. Deysher \& Norton (1981) suggested that plants of Sargassum muticum might become fertile and reproduce during their voyage, i.e., 'en route'. This had also been reported for epiphytic (rafting) algae growing on Sargassum (Oliveira et al. 1979). In Botrylloides sp. Worcester (1994) noticed that within a few weeks after arrival and attachment of large founder colonies in a previously uncolonised seagrass bed, small patches $(2 \mathrm{~m}$ diameter) with many small colonies developed, probably from larvae released from the founder colonies that had arrived via rafting. Keough \& Chernoff (1987) also suggested that floating colonies of Bugula neritina that pass over seagrass meadows release larvae, 'providing a pulse of settlement'. Possibly, many fully sessile rafters dump propagules when approaching or making contact with benthic habitats in nearshore environments (Figure 24). Temperature differences between offshore and coastal waters (Kaplan et al. 2003), known to trigger propagule release (Bonardelli et al. 1996), may facilitate this process.

Another interesting proposal had been made by Jokiel (1989) for rafting corals. He suggested that "on atolls and many reefs, the incoming flotsam must traverse the reef flat", and upon scraping along the reef flat, organisms (e.g., coral colonies) may fall off and continue to grow in benthic habitats. While many organisms may not survive this form of debarkation, cnidarians have a high regeneration potential and may regrow after detachment. Reattachment and continuing growth had also been mentioned by Hoffmann (1987) for some macroalgae.

Some semi-sessile organisms may be able to let go from rafts and subsequently reattach to benthic substrata. In this context, Häussermann \& Försterra (2001) mentioned the remarkable ability of the sea anemone Anemonia alicemartinae to detach and reattach very rapidly. Many sea anemones have this capacity (e.g., Shick et al. 1979, Anthony \& Svane 1995, Houtman et al. 1997), albeit there apparently exist species-specific differences in motility (see above). Also, byssal-producing mussels can release hold from the original substratum and reattach to new surfaces, as has been discussed by Horvath \& Lamberti (1997) for rafted individuals of the zebra-mussel Dreissena polymorpha. Tube-dwelling polychaetes and amphipods may also abandon their homes occasionally and construct new mucus-tubes in the vicinity, i.e., on benthic substrata. Thus, many semi-sessile organisms can move from rafts onto attached benthic substrata when arriving in coastal habitats.

Debarkation will be most efficient in fully motile rafters well adapted to hold firmly to a substratum but to let go periodically in order to move to new areas. Crustaceans with efficient clinging capability may be well suited to 'jump shore' after arrival. Peracarid crustaceans, for example, have been found to crawl easily from one alga to another (Taylor 1998). Epiphytic amphipods show high turnover rates in seagrasses and algae from benthic habitats (Edgar 1992, Buschmann 1990, Taylor 1998) and the ability to move rapidly between algae (Brooks \& Bell 2001b) may facilitate colonisation of new habitats after arrival. Strong exchange in the coastal algal belt is achieved by efficient clinging and crawling interspersed with brief swimming bouts (e.g., Ingólfsson \& Agnarsson 2003). It can be assumed that many other crustaceans (harpacticoid copepods and brachyuran decapods) are similarly well adapted to move from floating items to benthic habitats. Many gastropods are also characterised by a high motility, even though many species are not well adapted to maintain attached in exposed habitats. There may be environmentrelated differences in efficiency of successful debarkation of gastropods. Also, as has been pointed out above, rafting of many gastropods may occur via attached egg masses, and when floating items become entangled and anchored in coastal habitats, juvenile snails emerging from these egg masses 


\section{RAFTING OF BENTHIC MARINE ORGANISMS}
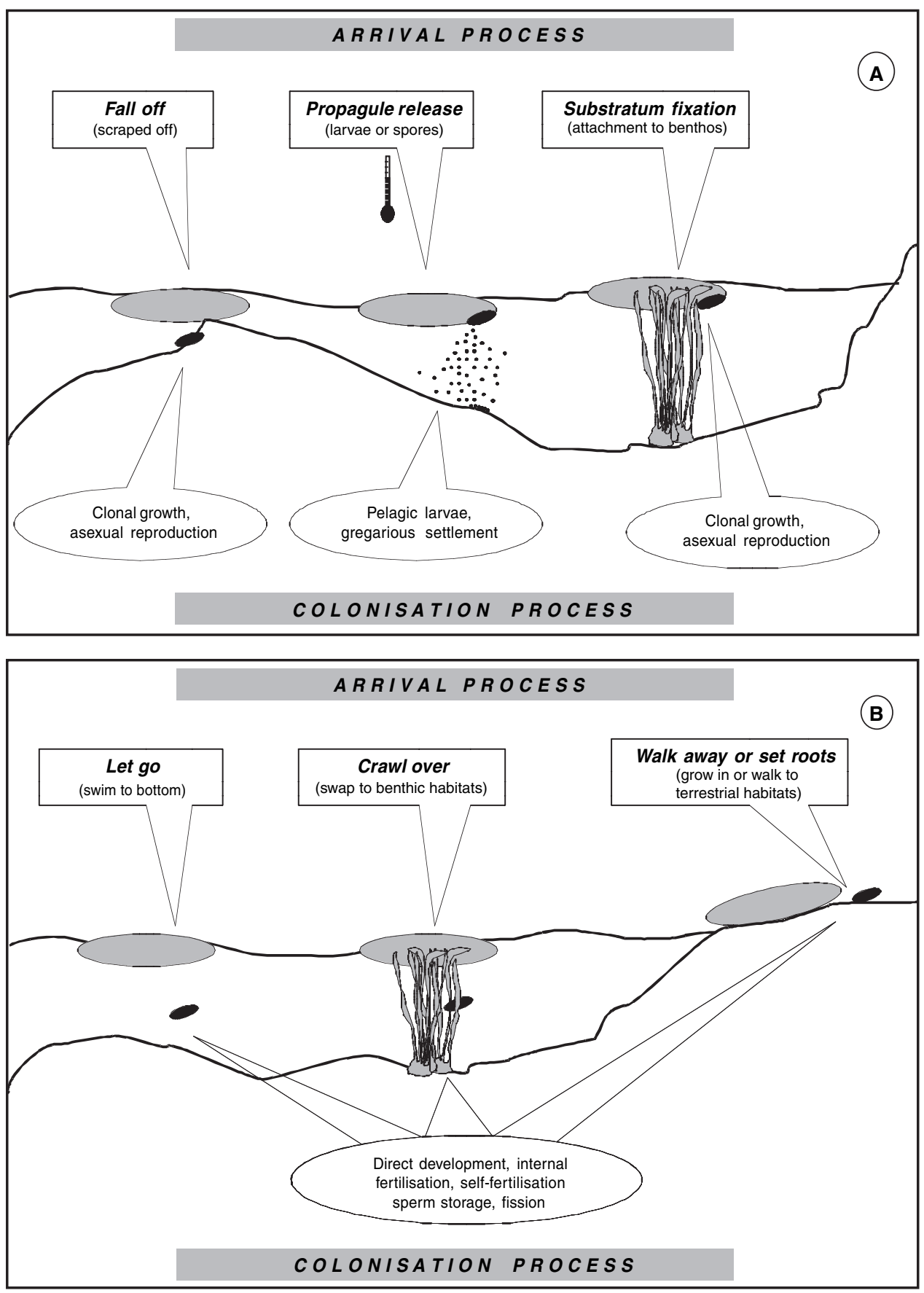

Figure 24 Schematic overview of arrival process and reproductive features of rafting organisms that favour successful colonisation in (A) sessile rafters and (B) motile rafters.

may crawl onto benthic substrata. In summary, arrival proceeds very differently for sessile, semisessile and motile organisms (Figure 24), with the latter being the most efficient in disembarking from floating items. 


\section{MARTIN THIEL \& LARS GUTOW}

\section{Survival and establishment after arrival}

It has been recognised early on that arrival in a new habitat may not be sufficient for successful colonisation. Barbour (1916) remarked that snakes Boa constrictor may have repeatedly been carried to islands off the mouth of the Orinoco, but successful establishment never occurred. Similarly, Hentschel (1922) observed that large quantities of Sargassum spp. overgrown with the hydroid Aglaophenia latecarinata arrived in the Bermudas but attached Sargassum spp. from Bermudan coasts did not harbour this hydroid. Following arrival and debarkation in a new environment, an organism needs to survive and reproduce successfully, because otherwise rafting would not become an ecologically and evolutionary significant process. Survival will be most successful if environmental conditions at arrival sites are similar to those in source regions. Evaluating species invasions to North American marine habitats, Ruiz et al. (2000) remarked that "for example, tropical species arriving in polar ecosystems may experience very poor survival compared to those arriving in temperate or tropical ecosystems". These authors also referred to the importance of salinity at arrival sites. Arnaud et al. (1976) emphasised that beyond allowing mere survival, the conditions in new habitats also need to be favourable for reproduction. Similarly, Brandon-Jones (1998) pointed out that "for successful rafting, landfall must be accompanied by an appropriate climate. Rainforest flora and fauna on a raft will not flourish on arrival unless the raft lodges at a locality with adequate precipitation and temperature". This author also discussed the concept that successful dispersers are expected to be comparatively well able to cope with a range of climates and perhaps be pre-selected after enduring a rafting journey, including exposure to sea water and low food availability. In this context, it appears safe to assume that many organisms carried with the Gulf Stream from the Caribbean to northwest Europe might not be capable of establishing viable populations. Even though seeds of tropical plants frequently reach northwest-European shores (Guppy 1917), successful germination or survival of young plants usually is inhibited by the low winter temperatures in this region. The regular occurrence of the rafting isopod Idotea metallica in the North Sea appears to be related to a long-term increase in summer temperatures in that region. This species is frequently transported to that region via rafting, but establishment of a persistent local population is prevented by elimination during winter because of temperatures too low for reproduction (Gutow \& Franke 2001). Green et al. (1994) for a stalked barnacle and Jara \& Jaramillo (1979) for a rafting crab also suggested that low temperatures may have suppressed reproductive activity in these obligate rafters.

Besides these factors, the degree of wave exposure and the substratum characteristics (soft bottom or hard rock) that organisms encounter upon arrival are of importance. Most hard-bottom inhabitants will be doomed when stranded on a sandy beach. In this context it is interesting to note that Grantham et al. (2003) remarked that the proportion of rafting species was comparatively low in sandy subtidal habitats (compared with the other studied habitats: rocky subtidal and rocky and sandy intertidal habitats). Besides abiotic factors, rafters will also be exposed to biotic interactions after arrival in new habitats. Their capacity to withstand predation pressure and to compete successfully (for food and space) will determine their survival success. Environmental requirements may be particularly important in the case of organisms that cannot move in search of suitable habitats after arrival at a new shore.

After having disembarked and survived, successful reproduction is essential in order to establish persistent populations. With respect to the reproductive biology, a good coloniser features similar traits as a good rafter, namely, the capability for self-fertilisation, internal fertilisation, asexual reproduction or direct development. Most terrestrial species feature sexual reproduction and consequently their capability of successful colonisation may depend on the availability of a mating partner. Many authors remark the necessity of simultaneous arrival of both sexes of a sexually reproducing species (e.g., Dahl 1984). In this context it is interesting to note that Heatwole \& 


\section{RAFTING OF BENTHIC MARINE ORGANISMS}

Levins (1972) often found several conspecific individuals of terrestrial invertebrates on a floating item, which coincides with the report by Censky et al. (1998) on the simultaneous arrival of several individuals of the green iguana on the beaches of the Caribbean island Anguilla (see above). It might be that rafting of terrestrial species occurs very rarely but when it occurs there might be a high likelihood that several conspecifics travel together on a large floating item (see also previous section).

However, even in species with sexual reproduction, successful colonisation may occur without the need of males and females to arrive simultaneously in a new habitat. In many species, e.g., some crustaceans (Franke 1993, Hartnoll 2000) and gastropods (Giese \& Pearse 1977 cited in Cunningham \& Collins 1998, Martel \& Chia 1991), females are capable of storing sperm or they brood their offspring. Arrival of a fertilised or gravid female that carries sperm (either unused stored sperm or in form of male offspring) may be sufficient for colonisation of new habitats. Wheeler (1916) discussed that "of course, the establishment of a species of ant on an island does not require the conveyance of a whole colony as in this instance, since a single fecundated female enclosed in a vegetable cavity might, if transported in a similar manner, suffice for the establishment of a species". Heatwole \& Levins (1972) reported the finding of an ant colony including a queen (which might have carried stored sperm from previous copulations) on a floating object $9 \mathrm{~km}$ from the next shoreline. Referring to lizards from Caribbean Islands, Fox (1963) discussed that sperm storage "may also be of significant survival value in submarginal habitats where males may be scarce", i.e., on a remote shore where a solitary female lizard disembarked from a raft.

Many brooding species of the small bivalves from the genus Lasaea are apparently capable of self-fertilisation (Ó Foighil \& Eernisse 1988). Consequently, arrival of a single individual might be sufficient to establish a new population. Skarpaas \& Stabbetorp (2001) pointed out that the shingle beach plant Mertensia maritima, which has an amphi-Atlantic distribution, is self-compatible and capable of self-pollination. Carlquist (1967), who remarked on the high degree of littoral flora thought to be dispersed via floating seeds (or plants), also noted that many of these species are self-fertile. Similarly, Sargassum muticum is monoecious and most likely self-fertile and thus single plants or fragments can give rise to whole new populations (Norton 1976). In this context it should also be remarked that Hentschel (1922) reported Sargassum spp. to be vegetative throughout the Sargasso Sea. The fact that pelagic $S$. fluitans and $S$. natans are mostly vegetative has been confirmed by other authors, but Parr (1939) mentioned the finding of a fertile $S$. fluitans attached to a small piece of floating wood. Norton \& Mathieson (1983) remarked that detached macroalgae mostly grow in the vegetative state and do not become reproductive after detachment, neither do they reattach to the bottom (but see Hoffmann 1987). Smith \& Walters (1999), however, reported that even very small fragments of Caulerpa taxifolia are capable of building rhizoids and thus are able to reattach. Populations of macroalgae and seagrasses thought to have originated from floating plants commonly feature no or only few reproductive plants (e.g., Mathieson et al. 1982, González \& Edding 1990a, Albrecht \& Reise 1994, Reusch et al. 1999). Possibly, rafting-related processes and higher colonisation potential select for vegetatively growing or self-fertile organisms.

Rafting species with direct development are also favoured over species with pelagic larvae since their offspring are capable of settling immediately (or shortly) after release from the parental brood structure. Thus, upon arrival either parents or their newborn offspring may colonise new habitats since they feature all traits necessary for successful settlement. Pelagic larvae, however, can settle only during specific periods of their larval life, i.e., during the competent phase. If larvae reach new habitats outside their competent phase, they may not be capable to delay metamorphosis (e.g., Gebauer et al. 2003) and no successful settlement may occur. This aspect may be particularly important when dispersal occurs over wide ocean expanses as compared with dispersal in an alongshore direction where larvae may have repeated chances to encounter suitable habitats. Palmer \& Strathmann (1981) emphasised that pelagic larvae may pass many suitable settlement sites before 


\section{MARTIN THIEL \& LARS GUTOW}

reaching the competent stage - this may occur to a much lesser extent in species with direct development that are dispersed on floating objects, where both adults and offspring can be considered to be always competent to settle onto newly encountered habitats. For the genus Lasaea, Ó Foighil (1989) summarised in this context that species with direct development have equal abilities of long-distance rafting as species with planktonic larvae given that both have access to floating substrata. However, species "that lack pelagic larvae are much more adept at turning rare rafting events into successful colonisation of new sites". Furthermore, pelagic larvae are easily dispersed away from the place of release by currents leading to a significant dilution of the population and, thus, to a subsequent decrease in the probability of successful mating after first colonisation. Johannesson \& Warmoes (1990) pointed out that the high colonisation potential of the directly developing Littorina saxatilis might be responsible for rapid recolonisation of previously defaunated areas (see also Wilhelmsen 1999). This idea was taken up by Dethier et al. (2003) who suggested that newly arriving organisms with direct development might have advantages in colonisation since their propagules (with restricted autonomous dispersal capability) may remain close together. Species with direct development may establish in new areas, since their offspring recruit in immediate vicinity of their parents, facilitating the formation of new populations (Johannesson 1988). Peracarid crustaceans that release fully developed juveniles may form temporary demes (Thiel \& Vásquez 2000). Johnson et al. (2001) also suggested that, following initial colonisation, directly developing mollusc species may form more persistent populations than do species with pelagic larvae.

Gould (1977) and Jablonski \& Lutz (1983) (all cited in Ó Foighil 1989) suggested that planktotrophic larvae might represent a primary mode of development and that oceanic islands were possibly initially colonised by these larvae. The loss of pelagic larvae might have occurred secondarily in situ as a result of strong selection against mass mortality of larvae in the open ocean. Applying molecular biological methods, Ó Foighil \& Jozefowicz (1999), however, ruled out this scenario for Lasaea sp. in the North Atlantic. Island populations from the Azores and from Bermuda did not form the well-differentiated, island-specific clades that would have been expected for populations originating from an ancestral colonisation followed by subsequent loss of pelagic larvae. Furthermore, the unsuitability of pelagic larvae for population persistence on oceanic islands does not explain the loss of pelagic larvae at continental sites where larvae have been proven to be an efficient means of dispersal over small and medium distances (i.e., tens to hundreds of kilometres). Consequently, Ó Foighil (1989) is of the opinion that oceanic islands are colonised by species with direct development rather than by larvae and that the lack of feeding larvae in island species represents the primary developmental mode. The apparent advantages of direct development for successful rafting and subsequent colonisation are underlined by the high diversity and number of organisms with this reproductive mode (see also above). Carlquist (1966) cited the occurrence of viviparity in New Zealand geckos and of intersexes in a snake from a Brazilian island, reproductive traits not seen in congeners from continental populations. Possibly, the establishment of these reproductive traits among island species is a consequence of the rare frequency of arrival on an island.

In summary, reproductive traits play an important role in determining successful establishment of rafting organisms in new habitats. While these traits are of minor importance when rafting occurs within the geographic range of a species (where conspecifics are already present), they become crucial when rafting dispersal carries organisms to previously uninhabited regions (Figure 24).

\section{Rafting organisms as dispersal entities (propagules)}

When considering rafting as a transport mechanism, distances and frequency of dispersal events are of principal importance. The distance an organism can cover by rafting depends on its own 


\section{RAFTING OF BENTHIC MARINE ORGANISMS}

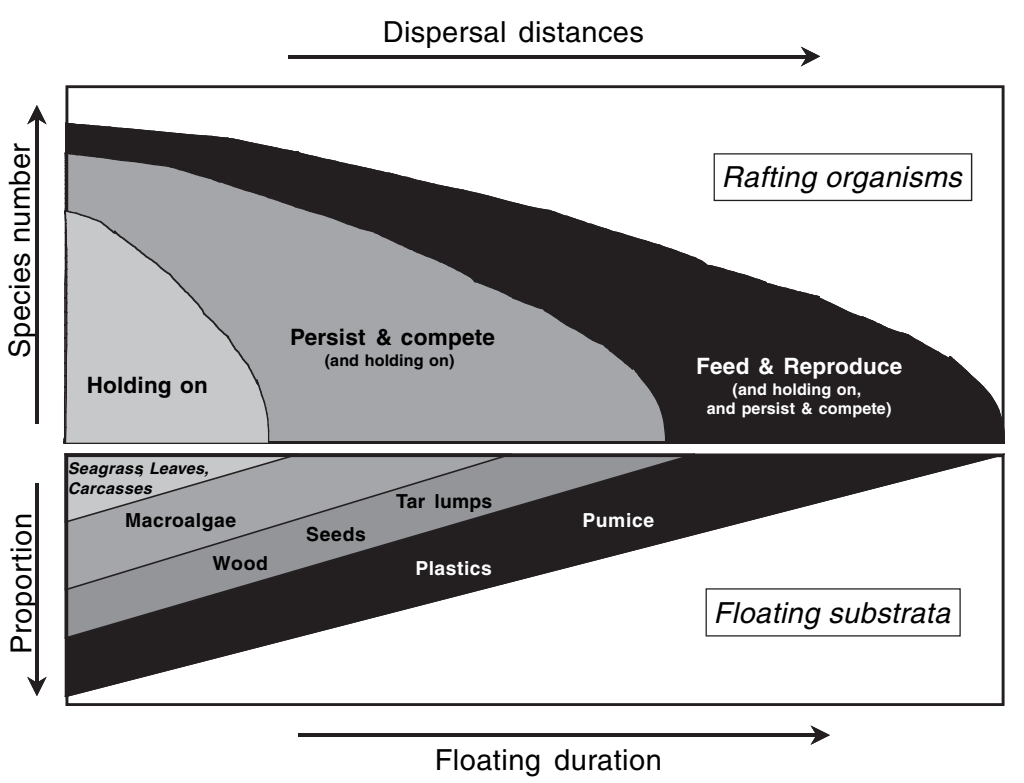

Figure 25 Relationship between substratum characteristics, floating duration (dispersal distances) and biological traits of rafting organisms. A wide variety of floating substrata has relatively short floating duration, and a large number of rafting species can be found hanging on to these, regardless of their capacity to persist and reproduce on a raft. Few floating substrata have a long floating duration and only species that combine important biological traits are found on these.

capability to persist under rafting conditions and on the longevity of its raft. In this review we focussed on the biology of organisms for which rafting has been inferred or reported, and on ecological interactions during rafting. It became evident that many organisms are well adapted to survive a rafting journey, and some species are found on floating items so frequently, and in such high densities, that rafting probably plays an important role during their life history. Possibly at certain times of the year, some of these organisms actively seek out floating items in order to achieve successful dispersal via rafting. We believe that rafting dispersal may have been a selective agent in the evolution of coastal (and some terrestrial) organisms and communities. Organisms that are well adapted to become dispersed via rafting may have selective advantages in regions and habitats with ample access to floating items. Biological traits permitting successful rafting are (1) the capacity to hold on to a floating item, (2) the capability to persist and compete successfully on a raft and (3) the potential to reproduce both during the voyage and after reaching new habitats. These traits are closely related to dispersal distances, which rafters potentially can achieve (Figure 25). In the following paragraphs, we will briefly summarise how these traits relate to dispersal potential of rafting organisms.

\section{Local dispersal (holding on)}

Any organism that can hold on to a floating item may become transported via rafting. Organisms from a wide variety of taxa appear to have the capacity to cling or attach to floating items. As was revealed in the section on species succession, this capability will be a selecting factor during initial stages of a rafting voyage. Sessile species and relatively small organisms appear to be best suited to hold on to floating items. Following this initial selection, survival of species on floating items will be intimately linked to the fate of their floating substratum. Biotic substrata such as macroalgae 


\section{MARTIN THIEL \& LARS GUTOW}

and vascular plants, which are structurally complex and offer hold to many clinging organisms, typically have shorter survival times at the sea surface than abiotic substrata such as pumice or plastics, which are relatively compact and offer less attachment sites for clinging organisms. Since both these types of floating substrata may remain afloat for time periods of 10-100 days (Thiel \& Gutow 2004), any organism that can hold on could potentially become dispersed over the distances that can be covered during this time (e.g., Edgar \& Burton 2000). Terrestrial organisms that manage to hold on but that cannot resist seawater contact will also be selected against during this phase.

Based on genetic data, Collin (2001) inferred that distances achieved by the gastropod Crepidula convexa on seagrass blades are rarely more than a few hundred metres. Field observations by Worcester (1994) on dispersal distances of seagrass-rafting ascidians supported this notion. Results by Keough \& Chernoff (1987) on rafting of the bryozoan Bugula neritina also suggested that dispersal via seagrass blades is limited to enclosed bays and distances of hundreds of metres. In sheltered bays with seagrass populations, a large number of shoots can be found floating at times. In other regions mangrove trees, saltmarsh vegetation or land plants may provide large amounts of leaves and small branches that might support short distance rafting (e.g., Wehrtmann \& Dittel 1990, Stieglitz \& Ridd 2001). Also, some small floating algae such as Colpomenia peregrina, Chaetomorpha sp., Ulva sp. or algal mats will only survive sufficiently long at the sea surface to cover distances of only a few kilometres, and rarely be transported beyond the bay of their origin. Within these bays, organisms associated with these plants or parts thereof may become dispersed via rafting over distances of several metres to kilometres. It is considered unlikely that dispersal via these floating plants occurs between bays. In all these cases, dispersal distances are primarily determined by substratum characteristics.

\section{Regional dispersal (persist and compete successfully)}

When rafting voyages last more than a few days, metabolic requirements of organisms will gain increasing importance. Species capable of surviving on stored resources, with minimal food requirements, or that can exploit food resources available in the rafting environment will be favoured. Many marine invertebrates can survive for long time periods without feeding, possibly facilitating their survival on floating items. However, these species may merely hang on without being able to grow or to reproduce. In contrast, species that can exploit available food resources can grow actively and thereby outcompete others. For example, stalked barnacles, which are obligate rafters, are optimally adapted to the rafting environment and are capable of feeding on a variety of suspended particles. They can achieve high growth rates and overgrow other rafters. Other organisms may feed on their floating substratum or on associated organisms, but since these autochthonous resources are in limited supply, they may be relatively rapidly exhausted. Small species with a low overall food intake may be favoured. Regardless of feeding rates, these secondary consumers, after having used up their resources, will be selected against during this stage of the voyage. Grazing organisms and some specialised predators may face this fate. Expression of this process may be the decreasing abundances of organisms after 20-100 days of rafting voyage (see above) and the grazer-induced destruction of floating macroalgae, which also appears to occur after about 100 days (Edgar 1987, Vásquez 1993, Hobday 2000b). It can be hypothesised that after exhaustion of autochthonous resources, only species capable of utilising allochthonous resources will persist on floating items. This suggestion is supported by the high proportion of suspension-feeders on abiotic items (see above, Winston et al. 1997, Barnes \& Sanderson 2000). These selective processes will again act strongest on terrestrial organisms, most of which cannot use food resources available on rafts. Species with dormant stages deposited on potential floating items (e.g., wood) will have a selective advantage. 


\section{RAFTING OF BENTHIC MARINE ORGANISMS}

Selection via metabolic requirements will be most important during rafting voyages extending over 10-100 days. Species persisting beyond this selective bottleneck may survive further if they are capable of competing successfully against other rafters. Based on an average rafting duration of 10 days, Hobday (2000a) assumed for floating macroalgae in southern California that "the lifetime of the raft and not the persistence time of the individual species will limit faunal dispersal". However, he also mentioned that rafts might remain afloat for up to 100 days (Hobday 2000a), and with increasing travel time, biological traits of rafters and ecological interactions will gain importance.

Several authors have inferred that intermediate dispersal distances of tens to hundreds of $\mathrm{km}$ can be achieved via large macroalgae. Edgar (1987) suggested that giant kelp Macrocystis pyrifera may be transported up to $50 \mathrm{~km}$ offshore. Hobday (2000a) estimated an average dispersal distances for this species in southern California of $56 \mathrm{~km}$. Other authors reported $M$. pyrifera and other large brown algae floating in waters hundreds of $\mathrm{km}$ from the nearest coastal source region (Safran \& Omori 1990, Helmuth et al. 1994, Ingólfsson 1995). This range of 10-1000 km can also be covered by most other floating substrata, including calcareous animal remains, wood (Donlan \& Nelson 2003), plastic items (Ye \& Andrady 1991, Winston et al. 1997) and tar balls.

\section{Long-distance dispersal (proliferate)}

With increasing duration of a rafting voyage, the reproductive biology of rafters gains importance for two reasons. Many marine invertebrates, in particular small species, have a lifetime of several months. Thus, there is an increasing probability that rafting duration exceeds the lifetime of an individual. Only species capable of proliferating during the voyage can establish persistent demes on floating items. Furthermore, with increasing duration of the voyage the probability of finding conspecifics after arrival at new coasts decreases. Species unable to proliferate, whether by asexual or sexual reproduction, will be selected against during this phase. Regardless of the mechanisms, local propagation is favoured, because it allows rafters either to recruit locally within a raft or to establish viable local populations in new habitats.

Larger distances extending beyond the regional scale $(>1000 \mathrm{~km})$ are primarily covered by persistent floating substrata such as certain wood species, seeds, volcanic pumice and plastics. Substratum characteristics are of great importance in this category and only few rafting species are able to persist over an entire oceanic voyage on these items. These species are often characterised by a sessile lifestyle, asexual reproduction, and suspension-feeding (e.g., Jokiel 1984, Winston et al. 1997).

\section{Organism biology and rafting opportunities}

During rafting dispersal the rafting assemblage is exposed to several selective bottlenecks, which gain in strength with increasing rafting duration. Any organisms capable of hanging on may survive voyages tens of days and thus become dispersed over distances in the range of tens to hundreds of kms. At this stage metabolic requirements and reproductive biology are considered to be of minor importance. Consequently, any organism, whether it produces pelagic larvae or fully developed offspring, can become dispersed via rafting over these distances. In species with pelagic larvae, though, dispersal via pelagic larvae may completely overshadow rafting dispersal. In contrast, for many species with direct development, rafting dispersal may have a pronounced effect on population dynamics. Organisms able to utilise food resources on rafts or in the rafting environment will survive rafting trips of approximately 10-100 days, permitting dispersal distances of hundreds of $\mathrm{km}$. This appears to be particularly feasible for organisms with relatively low food requirements, e.g., small marine invertebrates, and suspension-feeders. Rafting events, which may not occur very 


\section{MARTIN THIEL \& LARS GUTOW}

frequently over these distances, could result in establishment and maintenance of meta-populations of these organisms. Finally, organisms capable of local recruitment may be able to establish viable demes on rafts and new populations after rafting voyages of $>100$ days, possibly extending over $>1000 \mathrm{~km}$. Only a limited set of species will be capable of surviving these long-distance voyages, namely, species that can hang on, persist and establish local populations on rafts (Figure 25). Given the multiple selective processes that rafters may have experienced during long-lasting voyages, suspension-feeders with asexual reproduction or with direct development appear to be the most likely candidates for long-distance dispersal.

The results of this review provide support for the conceptual model developed herein (Figure 25), although it is admittedly very simplistic. Based on the presently available evidence, we have attempted to elucidate the selective processes to which rafting organisms are exposed during a rafting voyage. Furthermore we made a first intent to relate these processes and their outcome to potential dispersal distances. This model should be viewed as a first step in formulating specific hypotheses about rafting as a dispersal mechanism for marine (and terrestrial) organisms.

As pointed out in the previous review (Thiel \& Gutow 2004), successful rafting depends not only on the rafting organisms themselves but also on the quality and abundance of rafting substrata, on floating direction, and on inter- and intraspecific interactions during the journey and after debarkation. Occurrence of floating substrata may be highly variable, and periods with only a few items floating at the sea surface may alternate with periods when dense armadas of items are underway (see Thiel \& Gutow 2004). There appears to be a linkage between high abundances of floating items of terrestrial origin and intense rainfalls (see, e.g., Heatwole \& Levins 1972, ZarateVillafranco \& Ortega-García 2000) or hurricanes (Censky et al. 1998). Volkmann-Kohlmeyer \& Kohlmeyer (1993) discussed that after storms large amounts of driftwood (carrying rafting fungi) reached Hawaii. During these events many terrestrial organisms (including groups of conspecifics) may be swept to sea on large and abundant floating items (Van Duzer 2004). After volcanic eruptions, high densities of floating pumice have been reported (Coombs \& Landis 1966, Jokiel 1990b, Fushimi et al. 1991). While these events may be very rare and often irregular in occurrence, supply of floating items is high when they occur. During these events, rafting organisms are not homogeneously distributed over all floating items. Rather, many species feature a clumped distribution due to ecological interactions during the rafting journey. For example, an uneven distribution of different rafting species over available floating substrata had been observed by Ingólfsson (2000) in Icelandic waters (see also above). Highsmith (1985) also noted that rafters are not evenly distributed over floating substrata in a region but rather occur in clumped assemblages. He suggested that co-arrival of several potential colonisers should increase the probability of successful establishment.

\section{Outlook}

Rafting is a common dispersal process in present-day oceans. Coastal marine habitats harbour many species that have arrived in the past via rafting and that continue to be dispersed via rafting. Many authors pointed out that species with direct development are well suited for long-distance dispersal via rafting (e.g., Johannesson 1988, Castilla \& Guiñez 2000) and this is confirmed by the comparisons conducted herein. Species with direct development are common in most marine environments, in particular in many coastal habitats (e.g., Levin 1984, Davenport \& Stevenson 1998, Grantham et al. 2003) but also on seamounts (Parker \& Tunnicliffe 1994). The motile fauna of littoral algae is dominated by peracarid crustaceans, brooding echinoderms, bivalves and also, among the gastropods, many species that feature direct development. Interestingly, the proportion of direct developers appears to be particularly high in macroalgae known to float, often for long time periods. In some of these habitats, connectivity via rafting could thus be similarly (or more) important than exchange via pelagic larvae. 


\section{RAFTING OF BENTHIC MARINE ORGANISMS}

Based on the results of this review, several hypothesis can be formulated, e.g., small organisms are better suited for rafting dispersal than are large organisms (rationale: holding on and food requirements); substratum generalists are better suited for rafting dispersal than are substratum specialists (rationale: probability to find a raft); food generalists are better suited to survive rafting journeys than are food specialists (rationale: probability to find food); suspension feeders are better suited for long-distance rafting than are grazing species (rationale: food supply); internal fertilisers are better suited to achieve successful reproduction on rafts and in new habitats than are external fertilisers (rationale: gamete concentrations); and brooding species are better adapted to establish persistent populations on rafts and in new habitats than are species with pelagic larvae (rationale: local recruitment and future mate finding). All these hypotheses are supported by the information presented herein, but a rigorous analysis must await compilation of further data. Some of these hypotheses are difficult to test, but once additional information becomes available or when specific experiments are conducted, thorough tests of most of these hypotheses are possible. Testing of these hypotheses will foster our understanding of biological traits that favour successful rafting.

Available estimates of dispersal distances of marine organisms focus almost exclusively on species with pelagic larval stages (Kinlan \& Gaines 2003, Shanks et al. 2003, Schiel 2004), probably because for many of these species the duration of the pelagic stage is known and, in combination with predominant current speeds and directions, one can calculate approximate dispersal distances. Presently, the prevailing perception is that species with pelagic larvae depend primarily on larval dispersal, yet almost all developmental stages (except pelagic larvae) of some of these species have also been reported rafting. Rafting may in fact also be important for these species, since recent studies have shown that dispersal distances of pelagic larvae may be relatively limited, possibly because larvae exhibit behaviours retaining them near native habitats (Swearer et al. 2002).

Estimation of dispersal distances for species that rely on rafting is complicated since usually neither the duration, nor the speed, nor the direction of rafting voyages are well known. However, rafting as a dispersal mechanism provides great opportunities for researchers, because dispersal can be observed in situ. Rafting occurs at the sea surface, and floating items can be easily followed. Most rafters travel on relatively large items, which can be easily marked or deployed with transmitters that would permit tracking the rafting voyage. The usefulness of this approach has been demonstrated by studies conducted in Japan (Segawa et al. 1962) and California (Harrold \& Lisin 1989). However, to our knowledge all these studies have been conducted with floating macroalgae in coastal waters and the succession of the rafting community was not examined. We suggest that future studies should mark floating items and track them over time in order to examine the succession of the rafting community in situ (Figure 26). Deployment of transmitters on rafting items in the open ocean would also permit the detection of these items when these are approaching nearshore waters and enable the arrival process to be studied. In summary, floating items that represent an important connecting agent for rafting organisms provide ideal opportunities to study connectivity between coastal habitats. Over a local scale of hundreds of metres and following floating seagrass shoots with rafting ascidians, Worcester (1994) demonstrated the validity and feasibility of in situ observations of the rafting process. Similar studies could be done with floating macroalgae, which can be tracked directly or using radio transmitters. In particular, we consider that additional studies of the arrival and colonisation processes are necessary in order to evaluate the importance of rafting in the marine environment.

Studies of gene flow between populations of coastal marine invertebrates have often utilised a comparative approach, contrasting species with pelagic larvae with congeners that feature direct development (e.g., Russo et al. 1994, Edmands \& Potts 1997, Hoskin 1997, Chambers et al. 1998, Kyle \& Boulding 2000, Collin 2001). The hypothesis examined in these studies usually concentrated on the developing mode of the investigated species, but the results often led authors a posteriori to suggest rafting as an important and efficient dispersal mechanism for the directly developing 


\section{MARTIN THIEL \& LARS GUTOW}
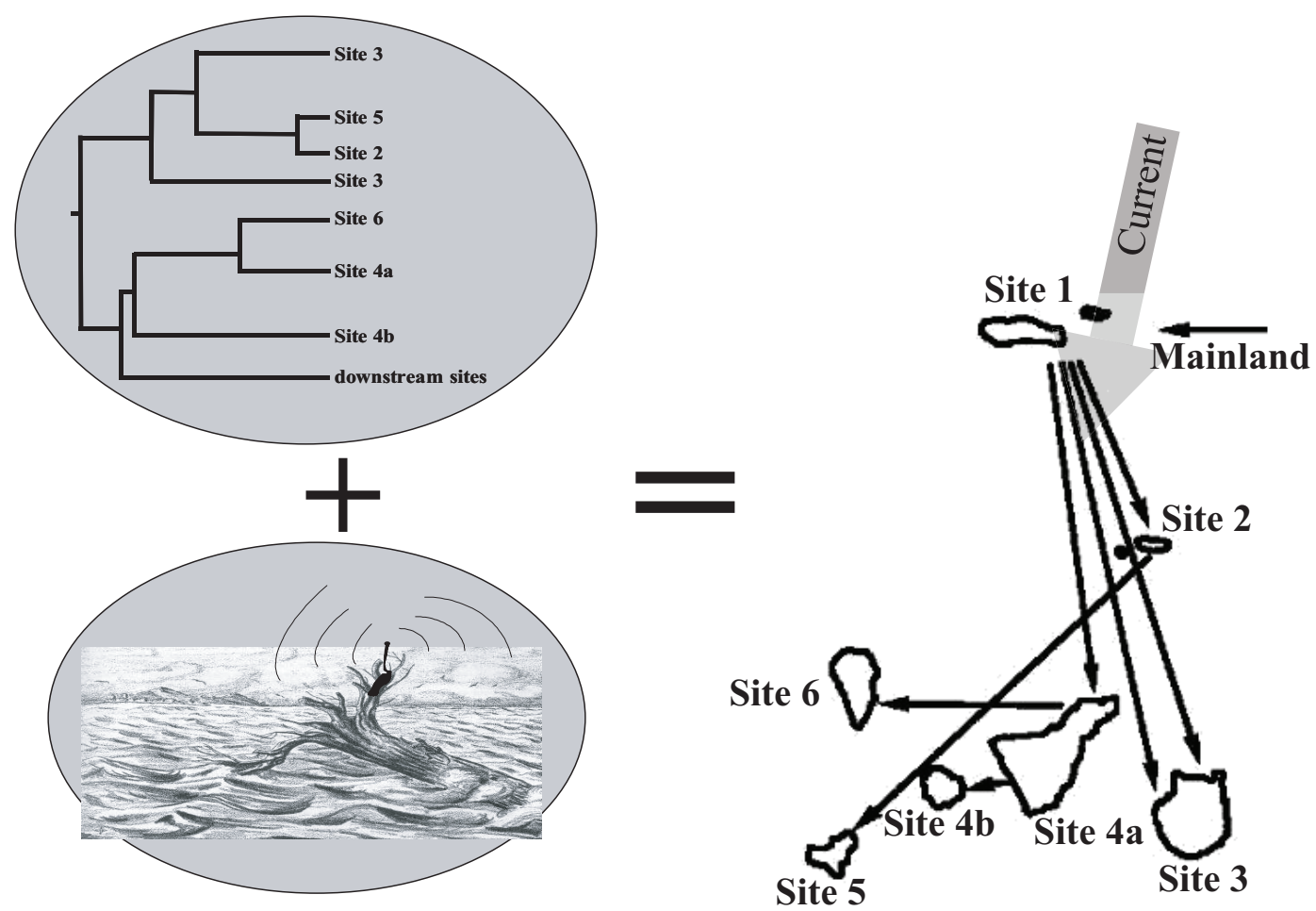

Figure 26 Future studies on the rafting process should combine genetic comparisons of species from different sites, in situ observations and repeated samplings of rafted communities and substrata (e.g., facilitated by satellite transmitters) in order to test specific hypotheses on rafting dispersal between sites. Figure adapted and modified from Carlquist (1965) and Carranza et al. (2000).

species. We suggest incorporating the rafting potential a priori in the hypotheses of future comparative studies (Figure 26). Most previous studies on gene flow have examined benthic populations separated by variable distances. Since many of those species studied can be frequently found rafting, it would also be useful to sample rafting individuals in situ. This will allow better determination of the connections between local populations and dispersal directions. Theoretical considerations suggest that meta-population models are applicable to organisms whose local populations are connected via rafting dispersal. While frequency and efficiency of rafting transport still is not well known in most marine ecosystems, for some areas knowledge appears sufficient to venture on quantitative estimates in the future. Rafting exchange between seagrass patches has been characterised on various levels, e.g., fauna and flora (Worcester 1994, Harwell \& Orth 2002) including genetic connectivity (Reusch 2002). Seagrass-dominated ecosystems with patchily distributed seagrass beds in relatively delimited bays could provide an ideal model system to examine rafting connections. In situ studies are facilitated because distances between seagrass patches rarely exceed several kilometres, and because shallow bays and relatively calm waters permit the direct tracking of floating shoots and the establishment of experimental patches. Field work should be accompanied by laboratory experiments and genetic studies. Where logistic support is available, similar studies could be conducted in open-coast kelp forests or in mangrove ecosystems. These systems are proposed for several reasons, namely because (1) they represent some of the most sensitive coastal habitats, (2) habitat patches are relatively well defined, (3) they themselves produce abundant rafting substrata and (4) they have a high retention potential for arriving rafts. 


\section{RAFTING OF BENTHIC MARINE ORGANISMS}

Such studies will also overcome one of the main drawbacks in the present knowledge of the rafting process, namely, our limited understanding of the pelago-benthic connection. It is important to concentrate on biological characteristics of rafting organisms, because connectivity between habitats depends to a high degree on the capacity of organisms to survive on rafts and to establish successfully in new habitats after arrival. Holmquist (1994) noted that organisms have a tendency to hold on to moving algae as long as these traverse inhospitable environments, further underlining that organism behaviour may influence the rafting connectivity between suitable habitats. The majority of past studies has either focussed on rafting organisms on floating items or in benthic populations. There is a strong need for integrative studies that combine sea-based and shore-based processes.

As outlined in a previous review on the availability of floating substrata as potential dispersal vehicles, rafting opportunities have changed during the past few decades due to river regulation and introduction of plastics and tar balls (Thiel \& Gutow 2004). While in some regions, species transport via floating items may have diminished as a consequence of reduced supply of floating wood, the abundance of floating plastics could have provided dispersal opportunities in other regions. Derraik (2002) emphasises the risk of species introductions as a consequence of high number of floating plastics in the world's oceans. Barnes (2002) and Barnes \& Fraser (2003) suspect that in particular the Southern Ocean might be exposed to arrival of colonisers via plastic substrata. However, tropical regions could also be susceptible to species introduction via floating plastics. Winston (1982) pointed out that the previously rare bryozoan Electra tenella is now one of the most common species on floating plastics from the Florida coast. In a subsequent extensive review, she and coauthors also mentioned other examples of potential species introductions on floating plastics in mid and low latitudes (Winston et al. 1997). It thus appears necessary to focus special attention on anthropogenic floating substrata and their role in facilitating rafting for species and in regions where rafting opportunities previously were limited.

Rafting is only one of several dispersal mechanisms in the marine environment. Dispersal via pelagic larvae (e.g., Shanks et al. 2003) and human-mediated species transport (Carlton \& Geller 1993, Ruiz et al. 2000) has received substantial research attention during the past decade. These dispersal mechanisms are of tremendous importance in coastal ecosystems since factors influencing them can have substantial consequences for population dynamics (see, e.g., studies on barnacles, bivalves, Schiel 2004, but see also Eckert 2003) or for species range expansion and invasion of previously uncolonised habitats (see, e.g., studies on crabs and bivalves, Rodríguez \& Suárez 2001, Miller et al. 2002). These effects are immediate and substantial and often are highly visible because either organisms reach enormous densities or they are relatively large (Grosholz \& Ruiz 2003). In contrast, species that are commonly dispersed via rafting are comparatively small and have a limited reproductive potential (see above). Consequently, rafting introductions may go unnoticed for long time periods and buildup of extensive populations may proceed relatively slowly if at all. Due to this, rafting dispersal may not have as obvious and immediate effects on coastal marine communities as dispersal via pelagic larvae or via human activities. Yet, rafting dispersal may nevertheless happen frequently and in the long term have a profound effect on the biodiversity of coastal communities. Even though many studies have hinted towards and are continuing to indicate the importance of rafting, it remains one of the most enigmatic dispersal mechanisms in the oceans.

\section{Acknowledgements}

Numerous colleagues provided literature and important information and we are extremely grateful to all of them. This review also benefited from the translations of Japanese and Russian literature, for which we thank Hiroshi Kajihara and Sergey Dobretsov. We are especially thankful to Erasmo Macaya who diligently helped with the preparation of the figures. The members of the BEDIM 


\section{MARTIN THIEL \& LARS GUTOW}

laboratory provided critical comments to a first draft. Inken Kruse and Christian Buschbaum carefully read the entire manuscript and helped bring it in a presentable shape. MT acknowledges an invitation by the Alfred Wegener Institute for Polar and Marine Research, which greatly facilitated the preparation of this manuscript. We thank P. Haye for some last-minute comments that helped us to improve some of the conceptual figures. Financial support was provided through FONDECYT 1010356 to MT. We thank P. Haye for some last-minute comments that helped us to improve some of the conceptual figures.

\section{References}

Abe, T. 1984. Colonization of the Krakatau Islands by termites (Insecta: Isoptera). Physiology and Ecology Japan 21, 63-88.

Adachi, N. \& Wada, K. 1999. Distribution in relation to life history in the direct-developing gastropod Batillaria cumingi (Batillariidae) on two shores of contrasting substrata. Journal of Molluscan Studies 65, 275-288.

Adams, J.A. 1960. A contribution to the biology and postlarval development of the Sargassum fish, Histrio histrio (Linnaeus), with a discussion of the Sargassum complex. Bulletin of Marine Science of the Gulf and Caribbean 10, 55-82.

Albrecht, A. \& Reise, K. 1994. Effects of Fucus vesiculosus covering intertidal mussel beds in the Wadden Sea. Helgoländer Meeresuntersuchungen 48, 243-256.

Aliani, S. \& Molcard, A. 2003. Hitch-hiking on floating marine debris: macrobenthic species in the Western Mediterranean Sea. Hydrobiologia 503, 59-67.

Andersen, N.M. \& Cheng, L. 2004. The marine insect Halobates (Heteroptera: Gerridae): biology, adaptations, distribution, and phylogeny. Oceanography and Marine Biology: An Annual Review 42, 119-154.

Anger, K. 2001. The Biology of Decapod Crustacean Larvae. Lisse: A.A. Balkema Publishers.

Anraku, M. \& Azeta, M. 1967. The feeding activities of yellowtail larvae, Seriola quinqueradiata Temminck et Schlegel, associated with floating seaweeds. Bulletin of the Seikai National Fisheries Research Institute, Nagasaki 35, 41-50.

Anthony, K.R.N. \& Svane, I. 1995. Effects of substratum instability on locomotion and pedal laceration in Metridium senile (Anthozoa: Actiniaria). Marine Ecology Progress Series 124, 171-180.

Aoki, M. \& Kikuchi, T. 1995. Notes on Caprella andreae Mayer, 1890 (Crustacea: Amphipoda) from the carapace of Loggerhead Sea Turtles in the East China Sea and in Kyushu, Japan. Proceedings of the Japan Society of Systematic Zoology 53, 54-61.

Armonies, W. \& Hartke, D. 1995. Floating of mud snails Hydrobia ulvae in tidal waters of the Wadden Sea, and its implications in distribution patterns. Helgoländer Meeresuntersuchungen 49, 529-538.

Arnaud, F., Arnaud, P.M., Intes, A. \& Loeuff, P. 1976. Transport d'invertébrés benthiques entre l'Afrique du Sud et Saint Hélène par les laminaires (Phaeophyceae). Bulletin du Museum National d'Histoire Naturelle Paris Séries 3 384, 49-55.

Arnaud, P.M. 1973. Le genre Lepas Linné, 1767 dans les Terres Australes et Antarctiques Francaises (Crustacea Cirripedia). Crustaceana 23, 157-162.

Arnaud, P.M. 1974. Contribution à la bionomie marine benthique des régions antarctiques et sub-antarctiques. Téthys 3, 465-656.

Averincev, V.G. 1980. The role of drifting islands of seaweeds in the distribution of bottom polychaetes. Biologiya Morya, Vladivostok 1, 80-83.

Azam, F. \& Long, R.A. 2001. Oceanography: sea snow microcosms. Nature 414, 495-498.

Barbour, T. 1916. Some remarks upon Matthew's "Climate and Evolution”. Annals of the New York Academy of Sciences 27, 1-10.

Barnes, D.K.A. 2002. Biodiversity: invasions by marine life on plastic debris. Nature 416, 808-809.

Barnes, D.K.A. \& Fraser, K.P.P. 2003. Rafting by five phyla on man-made flotsam in the Southern Ocean. Marine Ecology Progress Series 262, 289-291.

Barnes, D.K.A. \& Sanderson, W.G. 2000. Latitudinal patters in the colonization of marine debris. In Proceedings of the 11th International Bryozoology Association Conference, A. Herrera Cubilla \& J.B.C. Jackson (eds). Balboa: Smithsonian Tropical Research Institute, 145-153. 


\section{RAFTING OF BENTHIC MARINE ORGANISMS}

Bayly, I.A.E., Gibson, J.A.E., Wagner, B. \& Swadling, K.M. 2003. Taxonomy, ecology and zoogeography of two East Antarctic freshwater calanoid copepod species: Boeckella poppei and Gladioferens antarcticus. Antarctic Science 15, 439-448.

Benham, W.B. 1902. The geographical distribution of earthworms and the palaeography of the Antarctic region. Report of the Australasian Association of Advanced Science 9.

Bers, A.V. \& Wahl, M. 2004. The influence of natural surface microtopographies on fouling. Biofouling 20, 43-51.

Besada, E.G., Loeblich, L.A. \& Loeblich III, A.R., 1982. Observations on tropical, benthic dinoflagellates from Ciguatera-endemic areas: Coolia, Gambierdiscus, and Ostreopsis. Bulletin of Marine Science 32, 723-735.

Beukema, J.J. \& Vlas, J. 1989. Tidal-current transport of thread-drifting postlarval juveniles of the bivalve Macoma balthica from the Wadden Sea to the North Sea. Marine Ecology Progress Series 52, 193-200.

Bieler, R. 1995. Mathildidae from New Caledonia and the Loyalty Islands (Gastropoda: Heterobranchia). Memoires du Museum National d'Histoire naturelle, Paris (France) 167, 595-641.

Bingham, B.L. 1992. Life histories in an epifaunal community: coupling of adult and larval processes. Ecology 73, 2244-2259.

Bingham, B.L. \& Young, C.M. 1995. Stochastic events and dynamics of a mangrove root epifaunal community. Pubblicazioni della Stazione Zoologica di Napoli I Marine Ecology 16, 145-163.

Birkhead, T.R. \& Moller, A.P. 1993. Sexual selection and the temporal separation of reproductive events: sperm storage data from reptiles, birds and mammals. Biological Journal of the Linnean Society 50, 295-311.

Bomber, J.W., Morton, S.L., Babinchak, J.A., Norris, D.R. \& Morton, J.G. 1988. Epiphytic dinoflagellates of drift algae - another toxigenic community in the ciguatera food chain. Bulletin of Marine Science 43, 204-214.

Bonardelli, J., Himmelman, J.H. \& Drinkwater, K. 1996. Relation of spawning of the giant scallop, Placopecten magellanicus, to temperature fluctuations during downwelling events. Marine Biology 124, 637-649.

Borges, L.M.S., Cragg, S.M. \& Williams, J.R. 2003. Comparing the resistance of a number of lesser known species of tropical hardwoods to the marine borer Limnoria using a short term laboratory assay. International Research Group on Wood Preservation, Document No. IRG/WP 03-10500.

Brandon-Jones, D. 1998. Pre-glacial Bornean primate impoverishment and Wallace's line. In Biogeography and Geological Evolution of SE Asia, R. Hall \& J.D. Holloway (eds). Leiden: Backhuys Publishers, 393-404.

Brooks, R.A. 2004. Discovery of Sphaeroma terebrans, a wood-boring isopod, in the red mangrove, Rhizophora mangle, habitat of northern Florida Bay. Ambio 33, 171-173.

Brooks, R.A. \& Bell, S.S. 2001a. Colonization of a dynamic substrate: factors influencing recruitment of the wood-boring isopod, Sphaeroma terebrans, onto red mangrove (Rhizophora mangle) prop roots. Oecologia 127, 522-532.

Brooks, R.A. \& Bell, S.S. 2001b. Mobile corridors in marine landscapes: enhancement of faunal exchange at seagrass/sand ecotones. Journal of Experimental Marine Biology and Ecology 264, 67-84.

Brusca, R.C. \& Brusca, G.J. 2003. Invertebrates. Sunderland, Massachusetts: Sinauer Associates.

Burns, K.A. \& Teal, J.M. 1973. Hydrocarbons in the pelagic Sargassum community. Deep-Sea Research Oceanographic Abstracts 20, 207-211.

Buroker, N.E., Chanley, P., Cranfield, H.J. \& Dinamani, P. 1983. Systematic status of two oyster populations of the genus Tiostrea from New Zealand and Chile. Marine Biology 77, 191-200.

Buschmann, A.H. 1990. Intertidal macroalgae as refuge and food for Amphipoda in central Chile. Aquatic Botany 36, 237-245.

Bushing, W.W. 1994. Biogeographic and ecological implications of kelp rafting as a dispersal vector for marine invertebrates. In Proceedings of the Fourth California Islands Symposium: Update on the Status of Resources, March 22-25, 1994, W.L. Halvorson \& G.J. Maender (eds). Santa Barbara, California: Santa Barbara Museum of Natural History, 103-110.

Butler, J.N. 1975. Pelagic tar. Scientific American 232, 90-97.

Butler, J.N., Morris, B.F., Cadwallader, J. \& Stoner, A.W. 1983. Studies of Sargassum and the Sargassum community. Bermuda Biological Station Special Publications 22, 1-307. 


\section{MARTIN THIEL \& LARS GUTOW}

Byrne, M. 1995. Changes in larval morphology in the evolution of benthic development by Patiriella exigua (Asteroidea: Asterinidae), a comparison with the larvae of Patiriella species with planktonic development. Biological Bulletin (Woods Hole) 188, 293-305.

Cadée, G.C. 1999. Eikapsels van de fuikhoorn Nassarius reticulatus aangespoeld op Texel. Het Zeepard 59, 4-9.

Cadée, G.C. 2002. Mass stranding of cuttlebones of Sepia orbignyana Férussac, 1826, on Texel, the Netherlands, in July 2002 (Cephalopoda, Decapoda, Sepiidae). Basteria 66, 113-120.

Calder, D.R. 1993. Local distribution and biogeography of the hydroids (Cnidaria) of Bermuda. Caribbean Journal of Science 29, 61-74.

Calder, D.R. 1995. Hydroid assemblages on holopelagic Sargassum from the Sargasso Sea at Bermuda. Bulletin of Marine Science 56, 537-546.

Caldwell, D.K. 1969. Baby loggerhead turtles associated with Sargassum weed. Quarterly Journal of the Florida Academy of Science 31, 271-272.

Calsbeek, R. \& Smith, T.B. 2003. Ocean currents mediate evolution in island lizards. Nature 426, 552-555.

Capuzzo, J.M. 1987. Biological effects of petroleum hydrocarbons: assessments from experimental results. In Long-Term Environmental Effects of Offshore Oil and Gas Development, D.F. Boesch \& N.N. Rabalais (eds). London: Elsevier Applied Science, 343-410.

Carlquist, S. 1965. Island Life - A Natural History of the Islands of the World. Garden City, New York: The Natural History Press.

Carlquist, S. 1966. The biota of long distance dispersal. I. Principles of dispersal and evolution. Quarterly Journal of Biology 41, 247-270.

Carlquist, S. 1967. The biota of long distance dispersal. V. Plant dispersal to the Pacific Islands. Bulletin of the Torrey Botanical Club 94, 129-162.

Carlton, J.T. 2003. Community assembly and historical biogeography in the North Atlantic Ocean: the potential role of human-mediated dispersal vectors. Hydrobiologia 503, 1-8.

Carlton, J.T. \& Geller, J.B. 1993. Ecological roulette: the global transport of nonindigenous marine organisms. Science 261, 78-82.

Carpenter, E.J. 1970. Diatoms attached to floating Sargassum in the western Sargasso Sea. Phycologia 9 , 271-274.

Carpenter, E.J. 1972. Nitrogen fixation by a blue-green epiphyte on pelagic Sargassum. Science 178, 1207-1209.

Carpenter, E.J. \& Cox, J.L. 1974. Production of pelagic Sargassum and a blue-green epiphyte in the western Sargasso Sea. Limnology and Oceanography 19, 429-436.

Carpenter, E.J. \& Smith, K.L. 1972. Plastics on the Sargasso Sea surface. Science 175, 1240-1241.

Carr, A.F. \& Meylan, A.B. 1980. Evidence of passive migration of green turtle hatchlings in Sargassum. Copeia 1980, 366-368.

Carranza, S. \& Arnold, E.N. 2003. Investigating the origin of transoceanic distributions: mtDNA shows Mabuya lizards (Reptilia, Scincidae) crossed the Atlantic twice. Systematics and Biodiversity 1, 275-282.

Carranza, S., Arnold, E.N., Mateo, J.A. \& Lopez-Jurado, L.F. 2000. Long-distance colonization and radiation in gekkonid lizards, Tarentola (Reptilia: Gekkonidae), revealed by mitochondrial DNA sequences. Proceedings of the Royal Society of London Series B 267, 637-649.

Castilla, J.C. \& Guiñez, R. 2000. Disjoint geographical distribution of intertidal and nearshore benthic invertebrates in the southern hemisphere. Revista Chilena De Historia Natural 73, 585-603.

Castro, J.J., Santiago, J.A. \& Santana-Ortega, A.T. 2002. A general theory on fish aggregation to floating objects: an alternative to the meeting point hypothesis. Reviews in Fish Biology and Fisheries 11, 255-277.

Censky, E.J., Hodge, K. \& Dudley, J. 1998. Over-water dispersal of lizards due to hurricanes. Nature 395, 556.

Chace, F.A. 1951. The oceanic crabs of the genera Planes and Pachygrapsus. Proceedings of the United States National Museum 101, 65-103.

Chace, F.A. 1966. Decapod crustaceans from St. Helena Island, South Atlantic. Proceedings of the United States National Museum 118, 623-660.

Chamberlain Jr, J.A., Ward, P.D. \& Scott Weaver, J. 1981. Post-mortem ascent of Nautilus shells: Implications for cephalopod paleobiogeography. Paleobiology 4, 494-509. 


\section{RAFTING OF BENTHIC MARINE ORGANISMS}

Chambers, R.J., McQuaid, C.D. \& Kirby, R. 1998. The use of randomly amplified polymorphic DNA to analyze the genetic diversity, the systematic relationships and the evolution of intertidal limpets, Siphonaria spp. (Pulmonata: Gastropoda), with different reproductive modes. Journal of Experimental Marine Biology and Ecology 227, 49-66.

Chaparro, O.R., Saldivia, C.L. \& Paschke, K.A. 2001. Regulatory aspects of the brood capacity of Crepidula fecunda Gallardo 1979 (Gastropoda: Calyptraeidae). Journal of Experimental Marine Biology and Ecology 266, 97-108.

Cheng, L. 1975. Marine pleuston — animals at the sea-air interface. Oceanography and Marine Biology: An Annual Review 13, 181-212.

Cheng, L. \& Birch, M.C. 1977. Terrestrial insects at sea. Journal of the Marine Biological Association of the United Kingdom 57, 995-997.

Cheng, L. \& Lewin, R.A. 1975. Flatworms afloat. Nature 258, 518-519.

Chess, J.R. 1993. Effects of the stipe-boring amphipod Peramphithoe stypotrupetes (Corophioidea: Ampithoidae) and grazing gastropods on the kelp Laminaria setchellii. Journal of Crustacean Biology 13, 638-646.

Chirat, R. 2000. The so-called 'cosmopolitan palaeobiogeographic distribution' of Tertiary Nautilida of the genus Aturia Bronn 1838: the result of post-mortem transport by oceanic palaeocurrents. Palaeogeography, Palaeoclimatology, Palaeoecology 157, 59-77.

Cho, S.H., Myoung, J.G., Kim, J.M. \& Lee, J.H. 2001. Fish fauna associated with drifting seaweed in the coastal area of Tongyeong, Korea. Transactions of the American Fisheries Society 130, 1190-1202.

Clench, W.J. 1925. A possible manner of snake distribution. Copeia 1925, 40.

Collin, R. 2001. The effects of mode of development on phylogeography and population structure of North Atlantic Crepidula (Gastropoda: Calyptraeidae). Molecular Ecology 10, 2249-2262.

Commitee on Oil in the Sea: Inputs, Fates, and Effects, Ocean Studies Board and Marine Board, Divisions of Earth and Life Studies and Transportation Research Board, National Research Council 2003. Oil in the Sea III. Washington, D.C.: The National Academic Press.

Conover, J.T. \& Sieburth, J.M. 1964. Effect of Sargassum distribution on its epibiota and antibacterial activity. Botanica Marina 6, 147-157.

Coombs, D.S. \& Landis, C.S. 1966. Pumice from the South Sandwich eruption of March 1962 reaches New Zealand. Nature 209, 289-290.

Cornelius, P.F.S. 1992a. The Azores hydroid fauna and its origin, with discussion of rafting and medusa suppression. Arquipelago 10, 75-99.

Cornelius, P.F.S. 1992b. Medusa loss in leptolid Hydrozoa (Cnidaria), hydroid rafting, and abbreviated lifecycles among their remote-island faunae: an interim review. Scientia Marina 56, 245-261.

Coston-Clements, L., Dettle, L.R., Hoss, D.E. \& Cross, F.A. 1991. Utilization of the Sargassum habitat by marine invertebrates and vertebrates - a review. NOAA Technical Memorandum NMFS-SEFSC-296.

Coulson, S.J., Hodkinson, I.D., Webb, N.R. \& Harrison, J.A. 2002. Survival of terrestrial soil-dwelling arthropods on and in seawater: implications for trans-oceanic dispersal. Functional Ecology 16, 353-356.

Coyer, J.A., Smith, G.J. \& Andersen, R.A. 2001. Evolution of Macrocystis spp. (Phaeophyceae) as determined by ITS1 and ITS2 sequences. Journal of Phycology 37, 574-585.

Crossland, C. 1952. Madreporaria, Hydrocorallinae, Heliopora and Tubipora. Scientific Reports: Great Barrier Reef Expedition 1928-29 6, 86-257.

Crozier, W.J. 1918. Note on the coloration of Planes minutus. American Naturalist 52, 616-617.

Culliney, J.L. 1970. Measurements of reactive phosphorus associated with pelagic Sargassum in the northwest Sargasso Sea. Limnology and Oceanography 15, 304-306.

Cundell, A.M., Sleeter, T.D. \& Mitchell, R. 1978. Microbial populations associated with the surface of the brown alga Ascophyllum nodosum. Microbial Ecology 4, 81-91.

Cunningham, C.W. \& Collins, T.M. 1998. Beyond area relationships: extinction and recolonization in molecular marine biogeography. In Molecular Approaches to Ecology and Evolution, R. DeSalle \& B. Schierwater (eds). Basel: Birkhäuser Verlag, 297-321.

Daborn, G.R. \& Gregory, R.S. 1983. Occurrence, distribution, and feeding habits of juvenile lumpfish, Cyclopterus lumpus L. in the Bay of Fundy. Canadian Journal of Zoology 61, 797-801. 


\section{MARTIN THIEL \& LARS GUTOW}

Dahl, A.L. 1984. Biogeographical aspects of isolation in the Pacific. Ambio 13, 302-305.

Daniel, G., Nilsson, T. \& Cragg, S.M. 1991. Limnoria lignorum ingest bacterial and fungal degraded wood. Holz als Roh- und Werkstoff 49, 488-490.

Davenport, J. 1992. Observations on the ecology, behaviour, swimming mechanism and energetics of the neustonic grapsid crab, Planes minutus. Journal of the Marine Biological Association of the United Kingdom 72, 611-620.

Davenport, J. \& Rees, E.I.S. 1993. Observations on neuston and floating weed patches in the Irish Sea. Estuarine Coastal and Shelf Science 36, 395-411.

Davenport, J. \& Stevenson, T.D.I. 1998. Intertidal colonization rates. A matched latitude, north v. south, remote v. near shore island experiment. Diversity and Distributions 4, 87-92.

Delgadillo, C. 2000. Mosses and the Caribbean connection between North and South America. Bryologist 103, 82-86.

Dell, R.K. 1964. The oceanic crab, Pachygrapsus marinus (Rathbun) in the south-west Pacific. Crustaceana 7, 79-80.

Dell, R.K. 1972. Antarctic benthos. Advances in Marine Biology 10, 1-216.

Dellinger, T., Davenport, J. \& Wirtz, P. 1997. Comparisons of social structure of Columbus crabs living on loggerhead sea turtles and inanimate flotsam. Journal of the Marine Biological Association of the United Kingdom. Plymouth 77, 185-194.

De Matthaeis, E., Davolos, D. \& Cobolli, M. 1998. Genetic divergence between populations and species of talitrids from Aegean Islands. Journal of Heredity 89, 37-43.

De Matthaeis, E., Davolos, D., Cobolli, M. \& Ketmaier, V. 2000. Isolation by distance in equilibrium and nonequilibrium populations of four talitrid species in the Mediterranean Sea. Evolution 54, 1606-1613.

Derraik, J.G.B. 2002. The pollution of the marine environment by plastic debris: a review. Marine Pollution Bulletin 44, 842-852.

Dethier, M.N., McDonald, K. \& Strathmann, R.R. 2003. Colonization and connectivity of habitat patches for coastal marine species distant from source populations. Conservation Biology 17, 1024-1035.

Deudero, S. \& Morales-Nin, B. 2000. Occurrence of Polyprion americanus under floating objects in western Mediterranean oceanic waters, inference from stomach contents analysis. Journal of the Marine Biological Association of the United Kingdom 80, 751-752.

Deudero, S. \& Morales-Nin, B. 2001a. Surface mesozooplankton in open waters of the Western Mediterranean. Ophelia 54, 1-13.

Deudero, S. \& Morales-Nin, B. 2001b. Prey selectivity in planktivorous juvenile fishes associated with floating objects in the western Mediterranean. Aquaculture Research 32, 481-490.

DeVantier, L.M. 1992. Rafting of tropical marine organisms on buoyant coralla. Marine Ecology Progress Series 86, 301-302.

Deysher, L. \& Norton, T. A. 1981. Dispersal and colonization in Sargassum muticum (Yendo) Fensholt. Journal of Experimental Marine Biology and Ecology 56, 179-195.

Dixon, J., Schroeter, S.C. \& Kastendiek, J. 1981. Effects of the encrusting bryozoan, Membranipora membranacea, on the loss of blades and fronds by the giant kelp, Macrocystis pyrifera (Laminariales). Journal of Phycology 17, 341-345.

Docters van Leeuwen, W.M. 1936. Krakatau 1883-1933. A. Botany. Annales du Jardin Botanique de Buitenzorg 46-47, 1-507.

Domaneschi, O., da Silva, J., Neto, L. \& Passos, F. 2002. New perspectives on the dispersal mechanisms of the Antarctic brooding bivalve Mysella charcoti (Lamy, 1906). Polar Biology 25, 538-541.

Donlan, C.J. \& Nelson, P.A. 2003. Observations of invertebrate colonized floatsam in the eastern tropical Pacific, with a discussion of rafting. Bulletin of Marine Science 72, 231-240.

Dooley, J.K. 1972. Fishes associated with the pelagic Sargassum complex, with a discussion of the Sargassum community. Contributions in Marine Science 16, 1-32.

Dow, T.G. \& Menzies, R.J. 1958. The pelagic isopod Idotea metallica in the Mediterranean. Pubblicazioni della Stazione Zoologica di Napoli 30, 330-336.

Druce, B.E. \& Kingsford, M.J. 1995. An experimental investigation on the fishes associated with drifting objects in coastal waters of temperate Australia. Bulletin of Marine Science 57, 378-392. 


\section{RAFTING OF BENTHIC MARINE ORGANISMS}

Dudgeon, S., Kubler, J.E., Wright, W.A., Vadas, R.L. \& Petriatis, P.S. 2001. Natural variability in zygote dispersal of Ascophyllum nodosum at small spatial scale. Functional Ecology 15, 595-604.

Duke, N.C. 1995. Genetic diversity, distributional barriers and rafting continents - more thoughts on the evolution of mangroves. Hydrobiologia 295, 167-181.

Dyke, A.S., England, J., Reimnitz, E. \& Jette, H. 1997. Changes in driftwood delivery to the Canadian Arctic Archipelago: the hypothesis of postglacial oscillations of the transpolar drift. Arctic 50, 1-16.

Ebert, T.A. 1996. The consequence of broadcasting, brooding, and asexual reproduction in echinoderm metapopulations. Oceanologica Acta 19, 217-226.

Eckert, G.L. 2003. Effects of the planktonic period on marine population fluctuations. Ecology 84, 372-383.

Edgar, G.J. 1987. Dispersal of faunal and floral propagules associated with drifting Macrocystis pyrifera plants. Marine Biology 95, 599-610.

Edgar, G.J. 1992. Patterns of colonization of mobile epifauna in a Western Australian seagrass bed. Journal of Experimental Marine Biology and Ecology 157, 225-246.

Edgar, G.J. \& Burton, H.R. 2000. The biogeography of shallow-water macrofauna at Heard Island. Papers and Proceedings of the Royal Society of Tasmania 133, 23-26.

Edmands, S. \& Potts, D.C. 1997. Population genetic structure in brooding sea anemones (Epiactis spp.) with contrasting reproductive modes. Marine Biology 127, 485-498.

Edmondson, C.H. 1946. Dispersal of shipworms among Central Pacific Islands, with description of new species. Occasional Papers of Bernice P. Bishop Museum, Honolulu, Hawaii 18, 211-224.

Edmondson, C.H. 1962. Teredinidae, ocean travelers. Occasional Papers of Bernice P. Bishop Museum, Honolulu, Hawaii 23, 45-59.

Edwards, S.F. \& Welsh, B.L. 1982. Trophic dynamics of a mud snail [Ilyanassa obsoleta (Say)] population on an intertidal mudflat. Estuarine Coastal and Shelf Science 14, 663-686.

Ekman, S. 1967. Zoogeography of the Sea. London: Sidgwick \& Jackson.

El-Shanshoury, A.R., Mona, M.H., Shoukr, F.A. \& El-Bossery, A.M. 1994. The enumeration and characterization of bacteria and fungi associated with marine wood-boring isopods, and the ability of these microorganisms to digest cellulose and wood. Marine Biology 119, 321-326.

Emery, K.O. 1955. Transportation of rocks by driftwood. Journal of Sedimentary Petrology 25, 51-57.

Emery, K.O. 1963. Organic transportation of marine sediments. In The Sea, M.N. Hill (ed.). New York: Wiley, 776-793.

Evans, F. 1958. Growth and maturity of the barnacles Lepashillii and Lepas anatifera. Nature 182, 1245-1246.

Fage, L. 1949. Classe des Pycnogonides. In Traité de Zoologie, P.-P. Grassé (ed.). Paris: Masson et Cie., 906-941.

Fairbridge, R.W. \& Teichert, C. 1948. The Low Isles of the Great Barrier Reef: a new analysis. The Geographical Journal 111, 67-88.

Faust, M.A. \& Gulledge, R.A. 1996. Associations of microalgae and meiofauna in floating detritus at a mangrove island, Twin Cays, Belize. Journal of Experimental Marine Biology and Ecology 197, 159-175.

Fedoryako, B.I. 1989. A comparative characteristic of oceanic fish assemblages associated with floating debris. Journal of Ichthyology 29, 128-137.

Feilden, H.W. 1889. Notes on the reptiles of Barbados. Zoologist, Ser. 3, 13, 295-298.

Fell, H.-B. 1953. Echinoderms from the subantarctic islands of New Zealand: Asteroidea, Ophiuroidea, and Echinoidea. Cape Expedition Series, Scientific Results of the New Zealand Subantarctic Expedition, 1941-1945. Dominion Museum Records in Zoology (Wellington) 2, 73-111.

Fell, H.B. 1962. West-wind drift dispersal of echinoderms in the Southern Hemisphere. Nature 193, 759-761.

Fell, H.B. 1967. Cretaceous and Tertiary surface currents of the oceans. Oceanography and Marine Biology: An Annual Review 5, 317-347.

Fine, M.L. 1970. Faunal variation on pelagic Sargassum. Marine Biology 7, 112-122.

Fletemeyer, J.R. 1978. Underwater tracking of neonate loggerhead seaturtles seeking shelter in drifting Sargassum. Copeia 1978, 148-149.

Fox, W. 1963. Special tubules for sperm storage in female lizards. Nature 198, 500-501.

Franke, H.D. 1993. Mating system of the commensal marine isopod Jaera hopeana (Crustacea) I. The malemanca(I) amplexus. Marine Biology 115, 65-73. 


\section{MARTIN THIEL \& LARS GUTOW}

Franke, H.-D. \& Gutow, L. 2004. Long-term changes in the macro-zoobenthos around the rocky island of Helgoland (German Bight, North Sea). Helgoland Marine Research 58, 303-310.

Franke, H.-D., Gutow, L. \& Janke, M. 1999. The recent arrival of the oceanic isopod Idotea metallica Bosc off Helgoland (German Bight, North Sea): an indication of a warming trend in the North Sea? Helgoländer Meeresuntersuchungen 52, 347-357.

Franke, H.-D. \& Janke, M. 1998. Mechanisms and consequences of intra- and interspecific interference competition in Idotea baltica (Pallas) and Idotea emarginata (Fabricius) (Crustacea: Isopoda): a laboratory study of possible proximate causes of habitat segregation. Journal of Experimental Marine Biology and Ecology 227, 1-21.

Friedrich, H. 1969. Marine Biology. Seattle: University of Washington Press.

Fushimi, K., Nishikawa, M. \& Mitsuda, H. 1991. Floating pumices in the western North Pacific. The Oceanographical Magazine/Kishocho Obun Kaiyo Hokoku 41, 59-74.

Gallager, S.M., Turner, R.D. \& Berg Jr, C.J., 1981. Physiological aspects of wood consumption, growth, and reproduction in the shipworm Lyrodus pedicellatus Quatrefages (Bivalvia: Teredinidae). Journal of Experimental Marine Biology and Ecology 52, 63-77.

Gathorne-Hardy, F.J. \& Jones, D.T. 2000. The recolonization of the Krakatau islands by termites (Isoptera), and their biogeographical origins. Biological Journal of the Linnean Society 71, 251-267.

Gebauer, P., Paschke, K. \& Anger, K. 2003. Delayed metamorphosis in decapod crustaceans: evidence and consequences. Revista Chilena De Historia Natural 76, 169-175.

Gee, J.M. 1963. Pelagic life of Spirorbis larvae. Nature 198, 1109-1110.

Geiselmann, J. 1983. The food web of the Sargassum community. Bermuda Biological Station Special Publications 22, 260-270.

Gerlach, S.A. 1977. Means of meiofaunal dispersal. Mikrofauna Meeresboden 61, 89-103.

Gerrodette, T. 1981. Dispersal of the solitary coral Balanophyllia elegans by demersal planular larvae. Ecology 62, 611-619.

Giese, A.C. \& Pearse, J.S. 1977. Reproduction of Marine Invertebrates - Volume 4: Mollusc: Gastropods and Cephalopods. New York: Academic Press.

Glynn, P.W. \& Ault, J.S. 2000. A biogeographic analysis and review of the far eastern Pacific coral reef region. Coral Reefs 19, 1-23.

González, S.A. \& Edding, M.E. 1990a. Extension of the range of Heterozostera tasmanica (Martens ex Aschers.) den Hartog in Chile. Aquatic Botany 38, 391-395.

González, S.A. \& Edding, M.E. 1990b. Achelia assimilis (Haswell, 1884) in the Heterozostera bed of Puerto Aldea, Coquimbo: first record from the northern Chilean coast (Pycnogonida: Ammotheidae). Proceedings of the Biological Society of Washington 103, 151-156.

Gooding, R.M. \& Magnuson, J.J. 1967. Ecological significance of a drifting object to pelagic fishes. Pacific Science 21, 486-497.

Goodsir, H.D.S. 1845. Description of some animals found amongst the gulf-weed. The Annals and Magazine of Natural History Zoology Botany and Geology 15, 73-76.

Gorelova, T.A. \& Fedoryako, B.I. 1986. Topic and trophic relations in fishes associated with floating gulfweed biocoenosis. Voprosy Ikhtiologii 26, 94-102.

Gould, S.J. 1977. Ontogeny and Phylogeny. Cambridge: Harvard University Press.

Grantham, B.A., Eckert, G.L. \& Shanks, A.L. 2003. Dispersal potential of marine invertebrates in diverse habitats. Ecological Applications 13, S108-S116.

Gravier-Bonnet, N. 1992. Cloning and dispersal by buoyant autotomised hydranths of a thecate hydroid (Cnidaria; Hydrozoa). Scientia Marina 56, 229-236.

Green, A., Tyler, P.A., Angel, M.V. \& Gage, J.D. 1994. Gametogenesis in deep- and surface-dwelling oceanic stalked barnacles from the NE Atlantic Ocean. Journal of Experimental Marine Biology and Ecology 184, 142-158.

Gregory, M.R. 1978. Accumulation and distribution of virgin plastic granules on New Zealand beaches. New Zealand Journal of Marine and Freshwater Research 12, 399-414.

Greve, L. 1974. Anatanais normanni (Richardson) found near Bermuda and notes on other Anatanais species. Sarsia 55, 115-120. 


\section{RAFTING OF BENTHIC MARINE ORGANISMS}

Griffiths, C.L. \& Stenton-Dozey, J. 1981. The fauna and rate of degradation of stranded kelp. Estuarine Coastal and Shelf Science 12, 645-653.

Grosholz, E.D. \& Ruiz, G.M. 2003. Biological invasions drive size increases in marine and estuarine invertebrates. Ecology Letters 6, 700-705.

Guilding, L. 1828. Observations on the zoology of the Caribbean Islands. Zoological Journal 3, 403-408.

Guppy, H.B. 1906. Observations of a Naturalist in the Pacific. Vol. II - Plant-Dispersal. London: MacMillan.

Guppy, H.B. 1917. Plants, Seeds and Currents in the East Indies. London: William Norgate.

Gutow, L. 2003a. Local population persistence as a pre-condition for large scale dispersal of Idotea metallica (Crustacea: Isopoda) on drifting habitat patches. Hydrobiologia 503, 45-48.

Gutow, L. 2003b. Konkurrenz, Habitatsegregation und Metapopulationseffekte: Perspektiven für Idotea metallica (Crustacea, Isopoda) in der Nordsee. Ph.D. Thesis, Free University Berlin.

Gutow, L. \& Franke, H.D. 2001. On the current and possible future status of the neustonic isopod Idotea metallica Bosc in the North Sea: a laboratory study. Journal of Sea Research 45, 37-44.

Gutow, L. \& Franke, H.D. 2003. Metapopulation structure of the marine isopod Idotea metallica, a species associated with drifting habitat patches. Helgoland Marine Research 56, 259-264.

Hacker, S.D. \& Madin, L.P. 1991. Why habitat architecture and color are important to shrimps living in pelagic Sargassum: use of camouflage and plant-part mimicry. Marine Ecology Progress Series 70, 143-155.

Hafner, D.J., Riddle, B.R. \& Alvarez-Castaneda, S.T. 2001. Evolutionary relationships of white-footed mice (Peromyscus) on islands in the Sea of Cortez, Mexico. Journal of Mammalogy 82, 775-790.

Hallegraeff, G.M. 1992. On the global spreading of toxic dinoflagellates. Ciguatera Information Bulletin 2, 2-4.

Hamada, T. 1964. Notes on drifted Nautilus in Thailand. Scientific Papers of the College of General Education, University of Tokyo 14, 255-277.

Hardwick-Witman, M.N. 1985. Biological consequences of ice rafting in a New England salt marsh community. Journal of Experimental Marine Biology and Ecology 87, 283-298.

Harrold, C. \& Lisin, S. 1989. Radio-tracking rafts of giant kelp: local production and regional transport. Journal of Experimental Marine Biology and Ecology 130, 237-251.

Hart, M.W., Byrne, M. \& Smith, M.J. 1997. Molecular phylogenetic analysis of life-history evolution in asterinid starfish. Evolution 51, 1848-1861.

Hartnoll, R.G. 2000. Evolution of brachyuran mating behavior: relation to the female molting pattern. In The Biodiversity Crisis and Crustacea. Proceedings of the Fourth International Crustacean Congress. Amsterdam, Netherlands, 20-24 July 1998, Volume 2, J.C. von Vaupel Klein \& F. R. Schram (eds). Rotterdam: A.A. Balkema, 519-525.

Harwell, M.C. \& Orth, R.J. 2002. Long-distance dispersal potential in a marine macrophyte. Ecology 83, 3319-3330.

Häussermann, V. \& Försterra, G. 2001. A new species of sea anemone from Chile, Anemonia alicemartinae n. sp. (Cnidaria: Anthozoa). An invader or an indicator for environmental change in shallow water? Organisms Diversity \& Evolution 1, 211-224.

Hayami, I. \& Kase, T. 1996. Characteristics of submarine cave bivalves in the northwestern Pacific. American Malacological Bulletin 12, 59-65.

Heatwole, H. \& Levins, R. 1972. Biogeography of the Puerto Rican Bank: flotsam transport of terrestrial animals. Ecology 53, 112-117.

Heatwole, H., Sade, D.S. \& Hildreth, R. 1963. Herpetogeography of Puerto Rico. I. Herpetofauna of Cayo Santiago and Cayo Batata. Caribbean Journal of Science 3, 1-5.

Hedgpeth, J.E. 1953. An introduction to the zoogeography of the northwestern Gulf of Mexico with reference to the invertebrate fauna. Publications of the Institute of Marine Science University Texas 3, 111-211.

Helmuth, B., Veit, R.R. \& Holberton, R. 1994. Long-distance dispersal of a sub-Antarctic brooding bivalve (Gaimardia trapesina) by kelp-rafting. Marine Biology 120, 421-426.

Hemsley, W.B. 1885. Introduction. Present state of knowledge of various insular floras, being an introduction to the botany of the Challenger expedition. Report of the Scientific Results of the Voyage of the H.M.S. Challenger. III. Botany, Volume 1.

Hendler, G. 1991. Echinodermata: Ophiuroidea. In Reproduction of Marine Invertebrates, A.C. Giese et al. (eds). Pacific Grove, California: The Boxwood Press, 355-511. 


\section{MARTIN THIEL \& LARS GUTOW}

Hentschel, E. 1922. Über den Bewuchs auf treibenden Tangen der Sargassosee. Mitteilungen des Zoologischen Staatsinstituts und Zoologischen Museums Hamburg 38, 1-26.

Herring, P.J. 1969. Pigmentation and carotenoid metabolism of the marine isopod Idotea metallica. Journal of the Marine Biological Association of the United Kingdom 49, 766-779.

Hicks, G.R.F. 1977. Species composition and zoogeography of marine phytal harpacticoid copepods from Cook Strait, and their contribution to total phytal meiofauna. New Zealand Journal of Marine and Freshwater Research 11, 441-469.

Hicks, G.R.F. 1988. Sediment rafting: a novel mechanism for the small-scale dispersal of intertidal estuarine meiofauna. Marine Ecology Progress Series 48, 69-80.

Highsmith, R.C. 1985. Floating and algal rafting as potential dispersal mechanisms in brooding invertebrates. Marine Ecology Progress Series 25, 169-179.

Hirosaki, Y. 1960. Observations and experiments on the behavior of fishes toward floating objects in aquarium (preliminary report). Journal of the Faculty of Science of the Hokkaido University (Ser. VI) 14, 320-326.

Hirosaki, Y. 1964. Ecological study on fishes with the drifting seaweeds. III. Accompanying animals excluding fishes. Miscellaneous Reports of the Research Institute for Natural Resources, Tokyo 62, 63-70.

Hitchcock, H.B. 1941. The coloration and color changes of the gulf-weed crab, Planes minutus. Biological Bulletin 80, 26-30.

Hobday, A.J. 1998. Faunal patterns and dispersal on kelp rafts in Southern California. Ph.D. Thesis, University of California.

Hobday, A.J. 2000a. Persistence and transport of fauna on drifting kelp (Macrocystis pyrifera (L.) C. Agardh) rafts in the Southern California Bight. Journal of Experimental Marine Biology and Ecology 253, 75-96.

Hobday, A.J. 2000b. Age of drifting Macrocystis pyrifera (L.) C. Agardh rafts in the Southern California Bight. Journal of Experimental Marine Biology and Ecology 253, 97-114.

Hodgson, T.V. 1896. Nautilograpsus minutus, Milne-Edwards: faunistic notes. Journal of the Marine Biological Association of the United Kingdom 4, 178.

Hoek, C.v.d. 1987. The possible significance of long-range dispersal for the biogeography of seaweeds. Helgoländer Meeresuntersuchungen 41, 261-272.

Hoffmann, A.J. 1987. The arrival of seaweed propagules at the shore: a review. Botanica Marina 30, 151-165.

Holdway, P. \& Maddock, L. 1983a. A comparative survey of neuston. Geographical and temporal distribution patterns. Marine Biology 76, 263-270.

Holdway, P. \& Maddock, L. 1983b. Neustonic distributions. Marine Biology 77, 207-214.

Holmquist, J.G. 1994. Benthic macroalgae as a dispersal mechanism for fauna - influence of a marine tumbleweed. Journal of Experimental Marine Biology and Ecology 180, 235-251.

Holmström, A. 1975. Plastic films on the bottom of the Skagerrak. Nature 255, 622-623.

Holmström, C. \& Kjelleberg, S. 1999. Factors influencing the settlement of macrofoulers. In Recent Advances in Marine Biotechnology, Volume 3: Biofilms, Bioadhesion, Corrosion, and Biofouling, M. Fingermann et al. (eds). New Dehli: Science Publishers, Inc., 173-201.

Horn, M.H., Teal, J.M. \& Backus, R.H. 1970. Petroleum lumps on the surface of the sea. Science 168, 245-246.

Horvath, T.G. \& Lamberti, G.A. 1997. Drifting macrophytes as a mechanism for zebra mussel (Dreissena polymorpha) invasion of lake-outlet streams. American Midland Naturalist 138, 29-36.

Hoskin, M.G. 1997. Effects of contrasting modes of larval development on the genetic structures of populations of three species of prosobranch gastropods. Marine Biology 127, 647-656.

Houtman, R., Paul, L.R., Ungemach, R.V. \& Ydenberg, R.C. 1997. Feeding and predator-avoidance by the rose anemone Urticina piscivora. Marine Biology 128, 225-229.

Huber, M.E. 1985. Population genetics of eight species of Trapezia (Brachyura: Xanthidae), symbionts of corals. Marine Biology 85, 23-36.

Hultén, E. 1962. Plants of the floating ice-island Arlis II. Svensk Botanisk Tidskrift 56, 362-364.

Hunter, J.R. \& Mitchell, C.T. 1967. Association of fishes with flotsam in the offshore waters of Central America. Fisheries Bulletin 66, 13-29.

Hurd, C.L., Durante, K.M., Chia, F.S. \& Harrison, P.J. 1994. Effect of bryozoan colonization on inorganic nitrogen acquisition by the kelps Agarum fimbriatum and Macrocystis integrifolia. Marine Biology 121, 167-173. 


\section{RAFTING OF BENTHIC MARINE ORGANISMS}

Hyde, K. 1989. Ecology of tropical marine fungi. Hydrobiologia 178, 199-208.

Hyman, L. 1939. Acoel and polyclad turbellaria from Bermuda and the Sargassum. Bulletin of the Bingham Oceanographic Collection 3, 1-26.

Ida, H., Hiyama, Y. \& Kusaka, T. 1967. Study on fishes gathering around floating seaweed - II. Behavior and feeding habit. Bulletin of the Japanese Society of Science and Fisheries 33, 930-936.

Il'in, I.N. 1992. Pelagic epiboly in the tropical and subtropical waters of the ocean. In Obrastaniye $i$ biopovrezhdenya, I. N. Il'in (ed.). Moscow: Nauka, 77-111.

Il'in, I.N., Makarov, R.R. \& Mileikovsky, S.A. 1977. Fouling of experimental surfaces by goose barnacles and distribution of their larvae in plankton far from shores. Soviet Journal of Marine Biology 3, 154-156.

Il'in, I.N. \& Roginskaya, I.S. 1987. Mollusks-Nudibranchia in pelagic epifauna of solid substrata. In Feeding of Marine Invertebrates and Its Role in Community Formation. Moscow: Inst. Okeanol. Akad. Nauk SSSR, 72-75.

Inglis, G. 1989. The colonisation and degradation of stranded Macrocystis pyrifera (L.) C. Ag. by the macrofauna of a New Zealand sandy beach. Journal of Experimental Marine Biology and Ecology 125, 203-218.

Ingólfsson, A. 1977. Distribution and habitat preferences of some intertidal amphipods in Iceland. Acta Naturalia Islandica 25, 1-28.

Ingólfsson, A. 1992. The origin of the rocky shore fauna of Iceland and the Canadian Maritimes. Journal of Biogeography 19, 705-712.

Ingólfsson, A. 1995. Floating clumps of seaweed around Iceland: natural microcosms and a means of dispersal for shore fauna. Marine Biology 122, 13-21.

Ingólfsson, A. 1998. Dynamics of macrofaunal communities of floating seaweed clumps off western Iceland: a study of patches on the surface of the sea. Journal of Experimental Marine Biology and Ecology 231, 119-137.

Ingólfsson, A. 2000. Colonization of floating seaweed by pelagic and subtidal benthic animals in southwestern Iceland. Hydrobiologia 440, 181-189.

Ingólfsson, A. \& Agnarsson, I. 2003. Amphipods and isopods in the rocky intertidal: dispersal and movements during high tide. Marine Biology 143, 859-866.

Ingólfsson, A. \& Kristjánsson, B.K. 2002. Diet of juvenile lumpsucker Cyclopterus lumpus (Cyclopteridae) in floating seaweed: effects of ontogeny and prey availability. Copeia 2002, 472-476.

Ingólfsson, A. \& Ólafsson, E. 1997. Vital role of drift algae in the life history of the pelagic harpacticoid Parathalestris croni in the northern North Atlantic. Journal of Plankton Research 19, 15-27.

Ingvarson, F. 1903. Origin of driftwood on Jan Mayen Island. Polar Research 17, 125-146.

Jablonski, D. \& Lutz, R.A. 1983. Larval ecology of marine benthic invertebrates; paleobiological implications. Biological Reviews 58, 21-89.

Jackson, J.B.C. 1986. Modes of dispersal of clonal benthic invertebrates: consequences for species' distributions and genetic structure of local populations. Bulletin of Marine Science 39, 588-606.

Jara, C. \& Jaramillo, E. 1979. Report on a finding of Planes marinus Rathbun, 1914, on a [loose] buoy in Maiquillahue, Valdivia province, Chile. Medio Ambiente 4, 108-113.

Johannesson, K. 1988. The paradox of Rockall: why is a brooding gastropod (Littorina saxatilis) more widespread than one having a planktonic larval dispersal stage (L. littorea)? Marine Biology 99, 507-513.

Johannesson, K. 2002. Evolution in Littorina: ecology matters. Journal of Sea Research 49, 107-117.

Johannesson, K. \& Warmoes, T. 1990. Rapid colonization of Belgian breakwaters by the direct developer, Littorina saxatilis (Olivi) (Prosobranchia, Mollusca). Hydrobiologia 193, 99-108.

Johansen, S. \& Hytteborn, H. 2001. A contribution to the discussion of biota dispersal with drift ice and driftwood in the North Atlantic. Journal of Biogeography 28, 105-115.

John, D.M. 1974. New records of Ascophyllum nodosum (L.) de Jol. from the warmer parts of the Atlantic Ocean. Journal of Phycology 10, 243-244.

Johnson, D.L. \& Braman, R.S. 1975. The speciation of arsenic and the content of germanium and mercury in members of the pelagic Sargassum community. Deep-Sea Research 22, 503-507.

Johnson, D.L. \& Richardson, P.L. 1977. On the wind-induced sinking of Sargassum. Journal of Experimental Marine Biology and Ecology 28, 255-267. 


\section{MARTIN THIEL \& LARS GUTOW}

Johnson, M.P., Allcock, A.L., Pye, S.E., Chambers, S.J. \& Fitton, D.M. 2001. The effects of dispersal mode on the spatial distribution patterns of intertidal molluscs. Journal of Animal Ecology 70, 641-649.

Johnson, M.W. \& Olson, J.B. 1948. The life history of a marine harpacticoid copepod, Tisbe furcata (Baird). Biological Bulletin 95, 320-332.

Jokiel, P.L. 1984. Long distance dispersal of reef corals by rafting. Coral Reefs 3, 113-116.

Jokiel, P.L. 1989. Rafting of reef corals and other organisms at Kwajalein Atoll. Marine Biology 101, $483-493$.

Jokiel, P.L. 1990a. Transport of reef corals into the Great Barrier Reef. Nature 347, 665-667.

Jokiel, P.L. 1990b. Long-distance dispersal by rafting: reemergence of an old hypothesis. Endeavour 14, 66-73.

Kaldy, J.E. \& Dunton, K.H. 1999. Ontogenetic photosynthetic changes, dispersal and survival of Thalassia testudinum (turtle grass) seedlings in a sub-tropical lagoon. Journal of Experimental Marine Biology and Ecology 240, 193-212.

Kampf, W.-D., Becker, G. \& Kohlmeyer, J. 1959. Versuche über das Auffinden und den Befall von Holz durch Larven der Bohrmuschel Teredo pedicellata Qutrf. Zeitschrift für Angewandte Zoologie 46, 257-283.

Kaplan, D.M., Largier, J.L., Navarrete, S., Guiñez, R. \& Castilla, J.C. 2003. Large diurnal temperature fluctuations in the nearshore water column. Estuarine and Coastal Shelf Science 57, 385-398.

Kathiresan, K. \& Bingham, B.L. 2001. Biology of mangroves and mangrove ecosystems. Advances in Marine Biology 40, 81-251.

Keller, N.B. 1987. Some data on variation in the biomass of macroepifauna of Sargasso seaweed in the Sargasso Sea. Oceanology 27, 762.

Keough, M.J. \& Chernoff, H. 1987. Dispersal and population variation in the bryozoan Bugula neritina. Ecology 68, 199-210.

Kimura, K., Hotta, H., Fukushima, S.I., Odate, S., Fukuhara, A. \& Naito, M. 1958. Study of the Pacific Saury spawning on the drifting sea-weeds in the Sea of Japan. Bulletin of the Tohoku National Fisheries Research Institute, Shiogama 12, 28-45.

King, W. 1962. The occurrence of rafts for dispersal of land animals into the West Indies. Quarterly Journal of the Florida Academy of Sciences 25, 45-52.

Kingsford, M.J. 1992. Drift algae and small fish in coastal waters of northeastern New Zealand. Marine Ecology Progress Series 80, 41-55.

Kingsford, M.J. 1993. Biotic and abiotic structure in the pelagic environment: importance to small fishes. Bulletin of Marine Science 53, 393-415.

Kingsford, M.J. 1995. Drift algae: a contribution to near-shore habitat complexity in the pelagic environment and an attractant for fish. Marine Ecology Progress Series 116, 297-301.

Kingsford, M.J. \& Choat, J.H. 1985. The fauna associated with drift algae captured with a plankton-mesh purse seine net. Limnology and Oceanography 30, 618-630.

Kingsford, M.J. \& Choat, J.H. 1986. Influence of surface slicks on the distribution and onshore movements of small fish. Marine Biology 91, 161-171.

Kinlan, B.P. \& Gaines, S.D. 2003. Propagule dispersal in marine and terrestrial environments: a community perspective. Ecology 94, 2007-2020.

Kirkman, H. \& Kendrick, G.A. 1997. Ecological significance and commercial harvesting of drifting and beachcast macro-algae and seagrasses in Australia: a review. Journal of Applied Phycology 9, 311-326.

Klima, E. \& Wickham, D. 1971. Attraction of coastal pelagic fishes with artificial structures. Transactions of the American Fisheries Society 100, 86-99.

Knight-Jones, E.W., Knight-Jones, P. \& Vine, P.J. 1972. Anchorage of embryos in Spirorbinae (Polychaeta). Marine Biology 12, 289-294.

Knight-Jones, P. 1978. New Spirorbidae (Polychaeta: Sedentaria) from the East Pacific, Atlantic, Indian and Southern Oceans. Zoological Journal of the Linnean Society 64, 201-240.

Knight-Jones, P. \& Knight-Jones, E.W. 1984. Systematics, ecology and distribution of Southern Hemisphere spirorbids. In Proceedings of the First International Polychaete Conference, P.A. Hutchings (ed.). Sydney, N.S.W.: Linnean Society, 196-210.

Knight-Jones, P., Knight-Jones, E.W., Thorp, C.H. \& Gray, P.W.G. 1975. Immigrant spirorbids (Polychaeta Sedentaria) on the Japanese Sargassum at Portsmouth, England. Zoologica Scripta 4, 145-149.

Knox, G.A. 1954. The intertidal flora and fauna of the Chatham Islands. Nature 174, 871-873. 


\section{RAFTING OF BENTHIC MARINE ORGANISMS}

Kohlmeyer, J. 1972. Parasitic Haloguignardia oceanica (Ascomycetes) and hyperparasitic Sphaceloma cecidii sp. nov. (Deuteromycetes) in drift Sargassum in North Carolina. Journal of the Elisha Mitchell Scientific Society. Chapel Hill NC 88, 255-259.

Kohlmeyer, J. 1984. Tropical marine fungi. Pubblicazioni della Stazione Zoologica di Napoli 5, 329-378.

Kohlmeyer, J., Bebout, B. \& Volkmann-Kohlmeyer, B. 1995. Decomposition of mangrove wood by marine fungi and teredinids in Belize. Marine Ecology 16, 27-39.

Kohlmeyer, J. \& Kohlmeyer, E. 1979. Marine Mycology: The Higher Fungi. New York: Academic Press.

Kornicker, L.S. \& Squires, D.F. 1962. Floating corals: a possible source of erroneous distribution data. Limnology and Oceanography 7, 447-452.

Koutstaal, B.P., Markusse, M.M. \& de Munck, W. 1987. Aspects of seed dispersal by tidal movements. In Vegetation between Land and Sea, A.H.L. Huiskes et al. (eds). Dordrecht: Dr. W. Junk Publishers, 226-233.

Kyle, C.J. \& Boulding, E.G. 2000. Comparative population genetic structure of marine gastropods (Littorina spp.) with and without pelagic larval dispersal. Marine Biology 137, 835-845.

Lam, C., Harder, T. \& Qian, P.Y. 2003. Induction of larval settlement in the polychaete Hydroides elegans by surface-associated settlement cues of marine benthic diatoms. Marine Ecology Progress Series 263, $83-92$.

Lawrence, R.F. 1971. Araneida. In Marion and Prince Edward Islands: Report on the South African Biological and Geological Expedition, 1965-1966, E.M. van Zinderen et al. (eds). Cape Town: A.A. Balkema, 301-313.

Leal, J.H. \& Bouchet, P. 1991. Distribution patterns and dispersal of prosobranch gastropods along a seamount chain in the Atlantic Ocean. Journal of the Marine Biological Association of the United Kingdom 71, $11-25$.

Lee, V.F. 1979. The maritime pseudoscorpions of Baja California, Mexico (Arachnida: Pseudoscorpionida). Occasional Papers of the Californian Academy of Science 131, 1-38.

Levin, L.A. 1984. Life history and dispersal patterns in a dense infaunal polychaete assemblage: community structure and response to disturbance. Ecology 65, 1185-1200.

Levins, R. \& Heatwole, H. 1963. On the distribution of organisms on islands. Caribbean Journal of Science 3, 173-177.

Locke, A. \& Corey, S. 1986. Terrestrial and freshwater invertebrates in the neuston of the Bay of Fundy, Canada. Canadian Journal of Zoology 64, 1535-1541.

Locke, A. \& Corey, S. 1989. Amphipods, isopods and surface currents: a case for passive dispersal in the Bay of Fundy, Canada. Journal of Plankton Research 11, 419-430.

Manooch, C.S., III \& Hogarth, W.T. 1983. Stomach contents and giant trematodes from wahoo, Acanthocybium solanderi, collected along the South Atlantic and Gulf coasts of the United States. Bulletin of Marine Science 33, 227-238.

Marche-Marchad, I. 1968. Remarques sur le développement chez les Cymba, prosobranches volutides et l'hypotehése de leur origine Sud Americaine. Bulletin de Institute Fondamental d'Afrique Noire $\mathbf{3 0}$ A, 1028-1037.

Marcus, E. 1926. Zur Frage der Tierverbreitung und pazifischen Landverbindung. Ergebnisse und Fortschritte der Zoologie 6, 1-26.

Markkaveeva, E.G. 1965. The biocenosis of Sargasso algae in the Red Sea. In Benthos. Kiev: Dumka Nauk, 81-93.

Marko, P.B. 1998. Historical allopatry and the biogeography of speciation in the prosobranch snail genus Nucella. Evolution 52, 757-774.

Martel, A. \& Chia, F.-S. 1991. Drifting and dispersal of small bivalves and gastropods with direct development. Journal of Experimental Marine Biology and Ecology 150, 131-147.

Masó, M., Garces, E., Pages, F. \& Camp, J. 2003. Drifting plastic debris as a potential vector for dispersing Harmful Algal Bloom (HAB) species. Scientia Marina 67, 107-111.

Mason, R. 1961. Dispersal of tropical seeds by ocean currents. Nature 191, 408-409.

Mathieson, A.C., Penniman, C.A., Busse, P.K. \& Tveter-Gallagher, E. 1982. Effects of Ice on Ascophyllum nodosum within the Great Bay estuary system of New Hampshire-Maine. Journal of Phycology 18, 331-336.

Matthew, W.D. 1915. Climate and evolution. Annals of the New York Academy of Sciences 24, 171-318. 


\section{MARTIN THIEL \& LARS GUTOW}

McDonald, G.R. 1983. A review of the nudibranchs of the California coast. Malacologia 24, 114-276.

McInnes, S.J. \& Pugh, P.J.A. 1998. Biogeography of limno-terrestrial tardigrada, with particular reference to the antarctic fauna. Journal of Biogeography 25, 31-36.

Meshaka Jr, W.E., Butterfield, B.P. \& Hauge, J.B. 2004. The Exotic Amphibians and Reptiles of Florida. Malabar, Florida: Krieger Publishing Company.

Michaelsen, W. 1897. Die Polychaetenfauna der deutschen Meere einschliesslich der benachbarten und verbindenden Gebiete. Wissenschaftliche Meeresuntersuchungen 2, 1-217.

Micoletzky, H. 1922. Freilebende Nematoden aus den treibenden Tangen der Sargassosee. Mitteilungen des Hamburger Zoologischen Museums und Institutes 39, 1-11.

Miller, A.W., Hewitt, C.L. \& Ruiz, G.M. 2002. Invasion success: does size really matter? Ecology Letters 5, 159-162.

Miller, K.A., Olsen, J.L. \& Stam, W.T. 2000. Genetic divergence correlated with morphological and ecological subdivision in the deep-water elk kelp, Pelagophycus porra (Phaeophyceae). Journal of Phycology 36, 862-870.

Miller, M.A. 1968. Isopoda and Tanaidacea from buoys in coastal waters of the continental United States, Hawaii, and the Bahamas (Crustacea). Proceedings of the United States National Museum 125, 1-53.

Minchin, D. 1996. Tar pellets and plastics as attachment surfaces for lepadid cirripedes in the North Atlantic Ocean. Marine Pollution Bulletin 32, 855-859.

Mitchell, C.T. \& Hunter, J.R. 1970. Fishes associated with drifting kelp, Macrocystis pyrifera, off the coast of southern California and northern Baja California. California Fish and Game 56, 288-297.

Monge-Nájera, J. 1995. Phylogeny, biogeography and reproductive trends in the Onychophora. Zoological Journal of the Linnean Society (London) 114, 21-60.

Monk, C.R. 1941. Marine harpacticoid copepods from California. Transactions of the American Microscopical Society 60, 75-99.

Moore, C.J., Moore, S.L., Leecaster, M.K. \& Weisberg, S.B. 2001. A comparison of plastic and plankton in the North Pacific central gyre. Marine Pollution Bulletin 42, 1297-1300.

Moore, P.D. 2002. Springboards for springtails. Nature 418, 381.

Morris, B.F., Cadwallader, J., Geiselmann, J. \& Butler, J.N. 1976. Transfer of petroleum and biogenic hydrocarbons in the Sargassum community. In Marine Pollutant Transfer, H.L. Windom \& R.A. Duce (eds). Lexington, Massachusetts: Lexington Books, 235-259.

Morris, B.F. \& Mogelberg, D.D. 1973. Identification manual to the pelagic Sargassum fauna. Bermuda Biological Station Special Publications 11, 1-63.

Morris, R.J. 1974. Lipid composition of surface films and zooplankton from the Eastern Mediterranean. Marine Pollution Bulletin 5, 105-109.

Mortensen, T. 1925. Echinoderms of New Zealand and the Auckland-Campbell Islands. III-V. Asteroidea, Holothuroidea and Crinoidea. Videnskabelige Meddelelser fra Dansk Naturhistorisk Forening $i$ Kjøbenhavn 79, 261-470.

Mortensen, T. 1933. Papers from Dr. Th. Mortensen's Pacific Expedition 1914-16. LXVI. The echinoderms of St. Helena (other than Crinoids). Videnskabelige Meddelelser fra Dansk Naturhistorisk Forening $i$ Kjфbenhavn 93, 401-473.

Mortensen, T. 1941. Echinoderms of Tristan da Cunha. The Results of the Norwegian Scientific Expedition to Tristan da Cunha 1937-1938 7, 1-10.

Morton, B. \& Britton, J.C. 2000. The origins of the coastal and marine flora and fauna of the Azores. Oceanography and Marine Biology: An Annual Review 38, 13-84.

Moser, M.L., Auster, P.J. \& Bichy, J.B. 1998. Effects of mat morphology on large Sargassum-associated fishes: observations from a remotely operated vehicle (ROV) and free-floating video cameras. Environmental Biology of Fishes 51, 391-398.

Muir, J. 1934. The correlation between arrival sites of alien seeds with ocean currents. South African Geographical Journal 17, 23-28.

Muir, J. 1937. The seed-drift of South Africa and some influence of ocean currents on the strand vegetation. Memoirs of the Botanical Survey of South Africa 16, 1-108.

Muñoz, J., Cancino, J.M. \& Molina, M.X. 1991. Effect of encrusting bryozoans on the physiology of their algal substratum. Journal of the Marine Biological Association of the United Kingdom 71, 877-882. 


\section{RAFTING OF BENTHIC MARINE ORGANISMS}

Myers, A.A. 1993. Dispersal and endemicity in gammaridean Amphipoda. Journal of Natural History 27, 901-908.

Nair, N.B. \& Saraswathy, M. 1971. The biology of wood-boring teredinid molluscs. Advances in Marine Biology 9, 335-509.

Nelson, P.A. 2003. Marine fish assemblages associated with fish aggregating devices (FADs): effects of fish removal, FAD size, fouling communities, and prior recruits. Fishery Bulletin 101, 835-850.

Niedbala, W. 1998. Ptyctimous mites of the Pacific Islands. Recent knowledge, origin, descriptions, redescriptions, diagnoses and zoogeography (Acari: Oribatida). Genus 9, 431-458.

Niermann, U. 1986. Distribution of Sargassum natans and some of its epibionts in the Sargasso Sea. Helgoländer Meeresuntersuchungen 40, 343-353.

Nilsson-Cantell, C.A. 1930. Thoracic cirripedes collected in 1925-1927. Discovery Reports 2, 223-260.

Norton, T.A. 1976. Why is Sargassum muticum so invasive? British Phycological Journal 11, 197-198.

Norton, T.A. \& Benson, M.R. 1983. Ecological interactions between the brown seaweed Sargassum muticum and its associated fauna. Marine Biology 75, 169-177.

Norton, T.A. \& Mathieson, A.C. 1983. The biology of unattached seaweeds. In Progress in Phycological Research, F.E. Round \& D.J. Chapman (eds). New York: Elsevier, 333-386.

Ockelmann, K.W. \& Nielsen, C. 1981. On the biology of the prosobranch Lacuna parva in the Oeresund. Ophelia 20, 1-16.

Ó Foighil, D. 1989. Planktotrophic larval development is associated with a restricted geographic range in Lasaea, a genus of brooding, hermaphroditic bivalves. Marine Biology 103, 349-358.

Ó Foighil, D. \& Eernisse, D.J. 1988. Geographically widespread, non-hybridizing, sympatric strains of the hermaphroditic, brooding clam Lasaea in the northeastern Pacific Ocean. Biological Bulletin (Woods Hole) 175, 218-229.

Ó Foighil, D. \& Jozefowicz, C.J. 1999. Amphi-Atlantic phylogeography of direct-developing lineages of Lasaea, a genus of brooding bivalves. Marine Biology 135, 115-122.

Ó Foighil, D., Marshall, B.A., Hilbish, T.J. \& Pino, M.A. 1999. Trans-Pacific range extension by rafting is inferred for the flat oyster Ostrea chilensis. Biological Bulletin (Woods Hole) 196, 122-126.

O’Hara, T. 1998. Origin of Macquarie Island echinoderms. Polar Biology 20, 143-151.

Ojeda, F.P. \& Santelices, B. 1984. Invertebrate communities in holdfasts of the kelp Macrocystis pyrifera from southern Chile. Marine Ecology Progress Series 16, 65-73.

Ólafsson, E., Ingólfsson, A. \& Steinarsdottir, M.B. 2001. Harpacticoid copepod communities of floating seaweed: controlling factors and implications for dispersal. Hydrobiologia 453, 189-200.

Oliveira, E., Ugadim, Y. \& Paula, E. 1979. Associated epibiota on Sargassum floating on the waters of the Brazilian current — biogeographical remarks. Boletim do Botanica, Universidade de Sao Paulo 7, 5-9.

Olsson, M. \& Madsen, T. 1998. Sexual selection and sperm competition in reptiles. In Sperm Competition and Sexual Selection, T.R. Birkhead \& A.P. Moller (eds). San Diego: Academic Press, 503-577.

Orrell, K.S. \& Jenssen, T.A. 2002. Male mate choice by the lizard Anolis carolinensis: a preference for novel females. Animal Behaviour 63, 1091-1102.

Orth, R.J., Luckenbach, M. \& Moore, K.A. 1994. Seed dispersal in a marine macrophyte: implications for colonization and restoration. Ecology 75, 1927-1939.

Ottaway, J.R. \& Kirby, G.C. 1975. Genetic relationships between brooding and brooded Actinia tenebrosa. Nature 255, 221-223.

Page, T.J. 2001. A molecular systematic investigation of the Limatula ovalis/pygmaea species complex (Bivalvia: Limidae) in the Southern Ocean. M.Sc. Thesis, Imperial College, University of London.

Page, T.J. \& Linse, K. 2002. More evidence of speciation and dispersal across the Antarctic Polar Front through molecular systematics of Southern Ocean Limatula (Bivalvia: Limidae). Polar Biology 25, 818-826.

Palmer, A.R. \& Strathmann, R.R. 1981. Scale of dispersal in vaying environments and its implications for life histories of marine invertebrates. Oecologia 48, 308-318.

Palmer, M.A. 1988. Dispersal of marine meiofauna: a review and conceptual model explaining passive transport and active emergence with implications for recruitment. Marine Ecology Progress Series 48, 81-91.

Palumbi, S.R. 2003. Population genetics, demographic connectivity, and the design of marine reserves. Ecological Applications 13, 159-169. 


\section{MARTIN THIEL \& LARS GUTOW}

Parker, T. \& Tunnicliffe, V. 1994. Dispersal strategies of the biota on an oceanic seamount — implications for ecology and biogeography. Biological Bulletin 187, 336-345.

Parr, A.E. 1939. Quantitative observations on the pelagic Sargassum vegetation of the western North Atlantic. Bulletin of the Bingham Oceanographic Collection 6, 1-94.

Patel, B. 1959. The influence of temperature on the reproduction and moulting of Lepas anatifera L. under laboratory conditions. Journal of the Marine Biological Association of the United Kingdom 38, 589-597.

Pavia, H., Carr, H. \& Aberg, P. 1999. Habitat and feeding preferences of crustacean mesoherbivores inhabiting the brown seaweed Ascophyllum nodosum (L.) Le Jol. and its epiphytic macroalgae. Journal of Experimental Marine Biology and Ecology 236, 15-32.

Peck, S.B. 1994. Diversity and zoogeography of the non-oceanic Crustacea of the Galapagos-Islands, Ecuador (Excluding Terrestrial Isopoda). Canadian Journal of Zoology-Revue Canadienne de Zoologie 72, 54-69.

Pethon, P. 1970. Two species of isopoda new to Norway. Rhizocrinus 1, 1-4.

Phillips, R.C. 1963. Ecology of floating algal communities in Florida. Quarterly Journal of the Florida Academy of Science 26, 329-334.

Pole, M. 1994. The New Zealand flora — entirely long-distance dispersal. Journal of Biogeography 21, 625-635.

Polunin, N. 1955. Long-distance plant dispersal in the north polar regions. Nature 176, 22-24.

Prasannarai, K. \& Sridhar, K.R. 1997. Effect of incubation period of driftwood on the occurrence of marine fungi. Indian Journal of Marine Sciences 26, 380-382.

Prescott, J.H. 1959. Rafting of jack rabbit on kelp. Journal of Mammalogy 18, 40, 443-444.

Prins, T.C., Smaal, A.C. \& Dame, R.F. 1998. A review of the feedbacks between bivalve grazing and ecosystem processes. Aquatic Ecology 31, 346-359.

Pugh, P.J.A. 2004. Biogeography of spiders (Araneae: Arachnida) on the islands of the Southern Ocean. Journal of Natural History 38, 1461-1487.

Pugh, P.J.A., Dartnall, H.J.G. \& McInnes, S.J. 2002. The non-marine Crustacea of Antarctica and the islands of the Southern Ocean: biodiversity and biogeography. Journal of Natural History 36, 1047-1103.

Pugh, P.J.A. \& Scott, B. 2002. Biodiversity and biogeography of non-marine Mollusca on the islands of the Southern Ocean. Journal of Natural History 36, 927-952.

Raxworthy, C.J., Forstner, M.R. \& Nussbaum, R.A. 2002. Chameleon radiation by oceanic dispersal. Nature 415, 784-787.

Reed, D.C., Amsler, C.D. \& Ebeling, A.W. 1992. Dispersal in kelps: factors affecting spore swimming and competency. Ecology 73, 1577-1585.

Reed, D.C., Laur, D.R. \& Ebeling, A.W. 1988. Variation in algal dispersal and recruitment: the importance of episodic events. Ecological Monographs 58, 321-335.

Reeder, T.W., Cole, C.J. \& Dessauer, H.C. 2002. Phylogenetic relationships of whiptail lizards of the genus Cnemidophorus (Squamata: Teiidae): a test of monophyly, reevaluation of karyotypic evolution, and review of hybrid origins. American Museum Novitates 3365, 1-61.

Reid, D.G. 1986. The Littorinid Molluscs of Mangrove Forests in the Indo-Pacific Region: The Genus Littoraria. London: British Museum of Natural History.

Reid, D.G. 1999. The genus Littoraria Griffith \& Pidgeon, 1834 (Gastropoda: Littorinidae) in the tropical Eastern Pacific. Veliger 42, 21-53.

Reid, D.G. 2002. The genus Nodilittorina von Martens, 1897 (Gastropoda: Littorinidae) in the eastern Pacific Ocean, with a discussion of biogeographic provinces of the rocky-shore fauna. Veliger 45, 85-170.

Reid, D.G. \& Geller, J.B. 1997. A new ovoviviparous species of Tectarius (Gastropoda: Littorinidae) from Niue, South Pacific, with a molecular phylogeny of the genus. Journal of Molluscan Studies 63, 207-233.

Reineck, H.-E. 1976. Drift ice action on tidal flats, North Sea. Revue de Géographie de Montreal 30, 197-200.

Reusch, T.B.H. 2002. Microsatellites reveal high population connectivity in eelgrass (Zostera marina) in two contrasting coastal areas. Limnology and Oceanography 47, 78-85.

Reusch, T.B.H., Stam, W.T. \& Olsen, J.L. 1999. Size and estimated age of genets in eelgrass, Zostera marina, assessed with microsatellite markers. Marine Biology 133, 519-525.

Reznichenko, O.G. 1981. Multidisciplinary studies of the floating epibolic biochore in the Northwestern oart of the Pacific Ocean. In Epiboly and Biological Corrosion in Aquatic Medium. N.A. Rudyakova (ed.). Moscow: Nauka, 109-126. 


\section{RAFTING OF BENTHIC MARINE ORGANISMS}

Ribera, M.A. \& Boudouresque, C.F. 1995. Introduced marine plants with special reference to macroalgae: mechanisms and impact. Progress in Phycological Research 11, 187-268.

Richards, A.F. 1958. Transpacific distribution of floating pumice from Isla San Benedicto, Mexico. Deep-Sea Research 5, 29-35.

Richardson, H. 1905. A monograph on the Isopods of North America. Bulletin of the United States National Museum 54, 1-727.

Richmond, R.H. 1987. Energetics, competency, and long-distance dispersal of planula larvae of the coral Pocillopora damicornis. Marine Biology 93, 527-533.

Rieppel, O. 2002. A case of dispersing chameleons. Nature 415, 744-745.

Ritchie, J.S. 1908. On collections of the Cape Verde Islands marine fauna, made by Cyril Crossland, July to September, 1904. The hydroids. Proceedings of the Zoological Society of London 1907, 488-514.

Rodríguez, G. \& Suárez, H. 2001. Anthropogenic dispersal of decapod Crustaceans in aquatic environments. Interciencia 26, 282-288.

Rosewater, J. 1975. An annotated list of the marine mollusks of Ascension Island, South Atlantic Ocean. Smithsonian Contribution to Zoology 189, 1-41.

Rouch, J. 1954. Les Îles flottantes. Archiv für Meteorologie Geophysik und Bioklimatologie Series A, 7, 528-532.

Ruckelshaus, M.H. 1996. Estimation of genetic neighborhood parameters from pollen and seed dispersal in the marine angiosperm Zostera marina L. Evolution 50, 856-864.

Ruiz, G.M., Fofonoff, P.W., Carlton, J.T., Wonham, M.J. \& Hines, A.H. 2000. Invasion of coastal marine communities in North America: apparent patterns, processes, and biases. Annual Review of Ecology and Systematics 31, 481-531.

Russo, C.A.M., Sole-Cava, A.M. \& Thorpe, J.P. 1994. Population structure and genetic variation in two tropical sea anemones (Cnidaria, Actinidae) with different reproductive strategies. Marine Biology 119, 267-276.

Ryland, J.S. 1974. Observations on some epibionts of gulfweed, Sargassum natans (L.) Meyen. Journal of Experimental Marine Biology and Ecology 14, 17-25.

Safran, P. \& Omori, M. 1990. Some ecological observations on fishes associated with drifting seaweed off Tohoku coast, Japan. Marine Biology 105, 395-402.

Salazar-Vallejo, S.I. 1992. Dos nuevos registros de Anfinomidos (Polychaeta: Amphinomidae) para el Mar Caribe, con notas descriptivas para ambas especies. Caribbean Journal of Science 28, 216-217.

Sano, M., Omori, M. \& Taniguchi, K. 2003. Predator-prey systems of drifting seaweed communities off the Tohoku coast, northern Japan, as determined by feeding habit analysis of phytal animals. Fisheries Science 69, 260-268.

Scheibling, R.E., Hennigar, A.W. \& Balch, T. 1999. Destructive grazing, epiphytism, and disease: the dynamics of sea urchin-kelp interactions in Nova Scotia. Canadian Journal of Fisheries and Aquatic Science 56, 2300-2314.

Scheltema, R.S. 1977. Dispersal of marine invertebrate organisms: paleobiogeographic and biostratigraphic implications. In Concepts and Methods of Biostratigraphy, E.G. Kauffmann \& J.E. Hazel (eds). Stroudsberg, Pennsylvania: Dowden, Hutchinson \& Ross, 73-108.

Scheltema, R.S. 1988. Initial evidence for the transport of teleplanic larvae of benthic invertebrates across the East Pacific barrier. Biological Bulletin (Woods Hole) 174, 145-152.

Scheltema, R.S. 1995. The relevance of passive dispersal for the biogeography of Caribbean mollusks. American Malacological Bulletin 11, 99-115.

Schiel, D.R. 2004. The structure and replenishment of rocky shore intertidal communities and biogeographic comparisons. Journal of Experimental Marine Biology and Ecology 300, 309-342.

Schneider, F.I. \& Mann, K.H. 1991. Rapid recovery of fauna following simulated ice-rafting in a Nova Scotia seagrass bed. Marine Ecology Progress Series 78, 57-70.

Schrödl, M. 1999a. Hancockia schoeferti, spec. nov., a new dendronotoidean nudibranch species from Central Chile. Spixiana 22, 247-254.

Schrödl, M. 1999b. Zoogeographic relationships of the Magellan Nudibranchia (Mollusca: Opisthobranchia), with special reference to species of adjacent regions. Scientia Marina 63 (Suppl. 1), 409-416.

Schrödl, M. 2003. Sea Slugs of Southern South America. Hackenheim: ConchBooks. 


\section{MARTIN THIEL \& LARS GUTOW}

Schubart, C.D., González-Gordillo, J.I., Reyns, N.B., Liu, H.-C. \& Cuesta, J.A. 2001. Are Atlantic and IndoPacific populations of the rafting crab, Plagusia depressa (Fabricius), distinct? New evidence from larval morphology and mtDNA. The Raffles Bulletin of Zoology 49, 301-310.

Schwamborn, R. \& Bonecker, A.C.T. 1996. Seasonal changes in the transport and distribution of meroplankton into a Brazilian estuary with emphasis on the importance of floating mangrove leaves. Arquivos de Biologia e Tecnologia 39, 451-462.

Segawa, S., Sawada, T., Higaki, M., Yoshida, T. \& Kamura, S. 1961. The floating seaweeds of the sea to the west of Kyushu. Records Oceanographic Works in Japan Special Number 5, 179-186.

Segawa, S., Sawada, T., Higaki, M., Yoshida, T., Ohshiro, H. \& Hayashida, F. 1962. Some comments on the movement of the floating seaweeds. Records of Oceanographic Works in Japan 6, 153-159.

Seidel, D. 2002. Zur Verbreitungsökologie der Flachen Strandschnecke Littorina fabalis im Sylter Wattenmeer. Diploma Thesis, University of Hamburg.

Sever, D.M. \& Hamlett, W.C. 2002. Female sperm storage. Journal of Experimental Zoology 292, 187-199.

Sewell, M.A. 1994. Birth, recruitment and juvenile growth in the intraovarian brooding sea cucumber Leptosynapta clarki. Marine Ecology Progress Series 114, 149-156.

Shaffer, J.A., Doty, D.C., Buckley, R.M. \& West, J.E. 1995. Crustacean community composition and trophic use of the drift vegetation habitat by juvenile splitnose rockfish Sebastes diploproa. Marine Ecology Progress Series 123, 13-21.

Shanks, A.L. 1985. Behavioral basis of internal-wave-induced shoreward transport of megalopae of the crab Pachygrapsus crassipes. Marine Ecology Progress Series 24, 289-295.

Shanks, A.L. 2002. Internal tides and the biology of continental shelf waters. In The Oceanography and Ecology of the Nearshore and Bays in Chile. Proceeding of the International Symposium on Linkages and Dynamics of Coastal System: Open Coasts and Embayments, Santiago, Chile 2000, J.C. Castilla \& J.L. Largier (eds). Santiago, Chile: Ediciones Universidad Católica de Chile, 3-27.

Shanks, A.L., Grantham, B.A. \& Carr, M.H. 2003. Propagule dispersal distance and the size and spacing of marine reserves. Ecological Applications 13, 159-169.

Shaw, D.G. \& Mapes, G.A. 1979. Surface circulation and the distribution of pelagic tar and plastic. Marine Pollution Bulletin 10, 160-162.

Shick, J.M., Hoffmann, R.J. \& Lamb, A.N. 1979. Asexual reproduction, population structure, and genotypeenvironment interactions in sea anemones. American Zoologist 19, 699-713.

Shirley, T.C. 1974. Planes cyaneus Dana, 1852 (Decapoda, Grapsidae) from Padre Island, Texas: a new record for the Gulf of Mexico and the North Atlantic. Crustaceana 26, 107-108.

Shumway, G.A. 1953. Rafted pebbles from the deep ocean off Baja California. Journal of Sedimentary Petrology 23, 24-33.

Si, A., Alexander, C.G. \& Bellwood, O. 2000. Habitat partitioning by two wood-boring invertebrates in a mangrove system in tropical Australia. Journal of the Marine Biological Association of the United Kingdom 80, 1131-1132.

Sieburth, J.M. \& Conover, J.T. 1965. Sargassum tannin, an antibiotic which retards fouling. Nature 208, 52-53.

Sieburth, J.M. \& Jensen, A. 1969. Studies on algal substances in the sea. II. The formation of Gelbstoff (humic material) by exudates of Phaeophyta. Journal of Experimental Marine Biology and Ecology 3, 275-289.

Siegel, D.A., Kinlan, B.P., Gaylord, B. \& Gaines, S.D. 2003. Lagrangian descriptions of marine larval dispersion. Marine Ecology Progress Series 260, 83-96.

Simpson, R.D. 1977. The reproduction of some littoral molluscs from Macquarie Island (sub-Antarctic). Marine Biology 44, 125-142.

Sinitsyn, V.O. \& Reznichenko, O.G. 1981. Fouling of small-sized driftwood in the Western Pacific Ocean. In Ekologiya Massovykh vidov Okeanicheskogo Obrastaniya (Ecology of Common Species of Ocean Fouling) O.G. Reznichenko (ed.). Moscow: Nauka, 18-69.

Skarpaas, O. \& Stabbetorp, E. 2001. Diaspore ecology of Mertensia maritima: effects of physical treatments and their relative timing on dispersal and germination. Oikos 95, 374-382.

Skerman, T.M. 1958. Rates of growth in two species of Lepas (Cirripedia). New Zealand Journal of Science 1, 402-411.

Smith, C.M. \& Walters, L.J. 1999. Fragmentation as a strategy for Caulerpa species: fates of fragments and implications for management of an invasive weed. Marine Ecology 20, 307-319. 


\section{RAFTING OF BENTHIC MARINE ORGANISMS}

Smith, K.L., Burns, K.A. \& Carpenter, E.J. 1973. Respiration of the pelagic Sargassum community. DeepSea Research Oceanographic Abstracts 20, 213-217.

Smith, K.M. 1994. Short-term dynamics of tropical and macroalgal epifauna: patterns and processes in recolonisation of Sargassum fissifolium. Marine Ecology Progress Series 110, 177-185.

Smith, S.D.A. \& Simpson, R.D. 1995. Effects of the Nella Dan oil spill on the fauna of Durvillaea antarctica holdfasts. Marine Ecology Progress Series 121, 73-89.

Smith, W.G. 1968. A neonate Atlantic loggerhead turtle (Caretta caretta caretta) captured at sea. Copeia 1968, $880-881$.

Sokolowsky, A. 1912. Über drei in der Nordsee bei Helgoland gefundene Caprelliden. Wissenschaftliche Meeresuntersuchungen 5, 11-14.

Sorg, M.H., Dearborn, J.H., Monahan, E.I., Ryan, H.F., Sweeney, K.G. \& David, E. 1997. Forensic taphonomy in marine contexts. In Forensic Taphonomy: The Postmortem Fate of Human Remains, W.D. Haglund \& M.H. Sorg (eds). Boca Raton, Florida.: CRC Press, 567-604.

Spindler, M. 1980. The pelagic gulf weed Sargassum natans as a habitat for the benthic foraminifera Planorbulina acervalis and Rosalina globularis. Neues Jahrbuch für Geologie und Paläontologie. Monatshefte 9, 569-580.

Spivak, E.D. \& Bas, C.C. 1999. First finding of the pelagic crab Planes marinus (Decapoda: Grapsidae) in the southwestern Atlantic. Journal of Crustacean Biology 19, 72-76.

Sponer, R. \& Roy, M.S. 2002. Phylogeographic analysis of the brooding brittle star Amphipholis squamata (Echinodermata) along the coast of New Zealand reveals high cryptic genetic variation and cryptic dispersal potential. Evolution 56, 1954-1967.

Steele, D.H. \& Steele, V.J. 1972. The biology of Gammarellus angulosus (Crustacea, Amphipoda) in the Northwestern Atlantic. Journal of Fisheries Research Board of Canada 29, 1337-1340.

Stegenga, H. \& Mol, I. 1983. Flora van de Nederlandse zeewieren. Amsterdam: Koninklijke Nederlandse Natuurhistorische Vereniging.

Steiner, G. 1915. Freilebende Nematoden von der Küste Sumatras. Zoologische Jahrbücher Abteilung Systematik 38, 223-244.

Steinke, T.D. 1986. A preliminary study of buoyancy behaviour in Avicennia marina propagules. South African Journal of Botany 52, 559-565.

Steinke, T.D. \& Ward, C.J. 2003. Use of plastic drift cards as indicators of possible dispersal of propagules of the mangrove Avicennia marina by ocean currents. African Journal of Marine Science 25, 169-176.

Stevens, L.M., Gregory, M.R. \& Foster, B.A. 1996. Fouling bryozoans on pelagic and moored plastics from northern New Zealand. In Bryozoans in Space and Time, D.P. Gordon et al. (eds). Wellington: NIWA, 321-340.

Stieglitz, T. \& Ridd, P.V. 2001. Trapping of mangrove propagules due to density-driven secondary circulation in the Normanby River estuary, NE Australia. Marine Ecology Progress Series 211, 131-142.

St. John, S. 1862. Life in the Forests of the Far East, Vol. 1. London: Smith, Elder \& Company.

Stoddart, J.A. 1983. Asexual production of planulae in the coral Pocillopora damicornis. Marine Biology 76, 279-284.

Stoner, A.W. 1983. Pelagic Sargassum: evidence for a major decrease in biomass. Deep-Sea Research 30, 469-474.

Stoner, A.W. \& Greening, H.S. 1984. Geographic variation in the macrofaunal associates of pelagic Sargassum and some biogeographic implications. Marine Ecology Progress Series 20, 185-192.

Stoner, A.W. \& Humphris, S.E. 1985. Abundance and distribution of Halobates micans (Insecta: Gerridae) in the Northwest Atlantic. Deep-Sea Research 32, 733-739.

Strathmann, R.R. \& Strathmann, M.F. 1982. The relationship between adult size and brooding in marine invertebrates. American Naturalist 119, 91-101.

Sundari, R., Vikineswary, S. \& Yusoff, M. 1995. Marine fungi on driftwood collected from the sandy beaches of Port Dickson, Malaysia. Journal of Bioscience (Penang) 6, 25-29.

Sundari, R., Vikineswary, S., Yusoff, M. \& Jones, E.B.G. 1996. Observations on tropical arenicolous marine fungi on driftwood from Malaysia and Singapore. Botanica Marina 39, 327-333.

Svavarsson, J. 1979. Ný Panglús (Synidotea bicuspida (Owen)) fundin vid Ísland. Náttúrufraedingurinn 49, 190-193. 


\section{MARTIN THIEL \& LARS GUTOW}

Svavarsson, J. 1982. Limnoria borealis (Isopoda, Flabellifera) and its commensal, Caecijaera borealis (Isopoda, Asellota), found in Icelandic waters. Sarsia 67, 223-226.

Svavarsson, J., Osore, M.K.W. \& Ólafsson, E. 2002. Does the wood-borer Sphaeroma terebrans (Crustacea) shape the distribution of the mangrove Rhizophora mucronata. Ambio 31, 574-579.

Swearer, S.E., Shima, J.S., Hellberg, M.E., Thorrold, S.R., Jones, G.P., Robertson, D.R., Morgan, S.G., Selkoe, K.A., Ruiz, G.M. \& Warner, R.R. 2002. Evidence of self-recruitment in demersal marine populations. Bulletin of Marine Science 70, 251-271.

Takeuchi, I. \& Hirano, R. 1996. Clinging behaviour of the epifaunal caprellids (Amphipoda) inhabiting the Sargassum zone on the Pacific coast of Japan, with its evolutionary implications. Journal of Crustacean Biology 15, 481-492.

Takeuchi, I. \& Sawamoto, S. 1998. Distribution of caprellid amphipods (Crustacea) in the western North Pacific based on the CSK International Zooplankton Collection. Plankton Biology and Ecology 45, 225-230.

Tanaka, M.O. \& Leite, F.P.P. 2003. Spatial scaling in the distribution of macrofauna associated with Sargassum stenophylum (Mertens) Martius: analyses of faunal groups, gammarid life habits, and assemblage structure. Journal of Experimental Marine Biology and Ecology 293, 1-22.

Tanigawa, T. 2001. Occurrence of juvenile swimming crab Portunus trituberculatus in drifting seaweeds in Hiuchi-nada, Seto Inland Sea. Bulletin of the Ehime Prefectural Fisheries Experiment Station 9, 25-32.

Taylor, R.B. 1998. Short-term dynamics of a seaweed epifaunal assemblage. Journal of Experimental Marine Biology and Ecology 227, 67-82.

Taylor, R.B. \& Rees, T.A.V. 1998. Excretory products of mobile epifauna as a nitrogen source for seaweeds. Limnology and Oceanography 43, 600-606.

Thiel, M. 2003a. Extended parental care in crustaceans — an update. Revista Chilena de Historia Natural 76, 205-218.

Thiel, M. 2003b. Reproductive biology of Limnoria chilensis: another boring peracarid species with extended parental care. Journal of Natural History 37, 1713-1726.

Thiel, M. 2003c. Rafting of benthic macrofauna: important factors determining the temporal succession of the assemblage on detached macroalgae. Hydrobiologia 503, 49-57.

Thiel, M., Guerra-García, J.M., Lancellotti, D. \& Vásquez, N. 2003. The distribution of littoral caprellids (Crustacea: Amphipoda: Caprellidea) along the Pacific coast of continental Chile. Revista Chilena De Historia Natural 76, 297-312.

Thiel, M. \& Gutow, L. 2004. The ecology of rafting in the marine environment. I. The floating substrata. Oceanography and Marine Biology: An Annual Review 42, 181-263.

Thiel, M. \& Vásquez, J.A. 2000. Are kelp holdfasts islands on the ocean floor? Indication for temporarily closed aggregations of peracarid crustaceans. Hydrobiologia 440, 45-54.

Thörner, E. \& Ankel, W.E. 1966. Die Entenmuschel Lepas fascicularis in der Nordsee. Natur und Museum 96, 209-220.

Thornton, I.W.B. 1997. Krakatau: The Destruction and Reassembly of an Island Ecosystem. Cambridge, Massachusetts: Harvard University Press.

Thulin, G. 1942. Ein neuer mariner Tardigrad. Göteborgs Kungliga Vetenskaps- och Vitterhets-Samhälles handlingar 2, 1-10.

Timmermann, G. 1932. Biogeographische Untersuchungen über die Lebensgemeinschaft des treibenden Golfkrautes. Zeitschrift der Morphologie und Ökolologie der Tiere 25, 288-335.

Todd, C.D., Lambert, W.J. \& Thorpe, J.P. 1998. The genetic structure of intertidal populations of two species of nudibranch molluscs with planktotrophic and pelagic lecithotrophic larval stages: are pelagic larvae "for" dispersal? Journal of Experimental Marine Biology and Ecology 228, 1-28.

Tsikhon-Lukanina, E.A., Nikolaeva, G.G. \& Reznichenko, O.G. 1986. Feeding spectra of oceanic epiboles. Okeanologiya 26, 1006-1009.

Tsikhon-Lukanina, E.A., Reznichenko, O.G. \& Lukasheva, T.A. 2001b. Feeding and spawning of the barnacle Lepas anatifera (Cirripedia, Lepadidae) on floating substrates in the open northwestern Pacific Ocean. Zoologicheskii Zhurnal 80, 650-655.

Tsikhon-Lukanina, E.A., Reznichenko, O.G. \& Nikolaeva, G.G. 2001a. Ecology of invertebrates on the oceanic floating substrata in the Northwest Pacific Ocean. Russian Academy of Sciences. Oceanology 41, 525-530. 


\section{RAFTING OF BENTHIC MARINE ORGANISMS}

Tsikhon-Lukanina, E.A., Soldatova, I.N., Kuznetsova, I.A. \& Il'in, I.N. 1976. Makroepiboly in the Strait of Tunis. Okeanologiya 16, 907-911.

Tully, O. \& Ó Céidigh, P. 1986a. The ecology of Idotea species (Isopoda) and Gammarus locusta (Amphipoda) on surface driftweed in Galway Bay (West of Ireland). Journal of the Marine Biological Association of the United Kingdom 66, 931-942.

Tully, O. \& Ó Ceidigh, P. 1986b. Density variations and population structure of Eurydice inermis and E. truncata (Isopoda: Cirolanidae) in the neuston of Galway Bay (Ireland). Cahiers de Biologie Marine 27, 225-233.

Turner R.D. 1966. A Survey and Illustrated Catalogue of the Teredinidae (Mollusca; Bivalvia). Cambridge: The Museum of Comparative Zoology.

Uchida, K. \& Shojima, Y. 1958. Studies on the larvae and juveniles of fish accompanying floating algae. I. Research in the vicinity of Tsuyazaki, during Mar. 1957-Mar. 1958. Bulletin of the Japanese Society of Science and Fisheries 24, 411-415.

Vader, W. 1983. Prehensile pereopods in gammaridean Amphipoda. Sarsia 68, 139-148.

Vallentin, R. 1895. Some remarks on the dispersal of marine animals by means of seaweeds. The Annals and Magazine of Natural History, Zoology, Botany and Geology 16, 418-423.

Van der Baan, S.M. \& Holthuis, L.B. 1969. On the occurrence of Isopoda in the surface plankton in the North Sea near the lightship "Texel”. Netherlands Journal of Sea Research 4, 354-363.

Van der Baan, S.M., Holthuis, L.B. \& Schrieken, B. 1972. Decapoda and decapod larvae in the surface plankton from the southern North Sea near 'Texel' lightship. Zoologische Bijdragen 13, 75-97.

Van Duzer, C. 2004. Floating Islands: A Global Bibliography. Los Altos Hills, California: Cantor Press.

Vásquez, J.A. 1993. Effects on the animal community of dislodgment of holdfasts of Macrocystis pyrifera. Pacific Science 47, 180-184.

Vermeij, G.J., Palmer, A.R. \& Lindberg, D.R. 1990. Range limits and dispersal of mollusks in the Aleutian Islands, Alaska. Veliger 33, 346-354.

Virnstein, R.W. \& Curran, M.C. 1986. Colonization of artificial seagrass versus time and distance from source. Marine Ecology Progress Series 29, 279-288.

Volkmann-Kohlmeyer, B. \& Kohlmeyer, J. 1993. Biogeographic observations on Pacific marine fungi. Mycologia 85, 337-346.

Wahle, R.A. \& Gilbert, A.E. 2002. Detecting and quantifying male sea urchin spawning with time-integrated fertilization assays. Marine Biology 140, 375-382.

Wakabara, Y., Tararam, A.S. \& Takeda, A.M. 1983. Comparative study of the amphipod fauna living on Sargassum of two Itanhaem shores, Brazil. Journal of Crustacean Biology 3, 602-607.

Walters, K. \& Bell, S.S. 1986. Diel patterns of active vertical migration in seagrass meiofauna. Marine Ecology Progress Series 34, 95-103.

Wares, J.P. 2001. Intraspecific variation and geographic isolation in Idotea balthica (Isopoda: Valvifera). Journal of Crustacean Biology 21, 1007-1013.

Wares, J.P. \& Cunningham, C.W. 2001. Phylogeography and historical ecology of the North Atlantic intertidal. Evolution 55, 2455-2469.

Waters, J.M. \& Roy, M.S. 2003. Marine biogeography of southern Australia: phylogeographical structure in a temperate sea star. Journal of Biogeography 30, 1787-1796.

Waters, J.M. \& Roy, M.S. 2004. Out of Africa: the slow train to Australasia. Systematic Biology 53, 18-24.

Watts, P.C., Thorpe, J.P. \& Taylor, P.D. 1998. Natural and anthropogenic dispersal mechanisms in the marine environment: a study using cheilostome Bryozoa. Philosophical Transactions of the Royal Society of London Series B 353, 453-464.

Wehrtmann, I.S. \& Dittel, A.I. 1990. Utilization of floating mangrove leaves as a transport mechanism of estuarine organisms, with emphasis on decapod Crustacea. Marine Ecology Progress Series 60, 67-73.

Weis, J.S. 1968. Fauna associated with pelagic Sargassum in the Gulf Stream. American Midland Naturalist 80, 554-558.

Wheeler, W.M. 1916. Ants carried in a floating log from the Brazilian coast to San Sebastian Island. Psyche 23, 180-183. 


\section{MARTIN THIEL \& LARS GUTOW}

Wickham, D.E. 1979. The relationship between megalopae of the Dungeness crab, Cancer magister, and hydroid, Velella velella, and its influence on abundance estimates of C. magister megalopae. California Fish and Game 65, 184-186.

Wikström, S.A. \& Pavia, H. 2004. Chemical settlement inhibition versus post-settlement mortality as an explanation for differential fouling of two congeneric seaweeds. Oecologia 138, 223-230.

Wild, C., Huettel, M., Klueter, A., Kremb, S.G., Rasheed, M.Y.M. \& Jorgensen, B.B. 2004. Coral mucus functions as an energy carrier and particle trap in the reef ecosystem. Nature 428, 66-70.

Wilhelmsen, U. 1999. Rapid colonization of new habitats in the Wadden Sea by the ovoviviparous Littorina saxatilis (Olivi). Helgoländer Meeresuntersuchungen 52, 325-335.

Winkworth, R.C., Wagstaff, S., Glenny, D. \& Lockhart, P.J. 2002. Plant dispersal N.E.W.S. from New Zealand. Trends in Ecology and Evolution 17, 514-520.

Winston, J.E. 1982. Drift plastic — an expanding niche for a marine invertebrate. Marine Pollution Bulletin 13, 348-351.

Winston, J.E., Gregory, M.R. \& Stevens, L.M. 1997. Encrusters, epibionts, and other biota associated with pelagic plastics: a review of biogeographical, environmental, and conservation issues. In Marine Debris: Sources, Impacts, and Solution, J.M. Coe \& D.B. Rogers (eds). New York: Springer, 81-97.

Witherington, B.E. 2002. Ecology of neonate loggerhead turtles inhabiting lines of downwelling near a Gulf Stream front. Marine Biology 140, 843-853.

Wittmann, O. 1934. Die biogeographischen Beziehungen der Südkontinente. Die antarktischen Beziehungen. Zoogeographica 2, 246-304.

Woelkerling, W.J. 1975. On the epibiotic and pelagic Chlorophyceae, Phaeophyceae, and Rhodophyceae of the Western Sargasso Sea. Rhodora 77, 1-40.

Wong, C.S., Green, D.R. \& Cretney, W.J. 1974. Quantitative tar and plastic waste distributions in the Pacific Ocean. Nature 247, 30-32.

Woodcock, A.E. 1950. Subsurface pelagic Sargassum. Journal of Marine Research 9, 77-92.

Worcester, S.E. 1994. Adult rafting versus larval swimming — dispersal and recruitment of a botryllid ascidian on eelgrass. Marine Biology 121, 309-317.

Wurl, O. \& Obbard, J.P. 2004. A review of pollutants in the sea-surface microlayer (SML): a unique habitat for marine organisms. Marine Pollution Bulletin 48, 1016-1030.

Ye, S. \& Andrady, A.L. 1991. Fouling of floating plastic debris under Biscayne Bay exposure conditions. Marine Pollution Bulletin 22, 608-613.

Yeatman, H.C. 1962. The problem of dispersal of marine littoral copepods in the Atlantic Ocean, including some redescriptions of species. Crustaceana 4, 253-272.

Yerbury, J.W. 1919. Seashore diptera. Journal of the Marine Biological Association of the United Kingdom 12, 141-145.

Zaitsev, Y.P. 1970. Marine Neustonology. Jerusalem: Israel Program for Scientific Translations, 1971.

Zann, L.P. \& Harker, B.M. 1978. Egg production of the barnacles Platylepas ophiophilus Lanchester, Platylepas hexastylos (O. Fabricius), Octolasmis warwickii Gray and Lepas anatifera Linnaeus. Crustaceana 35, 206-213.

Zarate-Villafranco, A. \& Ortega-García, S. 2000. Spatial and seasonal distribution of the tuna catch on floating objects in the eastern Pacific Ocean during 1992-1993. Marine and Freshwater Behaviour and Physiology 34, 53-72. 